\title{
Estimação de Modelos Geoestatísticos com Dados Funcionais usando Ondaletas
}

\author{
Gilberto Pereira Sassi
}

\author{
TESE APRESENTADA \\ AO \\ Instituto DE MATEMÁTICA E EstATÍSTICA \\ DA \\ UNIVERSIDADE DE SÃO PAULO \\ PARA \\ OBTENÇÃO DO TÍTULO \\ $\mathrm{DE}$ \\ DOUTOR EM CIÊNCIAS
}

\section{Programa: Programa de Pós-graduação em Estatística Orientador: Profa. Dra. Chang Chiann}

Durante o desenvolvimento deste trabalho o autor recebeu auxílio financeiro da CAPES e CNPq

São Paulo, 03 de março de 2016 


\section{Estimação de Modelos Geoestatísticos com Dados Funcionais usando Ondaletas}

Esta versão da tese contém as correções e alterações sugeridas pela Comissão Julgadora durante a defesa da versão original do trabalho, realizada em 03/03/2016. Uma cópia da versão original está disponível no Instituto de Matemática e Estatística da Universidade de São Paulo.

Comissão Julgadora:

- Prof ${ }^{\mathrm{a}}$. Dr ${ }^{\mathrm{a}}$. Chang Chian (orientadora) - IME-USP

- Prof. Dr. Pedro Alberto Morettin - IME-USP

- Prof. Dr. João Ricardo Sato - CMCC-UFABC

- Prof. Dr. Luiz Koodi Hotta - IMECC-UNICAMP

- Prof ${ }^{a}$. Dr ${ }^{\mathrm{a}}$. Sílvia Regina Costa Lopes - IM-UFRGS 


\section{Agradecimentos}

Agradeço a minha família e meus amigos pelo suporte emocional nessa longa jornada de obter o título de doutor e a Profa. Dra. Chang Chiann pela orientação e pela paciência. Gostaria também de agradecer aos colegas de mestrado e doutorado Ariadne Nogueira, Bruno dos Santos, Daniel dos Reis e Joelson Campos por valiosas dicas de programação e redação. Agradeço em especial ao Joelson Campos que em momentos de dificuldade, cedeu o seu computador pessoal para realizar parte dos estudos de simulação contidos neste trabalho. 


\section{Resumo}

\section{SASSI, G.P. Estimação de Modelos Geoestatísticos com Dados Funcionais usando Ondaletas.}

2016. 142 f. Tese - Instituto de Matemática e Estatística, Universidade de São Paulo, São Paulo, 2016.

Com o recente avanço do poder computacional, a amostragem de curvas indexadas espacialmente tem crescido principalmente em dados ecológicos, atmosféricos e ambientais, o que conduziu a adpatação de métodos geoestatísticos para o contexto de Análise de Dados Funcionais. O objetivo deste trabalho é estudar métodos de krigagem para Dados Funcionais, adaptando os métodos de interpolação espacial em Geoestatística. Mais precisamente, em um conjunto de dados funcionais pontualmente fracamente estacionário e isotrópico, desejamos estimar uma curva em um ponto não monitorado no espaço buscando estimadores não viciados com erro quadrático médio mínimo. Apresentamos três abordagens para aproximar uma curva em sítio não monitorado, demonstramos resultados que simplificam o problema de otimização postulado pela busca de estimadores ótimos não viciados, implementamos os modelos em MATLAB usando ondaletas, que é mais adequada para captar comportamentos localizados, e comparamos os três modelos através de estudos de simulação. Ilustramos os métodos através de dois conjuntos de dados reais: um conjunto de dados de temperatura média diária das províncias marítimas do Canadá (New Brunswick, Nova Scotia e Prince Edward Island) coletados em 82 estações no ano 2000 e um conjunto de dados da CETESB (Companhia Ambiental do Estado de São Paulo) referentes ao índice de qualidade de ar MP10 em 22 estações meteorológicas na região metropolitana da cidade de São Paulo coletados no ano de 2014.

Palavras-chave: krigagem, geoestatística, ondaletas, análise de dados funcionais, estatísica espacial, MATLAB. 


\section{Abstract}

SASSI, G.P. Estimation of Geostatistical Models with Functional Data using Wavelets. 2016. 142 f. Tese - Instituto de Matemática e Estatística, Universidade de São Paulo, São Paulo, 2016.

The advance of the computational power in last decades has been generating a considerable increase in datasets of spatially indexed curves, mainly in ecological, atmospheric and environmental data, what have leaded to adjustments of geostatistes for the context of Functional Data Analysis. The goal of this work is to adapt the kriging methods from geostatistcs analysis to the framework of Functional Data Analysis. More precisely, we shall interpolate a curve in an unvisited spot searching for an unbiased estimator with minimum mean square error for a pointwise weakly stationary and isotropic functional dataset. We introduce three different approaches to estimate a curve in an unvisited spot, we demonstrate some results simplifying the optimization problem postulated by the optimality from these estimators, we implement the three models in MATLAB using wavelets and we compare them by simulation. We illustrate the ideas using two dataset: a real climatic dataset from Canadian maritime provinces (New Brunswick, Nova Scotia and Prince Edward Island) sampled at year 2000 in 82 weather station consisting of daily mean temperature and data from CETESB (environmental agency from the state of São Paulo, Brazil) sampled at 22 weather station in the metropolitan region of São Paulo city at year 2014 consisting of the air quality index PM10.

Keywords: kriging, geostatistcs, wavelets, functional data analysis, spatial statistics, MATLAB. 


\section{Sumário}

1 Introdução $\quad 1$

2 Conceitos Básicos $\quad 5$

2.1 Dados Funcionais . . . . . . . . . . . . . . . . . . . . . 5

2.1.1 Variável Funcional, Dado Funcional e Conjunto de Dados Funcional . . . . 5

2.2 Geoestatística . . . . . . . . . . . . . . . . . . . . 7

2.3 Ondaletas . . . . . . . . . . . . . . . . . . . . . 12

2.3.1 Função Escala e Função Ondaleta . . . . . . . . . . . . . . . . . . . . . . 13

2.3.2 Análise de Multirresolução . . . . . . . . . . . . . . . . . . . 16

3 Métodos de Krigagem para Dados Funcionais $\quad 21$

3.1 Krigagem Ordinária Funcional . . . . . . . . . . . . . . . . . . . . . . 22

3.2 Krigagem Tempo-Variante Funcional . . . . . . . . . . . . . . . . . . . 27

3.3 Krigagem Funcional por Campo . . . . . . . . . . . . . . . 36

4 Estudo de Simulação $\quad 41$

4.1 Medidas de Qualidade de Ajuste . . . . . . . . . . . . . . . . . . . . . 43

4.1 .1 Erro Quadrático Médio . . . . . . . . . . . . . . . . . . 43

4.1.2 Erro Quadrático Médio por Amostra . . . . . . . . . . . . . . . . . 44

4.2 Resultado - Simulação . . . . . . . . . . . . . . . . . . . . . 45

4.2.1 Simulação 1: Média Contínua . . . . . . . . . . . . . . . . 46

4.2.2 Simulação 2: Média Não Contínua . . . . . . . . . . . . . . . 50

5 Aplicação $\quad \mathbf{5 5}$

5.1 Temperatura Média Diária das Províncias Marítimas Canadenses . . . . . . . . . 56

5.2 Material Particulado Inalável - MP10 . . . . . . . . . . . . . . . . . 59

$\begin{array}{llr}6 & \text { Estudos Futuros } & 63\end{array}$

A Documentação da Toolbox krigingFDA do MATLAB $\quad 67$

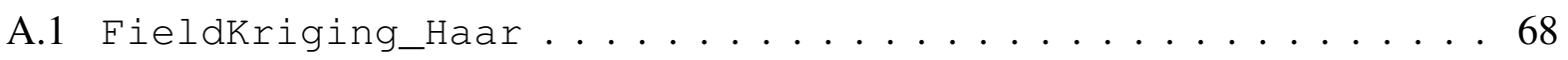

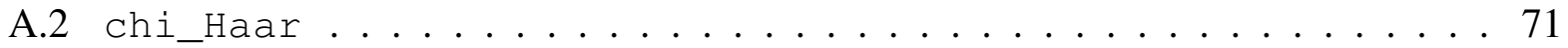

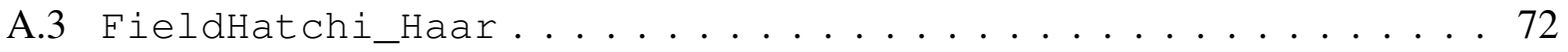


A.4 FieldKriging_dbN . . . . . . . . . . . . . . 73

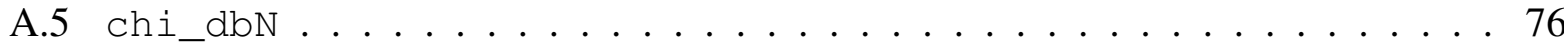

A.6 FieldHatchi_dbN . . . . . . . . . . . . . . . 79

A.7 Functionalkriging . . . . . . . . . . . . . . . 80

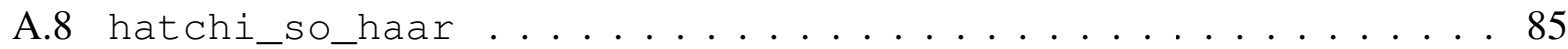

A.9 PointwiseFuncKriging . . . . . . . . . . . . 86

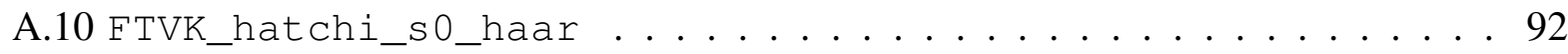

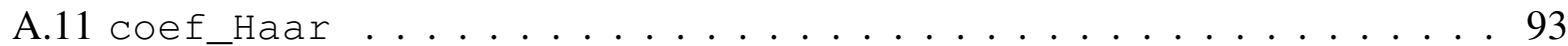

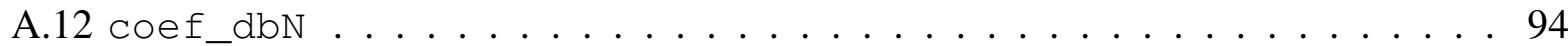

A.13 FuncKriging_dbN . . . . . . . . . . . . . . . 98

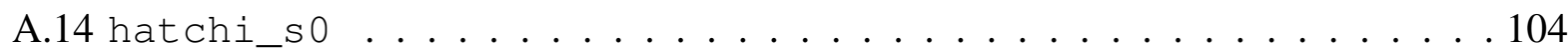

A.15 PointwiseFuncKriging_dbN . . . . . . . . . . . . 108

A.16 hatchi . . . . . . . . . . . . . . . . . . . 119

B Estações Meteorológicas - Províncias Marítimas Canadenses 121

C Estações Meteorológicas - Região Metropolitana de São Paulo 125

$\begin{array}{ll}\text { Referências Bibliográficas } & 127\end{array}$ 


\section{Capítulo 1}

\section{Introdução}

Um dos pilares da evolução e da sobrevivência dos seres humanos é a capacidade de realizar predições baseadas nas informações disponíveis. Por exemplo, no ato de atravessarmos uma rua, observamos o movimento e sabemos o momento correto de atravessar, pois temos um banco de informações que nos foi passado desde a infância de como responder a essas situações; economistas diariamente fazem predições da economia considerando informações sobre a situação da atividade econômica passada e presente; o painel intergovernamental sobre mudanças climáticas da Organização das Nações Unidas (IPCC-UN) realiza estudos sobre a temperatura global no médio e longo prazo; antes de decidir se deve distribuir uma revista em uma nova banca, uma editora calcula a previsão de vendas do novo ponto de venda usando pesquisas de demanda e a previsão de vendas nas bancas próximas. Ou seja, tanto na vida cotidiana quanto na ciência, a habilidade de previsões é imprescindível.

Note que em diversos campos do conhecimento, as informações disponíveis são georeferenciadas, isto é, sabemos as coordenadas (latitude e longitude) de cada observação, e desejamos prever o valor dessa variável em um ponto não monitorado. Este tipo de estudo é chamado de análise geoestatística e seu uso é empregado em áreas como: dados hidrológicos (Hevesi et al., 1992), mineração (Goovaerts, 1997; Journel e Huijbregts, 1978), estudos de qualidade do ar (Jerrett et al., 2005). Vide Ecker (2003) para uma revisão histórica, metodológica e exemplos de aplicação de Geoestatística.

Em Geoestaística, merecem destaque dois cientistas pioneiros: o engenheiro de minas sulafricano Danie Gerhardus Krige (Minnitt e Assibey-Bonsu, 2014) que propôs um método de pre- 
dição ótima com o objetivo de encontrar áreas com concentração de ouro (Krige, 1951) na década de 1950 e o matemático francês Georges François Paul Marie Matheron que formalizou o método proposto por Krige, chamando este método de predição ótima de kriging (krigagem em português), e fundou a Geoestatística com o seu trabalho (Matheron, 1963).

Com o avanço tecnológico e científico nas últimas décadas, as informações disponíveis não se restringem mais a valores escalares como no início do século XX. Imagens de satélite, curva de crescimento, curva de consumo, curva suavizada de temperatura medida a cada dez minutos são exemplos que deveriam ser tratados como curvas ao invés de valores escalares. Neste contexto, Ramsay e Dalzell (1991) introduziu o conceito de Análise de Dados Funcionais e desde então o seu uso se disseminou com aplicações em Modelos Lineares, Teoria de Resposta ao Item, Dados Dependentes, Análise Multivariada e outros (vide Ramsay e Silverman, 2002, para exemplos e aplicações de Análise de Dados Funcionais). Como referência para Análise de Dados Funcionais podemos citar: Ramsay (2006), Horváth e Kokoszka (2012) e Ferraty e Vieu (2006). Implementações de modelos para Dados Funcionais em MATLAB e em R estão documentadas em Ramsay et al. (2009).

Análogo ao caso escalar, existem aplicações, principalmente dados envolvendo ciências da terra e ambientais, em que as curvas no contexto de Dados Funcionais são espacialmente indexadas. Henao et al. (2010) e Henao et al. (2011) foram pioneiros em propor métodos de krigagem para Dados Funcionais. Salazar et al. (2015) apresentaram um preditor de krigagem para Dados Funcionais em que as curvas são funções densidade de probabilidade. Delicado et al. (2010) fazem uma revisão das técnicas de Geoestatística no contexto de Dados Funcionais. Bohorquez et al. (2015) propuseram uma metodologia e critérios de planejamento para encontrar o conjunto de localizações espaciais que minimiza a variância da predição ótima (krigagem para curvas) em um ponto não monitorado. Reyes et al. (2015) estenderam os métodos de krigagem em Dados Funcionais para a situação em que a função média de duas curvas não é necessariamente a mesma para duas localidades distintas. Nerini et al. (2010) propôs um método de interpolação espacial de curvas em que os parâmetros são funções definidas em um subconjunto de $\mathbb{R}^{2}$. A mesma abordagem foi apresentada na tese de doutorado de Henao (2009). Ignaccolo et al. (2014) introduziram um método de krigagem para Dados Funcionais adaptando regression kriging (vide Hengl et al., 2007, para uma revisão sobre regression kriging em Geoestatística), em que variáveis exógenas, 
potencialmente observadas como curvas, são permitidas. Caballero et al. (2013) forneceram uma solução para a krigagem para Dados Funcionais quando a condição de estacionaridade (verifique em Henao et al., 2011, as condições de estacionaridade) não é satisfeita adaptando o modelo de krigagem universal (vide Stein e Corsten, 1991, para uma revisão de krigagem universal em Geoestatística) para Dados Funcionais.

De uma forma geral, as abordagens de Geoestatística para Dados Funcionais usam splines e estimativas paramétricas para matriz de covariância cruzada. Neste contexto, propomos três abordagens de krigagem para Dados Funcionais usando ondaletas e matriz de semivariograma: exibimos uma implementação usando ondaletas para a Krigagem Ordinária Funcional proposto por Henao et al. (2011); propomos o uso do semivariograma com um estimador não paramétrico ao invés da matriz de covariância cruzada para a Krigagem Tempo-Variante Funcional apresentado por Henao et al. (2010) usando ondaletas; e, finalmente, expomos um novo método usando os coeficientes das projeções das curvas da amostra funcional nos espaços de aproximação na Análise de Multirresolução em Ondaletas. Convém notar que uso de ondaletas é mais adequado para captar comportamentos localizados; o semivariograma, que pode ser estimado não-parametricamente, é a medida de variabilidade usada em Geoestatística para métodos de krigagem e os métodos de interpolação espacial introduzidos neste trabalho apresentam erro quadrático médio menor que os modelos presentes na literatura, conforme estudo de simulação apresentado no Capítulo 4. Além disso, implementamos todos os métodos em MATLAB.

Organizamos esse texto como segue: no Capítulo 1 fazemos uma introdução; no Capítulo 2 revisamos alguns conceitos necessários para a compreensão dos métodos de krigagem para Dados Funcionais apresentados no Capítulo 3; no Capítulo 4 comparamos os três métodos explicitados no Capítulo 3 via simulação; no Capítulo 5 ilustramos esses modelos usando duas aplicações: dados de temperatura média no ano de 2000 em 82 estações meteorológicas localizadas nas províncias marítimas canadenses (Nova Scotia, New Brunswick e Prince Edward Island) e dados do índice MP10 ${ }^{1}$ da CETESB (Companhia Ambiental do Estado de São Paulo) no ano de 2014 em 22 estações meteorológicas na região metropolitana de São Paulo.

\footnotetext{
${ }^{1}$ MP10 é a quantidade em microgramas $(\mu g)$ de partículas inaláveis com diâmetro inferior a 10 micrômetros $(\mu m)$ em um $m^{3}$.
} 


\section{Capítulo 2}

\section{Conceitos Básicos}

Neste capítulo, descrevemos brevemente os conceitos fundamentais de dados funcionais, geoestatística e análise de ondaletas. Não pretendemos fazer um explanação profunda, apenas uma revisão sucinta de cada tópico e, para leitores interessados, fornecemos referências para cada assunto. Este capítulo está organizado em três seções: na Seção 2.1 apresentamos dados funcionais; na Seção 2.2 resumimos os conceitos principais de geoestatística; e na Seção 2.3 revisamos os conceitos fundamentais de análise de ondaleta.

\subsection{Dados Funcionais}

Nesta seção, apresentamos brevemente os conceitos referentes à análise de dados funcionais. Para um estudo mais profundo deste tópico há vários livros na literatura: Ramsay (2006), Ramsay e Silverman (2002), Ramsay et al. (2009), Horváth e Kokoszka (2012),

Ferraty e Vieu (2006), de Souza e Dias (2010) entre outros. Neste trabalho, seguiremos as definições e notações de Ferraty e Vieu (2006).

\subsubsection{Variável Funcional, Dado Funcional e Conjunto de Dados Funcional}

O progresso de ferramentas computacionais, em termos de processamento e armazenamento, faz-nos defrontar com banco de dados gigantesco. Por exemplo, é usual uma variável ser observada em vários pontos no intervalo $\left(t_{\min }, t_{\max }\right)$ e, então, uma observação pode ser expressa como uma família aleatória $\left\{X\left(t_{j}\right)\right\}_{j=1, \ldots, J}$. Quando a distância entre $t_{j}$ e $t_{j+1}$ é suficientemente pequena po- 
demos considerar a variável como proveniente de uma família contínua $\chi=\left\{X(t) \mid t \in\left(t_{\min }, t_{\max }\right)\right\}$. Além disso, há dados em que a natureza do estudo é nitidamente uma função contínua mesmo que a observação seja esparsa como, por exemplo, curva de crescimento e curva de consumo de energia elétrica ${ }^{1}$. Neste contexto, introduzimos os conceitos de variável funcional, dado funcional e conjunto de dados funcional.

Definição 2.1.1 (Variável Funcional). Uma variável aleatória $\chi=\{\chi(t) \mid t \in \mathbb{R}\}$ é chamada de variável funcional se sua imagem estiver contida em

$$
L^{2}(T)=\left\{f:\left.T \longrightarrow \mathbb{R}\left|\int_{0}^{1}\right| f(t)\right|^{2} d t\right\}
$$

com $T \subset \mathbb{R}^{n}$. Uma particular realização $\chi=\{\chi(t) \mid t \in \mathbb{R}\}$ de uma variável funcional $\chi$ é chamada de dado funcional.

Observações:

1. Quando $n=1$, chamamos a variável funcional $\chi=\{\chi(t) \mid t \in \mathbb{R}\}$ de uma curva aleatória e o dado funcional $\chi=\{\chi(t) \mid t \in \mathbb{R}\}$ de uma curva.

2. É importante ressaltar que existe a possibilidade de considerar uma superfície aleatória se $T \subset \mathbb{R}^{n}, n \geq 2$.

3. Neste texto, usaremos a notação $\chi(t)$ para denotar uma variável funcional e $\chi(t)$ para denotar um dado funcional proveniente de $\chi(t)$.

Com o conceito de variável e dado funcional, podemos definir o conceito de conjunto de dados funcional.

Definição 2.1.2 (Conjunto de Dados Funcional). Um conjunto de dados funcional ou amostra funcional $\chi_{1}(t), \ldots, \chi_{n}(t)$ são observações de $n$ variáveis funcionais $\chi_{1}(t), \ldots, \chi_{n}(t)$ identicamente distribuídas.

\footnotetext{
${ }^{1}$ Ramsay e Silverman (2002) apresenta uma modelagem completa dos dados de curva de crescimento, uma aplicação de dados funcionais em teoria de resposta ao item e muitos outros exemplos.
} 


\subsection{Geoestatística}

Nesta seção, apresentamos algumas definições básicas de geoestatística. Há vários clássicos de estatística espacial que podemos citar como referência: Diggle e Ribeiro (2007), Chiles e Delfiner (2009), Stein (2012), Haining (2003), Banerjee et al. (2014), Cressie e Cassie (1993) entre outros. Nesta seção, usamos as notações e definições conforme Banerjee et al. (2014).

Definição 2.2.1. Em um processo espacial $\boldsymbol{Y}(\boldsymbol{s})$, dizemos que $\boldsymbol{Y}\left(\boldsymbol{s}_{1}\right), \ldots, \boldsymbol{Y}\left(\boldsymbol{s}_{n}\right)$ são dados geoestatísticos se $\boldsymbol{Y}(\boldsymbol{s})$ é um vetor aleatório em uma localidade $s \in \mathbb{R}^{r}(r=2,3)$, em que $s$ varia continuamente em $D$ subconjunto de $\mathbb{R}^{r}$ que contém um subconjunto mensurável com medida positiva.

A seguir mostramos como calcular a distância entre dois pontos na superfície da terra. Para maiores detalhes, vide Frederick Pearson (1990).

\section{Calculando a distância na superfície da Terra}

Para duas localidades próximas, calcular a distância euclidiana pode ser uma excelente aproximação. Entretanto, para pontos distantes, a curvatura da Terra é relevante e precisa ser levada em conta no cálculo da distância.

Afirmação 2.2.1. Seja $P_{1}=\left(\theta_{1}, \lambda_{1}\right)$ e $P_{2}=\left(\theta_{2}, \lambda_{2}\right)$ dois pontos na superfície da Terra em que $\theta_{i}, i=1,2$ representa a latitude e $\lambda_{i}, i=1,2$ representa a longitude . A distância entre $P_{1}$ e $P_{2}$ é dada por

$$
\left\|P_{1}-P_{2}\right\|=R \cdot \phi
$$

em que $R$ é o raio da terra e $\phi$ satisfaz

$$
\cos \phi=\sin \theta_{1} \sin \theta_{2}+\cos \theta_{1} \cos \theta_{2} \cos \left(\lambda_{1}-\lambda_{2}\right),
$$

Demonstração. A geodésica em uma esfera é o arco do maior círculo passando por $P_{1}$ e $P_{2}$, ou seja, o comprimento de arco do círculo com raio R e passando por $P_{1}$ e $P_{2}$. Os pontos $P_{1}$ e $P_{2}$ em 
coordenadas cartesianas são obtidos por $u_{1}=\left(x_{1}, y_{1}, z_{1}\right)$ e $u_{2}=\left(x_{2}, y_{2}, z_{2}\right)$ com

$$
\left\{\begin{array} { l } 
{ x _ { 1 } = R \operatorname { c o s } \theta _ { 1 } \operatorname { c o s } \lambda _ { 1 } } \\
{ y _ { 1 } = R \operatorname { c o s } \theta _ { 1 } \operatorname { s i n } \lambda _ { 1 } } \\
{ z _ { 1 } = R \operatorname { s i n } \theta _ { 1 } }
\end{array} \quad \text { e } \quad \left\{\begin{array}{l}
x_{2}=R \cos \theta_{2} \cos \lambda_{2} \\
y_{2}=R \cos \theta_{2} \sin \lambda_{2} \\
z_{2}=R \sin \theta_{2}
\end{array}\right.\right.
$$

Note que $\left\|u_{1}\right\|=\left\|u_{2}\right\|=R$,

e

$$
\begin{aligned}
\left\|u _ { 1 } \left|\left\|\mid u_{2}\right\| \cos \phi\right.\right. & =\left\langle u_{1} ; u_{2}\right\rangle \\
& =x_{1} x_{2}+y_{1} y_{2}+z_{1} z_{2} \\
& =R^{2} \cos \theta_{1} \cos \theta_{2}\left(\cos \lambda_{1} \cos \lambda_{2}+\sin \lambda_{1} \sin \lambda_{2}\right)+R^{2} \sin \theta_{1} \theta_{2} \\
& =R^{2} \cos \theta_{1} \cos \theta_{2} \cos \left(\lambda_{1}-\lambda_{2}\right)+R^{2} \sin \theta_{1} \sin \theta_{2} .
\end{aligned}
$$

Assim, o ângulo entre $P_{1}$ e $P_{2}$ é a solução da equação (2.1)

$$
\cos \phi=\cos \theta_{1} \cos \theta_{2} \cos \left(\lambda_{1}-\lambda_{2}\right)+\sin \theta_{1} \sin \theta_{2}
$$

e a distância entre $P_{1}$ e $P_{2}$ é $R \phi$.

Observação. Para calcular a distância $R \phi$ entre $P_{1}$ e $P_{2}$ usamos a Mapping Toolbox do MATLAB.

Ponderando que podemos calcular numericamente a distância entre dois pontos na superfície da terra, considere o processo estocástico $\{Y(s) \mid s \in D\}, D \subseteq \mathbb{R}^{r}$. Quando $r=1$, há uma extensa literatura em séries temporais analisando este tipo de processo estocástico. Quando $r>1$, dizemos que o processo estocástico é um processo espacial e existe um interesse especial quando $r=2$ (Norte-Sul e leste-oeste) e quando $r=3$ (Norte-sul, leste-oeste e nível acima do mar). Na prática, os dados serão uma realização parcial desse processo espacial em um conjunto finito de pontos $s_{1}, \ldots, s_{n}$ e desejamos predizer $Y(s)$ em $s \notin\left\{s_{1}, \ldots, s_{n}\right\}$.

Antes de introduzir os conceitos fundamentais de estacionaridade e isotropia para um processo espacial em geoestatística, estabelecemos as seguintes notações:

i. $\mu(s)=\mathrm{E}(Y(s)), s \in D$; 
ii. O processo espacial é Gaussiano se para quaisquer $n$ pontos $\boldsymbol{s}_{1}, \ldots, \boldsymbol{s}_{n}$ contidos em $D$, temos que $Y\left(\boldsymbol{s}_{1}\right), \ldots, Y\left(\boldsymbol{s}_{n}\right)$ tem um distribuição normal multivariada de ordem $\mathrm{n}$.

Definição 2.2.2 (Estacionaridade). Considere o processo espacial $\{Y(s) \mid s \in D\}$ com média $\mu(s), \forall s \in D$. Então dizemos que o processo espacial é

a. Estritamente estacionário se para quaisquer $\boldsymbol{s}_{1}, \ldots, \boldsymbol{s}_{n}$ com $\boldsymbol{s}_{i} \in D, i=1, \ldots, n$, e $\boldsymbol{h} \in$ $\mathbb{R}^{r}$ com $\boldsymbol{s}_{i}+\boldsymbol{h} \in D, i=1, \ldots, n$, a distribuição conjunta de $Y\left(\boldsymbol{s}_{1}\right), \ldots, Y\left(\boldsymbol{s}_{n}\right)$ é a mesma de $Y\left(\boldsymbol{s}_{1}+\boldsymbol{h}\right), \ldots, Y\left(\boldsymbol{s}_{n}+\boldsymbol{h}\right)$.

\section{b. Fracamente estacionário se}

i) a média é constante, ou seja, $\mu(s)=\mu, \forall s \in D$;

ii) a covariância depende apenas do vetor de separação $\boldsymbol{h}$, ou seja, $\operatorname{Cov}(Y(\boldsymbol{s}) ; Y(\boldsymbol{s}+\boldsymbol{h}))=$ $C(\boldsymbol{h}), \forall \boldsymbol{s} \in D, \forall \boldsymbol{h} \in \mathbb{R}^{r} \operatorname{com} \boldsymbol{s}+\boldsymbol{h} \in D$.

\section{c. Intrinsecamente estacionário se}

i) $\mathrm{E}(Y(\boldsymbol{s}+\boldsymbol{h})-Y(\boldsymbol{s}))=0, \forall \boldsymbol{s} \in D, \forall \boldsymbol{h} \in \mathbb{R}^{r} \operatorname{com} \boldsymbol{s}+\boldsymbol{h} \in D$;

ii) $\mathrm{E}(Y(\boldsymbol{s}+\boldsymbol{h})-Y(\boldsymbol{s}))^{2}=\operatorname{Var}(Y(\boldsymbol{s}+\boldsymbol{h})-Y(\boldsymbol{s}))=2 \gamma(\boldsymbol{h}), \forall \boldsymbol{s} \in D, \forall \boldsymbol{h} \in \mathbb{R}^{r}$ com $\boldsymbol{s}+\boldsymbol{h} \in D$.

Observações:

1. Estacionaridade fraca implica que a covariância do processo espacial entre duas localidades quaisquer pode ser sumarizada sem prejuízo por uma função $C(\boldsymbol{h})$ que depende apenas do vetor de separação $\boldsymbol{h}$.

2. Se um processo espacial é fracamente estacionário e Gaussiano, então ele é estritamente estacionário.

3. Na literatura de processo espacial, $2 \gamma(\boldsymbol{h})$ é chamado de variograma, $\gamma(\boldsymbol{h})$ é chamado de semivariograma e $C(\boldsymbol{h})$ é chamado de covariograma.

4. Estacionaridade intrínseca é definida em termos dos dois primeiros momentos de $Y(\boldsymbol{s}+\boldsymbol{h})-$ $Y(\boldsymbol{s})$. Nenhuma suposição é feita em termos da distribuição conjunta de $Y\left(\boldsymbol{s}_{1}\right), \ldots, Y\left(\boldsymbol{s}_{n}\right)$. 
5. Conhecendo o covariograma podemos obter o variograma, de fato

$$
\begin{aligned}
2 \gamma(\boldsymbol{h}) & =\operatorname{Var}(Y(\boldsymbol{s}+\boldsymbol{h})-Y(\boldsymbol{s})) \\
& =\operatorname{Var}(Y(\boldsymbol{s}+\boldsymbol{h}))-2 \operatorname{Cov}(Y(\boldsymbol{s}+\boldsymbol{h}) ; Y(\boldsymbol{s}))+\operatorname{Var}(Y(\boldsymbol{s})) \\
& =2 C(\mathbf{0})-2 C(\boldsymbol{h}),
\end{aligned}
$$

ou seja, $\gamma(\boldsymbol{h})=C(\mathbf{0})-C(\boldsymbol{h})$.

6. Estacionaridade fraca implica estacionaridade intrínseca, mas a recíproca não é verdade.

7. Para um processo intrinsecamente estacionário, o semivariograma $\gamma(h)$ é definido não positivo condicional, isto é, se $\boldsymbol{s}_{1}, \ldots, \boldsymbol{s}_{n} \in D$ e $a_{1}, \ldots, a_{n} \in \mathbb{R} \operatorname{com} \sum_{i=1}^{n} a_{i}=0$, então $\sum_{i=1}^{n} \sum_{j=1}^{n} a_{i} a_{j} \gamma\left(\boldsymbol{s}_{i}-\boldsymbol{s}_{j}\right) \leq 0$.

De fato,

$$
\begin{aligned}
\sum_{i=1}^{n} \sum_{j=1}^{n} a_{i} a_{j} \gamma\left(\boldsymbol{s}_{i}-\boldsymbol{s}_{j}\right) & =\frac{1}{2} \sum_{i=1}^{n} \sum_{j=1}^{n} a_{i} a_{j} \mathrm{E}\left[\left(Y\left(\boldsymbol{s}_{i}\right)-Y\left(\boldsymbol{s}_{j}\right)\right)^{2}\right] \\
& =\frac{1}{2} \sum_{i=1}^{n} \sum_{j=1}^{n} a_{i} a_{j} \mathrm{E}\left[\left(Y\left(\boldsymbol{s}_{i}\right)^{2}\right)\right]-\sum_{i=1}^{n} \sum_{j=1}^{n} a_{i} a_{j} \mathrm{E}\left(Y\left(\boldsymbol{s}_{i}\right) Y\left(\boldsymbol{s}_{j}\right)\right)+ \\
& +\frac{1}{2} \sum_{i=1}^{n} \sum_{j=1}^{n} a_{i} a_{j} \mathrm{E}\left[\left(Y\left(\boldsymbol{s}_{j}\right)\right)^{2}\right] \\
& =-\mathrm{E}\left[\sum_{i=1}^{n} \sum_{j=1}^{n} a_{i} a_{j} Y\left(\boldsymbol{s}_{i}\right) Y\left(\boldsymbol{s}_{j}\right)\right] \\
& =-\mathrm{E}\left[\left(\sum_{i=1}^{n} a_{i} Y\left(\boldsymbol{s}_{i}\right)\right)^{2}\right] \\
& \leq 0 .
\end{aligned}
$$

Para analisar o caso em que estacionaridade intrínseca implica estacionaridade fraca introduzimos o conceito de ergodicidade para processos espaciais.

Definição 2.2.3 (Ergodicidade). Dizemos que um processo espacial é ergódico se $\operatorname{Cov}(Y(\boldsymbol{s}+\boldsymbol{h}) ; Y(\boldsymbol{s})) \longrightarrow 0$ quando $\|\boldsymbol{h}\| \longrightarrow \infty$.

Em outras palavras, a Definição 2.2.3 estabelece que em um processo espacial ergódico o covari- 
ograma tende a zero quando os pontos ficam distantes em $D \subseteq \mathbb{R}^{r}$. Com essa suposição podemos estabelecer condições em que um processo espacial intrinsecamente estacionário é fracamente estacionário conforme o Teorema 2.2.2 a seguir. Além disso, este teorema estabelece uma técnica para calcular o covariograma usando o semivariograma.

Teorema 2.2.2. Seja $\{Y(s) \mid s \in D\}$ um processo espacial intrinsecamente estacionário e ergódico com variância constante e suponha que $\lim _{\|\boldsymbol{h}\| \longrightarrow \infty} \gamma(\boldsymbol{h})$ existe. Então, o covariograma é dado por

$$
C(\boldsymbol{h})=\lim _{\|\boldsymbol{u}\| \longrightarrow \infty} \gamma(\boldsymbol{u})-\gamma(\boldsymbol{h})
$$

Demonstração. Em primeiro lugar, observe que $C(\boldsymbol{h})=C(\mathbf{0})-\gamma(\boldsymbol{h})$. Como o processo é ergódico temos que $\lim _{\|\boldsymbol{h}\| \longrightarrow \infty} C(\boldsymbol{h})=0$ e então $C(\mathbf{0})=\lim _{\|\boldsymbol{h}\| \longrightarrow \infty} \gamma(\boldsymbol{h})$, ou seja,

$$
C(\boldsymbol{h})=\lim _{\|\boldsymbol{h}\| \longrightarrow \infty} \gamma(\boldsymbol{h})-\gamma(\boldsymbol{h})
$$

Outra propriedade importante em processos espaciais é o conceito de isotropia que será definida a seguir.

Definição 2.2.4 (Isotropia). Considere um processo espacial com semivariograma $\gamma(\boldsymbol{h})$. Dizemos que o processo é isotrópico se

$$
\gamma\left(\boldsymbol{h}_{1}\right)=\gamma\left(\boldsymbol{h}_{2}\right), \quad \text { para }\left\|\boldsymbol{h}_{1}\right\|=\left\|\boldsymbol{h}_{2}\right\|
$$

Se o processo não é isotrópico, dizemos que ele é anisotrópico.

Se o processo é intrinsecamente estacionário e isotrópico dizemos que ele é homogêneo.

Observações:

1. Para um processo isotrópico, $\gamma(\boldsymbol{h})$ é uma função de $\|\boldsymbol{h}\|$ e usamos a notação $\gamma(\|\boldsymbol{h}\|)$. 


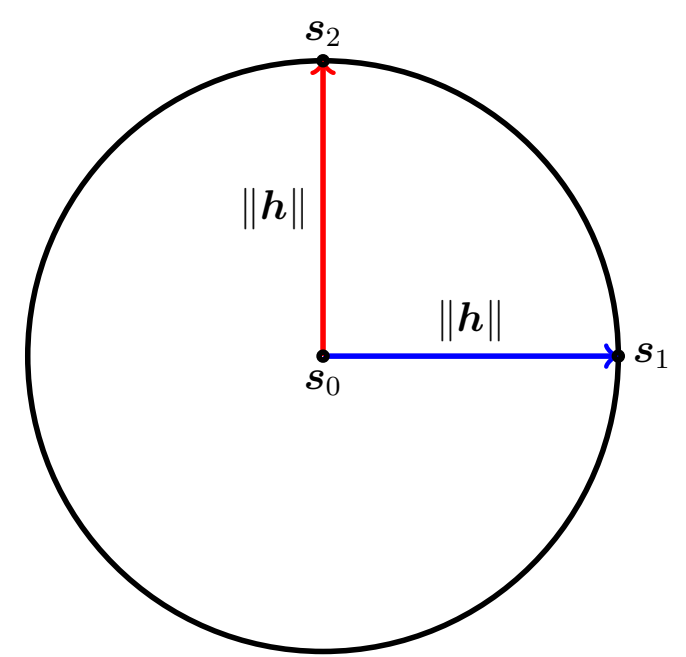

Figura 2.1: Ilustração do conceito de isotropia: o semivariograma entre $s_{0}$ e $s_{1}$ e o semivariograma entre $s_{0}$ e $s_{1}$ é o mesmo.

2. Processos espaciais isotrópicos são simples, facilmente interpretáveis e seus modelos paramétricos dependem de poucos parâmetros.

3. Para um processo espacial isotrópico, um estimador não-paramétrico para o semivariograma é o semivariograma empírico dado por

$$
\hat{\gamma}(h)=\frac{1}{2|N(h)|} \sum_{(i, j) \in N(h)}\left[Y\left(\boldsymbol{s}_{i}\right)-Y\left(\boldsymbol{s}_{j}\right)\right]^{2},
$$

em que $N(h)=\left\{(i, j) \in \mathbb{N} \times \mathbb{N} \mid\left\|s_{i}-\boldsymbol{s}_{j}\right\|=t\right\}$ e $|N(h)|$ é o número de pontos de $N(h)$.

\subsection{Ondaletas}

A ideia, tanto na análise de séries de Fourier, na aproximação de Taylor, na análise de ondaletas ou em qualquer outra base, é aproximar uma função por uma combinação de senos e cossenos, polinômios ou ondaletas. Particularmente no caso de ondaletas, desejamos aproximar uma função $f \in L^{2}(\mathbb{R})=\left\{f:\left.\mathbb{R} \rightarrow \mathbb{R}\left|\int_{-\infty}^{\infty}\right| f(u)\right|^{2} d u<\infty\right\}$ por uma combinação linear de dilatações binárias $2^{j}$ e translações diádicas $k 2^{-j}$ de funções $\phi(x)$, chamada de função escala ou ondaleta pai, e / ou de funções $\psi(x)$, chamada de função ondaleta ou ondaleta mãe.

O campo de aplicação de ondaletas é extenso e inclui processamento de sinais, tratamento de imagens, compressão de dados, estimação de densidade, regressão não paramétrica e outros. Como referência podemos citar Boggess e Narcowich (2009), Daubechies et al. (1992), Meyer e Ryan 
(1993), Morettin (2014), Meyer e Salinger (1995) e Chui (2014).

Nesta seção, expomos as ideias básicas sobre ondaletas, começando pela Seção 2.3.1 onde descrevemos a função ondaleta, também chamada de ondaleta mãe, e a função escala, também denominada ondaleta pai, e na Seção 2.3.2 onde apresentamos o conceito de análise de multirresolução.

\subsubsection{Função Escala e Função Ondaleta}

Considere o espaço $L^{2}(\mathbb{R})=\left\{f:\left.\mathbb{R} \rightarrow \mathbb{R}\left|\int_{\mathbb{R}}\right| f(u)\right|^{2} d u<\infty\right\}$ das funções quadrado integráveis em $\mathbb{R}$. A ideia é considerar dilatações (ou compressões) e translações de uma função $\psi$ de modo a cobrir todo $\mathbb{R}$. Mais precisamente, consideramos

$$
\psi_{j, k}(t)=2^{\frac{j}{2}} \psi\left(2^{j} t-k\right)
$$

em que $\left\{\psi_{j, k}(t) \mid j, k \in \mathbb{Z}\right\}$ é uma base para $L^{2}(\mathbb{R})$ e $\psi$ é chamada de ondaleta mãe ou função ondaleta.

Suponha que $\left\{\psi_{j, k}(t) \mid j, k \in \mathbb{Z}\right\}$ é uma base ortonormal para $L^{2}(\mathbb{R})^{2}$, isto é,

$$
\left\langle\psi_{j, k}, \psi_{j, m}\right\rangle=\delta_{j, l} \delta_{k, m}, \quad j, k, l, m \in \mathbb{Z}
$$

em que $\delta_{i, j}=\left\{\begin{array}{l}1, \text { se } i=j \\ 0, \text { caso contrário }\end{array} \quad \mathrm{e}\right.$

$$
f(t)=\sum_{j=-\infty}^{\infty} \sum_{k=-\infty}^{\infty} d_{j, k} \psi_{j, k}(t)
$$

em que a convergência é considerada em média quadrática. Adicionalmente, a série na equação (2.2) é chamada de série de ondaletas de $f(t)$ e $d_{j, k}, j, k \in \mathbb{Z}$ são denominados coeficientes

\footnotetext{
${ }^{2}$ Meyer (1985) demonstrou que existe uma função $\psi(x)$ tal que $\left\{\psi_{j, k}(x) \mid j, k \in \mathbb{Z}\right\}$ é uma base ortonormal para $L^{2}(\mathbb{R})$.
} 
de ondaletas ou coeficientes de detalhes e são calculados por

$$
\begin{aligned}
d_{j, k} & =\left\langle f(t), \psi_{j, k}(t)\right\rangle \\
& =\int_{-\infty}^{\infty} f(u) \psi_{j, k}(u) d u
\end{aligned}
$$

Neste contexto de ortogonalidade e analogamente a análise de série de Fourier, a relação de parseval é válida. Mais precisamente,

$$
\int_{-\infty}^{\infty} f(u) d u=\sum_{j=-\infty}^{\infty} \sum_{k=-\infty}^{\infty} d_{j, k}^{2}
$$

Além disso, as seguintes propriedades para a ondaleta mãe são válidas:

i. $\int_{-\infty}^{\infty} \psi(t) d t=0$,

ii. $\int_{-\infty}^{\infty}|\psi(t)| d t<\infty$,

iii. $\int_{-\infty}^{\infty}|\psi(t)|^{2} d t=1$,

iv. Existe $r \in\{j \in \mathbb{Z} \mid j \geq 1\}$ tal que

$$
\begin{aligned}
& \int_{-\infty}^{\infty} t^{j} \psi(t) d t=0, \quad j=0,1, \ldots, r-1 \\
& \int_{-\infty}^{\infty}\left|t^{r} \psi(t)\right| d t<\infty
\end{aligned}
$$

O valor de $r$ na propriedade iv. está ligado ao grau de regularidade (suavidade) de $\psi$ : quanto maior o valor de $r$, mais suave será o $\psi$. Ademais, se a ondaleta tem suporte compacto, o valor de $r$ está relacionado ao seu suporte (vide Härdle et al., 1998, para maiores detalhes).

Poderíamos ter considerado translações (ou compressões) e dilatações de uma função $\phi$, denominada função escala ou ondaleta pai, para aproximar $f(t)$. A função escala está intrinsecamente relacionada com a ondaleta mãe através das equações de dilatação descritas pela equações (2.3) e 
(2.4).

$$
\begin{aligned}
\phi(t) & =\sqrt{2} \sum_{k} l_{k} \phi(2 t-k), \\
\psi(t) & =\sqrt{2} \sum_{k} h_{k} \phi(2 t-k) .
\end{aligned}
$$

em que $h_{k}$ e $l_{k}$ são associadas pela igualdade $h_{k}=(-1)^{k} l_{1-k}$, chamada de quadrature mirror filter relation. Além disso, temos que

$$
\begin{aligned}
l_{k} & =\sqrt{2} \int_{-\infty}^{\infty} \phi(t) \phi(2 t-k) d t, \\
h_{k} & =\sqrt{2} \int_{-\infty}^{\infty} \psi(t) \phi(2 t-k) d t,
\end{aligned}
$$

em que $l_{k}$ é denominado filtro passo-baixo e $h_{k}$ é chamado de filtro passo-alto.

Exemplo 2.3.1. O exemplo mais antigo e mais simples é o caso Haar, introduzido inicialmente por Alfred Haar no trabalho Haar (1909). A ideia consiste em aproximar funções quadrado integráveis por funções escadas.

A ondaleta Haar é descrita por

$$
\psi(t)= \begin{cases}1, & 0 \geq t<\frac{1}{2} \\ -1, & \frac{1}{2} \geq t<1 \\ 0, & \text { caso contrário }\end{cases}
$$

para qual a função escala é dada por $\phi(t)=\left\{\begin{array}{ll}1, & t \in[0,1) \\ 0, & \text { caso contário }\end{array}\right.$.

Note que $\psi(t)$ e $\phi(t)$ tem suporte compacto e

$$
\begin{aligned}
\phi(t) & =\phi(2 t)+\phi(2 t-1) \\
& =\frac{1}{\sqrt{2}} \sqrt{2} \phi(2 t)+\frac{1}{\sqrt{2}} \sqrt{2} \phi(2 t-1),
\end{aligned}
$$


e

$$
\begin{aligned}
\psi(t) & =\phi(2 t)-\phi(2 t-1) \\
& =\frac{1}{\sqrt{2}} \sqrt{2} \phi(2 t)-\frac{1}{\sqrt{2}} \sqrt{2} \phi(2 t-1),
\end{aligned}
$$

ou seja, $l_{0}=l_{1}=\sqrt{\frac{1}{2}}, h_{0}=l_{0}$ e $h_{1}=-l_{1}$. Ademais, é possível verificar que $\left\{\psi_{j, k}(t) \mid j, k \in \mathbb{Z}\right\}$ é uma base ortonormal para $L^{2}(\mathbb{R})$. Na Figura 2.2 mostramos o gráfico da função escala e na Figura 2.3 a ondaleta Haar.

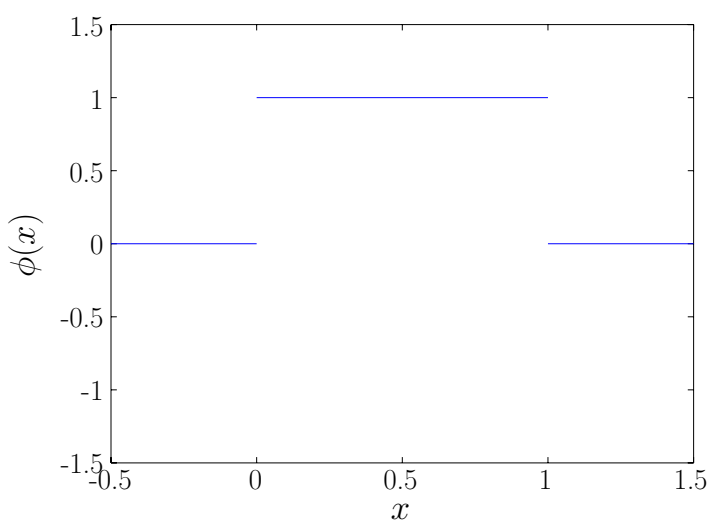

Figura 2.2: Função escala Haar.

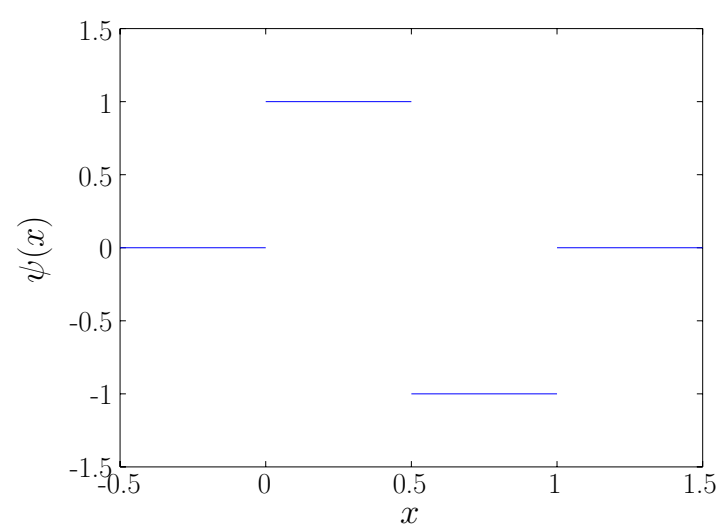

Figura 2.3: Ondaleta Haar.

Exemplo 2.3.2. Além das ondaletas Haar, as ondaletas Daubechies (daublets) são ondaletas ortogonais de suporte compacto bastante utilizadas. Essas ondaletas dependem de um parâmetro $N$ que controla a suavidade de $\phi$ e $\psi$ e são obtidas usando os filtros passa-baixo e passo-alto (vide Daubechies et al., 1992, para maiores detalhes). Como ilustração, na Figura 2.5 mostramos o gráfico da ondaleta mãe para $N=2,3,4,5$ e na Figura 2.4 apresentamos a função escala para $N=2,3,4,5$.

\subsubsection{Análise de Multirresolução}

De acordo com Morettin (2014), a análise de multirresolução permite analisar os dados disponíveis em várias escalas de resolução. Formalmente, temos que:

Definição 2.3.1. Uma coleção de subespaços vetoriais $\left\{V_{j} \mid j \in \mathbb{Z}\right\}$ de $L^{2}(\mathbb{R})$ é uma análise de multirresolução se as seguintes condições são satifeitas: 

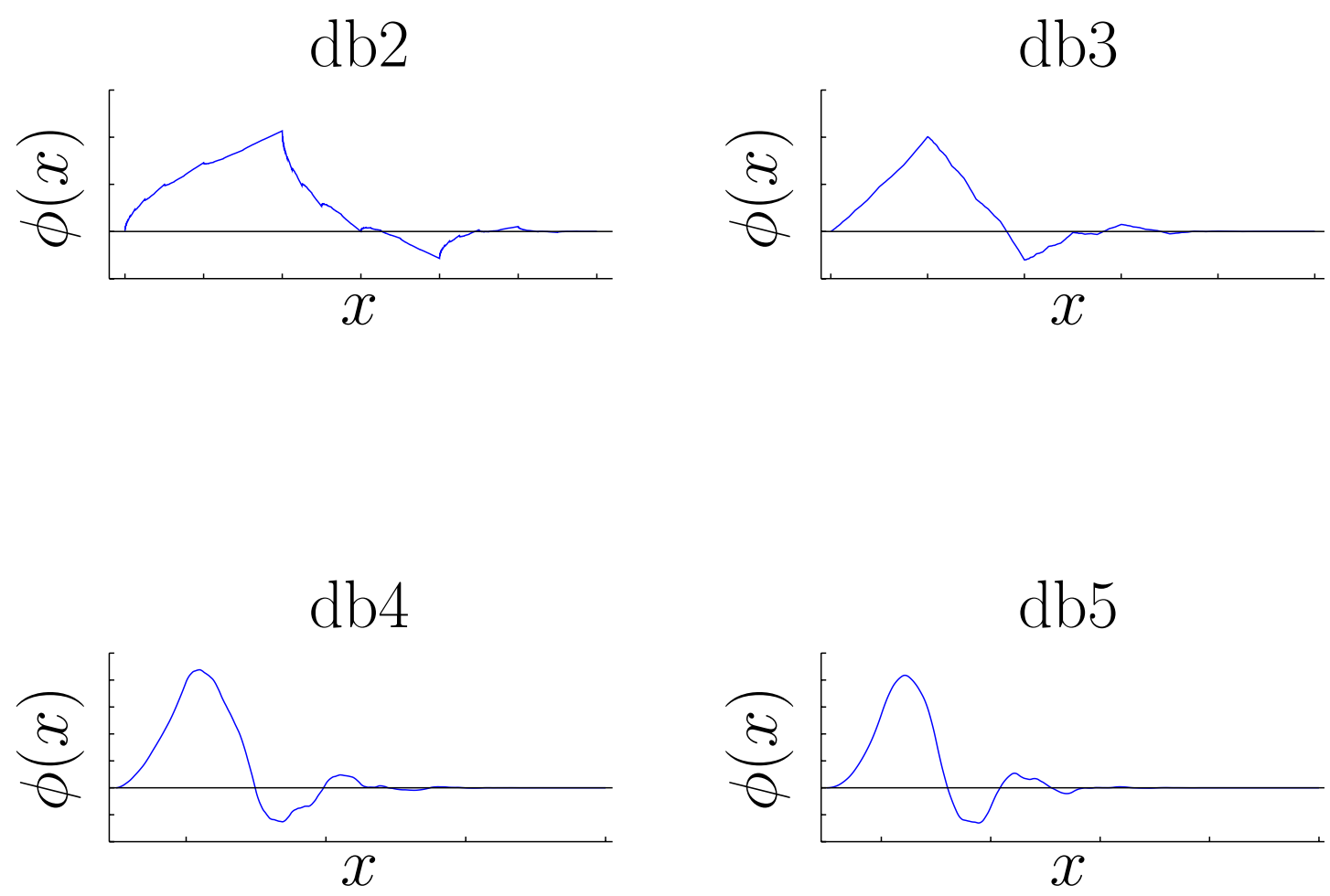

Figura 2.4: Função escala para a família Daubechies quando $N=2,3,4,5$.

(aninhamento) $V_{j} \subset V_{j+1}, \forall j \in \mathbb{Z}$;

(densidade) $L^{2}(\mathbb{R})=\overline{\bigcup_{j \in \mathbb{Z}} V_{j}}$;

(separação) $\bigcap_{j \in \mathbb{Z}} V_{j}=\{0\}$

(escolamento) $f(x) \in V_{j} \Leftrightarrow f(2 x) \in V_{j+1}, \forall j \in \mathbb{Z}$.

Denominamos $V_{j}$ de espaço de aproximação e $j$ de nível de aproximação ou nível de resolução.

Em uma análise de multirresolução $\left\{V_{j} \mid j \in \mathbb{Z}\right\}$, aproximamos uma função $f(t) \in L^{2}(\mathbb{R})$ ao nível de resolução $j$ pela projeção de $f(t)$ em $V_{j}$. A propriedade de aninhamento implica que quando passamos do nível $j$ ao $j+1$, ganhamos informação sobre $f(t)$ e no limite $(j \rightarrow \infty)$ recuperamos completamente $f(t)$. Por outro lado, quando passamos do nível $j$ ao $j-1$, perdemos informação sobre $f(t)$ e no limite $(j \rightarrow-\infty)$ a projeção é a função nula. 

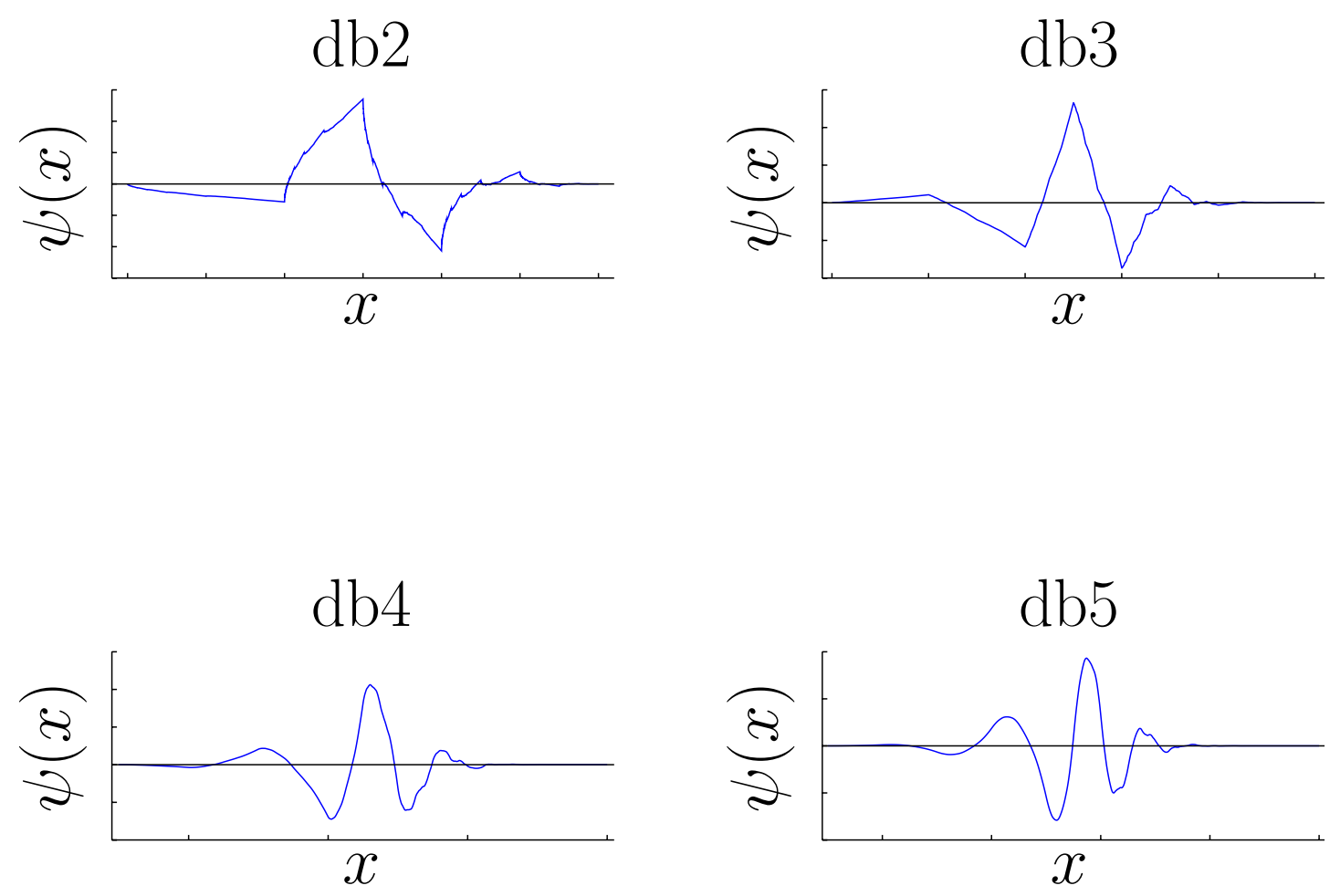

Figura 2.5: Ondaleta mãe para a família Daubechies quando $N=2,3,4,5$.

Pode-se provar que existe uma função $\phi \in L^{2}(\mathbb{R})$ tal que, $\left\{\phi_{j, k} \mid k \in \mathbb{Z}\right\}$ é uma base ortonormal de $V_{j}$ (Mallat, 1989a,b). Ademais, a informação perdida quando passamos de $V_{j+1}$ a $V_{j}$ pode ser representada pelo subespaço $W_{j}$, ou seja, $V_{j+1}=V_{j} \oplus W_{j}$ e, consequentemente, $V_{j}=\bigoplus_{k=-\infty}^{\infty} W_{k}$. Pode-se mostrar que $\left\{\psi_{j, k} \mid k \in \mathbb{Z}\right\}$ é uma base ortornormal para $W_{j}$. Observe também que,

$$
\begin{aligned}
L^{2}(\mathbb{R}) & =\bigoplus_{j=-\infty}^{\infty} W_{j} \\
& =V_{j} \oplus \bigoplus_{k=j}^{\infty} W_{k} \\
& =\frac{\bigcup_{j=-\infty}^{\infty} V_{j},}{}
\end{aligned}
$$

e, então, os conjuntos $\left\{\phi_{j, k} \mid j, k \in \mathbb{Z}\right\},\left\{\psi_{j, k} \mid j, k \in \mathbb{Z}\right\}$ e $\left\{\phi_{j, k} \mid k \in \mathbb{Z}\right\} \cup\left\{\psi_{l, k} \mid l, k \in\right.$ 
$\mathbb{Z} \operatorname{com} l \geq j\}$ são bases ortonormais para $L^{2}(\mathbb{R})$.

Nesse trabalho, optamos pelo uso das ondaletas Haar e Daubechies por terem suporte compacto e uma certa regularidade. Além disso, escolhemos aproximar uma curva através da projeção de $f(t) \in L^{2}(\mathbb{R})$ em $V_{j}$, isto é, fazemos uso apenas da função escala. No Capítulo 3, apresentamos as três abordagens de estimação de uma curva em um ponto não monitorado, em que usamos os conceitos apresentados neste capítulo. 


\section{Capítulo 3}

\section{Métodos de Krigagem para Dados}

\section{Funcionais}

Neste trabalho, estamos num contexto em que temos uma amostra funcional $\chi_{\boldsymbol{s}_{1}}(t), \ldots, \chi_{\boldsymbol{s}_{n}}(t)$, em que $\chi_{\boldsymbol{s}_{i}}(t), i=1, \ldots, n, t \in[0,1]$, é uma curva amostrada no ponto $\boldsymbol{s}_{i}=$ $\left(\theta_{i}, \eta_{i}\right)$ da região em estudo $\operatorname{com} \theta_{i}$ sendo a latitude e $\eta_{i}$ a longitude, e o objetivo é estimar a curva $\chi_{s_{0}}(t)$ em um ponto não monitorado $s_{0}=\left(\theta_{0}, \eta_{0}\right)$. Três abordagens diferentes de estimação são apresentadas: a primeira proposta é estimar $\chi_{s_{0}}(t)$ por meio de uma combinação linear das curvas $\chi_{\boldsymbol{s}_{i}}(t), i=1, \ldots, n$, conforme descrito na Seção 3.1; o segundo método de estimação propõe o uso de uma combinação linear em que os coeficientes são funções ao invés de constantes escalares conforme detalhado na 3.2; e, finalmente, na Seção 3.3 propomos um novo método de krigagem usando os coeficientes das expansões em ondaletas das curvas $\chi_{\boldsymbol{s}_{i}}(t), i=1, \ldots, n$. A inovação dos métodos implementados nesta tese consiste no uso de ondaletas, no lugar de B-splines, para as duas primeiras abordagens além de propor o uso dos coeficientes da aproximação de $\chi_{\boldsymbol{s}_{1}}(t), \ldots, \chi_{\boldsymbol{s}_{n}}(t)$ para obter uma estimativa para $\chi_{\boldsymbol{s}_{0}}(t)$.

Em todos os modelos de krigagem deste trabalho, supomos que o processo estocástico espacial associado à amostra funcional é pontualmente fracamente estacionário e isotrópico. Mais precisamente,

i. $\mathrm{E}\left[\boldsymbol{\chi}_{\boldsymbol{s}}(t)\right]=m(t), \forall \boldsymbol{s} \in D, \forall t \in[0,1]$;

ii. $\operatorname{Cov}\left(\chi_{\boldsymbol{s}_{1}}(t), \chi_{\boldsymbol{s}_{2}}(t)\right)=C\left(\left\|\boldsymbol{s}_{1}-\boldsymbol{s}_{2}\right\| ; t\right), \forall \boldsymbol{s}_{1}, \boldsymbol{s}_{2} \in D, \forall t \in[0,1]$ em que $\|\cdot\|$ é a geodésica 
entre dois pontos na superfície da Terra conforme descrito na Seção 2.2 (vide Banerjee et al., 2014, para maiores detalhes);

iii. $\frac{1}{2} \operatorname{Var}\left(\chi_{\boldsymbol{s}_{1}}(t)-\chi_{\boldsymbol{s}_{2}}(t)\right)=\gamma\left(\left\|\boldsymbol{s}_{1}-\boldsymbol{s}_{2}\right\| ; t\right), \forall \boldsymbol{s}_{1}, \boldsymbol{s}_{2} \in D, \forall t \in[0,1]$. Para $t \in[0,1]$ fixo, $\gamma\left(\left\|\boldsymbol{s}_{1}-\boldsymbol{s}_{2}\right\| ; t\right)$ é chamada de variograma.

\subsection{Krigagem Ordinária Funcional}

Esse método de interpolação espacial para curvas pode ser considerado o modelo mais simples abordado neste trabalho e foi introduzido inicialmente por Henao et al. (2011), onde eles usaram B-splines para aproximar as curvas $\chi_{\boldsymbol{s}_{1}}(t), \ldots, \chi_{\boldsymbol{s}_{n}}(t)$.

A estimativa $\hat{\chi}_{s_{0}}(t)$ da curva na localização $\boldsymbol{s}_{0}$ é combinação linear das curvas da amostra, isto é,

$$
\hat{\chi}_{\boldsymbol{s}_{0}}(t)=\sum_{i=1}^{n} \lambda_{i} \chi_{\boldsymbol{s}_{i}}(t)
$$

em que $\lambda_{1}, \ldots, \lambda_{n}$ são estimados por

$$
\arg \min _{\lambda_{1}, \ldots, \lambda_{n}} \mathrm{E}\left[\left\|\hat{\chi}_{\boldsymbol{s}_{0}}(t)-\chi_{\boldsymbol{s}_{0}}(t)\right\|^{2}\right] \text { sujeito a } \mathrm{E}\left[\hat{\chi}_{\boldsymbol{s}_{0}}(t)-\boldsymbol{\chi}_{\boldsymbol{s}_{0}}(t)\right]=0
$$

Em outras palavras, desejamos encontrar $\lambda_{1}, \ldots, \lambda_{n}$ tal que $\hat{\chi}_{s_{0}}(t)$ seja não viciado com erro quadrático médio mínimo. Note que resolver o problema de otimização não linear (3.2) pode ser uma tarefa analítica e computacionalmente complexa, mas Henao et al. (2011) apresentaram uma alternativa viável para resolver este problema apresentado no Teorema 3.1.1.

Teorema 3.1.1 (Henao et al. (2011)). Resolver o problema de otimização não linear

$$
\arg \min _{\lambda_{1}, \ldots, \lambda_{n}} \mathrm{E}\left[\left\|\hat{\chi}_{\boldsymbol{s}_{0}}(t)-\boldsymbol{\chi}_{\boldsymbol{s}_{0}}(t)\right\|^{2}\right] \text { sujeito } \text { a } \mathrm{E}\left[\hat{\chi}_{\boldsymbol{s}_{0}}(t)-\boldsymbol{\chi}_{\boldsymbol{s}_{0}}(t)\right]=0,
$$


é equivalente a resolver o sistema de equações lineares dado por

$$
\left(\begin{array}{ccccc}
\gamma\left(\left\|\boldsymbol{s}_{1}-\boldsymbol{s}_{1}\right\|\right) & \gamma\left(\left\|\boldsymbol{s}_{2}-\boldsymbol{s}_{1}\right\|\right) & \ldots & \gamma\left(\left\|\boldsymbol{s}_{n}-\boldsymbol{s}_{1}\right\|\right) & 1 \\
\gamma\left(\left\|\boldsymbol{s}_{1}-\boldsymbol{s}_{2}\right\|\right) & \gamma\left(\left\|\boldsymbol{s}_{2}-\boldsymbol{s}_{2}\right\|\right) & \ldots & \gamma\left(\left\|\boldsymbol{s}_{n}-\boldsymbol{s}_{2}\right\|\right) & 1 \\
\vdots & \vdots & \ddots & \vdots & \vdots \\
\gamma\left(\left\|\boldsymbol{s}_{1}-\boldsymbol{s}_{n}\right\|\right) & \gamma\left(\left\|\boldsymbol{s}_{2}-\boldsymbol{s}_{n}\right\|\right) & \ldots & \gamma\left(\left\|\boldsymbol{s}_{n}-\boldsymbol{s}_{n}\right\|\right) & 1 \\
1 & 1 & \ldots & 1 & 0
\end{array}\right) \cdot\left(\begin{array}{c}
\lambda_{1} \\
\vdots \\
\lambda_{n} \\
\mu
\end{array}\right)=\left(\begin{array}{c}
\gamma\left(\left\|\boldsymbol{s}_{1}-\boldsymbol{s}_{0}\right\|\right) \\
\vdots \\
\gamma\left(\left\|\boldsymbol{s}_{n}-\boldsymbol{s}_{0}\right\|\right) \\
1
\end{array}\right)
$$

em que $\gamma(h)=\int_{0}^{1} \gamma(h, t) d t$ é chamado de traço variograma.

Demonstração. Em primeiro lugar, note que

$$
\mathrm{E}\left[\boldsymbol{\chi}_{\boldsymbol{s}_{0}}(t)\right]=m(t), \forall s \in D, \forall t \in[0,1]
$$

e a restrição pode ser reescrita em

$$
\begin{aligned}
\mathrm{E}\left[\hat{\chi}_{\boldsymbol{s}_{0}}(t)\right] & =\mathrm{E}\left[\boldsymbol{\chi}_{\boldsymbol{s}_{0}}(t)\right] \\
& =m(t)
\end{aligned}
$$

Mas observe que

$$
\begin{aligned}
\mathrm{E}\left[\hat{\chi}_{\boldsymbol{s}_{0}}(t)\right] & =\sum_{i=1}^{n} \lambda_{i} \mathrm{E}\left[\boldsymbol{\chi}_{\boldsymbol{s}_{i}}(t)\right] \\
& =m(t) \sum_{i=1}^{n} \lambda_{i}
\end{aligned}
$$

e, consequentemente, a restrição $\mathrm{E}\left[\hat{\chi}_{\boldsymbol{s}_{0}}(t)-\chi_{\boldsymbol{s}_{0}}(t)\right]=0$ é equivalente a $\sum_{i=1}^{n} \lambda_{i}=1$.

Além disso, pelo Teorema de Fubini e sob a restrição de $\mathrm{E}\left[\hat{\chi}_{\boldsymbol{s}_{0}}(t)-\chi_{\boldsymbol{s}_{0}}(t)\right]=0$, temos que

$$
\begin{aligned}
\mathrm{E}\left[\left\|\hat{\chi}_{\boldsymbol{s}_{0}}(t)-\boldsymbol{\chi}_{\boldsymbol{s}_{0}}(t)\right\|^{2}\right] & =\mathrm{E}\left[\int_{0}^{1}\left|\hat{\chi}_{\boldsymbol{s}_{0}}(t)-\chi_{\boldsymbol{s}_{0}}(t)\right|^{2} d t\right] \\
& =\int_{0}^{1} \operatorname{Var}\left(\hat{\chi}_{\boldsymbol{s}_{0}}(t)-\boldsymbol{\chi}_{\boldsymbol{s}_{0}}(t)\right) d t
\end{aligned}
$$

Como $\chi_{\boldsymbol{s}_{s}}(t), s \in D$ é um processo estocástico pontualmente fracamente estacionário, $\sum_{i=1}^{n} \lambda_{i}=$ 
1 e $C(h ; t)=C(0 ; t)-\gamma(h ; t)$, temos que,

$$
\begin{aligned}
\operatorname{Var}\left(\hat{\chi}_{\boldsymbol{s}_{0}}(t)-\chi_{\boldsymbol{s}_{0}}(t)\right) & =\operatorname{Var}\left(\chi_{\boldsymbol{s}_{0}}(t)\right)-2 \sum_{i=1}^{n} \lambda_{i} \operatorname{Cov}\left(\chi_{\boldsymbol{s}_{i}}(t) ; \chi_{\boldsymbol{s}_{0}}(t)\right)+\operatorname{Var}\left(\sum_{i=1}^{n} \lambda_{i} \chi_{\boldsymbol{s}_{i}}\right) \\
& =C(0 ; t)-2 \sum_{i=1}^{n} \lambda_{i}\left[C(0 ; t)-\gamma\left(\left\|\boldsymbol{s}_{i}-\boldsymbol{s}_{0}\right\|\right)\right]+\sum_{i=1}^{n} \lambda_{i}^{2} C(0 ; t)+ \\
& +2 \sum_{j=1}^{n} \sum_{k=j+1}^{n} \lambda_{j} \lambda_{k}\left[C(0 ; t)-\gamma\left(\left\|\boldsymbol{s}_{j}-\boldsymbol{s}_{k}\right\| ; t\right)\right] \\
& =C(0 ; t)-2 C(0 ; t) \sum_{i=1}^{n} \lambda_{i}+C(0 ; t)\left[\sum_{i=1}^{n} \lambda_{i}^{2}+2 \sum_{j=1}^{n} \sum_{k=j+1}^{n} \lambda_{j} \lambda_{k}\right]+ \\
& +2 \sum_{i=1}^{n} \lambda_{i} \gamma\left(\left\|\boldsymbol{s}_{i}-\boldsymbol{s}_{0}\right\| ; t\right)-2 \sum_{j=1}^{n} \sum_{k=j+1}^{n} \lambda_{j} \lambda_{k} \gamma\left(\left\|\boldsymbol{s}_{j}-\boldsymbol{s}_{k}\right\| ; t\right)-\sum_{i=1}^{n} \lambda_{i}^{2} \gamma(0 ; t) \\
& =2 \sum_{i=1}^{n} \lambda_{i} \gamma\left(\left\|\boldsymbol{s}_{i}-\boldsymbol{s}_{0}\right\| ; t\right)-2 \sum_{j=1}^{n} \sum_{k=j+1}^{n} \lambda_{j} \lambda_{k} \gamma\left(\left\|\boldsymbol{s}_{j}-\boldsymbol{s}_{k}\right\| ; t\right)-\sum_{i=1}^{n} \lambda_{i}^{2} \gamma(0 ; t),
\end{aligned}
$$

$\operatorname{com} \gamma(0 ; t)=0$.

Logo, temos que,

$$
\begin{aligned}
\mathrm{E}\left[\left\|\hat{\chi}_{\boldsymbol{s}_{0}}(t)-\chi_{\boldsymbol{s}_{0}}(t)\right\|^{2}\right] & =\int_{0}^{1} \operatorname{Var}\left(\hat{\chi}_{\boldsymbol{s}_{0}}(t)-\chi_{\boldsymbol{s}_{0}}(t)\right) d t \\
& =2 \sum_{i=1}^{n} \lambda_{i} \gamma\left(\left\|\boldsymbol{s}_{i}-\boldsymbol{s}_{0}\right\|\right)-2 \sum_{j=1}^{n} \sum_{k=j+1}^{n} \lambda_{j} \lambda_{k} \gamma\left(\left\|\boldsymbol{s}_{j}-\boldsymbol{s}_{k}\right\|\right)-\sum_{i=1}^{n} \lambda_{i}^{2} \gamma(0),
\end{aligned}
$$

em que $\gamma(h ; t)=\int_{0}^{1} \gamma(h ; t) d t$ é chamado de traço variograma.

Em outras palavras, desejamos resolver o seguinte problema de otimização linear

$$
\begin{aligned}
& \arg \min _{\lambda_{1}, \ldots, \lambda_{n}} 2 \sum_{i=1}^{n} \lambda_{i} \gamma\left(\left\|\boldsymbol{s}_{i}-\boldsymbol{s}_{0}\right\|\right)-2 \sum_{j=1}^{n} \sum_{k=j+1}^{n} \lambda_{j} \lambda_{k} \gamma\left(\left\|\boldsymbol{s}_{j}-\boldsymbol{s}_{k}\right\|\right)-\sum_{i=1}^{n} \lambda_{i}^{2} \gamma(0) \\
& \text { sujeito a } \sum_{i=1}^{n} \lambda_{i}=1
\end{aligned}
$$

que pode ser resolvido usando multiplicadores de Lagrange. Mais precisamente, sejam

$$
\begin{aligned}
& f\left(\lambda_{1}, \ldots, \lambda_{n}\right)=2 \sum_{i=1}^{n} \lambda_{i} \gamma\left(\left\|\boldsymbol{s}_{i}-\boldsymbol{s}_{0}\right\|\right)-2 \sum_{j=1}^{n} \sum_{k=j+1}^{n} \lambda_{j} \lambda_{k} \gamma\left(\left\|\boldsymbol{s}_{j}-\boldsymbol{s}_{k}\right\|\right)-\sum_{i=1}^{n} \lambda_{i}^{2} \gamma(0) \\
& g\left(\lambda_{1}, \ldots, \lambda_{n}\right)=\sum_{i=1}^{n} \lambda_{i} .
\end{aligned}
$$


Então a encontrar a solução do problema de otimização linear (3.4) é equivalente a resolver o sistema de equações lineares dado por

$$
\left\{\begin{array}{l}
\nabla f\left(\lambda_{1}, \ldots, \lambda_{n}\right)=\delta \nabla g\left(\lambda_{1}, \ldots, \lambda_{n}\right), \\
g\left(\lambda_{1}, \ldots, \lambda_{n}\right)=1
\end{array}\right.
$$

isto é,

$$
\left\{\begin{array}{l}
2 \gamma\left(\left\|\boldsymbol{s}_{i}-\boldsymbol{s}_{0}\right\|\right)-2 \sum_{j=1}^{n} \lambda_{j} \gamma\left(\left\|\boldsymbol{s}_{j}-\boldsymbol{s}_{i}\right\|\right)=\delta, i=1, \ldots, n \\
\sum_{i=1}^{n} \lambda_{i}=1
\end{array}\right.
$$

Em forma matricial, temos que

$$
\left(\begin{array}{ccccc}
\gamma\left(\left\|s_{1}-s_{1}\right\|\right) & \gamma\left(\left\|s_{2}-s_{1}\right\|\right) & \ldots & \gamma\left(\left\|s_{n}-s_{1}\right\|\right) & 1 \\
\gamma\left(\left\|s_{1}-s_{2}\right\|\right) & \gamma\left(\left\|s_{2}-s_{2}\right\|\right) & \ldots & \gamma\left(\left\|s_{n}-s_{2}\right\|\right) & 1 \\
\vdots & \vdots & \ddots & \vdots & \vdots \\
\gamma\left(\left\|s_{1}-s_{n}\right\|\right) & \gamma\left(\left\|s_{2}-s_{n}\right\|\right) & \ldots & \gamma\left(\left\|s_{n}-s_{n}\right\|\right) & 1 \\
1 & 1 & \ldots & 1 & 0
\end{array}\right) \cdot\left(\begin{array}{c}
\lambda_{1} \\
\vdots \\
\lambda_{n} \\
\mu
\end{array}\right)=\left(\begin{array}{c}
\gamma\left(\left\|s_{1}-s_{0}\right\|\right) \\
\vdots \\
\gamma\left(\left\|s_{n}-s_{0}\right\|\right) \\
1
\end{array}\right)
$$

em que $\mu=-\frac{\delta}{2}$

Para construir o sistema linear do Teorema 3.1.1, precisamos de um estimador para o traço variograma e Henao et al. (2011) propuseram o seguinte estimador não paramétrico

$$
\hat{\gamma}(h)=\frac{1}{2|N(h)|} \sum_{(i, j) \in N(h)} \int_{0}^{1}\left(\chi_{\boldsymbol{s}_{i}}(t)-\chi_{\boldsymbol{s}_{j}}(t)\right)^{2} d t
$$

em que $N(h)=\left\{(i, j) \in\{1, \ldots, n\} \times\{1, \ldots, n\} \mid\left\|s_{i}-s_{j}\right\| \in(h-\epsilon, h+\epsilon)\right\}$ para $\epsilon>0$ apropriado e $|N(h)|$ é o número de elementos de $N(h)$. Na Figura 3.1, ilustramos o conjunto $N(h)$.

Na prática, não observamos a curva inteira, apenas uma série temporal $\chi_{\boldsymbol{s}_{i}}\left(\frac{k-1}{2^{L}}\right), k=$ $1, \ldots, 2^{L}$. Para estimar cada curva $\chi_{s_{i}}(t), t \in[0,1]$, usamos regressão não-paramétrica ( vide Härdle et al., 1998; Ogden, 1997; Vidakovic, 2009, para maiores detalhes sobre regressão não 


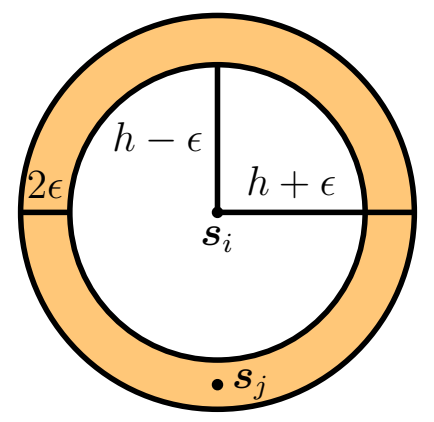

Figura 3.1: Gráfico ilustrativo de $N(h)$ com $\epsilon>0$. Note que $(i, j) \in N(h)$, pois $h-\epsilon<\left\|s_{i}-s_{j}\right\|<h+\epsilon$.

paramétrica usando ondaletas ). Mais precisamente, para $s_{i}$, observamos $Y_{j}=\chi_{\boldsymbol{s}_{i}}\left(\frac{j-1}{2^{L}}\right), j=$ $1, \ldots, 2^{L}$ e desejamos realizar a regressão descrita pela equação (3.5),

$$
Y_{j}=f_{\boldsymbol{s}_{i}}\left(x_{j}\right)+\epsilon_{j}, \quad j=1, \ldots, 2^{L}
$$

em que $x_{j}=\frac{j-1}{2^{L}}, j=1, \ldots, 2^{L}$. A função estimada $\hat{f}_{\boldsymbol{s}_{i}}(x), x \in[0,1]$ pela equação (3.5) será a nossa curva de interesse, ou seja, $\hat{\chi}_{s_{i}}(t)=\hat{f}_{\boldsymbol{s}_{i}}(t), t \in[0,1]$.

Ao nível de precisão $J=\frac{L}{2}$, a função estimada $\hat{f}_{\boldsymbol{s}_{i}}(\cdot)$ é a projeção de $f_{\boldsymbol{s}_{i}}(\cdot)$ em $V_{J}$ dada por

$$
\begin{aligned}
\hat{f}_{\boldsymbol{s}_{i}}(u) & =\left(P^{J} f_{\boldsymbol{s}_{i}}\right)(u) \\
& =\sum_{k \in \mathbb{Z}} c_{i, k}^{J} \phi_{J, k}(u),
\end{aligned}
$$

em que $P^{J} f_{\boldsymbol{s}_{i}}$ é a projeção ortogonal de $f_{\boldsymbol{s}_{i}}$ em $V_{J}$. Como escolhemos trabalhar com ondaletas de suporte compacto e $\chi_{\boldsymbol{s}_{i}}(t), i=0,1, \ldots, n$, tem também suporte compacto, existe $M \in \mathbb{N}$ tal que $c_{i, k}^{J}=0$ para $|k|>M$.

Em forma matricial, temos que

$$
\chi_{\boldsymbol{s}_{i}}(t) \approx \boldsymbol{c}_{i}^{\top} \phi_{J}(t)
$$

com $\boldsymbol{c}_{i}^{\top}=\left(c_{i,-M}^{J}, \ldots, c_{i, M}^{J}\right)^{\top}$ e $\phi^{\top}(t)=\left(\phi_{J,-M}(t), \ldots, \phi_{J, M}(t)\right)^{\top}$. Pela propriedade da ortonormalidade 
da base de ondaletas, o estimador não paramétrico para o traço variograma se resume a

$$
\begin{aligned}
\hat{\gamma}(h) & \approx \frac{1}{2|N(h)|} \sum_{(j, k) \in N(h)} \int_{0}^{1}\left(\boldsymbol{c}_{j}-\boldsymbol{c}_{k}\right)^{\top} \boldsymbol{\phi}_{J}(t) \boldsymbol{\phi}_{J}^{\top}(t)\left(\boldsymbol{c}_{j}-\boldsymbol{c}_{k}\right) d t \\
& =\frac{1}{2|N(h)|} \sum_{(j, k) \in N(h)}\left(\boldsymbol{c}_{j}-\boldsymbol{c}_{k}\right)^{\top}\left(\boldsymbol{c}_{j}-\boldsymbol{c}_{k}\right) .
\end{aligned}
$$

\subsection{Krigagem Tempo-Variante Funcional}

Este modelo de interpolação espacial denominado Krigagem Tempo-Variante Funcional pode ser considerado uma melhoria do método apresentado na Seção 3.1 e foi proposto por Henao et al. (2010), em que B-splines foram utilizadas para aproximar as curvas $\chi_{\boldsymbol{s}_{i}}(t), i=1, \ldots, n$. A idéia é estimar $\chi_{\boldsymbol{s}_{0}}(t)$ como uma combinação linear das curvas $\chi_{\boldsymbol{s}_{i}}(t), i=1, \ldots, n$, mas em vez de considerar constantes $\lambda_{1}, \ldots, \lambda_{n}$, desejamos estimar funções $\lambda_{1}(t), \ldots, \lambda_{n}(t)$. Mais precisamente,

$$
\hat{\chi}_{\boldsymbol{s}_{0}}(t)=\sum_{i=1}^{n} \lambda_{i}(t) \chi_{\boldsymbol{s}_{i}}(t)
$$

em que aproximamos as funções $\lambda_{i}(t), i=1, \ldots, n$, usando ondaletas. Então, temos que $\lambda_{i}(t) \approx$ $\boldsymbol{b}_{i}^{\top} \boldsymbol{\phi}_{J}(t), i=1, \ldots, n$ e $\chi_{\boldsymbol{s}_{i}}(t) \approx \boldsymbol{a}_{i}^{\top} \boldsymbol{\phi}_{J}(t), i=0,1, \ldots, n, \mathrm{com}$

$$
\begin{aligned}
\phi_{J}(t) & =\left(\phi_{J,-M}(t), \ldots, \phi_{J, M}(t)\right)^{\top}, \\
\boldsymbol{a}_{i} & =\left(a_{i,-M}^{J}, \ldots, a_{i, M}^{J}\right)^{\top}, i=0,1, \ldots, n, \\
\boldsymbol{b}_{i} & =\left(b_{i,-M}^{J}, \ldots, b_{i, M}^{J}\right)^{\top}, i=1, \ldots, n,
\end{aligned}
$$

e, então, desejamos estimar o seguinte problema de otimização não linear:

$$
\boldsymbol{b}_{1}, \ldots, \boldsymbol{b}_{n}=\arg \min _{\boldsymbol{b}_{1}, \ldots, \boldsymbol{b}_{n}} \mathrm{E}\left[\left\|\hat{\chi}_{\boldsymbol{s}_{0}}(t)-\boldsymbol{\chi}_{\boldsymbol{s}_{0}}(t)\right\|^{2}\right] \text { sujeito a } \mathrm{E}\left[\hat{\chi}_{\boldsymbol{s}_{0}}(t)-\boldsymbol{\chi}_{\boldsymbol{s}_{0}}(t)\right]=0
$$

Note que resolver o problema de otimização linear descrito pela Equação (3.6) pode não ser uma tarefa simples. O Teorema 3.2.1 fornece uma simplificação do problema de otimização não linear (3.6).

Teorema 3.2.1. Suponha que $\boldsymbol{a}_{0}, \boldsymbol{a}_{1} \ldots, \boldsymbol{a}_{n}$ são observações de um campo aleatório multivariado 
fracamente estacionário e isotrópico, então resolver o problema de otimização não linear dado por

$$
\boldsymbol{b}_{1}, \ldots, \boldsymbol{b}_{n}=\arg \min _{\boldsymbol{b}_{1}, \ldots, \boldsymbol{b}_{n}} \mathrm{E}\left[\left\|\hat{\chi}_{\boldsymbol{s}_{0}}(t)-\chi_{\boldsymbol{s}_{0}}(t)\right\|^{2}\right] \quad \text { sujeito } a \mathrm{E}\left[\hat{\chi}_{\boldsymbol{s}_{0}}(t)-\chi_{\boldsymbol{s}_{0}}(t)\right]=0
$$

é equivalente a encontrar o ponto de mínimo da forma quadrática descrita por

$$
\boldsymbol{\beta}^{\top} Q \boldsymbol{\beta}-2 \boldsymbol{\beta}^{\top} \boldsymbol{P}
$$

em que

$$
\begin{aligned}
\boldsymbol{\beta} & =\left(\boldsymbol{b}_{1}^{\top}, \ldots, \boldsymbol{b}_{n}^{\top}, \boldsymbol{m}^{\top}\right)^{\top} \\
Q & =\left(\begin{array}{ccccc}
Q_{11} & Q_{12} & \ldots & Q_{1 n} & I_{N} \\
Q_{21} & Q_{22} & \ldots & Q_{2 n} & I_{N} \\
\vdots & \vdots & \ddots & \vdots & \vdots \\
Q_{n 1} & Q_{n 2} & \ldots & Q_{n n} & I_{N} \\
I_{N} & I_{N} & \ldots & I_{N} & \mathbf{0}_{N}
\end{array}\right) \\
P & =\left(\boldsymbol{P}_{1}^{\top}, \ldots, \boldsymbol{P}_{n}^{\top}, \boldsymbol{c}^{\top}\right)^{\top}
\end{aligned}
$$

com

$$
\begin{aligned}
Q_{i, j} & =\int_{0}^{1} \boldsymbol{\phi}_{J}(t) \phi_{J}(t)^{\top} \operatorname{Cov}\left(\boldsymbol{a}_{i} ; \boldsymbol{a}_{j}\right) \boldsymbol{\phi}_{J}(t) \boldsymbol{\phi}_{J}(t)^{\top} d t \\
\boldsymbol{P}_{i} & =\int_{0}^{1} \boldsymbol{\phi}_{J}(t) \boldsymbol{\phi}_{J}(t)^{\top} \operatorname{Cov}\left(\boldsymbol{a}_{i} ; \boldsymbol{a}_{0}\right) \boldsymbol{\phi}_{J}(t) d t \\
\boldsymbol{c}^{\top} \boldsymbol{\phi}_{J}(t) & \approx \begin{cases}1, & \text { se } t \in[0,1] \\
0, & \text { caso contrário }\end{cases}
\end{aligned}
$$

onde $N$ é ordem da matriz $Q_{11}, \mathbf{0}_{N}$ é matriz nula de ordem $N$ e $I_{N}$ é a matriz identidade de ordem $N$.

Demonstração. Antes de prosseguir, observamos que a demonstração desse teorema é uma adaptação para ondaletas dos resultados demonstrados por Henao et al. (2010) para BSplines. 
Primeiramente, observe que a restrição $\mathrm{E}\left[\hat{\chi}_{\boldsymbol{s}_{0}}(t)-\chi_{\boldsymbol{s}_{0}}(t)\right]=0$ é equivalente a $\sum_{i=1}^{n} \lambda_{i}(t)=$ $1, \forall t \in[0,1]$, pois $\chi_{\boldsymbol{s}}(t), s \in D$ é pontualmente fracamente estacionário. Além disso, pelo Teorema de Fubini, temos que

$$
\mathrm{E}\left[\left\|\hat{\chi}_{\boldsymbol{s}_{0}}(t)-\chi_{\boldsymbol{s}_{0}}(t)\right\|^{2}\right]=\int_{0}^{1} \operatorname{Var}\left(\hat{\chi}_{\boldsymbol{s}_{0}}(t)-\chi_{\boldsymbol{s}_{0}}(t)\right) d t
$$

Note que usamos as aproximações

$$
\begin{aligned}
\lambda_{i}(t) & \approx \boldsymbol{b}_{i}^{\top} \boldsymbol{\phi}_{J}(t), \\
\chi_{\boldsymbol{s}_{i}}(t) & \approx \boldsymbol{a}_{i}^{\top} \boldsymbol{\phi}_{J}(t), \\
\chi_{\boldsymbol{s}_{0}}(t) & \approx \boldsymbol{a}_{0}^{\top} \boldsymbol{\phi}_{J}(t),
\end{aligned}
$$

$\operatorname{com} \sum_{i=1}^{n} \lambda_{i}(t)=\sum_{i=1}^{n} \boldsymbol{b}_{i} \boldsymbol{\phi}_{J}(t)=1, \forall t \in[0,1]$, isto é,

$$
\sum_{i=1}^{n} \sum_{k=-M}^{M} b_{i, k}^{J} \phi_{J, k}(t)=1, \forall t \in[0,1]
$$

Note que podemos aproximar a função constante 1 por $\boldsymbol{\alpha}^{\top} \phi_{J}(t) \approx 1 \operatorname{com} \boldsymbol{\alpha}=\left(\alpha_{-M}, \ldots, \alpha_{M}\right)^{\top}$ e, então, temos que

$$
\sum_{i=1}^{n} \sum_{k=-M}^{M} b_{i, k} \phi_{J, k}(t)=\sum_{i=1}^{n} \alpha_{k} \phi_{J, k}(t)
$$

e, consequentemente, temos que

$$
\left\langle\sum_{i=1}^{n} \sum_{k=-M}^{M} b_{i, k}^{J} \phi_{J, k}(t) ; \phi_{J, l}(t)\right\rangle=\left\langle\sum_{i=1}^{n} \alpha_{k} \phi_{J, k}(t) ; \phi_{J, l}(t)\right\rangle, \quad l=-M, \ldots, M
$$

ou seja,

$$
\sum_{i=1}^{n} b_{i, l}^{J}=\alpha_{l}, \quad l=-M, \ldots, M
$$


ou em forma matricial

$$
\left(\begin{array}{c}
\sum_{i=1}^{n} b_{i,-M}^{J} \\
\vdots \\
\sum_{i=1}^{n} b_{i, 0}^{J} \\
\vdots \\
\sum_{i=1}^{n} b_{i, M}^{J}
\end{array}\right)=\left(\begin{array}{c}
\alpha_{-M} \\
\vdots \\
\alpha_{0} \\
\vdots \\
\alpha_{M}
\end{array}\right)
$$

Ou seja, a restrição $\sum_{i=1}^{n} \lambda_{i}(t)=1$ pode ser reescrita por $\sum_{i=1}^{n} \boldsymbol{b}_{i}=\boldsymbol{\alpha}$.

Observe que $\hat{\chi}_{\boldsymbol{s}_{0}}(t)=\sum_{i=1}^{n} \lambda_{i}(t) \chi_{\boldsymbol{s}_{i}}(t)=\sum_{i=1}^{n} \boldsymbol{b}_{i}^{\top} \boldsymbol{\phi}_{J}(t) \boldsymbol{\phi}_{J}(t)^{\top} \boldsymbol{a}_{i} \mathrm{e}$

$$
\begin{aligned}
\operatorname{Var}\left(\hat{\chi}_{\boldsymbol{s}_{0}}(t)-\chi_{\boldsymbol{s}_{0}}(t)\right) & =\sum_{i=1}^{n} \sum_{j=1}^{n} \operatorname{Cov}\left(\boldsymbol{b}_{i}^{\top} \boldsymbol{\phi}_{J}(t) \boldsymbol{\phi}_{J}(t)^{\top} \boldsymbol{a}_{i} ; \boldsymbol{b}_{j}^{\top} \boldsymbol{\phi}_{J}(t) \boldsymbol{\phi}_{J}(t)^{\top} \boldsymbol{a}_{j}\right)+\boldsymbol{\phi}_{J}(t)^{\top} \operatorname{Var}\left(\boldsymbol{a}_{0}\right) \boldsymbol{\phi}_{J}(t)- \\
& -2 \sum_{i=1}^{n} \operatorname{Cov}\left(\boldsymbol{b}_{i}^{\top} \boldsymbol{\phi}_{J}(t) \boldsymbol{\phi}_{J}(t)^{\top} \boldsymbol{a}_{i} ; \boldsymbol{a}_{0} \boldsymbol{\phi}_{J}(t)\right) \\
& =\sum_{i=1}^{n} \sum_{j=1}^{n} \boldsymbol{b}_{i}^{\top} \boldsymbol{\phi}_{J}(t) \boldsymbol{\phi}_{J}(t)^{\top} \operatorname{Cov}\left(\boldsymbol{a}_{i}, \boldsymbol{a}_{j}\right) \boldsymbol{\phi}_{J}(t)^{\top} \boldsymbol{\phi}_{J}(t) \boldsymbol{b}_{j}^{\top}+\boldsymbol{\phi}_{J}(t)^{\top} \operatorname{Var}\left(\boldsymbol{a}_{0}\right) \boldsymbol{\phi}_{J}(t)- \\
& -2 \sum_{i=1}^{n} \boldsymbol{b}_{i}^{\top} \boldsymbol{\phi}_{J}(t) \boldsymbol{\phi}_{J}(t)^{\top} \operatorname{Cov}\left(\boldsymbol{a}_{i}, \boldsymbol{a}_{0}\right) \boldsymbol{\phi}_{J}(t)
\end{aligned}
$$

Considere as seguintes notações

$$
\begin{aligned}
Q_{i, j} & =\int_{0}^{1} \boldsymbol{\phi}_{J}(t) \boldsymbol{\phi}_{J}(t)^{\top} \operatorname{Cov}\left(\boldsymbol{a}_{i}, \boldsymbol{a}_{j}\right) \boldsymbol{\phi}_{J}(t)^{\top} \boldsymbol{\phi}_{J}(t) d t, \quad i, j \in\{i, \ldots, n\}, \\
D & =\boldsymbol{\phi}_{J}(t)^{\top} \operatorname{Var}\left(\boldsymbol{a}_{0}\right) \boldsymbol{\phi}_{J}(t), \\
\boldsymbol{P}_{i} & =\int_{0}^{1} \boldsymbol{\phi}_{J}(t) \boldsymbol{\phi}_{J}(t)^{\top} \operatorname{Cov}\left(\boldsymbol{a}_{i}, \boldsymbol{a}_{0}\right) \boldsymbol{\phi}_{J}(t) d t, \quad i \in\{1, \ldots, n\},
\end{aligned}
$$

então o problema de otimização (3.6) pode ser reescrito em

$$
\boldsymbol{b}_{1}, \ldots, \boldsymbol{b}_{n}=\arg \min _{\boldsymbol{b}_{1}, \ldots, \boldsymbol{b}_{n}} \sum_{i=1}^{n} \sum_{j=1}^{n} \boldsymbol{b}_{i}^{\top} Q_{i j} \boldsymbol{b}_{j}+D-2 \sum_{i=1}^{n} \boldsymbol{b}_{i}^{\top} \boldsymbol{P}_{i} \quad \text { sujeito a } \sum_{i=1}^{n} \boldsymbol{b}_{i}=\boldsymbol{\alpha}
$$

Usando multiplicadores de Lagrange, temos que

$$
\boldsymbol{b}_{1}, \ldots, \boldsymbol{b}_{n}=\arg \min _{\boldsymbol{b}_{1}, \ldots, \boldsymbol{b}_{n}} \sum_{j=1}^{n} \boldsymbol{b}_{i}^{\top} Q_{i j} \boldsymbol{b}_{j}+D-2 \sum_{i=1}^{n} \boldsymbol{b}_{i}^{\top} \boldsymbol{P}_{i}+2 \boldsymbol{m}^{\top}\left(\sum_{i=1}^{n} \boldsymbol{b}_{i}-\boldsymbol{\alpha}\right)
$$


como o termo $D$ é constante em relação a $\boldsymbol{b}_{1}, \ldots, \boldsymbol{b}_{n}$, ele não interfere na busca pelo ponto de mínimo e podemos reescrever a equação 3.8 na seguinte forma matricial

$$
\left(\boldsymbol{b}_{1}^{\top}, \ldots, \boldsymbol{b}_{n}^{\top}, \boldsymbol{m}^{\top}\right)^{\top}=\arg \min \boldsymbol{\beta}^{\top} Q \boldsymbol{\beta}-2 \boldsymbol{\beta} \boldsymbol{P},
$$

em que,

$$
\begin{aligned}
\boldsymbol{\beta} & =\left(\boldsymbol{b}_{1}^{\top}, \ldots, \boldsymbol{b}_{n}^{\top}, \boldsymbol{m}^{\top}\right)^{\top}, \\
Q & =\left(\begin{array}{ccccc}
Q_{11} & Q_{12} & \ldots & Q_{1 n} & I_{N} \\
Q_{21} & Q_{22} & \ldots & Q_{2 n} & I_{N} \\
\vdots & \vdots & \ddots & \vdots & \vdots \\
Q_{n 1} & Q_{n 2} & \ldots & Q_{n n} & I_{N} \\
I_{N} & I_{N} & \ldots & I_{N} & \mathbf{0}_{N}
\end{array}\right), \\
\boldsymbol{P} & =\left(\boldsymbol{P}_{1}^{\top}, \ldots, \boldsymbol{P}_{n}^{\top}, \boldsymbol{c}^{\top}\right)^{\top} .
\end{aligned}
$$

Note que no Teorema 3.2.1 é necessário estimar a matriz de covariância cruzada $\operatorname{Cov}\left(\boldsymbol{a}_{i}, \boldsymbol{a}_{j}\right)$ de um campo aleatório multivariado fracamente estacionário ${ }^{1}$, passo que envolve técnicas elaboradas de estimação, pois precisamos garantir que as estimativas sejam positiva-definidas. Para os leitores interessados, Genton e Kleiber (2015) faz um revisão de métodos para estimar a matriz covariância cruzada de um campo aleatório estacionário. Contudo, é possível contornar esse desafio usando o semivariograma de um campo aleatório de uma maneira análoga ao proposto no Teorema 3.1.1 usando o Teorema 3.2.2.

Teorema 3.2.2. Suponha que $\boldsymbol{a}_{0}, \boldsymbol{a}_{1}, \ldots, \boldsymbol{a}_{n}$ são observações de um campo aleatório multivariado fracamente estacionário e isotrópico, então resolver o problema de otimização descrito por

$$
\arg \min _{\boldsymbol{b}_{1}, \ldots, \boldsymbol{b}_{n}} \mathrm{E}\left[\left\|\hat{\chi}_{\boldsymbol{s}_{0}}(t)-\boldsymbol{\chi}_{\boldsymbol{s}_{0}}(t)\right\|^{2}\right] \text { sujeito } a \sum_{i=1}^{n} \lambda_{i}(t)=\boldsymbol{\alpha}^{\top} \boldsymbol{\phi}_{J}(t),
$$

\footnotetext{
${ }^{1}$ Vide, por exemplo, Goulard e Voltz (1992) para uma revisão sobre campo aleatório fracamente estacionário e isotrópico.
} 
em que $\hat{\chi}_{\boldsymbol{s}_{0}}=\sum_{i=1}^{n} \boldsymbol{b}_{i}^{\top} \boldsymbol{\phi}_{J}(t) \boldsymbol{\phi}_{J}(t)^{\top} \boldsymbol{a}_{i}, \lambda_{i}(t)=\boldsymbol{b}_{i}^{\top} \boldsymbol{\phi}_{J}(t)$ e $\boldsymbol{\alpha}^{\top} \boldsymbol{\phi}_{J}(t) \approx 1, \forall t \in[0,1]$ é equivalente a encontrar o ponto de máximo da seguinte forma quadrática

$$
\boldsymbol{\beta}^{\top} R \boldsymbol{\beta}-2 \boldsymbol{U}^{\top} \boldsymbol{\beta}
$$

em que

$$
\begin{aligned}
\boldsymbol{\beta} & =\left(\boldsymbol{b}_{1}^{\top}, \ldots, \boldsymbol{b}_{n}^{\top}, \boldsymbol{m}^{\top}\right)^{\top}, \\
R & =\left(\begin{array}{ccccc}
R_{11} & R_{12} & \ldots & R_{1 n} & I_{N} \\
R_{21} & R_{22} & \ldots & R_{2 n} & I_{N} \\
\vdots & \vdots & \ddots & \vdots & \vdots \\
R_{n 1} & R_{n 2} & \ldots & R_{n n} & I_{N} \\
I_{N} & I_{N} & \ldots & I_{N} & \mathbf{0}_{N}
\end{array}\right), \\
\boldsymbol{U} & =\left(\boldsymbol{U}_{1}^{\top}, \ldots, \boldsymbol{U}_{n}^{\top}, \boldsymbol{\alpha}^{\top}\right)^{\top},
\end{aligned}
$$

com $N$ a ordem da matriz $R_{11} e$

$$
\begin{aligned}
R_{i j} & =\int_{0}^{1} \boldsymbol{\phi}_{J}(t) \boldsymbol{\phi}_{J}(t)^{\top} \frac{1}{2} \operatorname{Var}\left(\boldsymbol{a}_{i}-\boldsymbol{a}_{j}\right) \boldsymbol{\phi}_{J}(t) \boldsymbol{\phi}_{J}(t)^{\top} d t, \quad i, j \in\{1, \ldots, n\}, \\
\boldsymbol{U}_{i} & =\int_{0}^{1} \boldsymbol{\phi}(t) \boldsymbol{\phi}_{J}(t)^{\top} \frac{1}{2} \operatorname{Var}\left(\boldsymbol{a}_{i}-\boldsymbol{a}_{0}\right) \boldsymbol{\phi}_{J}(t) d t, \quad i \in\{1, \ldots, n\}, \\
\boldsymbol{\alpha}^{\top} \boldsymbol{\phi}_{J}(t) & \approx \begin{cases}1, & \text { se } t \in[0,1] \\
0, & \text { caso contrário }\end{cases}
\end{aligned}
$$

Demonstração. Por hipótese, $\boldsymbol{a}_{0}, \boldsymbol{a}_{1}, \ldots, \boldsymbol{a}_{n}$ são observações de um campo aleatório multivariado fracamente estacionário e isotrópico, isto é,

$$
\begin{aligned}
\operatorname{Cov}\left(\boldsymbol{a}_{i}, \boldsymbol{a}_{j}\right) & =C\left(\left\|\boldsymbol{s}_{i}-\boldsymbol{s}_{j}\right\|\right), \quad i, j \in\{0,1, \ldots, n\}, \\
\frac{1}{2} \operatorname{Var}\left(\boldsymbol{a}_{i}-\boldsymbol{a}_{j}\right) & =G\left(\left\|\boldsymbol{s}_{i}-\boldsymbol{s}_{j}\right\|\right), \quad i, j \in\{0,1, \ldots, n\}, \\
C\left(\left\|\boldsymbol{s}_{i}-\boldsymbol{s}_{j}\right\|\right) & =C(0)-G\left(\left\|\boldsymbol{s}_{i}-\boldsymbol{s}_{j}\right\|\right), \quad i, j \in\{0,1, \ldots, n\},
\end{aligned}
$$

em que $G(h)$ é o semivariograma para o campo multivariado. 
De acordo com o Teorema 3.2.1, resolver o problema de otimização

$$
\arg \min _{\boldsymbol{b}_{1}, \ldots, \boldsymbol{b}_{n}} \mathrm{E}\left[\left\|\hat{\chi}_{\boldsymbol{s}_{0}}(t)-\boldsymbol{\chi}_{\boldsymbol{s}_{0}}(t)\right\|\right] \text { sujeito a } \sum_{i=1}^{n} \lambda_{i}(t)=1
$$

$\operatorname{com} \lambda_{i}(t)=\boldsymbol{b}_{i}^{\top} \phi_{J}(t)$, é equivalente a achar o ponto de mínimo da forma quadrática

$$
\boldsymbol{\beta}^{\top} Q \boldsymbol{\beta}-2 \boldsymbol{\beta}^{\top} \boldsymbol{P}
$$

com

$$
\begin{aligned}
Q & =\left(\begin{array}{ccccc}
Q_{11} & Q_{12} & \ldots & Q_{1 n} & I_{N} \\
Q_{21} & Q_{22} & \ldots & Q_{2 n} & I_{N} \\
\vdots & \vdots & \ddots & \vdots & I_{N} \\
Q_{n 1} & Q_{n 2} & \ldots & Q_{n n} & I_{N} \\
I_{N} & I_{N} & \ldots & I_{N} & \mathbf{0}_{N}
\end{array}\right), \\
\boldsymbol{P} & =\left(\boldsymbol{P}_{1}^{\top}, \ldots, \boldsymbol{P}_{n}^{\top}, \boldsymbol{\alpha}^{\top}\right)^{\top},
\end{aligned}
$$

em que

$$
\begin{aligned}
Q_{i j} & =\int_{0}^{1} \boldsymbol{\phi}_{J}(t) \boldsymbol{\phi}_{J}(t)^{\top} C\left(\left\|\boldsymbol{s}_{i}-\boldsymbol{s}_{j}\right\|\right) \boldsymbol{\phi}_{J}(t) \boldsymbol{\phi}_{J}(t)^{\top} d t, \quad i, j \in\{0,1, \ldots, n\}, \\
\boldsymbol{P}_{i} & =\int_{0}^{1} \boldsymbol{\phi}_{J}(t) \boldsymbol{\phi}_{J}(t)^{\top} C\left(\left\|\boldsymbol{s}_{i}-\boldsymbol{s}_{0}\right\|\right) \boldsymbol{\phi}_{J}(t) d t, \quad i \in\{0,1, \ldots, n\}, \\
\boldsymbol{\beta} & =\left(\boldsymbol{b}_{1}^{\top}, \ldots, \boldsymbol{b}_{n}^{\top}, \boldsymbol{m}^{\top}\right)^{\top} .
\end{aligned}
$$

Como $C\left(\left\|s_{i}-s_{j}\right\|\right)=C(0)-G\left(\left\|s_{i}-s_{j}\right\|\right)$, temos que

$$
\begin{aligned}
Q_{i j} & =\int_{0}^{1} \boldsymbol{\phi}_{J}(t) \phi_{J}(t)^{\top} C\left(\left\|\boldsymbol{s}_{i}-\boldsymbol{s}_{j}\right\|\right) \boldsymbol{\phi}_{J}(t) \boldsymbol{\phi}_{J}(t)^{\top} d t \\
& =\int_{0}^{1} \boldsymbol{\phi}_{J}(t) \boldsymbol{\phi}_{J}(t)^{\top}\left[C(0)-G\left(\left\|\boldsymbol{s}_{i}-\boldsymbol{s}_{j}\right\|\right)\right] \boldsymbol{\phi}_{J}(t) \boldsymbol{\phi}_{J}(t)^{\top} d t \\
& =Q_{00}-R_{i j},
\end{aligned}
$$


$\mathrm{e}$

$$
\begin{aligned}
\boldsymbol{P}_{i} & =\int_{0}^{1} \boldsymbol{\phi}_{J}(t) \boldsymbol{\phi}_{J}(t)^{\top} C\left(\left\|\boldsymbol{s}_{i}-\boldsymbol{s}_{0}\right\|\right) \boldsymbol{\phi}_{J}(t) d t \\
& =\int_{0}^{1} \boldsymbol{\phi}_{J}(t) \boldsymbol{\phi}_{J}(t)^{\top}\left[C(0)-G\left(\left\|\boldsymbol{s}_{i}-\boldsymbol{s}_{0}\right\|\right)\right] \boldsymbol{\phi}_{J}(t) d t \\
& =\boldsymbol{P}_{0}-\boldsymbol{U}_{i} .
\end{aligned}
$$

Logo, $Q=Q^{0}-R$ e $P=P^{0}-U$ com

$$
\begin{aligned}
Q^{0} & =\left(\begin{array}{cc}
\left(\mathbf{1 1}^{\top}\right) \otimes Q_{00} & \mathbf{1} \otimes \mathbf{0} \\
\mathbf{1}^{\top} \otimes \mathbf{0} & \mathbf{0}
\end{array}\right), \\
P^{0} & =\left(\begin{array}{c}
\mathbf{1} \otimes \boldsymbol{P}_{0} \\
\mathbf{0} \cdot \mathbf{1}
\end{array}\right) .
\end{aligned}
$$

em que $\mathbf{1}=(1, \ldots, 1)^{\top}$ é um vetor coluna $N$ linhas e $\mathbf{0}$ é uma matriz nula de ordem $N \times N$. Observe que

$$
\begin{aligned}
\boldsymbol{\beta}^{\top} Q^{0} \boldsymbol{\beta} & =\left(\left(\sum_{i=1}^{n} \boldsymbol{b}_{i}^{\top}\right) Q_{00}, \ldots,\left(\sum_{i=1}^{n} \boldsymbol{b}_{i}^{\top}\right) Q_{00}, \mathbf{1}^{\top} \cdot \mathbf{0}\right)\left(\begin{array}{c}
\boldsymbol{b}_{1} \\
\vdots \\
\boldsymbol{b}_{n} \\
\mathbf{0} \cdot \mathbf{1}
\end{array}\right) \\
& =\sum_{j=1}^{n} \sum_{i=1}^{n} \boldsymbol{b}_{i}^{\top} Q_{00} \boldsymbol{b}_{j} \\
& =\boldsymbol{\alpha}^{\top} Q_{00} \boldsymbol{\alpha}
\end{aligned}
$$

e

$$
\boldsymbol{P}^{0^{\top}} \boldsymbol{\beta}=\boldsymbol{P}_{0}^{\top} \sum_{i=1}^{n} \boldsymbol{b}_{i}=\boldsymbol{P}_{0}^{\top} \boldsymbol{\alpha} .
$$


Finalmente, temos que

$$
\begin{aligned}
\arg \min _{\boldsymbol{b}_{1}, \ldots, \boldsymbol{b}_{n}}\left[\boldsymbol{\beta}^{\top} Q \boldsymbol{\beta}-2 \boldsymbol{\beta P}\right] & =\arg \min _{\boldsymbol{b}_{1}, \ldots, \boldsymbol{b}_{n}}\left[\boldsymbol{\beta}^{\top} Q^{0} \boldsymbol{\beta}-\boldsymbol{\beta}^{\top} R \boldsymbol{\beta}-2 \boldsymbol{P}_{0}^{\top} \boldsymbol{\beta}+2 \boldsymbol{\beta}^{\top} \boldsymbol{U}\right] \\
& =\arg \min _{\boldsymbol{b}_{1}, \ldots, \boldsymbol{b}_{n}}\left[\boldsymbol{\alpha} Q_{00} \boldsymbol{\alpha}-\boldsymbol{\beta}^{\top} R \boldsymbol{\beta}-2 \boldsymbol{P}_{0}^{\top} \boldsymbol{\alpha}+2 \boldsymbol{\beta}^{\top} \boldsymbol{U}\right] \\
& =\arg \max _{\boldsymbol{b}_{1}, \ldots, \boldsymbol{b}_{n}}\left[\boldsymbol{\beta}^{\top} R \boldsymbol{\beta}-2 \boldsymbol{\beta}^{\top} \boldsymbol{U}\right] .
\end{aligned}
$$

O Teorema 3.2.2 substitui a necessidade de estimação da matriz covariância cruzada $\operatorname{Cov}\left(\boldsymbol{a}_{i}, \boldsymbol{a}_{j}\right)$ no modelo proposto por Henao et al. (2010) pela estimação do semivariograma $\frac{1}{2} \operatorname{Var}\left(\boldsymbol{a}_{i}-\boldsymbol{a}_{j}\right)$ que pode ser estimada via estimador não paramétrico apresentado por Goulard e Voltz (1992):

$$
G(h)=\frac{1}{2|N(h)|} \sum_{(i, j) \in N(h)}\left[\boldsymbol{a}_{i}-\boldsymbol{a}_{j}\right]\left[\boldsymbol{a}_{i}-\boldsymbol{a}_{j}\right]^{\top}
$$

em que $N(h)=\left\{(i, j) \in\{1, \ldots, n\} \times\{1, \ldots, n\} \mid\left\|\boldsymbol{s}_{i}-\boldsymbol{s}_{j}\right\| \in(h-\epsilon, h+\epsilon)\right\}$ e $\epsilon>0$ é uma banda de tolerância (vide Wackernagel, 2003, para maiores detalhes).

De uma forma geral, a função escala $\phi(t)$ em uma análise de multirresolução não tem forma analítica fechada. Para resolver as integrais nas equações de $R_{i, j}$ e $\boldsymbol{U}_{i}$, usamos uma aproximação motivada pela integração de Riemann: $\phi\left(\frac{l}{2^{q}}\right)$ é conhecido para $l, q \in \mathbb{Z}$ (vide Boggess e Narcowich, 2009, para maiores detalhes), então temos que

i. $\int_{0}^{1} \boldsymbol{\phi}_{J}(t) \boldsymbol{\phi}_{J}^{\top}(t) G\left(\left\|\boldsymbol{s}_{i}-\boldsymbol{s}_{j}\right\|\right) \boldsymbol{\phi}_{J}(t) \boldsymbol{\phi}_{J}^{\top}(t) d t \approx \sum_{k \in C} \frac{1}{2^{q}} \boldsymbol{\phi}_{J}\left(\frac{k}{2^{q}}\right) \boldsymbol{\phi}_{J}^{\top}\left(\frac{k}{2^{q}}\right)$.

$$
\cdot G\left(\left\|s_{i}-s_{j}\right\|\right) \phi_{J}\left(\frac{k}{2^{q}}\right) \phi_{J}^{\top}\left(\frac{k}{2^{q}}\right) \operatorname{com} C=\left\{k \in \mathbb{Z} \mid \frac{k}{2^{q}} \in[0,1]\right\}
$$

ii. $\int_{0}^{1} \phi_{J}(t) \phi_{J}^{\top}(t) G\left(\left\|\boldsymbol{s}_{i}-\boldsymbol{s}_{j}\right\|\right) \boldsymbol{\phi}_{J}(t) d t \approx \sum_{k \in C} \frac{1}{2^{q}} \phi_{J}\left(\frac{k}{2^{q}}\right) \boldsymbol{\phi}_{J}^{\top}\left(\frac{k}{2^{q}}\right) G\left(\left\|\boldsymbol{s}_{i}-\boldsymbol{s}_{j}\right\|\right) \boldsymbol{\phi}_{J}\left(\frac{k}{2^{q}}\right)$ $\operatorname{com} C=\left\{k \in \mathbb{Z} \mid \frac{k}{2^{q}} \in[0,1]\right\}$.

A Figura 3.2 ilustra a ideia das aproximações i. e ii.. 


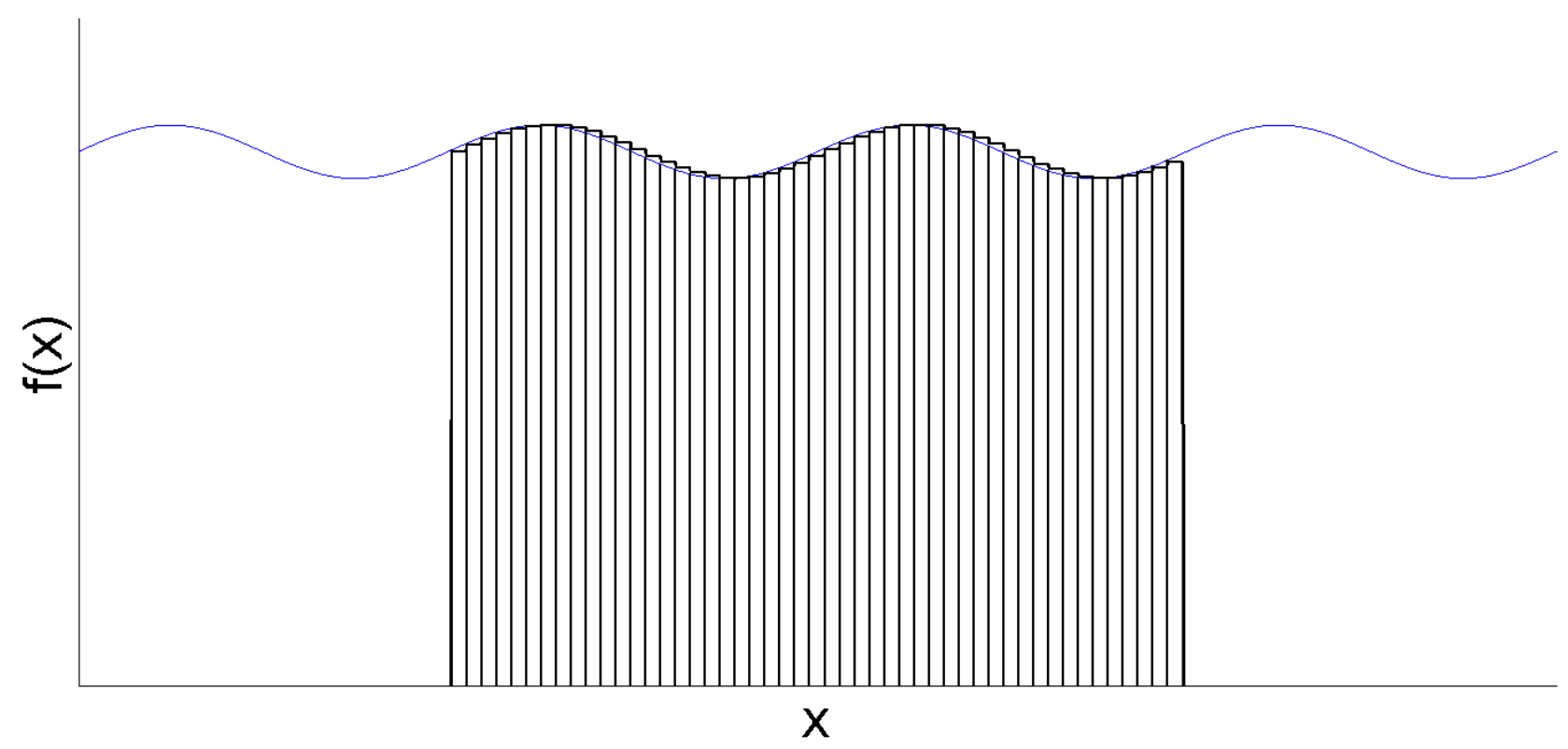

Figura 3.2: Ilustração do método numérico utilizado nas integrais envolvendo a Krigagem Tempo-Variante Funcional.

\subsection{Krigagem Funcional por Campo}

Nessa seção introduzimos uma abordagem de interpolação espacial distinta daquelas apresentadas na Seção 3.1 e na Seção 3.2. Em vez de buscarmos estimar a curva $\chi_{\boldsymbol{s}_{0}}(t)$ como combinação linear (envolvendo valores escalares ou funções) das curvas $\chi_{\boldsymbol{s}_{i}}(t)$ em nossa amostra funcional, desejamos estimar os coeficientes $\boldsymbol{a}_{0}$ da projeção de $\chi_{\boldsymbol{s}_{0}}(t)$ no espaço de aproximação $V_{J}$. Mais precisamente, suponha que aproximamos as curvas $\chi_{\boldsymbol{s}_{1}}(t), \ldots, \chi_{\boldsymbol{s}_{n}}(t)$ por

$$
\chi_{\boldsymbol{s}_{i}}(t) \approx \boldsymbol{a}_{i}^{\top} \boldsymbol{\phi}_{J}(t), \quad i=1, \ldots, n,
$$

em que $\boldsymbol{a}_{i}=\left(a_{i,-M}^{J}, \ldots, a_{i, M}^{J}\right)^{\top}$ e $\phi_{J}(t)=\left(\phi_{J,-M}(t), \ldots, \phi_{J, M}(t)\right)^{\top}$. Nosso objetivo é encontrar uma estimativa $\hat{\boldsymbol{a}}_{0}$ para $\boldsymbol{a}_{0}$ e, consequentemente, obter uma estimativa para $\chi_{\boldsymbol{s}_{0}}(t)$ através de $\hat{\chi}_{\boldsymbol{s}_{0}}=$ $\hat{\boldsymbol{a}}_{0}^{\top} \phi_{J}(t)$.

A ideia para obter uma estimativa de $\boldsymbol{a}_{0}$ é usar uma combinação linear de $\boldsymbol{a}_{1}, \ldots, \boldsymbol{a}_{n}$, mas em vez de usar a mesma constante escalar $\theta_{i}$ para cada coordenada $a_{i,-M}^{J}, \ldots, a_{i, M}^{J}$ da projeção de $\chi_{\boldsymbol{s}_{i}}(t)$ em $V_{J}$, optamos por atribuir uma constante $\theta_{i, j}$ para cada $a_{i, j}^{J}, i=1, \ldots, n, j=$ $-M, \ldots, M$. Analogamente aos estimadores descritos na Seção 3.1 e na Seção 3.2, desejamos encontrar $\theta_{i, j}, i=1, \ldots, n, j=-M, \ldots, M$ com erro quadrático médio mínimo e não viciado. Formalmente, suponha que $\boldsymbol{a}_{0}, \boldsymbol{a}_{1}, \ldots, \boldsymbol{a}_{n}$ são observações de um campo aleatório multivariado 
fracamente estacionário e isotrópico e considere as matrizes

$$
\Theta_{i}=\left(\begin{array}{cccc}
\theta_{i,-M} & 0 & \ldots & 0 \\
0 & \theta_{i,-M+1} & \ldots & 0 \\
\vdots & \vdots & \ddots & \vdots \\
0 & 0 & \ldots & \theta_{i M}
\end{array}\right), \quad i=1, \ldots, n .
$$

Então, um estimador para $\boldsymbol{a}_{0}$ é dado por

$$
\hat{\boldsymbol{a}}_{0}=\sum_{i=1}^{n} \Theta_{i} \boldsymbol{a}_{i}
$$

em que $\Theta_{1}, \ldots, \Theta_{n}$ é solução do problema de otimização descrito por

$$
\begin{aligned}
& \Theta_{1}, \ldots, \Theta_{n}=\arg \min _{\Theta_{1}, \ldots, \Theta_{n}} \mathrm{E}\left[\left(\sum_{i=1}^{n} \Theta_{i} \boldsymbol{a}_{i}-\boldsymbol{a}_{0}\right)^{\top}\left(\sum_{i=1}^{n} \Theta_{i} \boldsymbol{a}_{i}-\boldsymbol{a}_{0}\right)\right] \\
& \text { sujeito a } \mathrm{E}\left(\sum_{i=1}^{n} \Theta_{i} \boldsymbol{a}_{i}-\boldsymbol{a}_{0}\right)=\mathbf{0}
\end{aligned}
$$

em que $\mathbf{0}=(0, \ldots, 0)^{\top}$ é um vetor coluna nulo com a mesma dimensão de $\boldsymbol{a}_{i}$. Logo, uma estimativa para a curva em $s_{0}$ é dada por

$$
\hat{\chi}_{\boldsymbol{s}_{0}}(t)=\hat{\boldsymbol{a}}_{0}^{\top} \boldsymbol{\phi}_{J}(t)
$$

Denominamos o interpolador espacial de curva descrito pela equação 3.13 de método de Krigagem Funcional por Campo. A escolha desse nome se deve ao fato que supomos que os coeficientes da projeção da curvas no espaço de aproximação em nossa amostra funcional constituem um campo aleatório isotrópico e fracamente estacionário.

Teorema 3.3.1. Seja $\boldsymbol{a}_{0}, \boldsymbol{a}_{1}, \ldots, \boldsymbol{a}_{n}$ observações de um campo aleatório multivariado estacionário e isotrópico. Então, resolver o problema de otimização descrito por $\arg \min _{\Theta_{1}, \ldots, \Theta_{n}} \mathrm{E}\left[\left(\sum_{i=1}^{n} \Theta_{i} \boldsymbol{a}_{i}-\boldsymbol{a}_{0}\right)^{\top}\left(\sum_{i=1}^{n} \Theta_{i} \boldsymbol{a}_{i}-\boldsymbol{a}_{0}\right)\right]$ sujeito a $\mathrm{E}\left(\sum_{i=1}^{n} \Theta_{i} \boldsymbol{a}_{i}-\boldsymbol{a}_{0}\right)=\mathbf{0}$, 
é equivalente a encontrar o ponto de máximo da forma quadrática

$$
\boldsymbol{\beta}^{\top} A \boldsymbol{\beta}-2 \boldsymbol{\beta}^{\top} \boldsymbol{B}
$$

em que

$$
\begin{aligned}
\boldsymbol{\beta}= & \left(\boldsymbol{\theta}_{1}^{\top}, \ldots, \boldsymbol{\theta}_{n}^{\top}, \boldsymbol{\delta}^{\top}\right)^{\top}, \\
\boldsymbol{B}= & \left(\left(G\left(\boldsymbol{a}_{1}, \boldsymbol{a}_{0}\right) \circ I\right) \cdot \mathbf{1}, \ldots,\left(G\left(\boldsymbol{a}_{n}, \boldsymbol{a}_{0}\right) \circ I\right) \cdot \mathbf{1},-\mathbf{1}^{\top}\right)^{\top}, \\
A= & \left(\begin{array}{ccccc}
G\left(\boldsymbol{a}_{1}, \boldsymbol{a}_{1}\right) \circ I & G\left(\boldsymbol{a}_{1}, \boldsymbol{a}_{2}\right) \circ I & \ldots & G\left(\boldsymbol{a}_{1}, \boldsymbol{a}_{n}\right) \circ I & I \\
G\left(\boldsymbol{a}_{2}, \boldsymbol{a}_{1}\right) \circ I & G\left(\boldsymbol{a}_{2}, \boldsymbol{a}_{2}\right) \circ I & \ldots & G\left(\boldsymbol{a}_{2}, \boldsymbol{a}_{n}\right) \circ I & I \\
\vdots & \vdots & \ddots & \vdots & \vdots \\
G\left(\boldsymbol{a}_{n}, \boldsymbol{a}_{1}\right) \circ I & G\left(\boldsymbol{a}_{n}, \boldsymbol{a}_{2}\right) \circ I & \ldots & G\left(\boldsymbol{a}_{n}, \boldsymbol{a}_{n}\right) \circ I & I \\
I & I & \ddots & I & \mathbf{0}
\end{array}\right),
\end{aligned}
$$

em que $\boldsymbol{\theta}_{i}=\left(\theta_{i,-M}, \ldots, \theta_{i, M}\right)^{\top}$, $\boldsymbol{\delta}$ é um vector coluna de mesma dimensão de $\boldsymbol{\theta}_{i}$, о é o produto de Hadamard (vide Styan, 1973, para maiores detalhes), $G\left(\boldsymbol{a}_{i}, \boldsymbol{a}_{j}\right)=\frac{1}{2} \operatorname{Var}\left(\boldsymbol{a}_{i}-\boldsymbol{a}_{j}\right), 0$ é matriz nula com mesma dimensão de $G\left(\boldsymbol{a}_{i}, \boldsymbol{a}_{i}\right)$ e I é a matriz identidade com a mesma ordem de $G\left(\boldsymbol{a}_{i}, \boldsymbol{a}_{j}\right)$

Demonstração. Primeiro note que, sob a restrição $\mathrm{E}\left(\sum_{i=1}^{n} \Theta_{i} \boldsymbol{a}_{i}-\boldsymbol{a}_{0}\right)=\mathbf{0}$, temos que

$$
\mathrm{E}\left(\sum_{i=1}^{n} \Theta_{i} \boldsymbol{a}_{i}-\boldsymbol{a}_{0}\right)^{\top}\left(\sum_{i=1}^{n} \Theta_{i} \boldsymbol{a}_{i}-\boldsymbol{a}_{0}\right)=\operatorname{traço}\left[\operatorname{Var}\left(\sum_{i=1}^{n} \Theta_{i} \boldsymbol{a}_{i}-\boldsymbol{a}_{0}\right)\right] .
$$

Observe que a restrição $\mathrm{E}\left(\sum_{i=1}^{n} \Theta_{i} \boldsymbol{a}_{i}-\boldsymbol{a}_{0}\right)=\mathbf{0}$ é equivalente a $\sum_{i=1}^{n} \Theta_{i}=I$ e 


$$
\begin{aligned}
\operatorname{Var}\left(\sum_{i=1}^{n} \Theta_{i} \boldsymbol{a}_{i}-\boldsymbol{a}_{0}\right) & =\operatorname{Var}\left(\boldsymbol{a}_{0}\right)+\sum_{i=1}^{n} \Theta_{i} \operatorname{Var}\left(\boldsymbol{a}_{i}\right) \Theta_{i}-2 \sum_{i=1}^{n} \operatorname{Cov}\left(\Theta_{i} \boldsymbol{a}_{i} ; \boldsymbol{a}_{0}\right)+ \\
& +2 \sum_{j=1}^{n} \sum_{k=j+1}^{n} \Theta_{j} \operatorname{Cov}\left(\boldsymbol{a}_{j} ; \boldsymbol{a}_{k}\right) \Theta_{k} \\
& =C(0)-2 \sum_{i=1}^{n} \Theta_{i}\left[C(0)-G\left(\boldsymbol{a}_{i}, \boldsymbol{a}_{0}\right)\right]+\sum_{i=1}^{n} \Theta_{i} C(0) \Theta_{i}+ \\
& +2 \sum_{j=1}^{n} \sum_{k=j+1}^{n} \Theta_{j}\left[C(0)-G\left(\boldsymbol{a}_{j}, \boldsymbol{a}_{k}\right)\right] \Theta_{k} \\
& =C(0)+\sum_{j=1}^{n} \sum_{k=1}^{n} \Theta_{j} C(0) \Theta_{k}-2 C(0)+2 \sum_{i=1}^{n} \Theta_{i} G\left(\boldsymbol{a}_{i}, \boldsymbol{a}_{0}\right) \\
& -\sum_{j=1}^{n} \sum_{k=1}^{n} \Theta_{j} G\left(\boldsymbol{a}_{j}, \boldsymbol{a}_{k}\right) \Theta_{k} \\
& =2 \sum_{i=1}^{n} \Theta_{i} G\left(\boldsymbol{a}_{i}, \boldsymbol{a}_{0}\right)-\sum_{j=1}^{n} \sum_{k=1}^{n} \Theta_{j} G\left(\boldsymbol{a}_{j}, \boldsymbol{a}_{k}\right) \Theta_{k} .
\end{aligned}
$$

Logo, o problema de otimização 3.12 é equivalente a

$\arg \min _{\Theta_{1}, \ldots, \Theta_{n}} 2 \sum_{i=1}^{n} \operatorname{traço}\left(\Theta_{i} G\left(\boldsymbol{a}_{i}, \boldsymbol{a}_{0}\right)\right)-\sum_{j=1}^{n} \sum_{k=1}^{n} \operatorname{traço}\left(\Theta_{j} G\left(\boldsymbol{a}_{j}, \boldsymbol{a}_{k}\right) \Theta_{k}\right)$ sujeito a $\sum_{i=1}^{n} \Theta_{i}=I$,

em que traço $(\cdot)$ é o traço de uma matriz. Seja $\boldsymbol{\theta}_{i}=\left(\theta_{i,-M}, \ldots, \theta_{i, M}\right)$, então o problema de otimização (3.12) pode ser resumido em

$$
\begin{gathered}
\arg \min _{\boldsymbol{\theta}_{1}, \ldots, \boldsymbol{\theta}_{n}} 2 \sum_{i=1}^{n} \boldsymbol{\theta}_{i}^{\top}\left(G\left(\boldsymbol{a}_{i}, \boldsymbol{a}_{0}\right) \circ I\right) \mathbf{1}-\sum_{j=1}^{n} \sum_{k=1}^{n} \boldsymbol{\theta}_{j}^{\top}\left(G\left(\boldsymbol{a}_{j}, \boldsymbol{a}_{k}\right) \circ I\right) \boldsymbol{\theta}_{k} \\
\text { sujeito a } \sum_{i=1}^{n} \boldsymbol{\theta}_{i}=\mathbf{1},
\end{gathered}
$$

em que o é operador de Hadamard (vide Styan, 1973, para maiores detalhes) e $\mathbf{1}=(1, \ldots, 1)^{\top}$ é vetor coluna com a mesma dimensão de $\boldsymbol{a}_{i}$. Usando multiplicadores de Lagrange, o problema de otimização descrito por (3.12) é equivalente a encontrar o ponto de mínimo de

$$
2 \sum_{i=1}^{n} \boldsymbol{\theta}_{i}^{\top}\left[G\left(\boldsymbol{a}_{i}, \boldsymbol{a}_{0}\right) \circ I\right] \mathbf{1}-\sum_{j=1}^{n} \sum_{k=1}^{n} \boldsymbol{\theta}_{j}^{\top}\left(G\left(\boldsymbol{a}_{j}, \boldsymbol{a}_{k}\right) \circ I\right) \boldsymbol{\theta}_{k}+2 \boldsymbol{\delta}^{\top}\left(\sum_{j=1}^{n} \boldsymbol{\theta}_{j}-\mathbf{1}\right),
$$


em que $\boldsymbol{\delta}$ é um vetor coluna de mesma dimensão de $\boldsymbol{\theta}_{i}$. Em forma matricial temos que

$$
\begin{aligned}
\left(\boldsymbol{\theta}_{1}^{\top}, \ldots, \boldsymbol{\theta}_{n}^{\top}, \boldsymbol{\delta}^{\top}\right)^{\top} & =\arg \min _{\boldsymbol{\beta}} 2 \boldsymbol{\beta}^{\top} \boldsymbol{B}-\boldsymbol{\beta}^{\top} A \boldsymbol{\beta} \\
& =\arg \max _{\boldsymbol{\beta}} \boldsymbol{\beta}^{\top} A \boldsymbol{\beta}-2 \boldsymbol{\beta}^{\top} \boldsymbol{B},
\end{aligned}
$$

onde

$$
\begin{aligned}
\boldsymbol{\beta}= & \left(\boldsymbol{\theta}_{1}^{\top}, \ldots, \boldsymbol{\theta}_{n}^{\top}, \boldsymbol{\delta}^{\top}\right)^{\top}, \\
\boldsymbol{B}= & \left(\left(G\left(\boldsymbol{a}_{1}, \boldsymbol{a}_{0}\right) \circ I\right) \cdot \mathbf{1}, \ldots,\left(G\left(\boldsymbol{a}_{n}, \boldsymbol{a}_{0}\right) \circ I\right) \cdot \mathbf{1},-\mathbf{1}^{\top}\right)^{\top}, \\
A= & \left(\begin{array}{ccccc}
G\left(\boldsymbol{a}_{1}, \boldsymbol{a}_{1}\right) \circ I & G\left(\boldsymbol{a}_{1}, \boldsymbol{a}_{2}\right) \circ I & \ldots & G\left(\boldsymbol{a}_{1}, \boldsymbol{a}_{n}\right) \circ I & I \\
G\left(\boldsymbol{a}_{2}, \boldsymbol{a}_{1}\right) \circ I & G\left(\boldsymbol{a}_{2}, \boldsymbol{a}_{2}\right) \circ I & \ldots & G\left(\boldsymbol{a}_{2}, \boldsymbol{a}_{n}\right) \circ I & I \\
\vdots & \vdots & \ddots & \vdots & \vdots \\
G\left(\boldsymbol{a}_{n}, \boldsymbol{a}_{1}\right) \circ I & G\left(\boldsymbol{a}_{n}, \boldsymbol{a}_{2}\right) \circ I & \ldots & G\left(\boldsymbol{a}_{n}, \boldsymbol{a}_{n}\right) \circ I & I \\
I & I & \ldots & I & \mathbf{0}
\end{array}\right) .
\end{aligned}
$$

Note que a forma quadrática na Equação (3.14) no Teorema 3.3.1 não envolve integrações, como nos métodos apresentados na Seção 3.1 e na Seção 3.2, evitando possíveis erros de aproximação numérica. Observe também que transformamos o problema de otimização não linear da Equação (3.12) em um problema de encontrar ponto de máximo de uma forma quadrática: uma tarefa analítica e computacionalmente mais viável.

No Capítulo 4, realizamos um estudo de simulação dos três métodos apresentados nesse capítulo, Krigagem Ordinária Funcional, Krigagem Tempo-Variante Funcional e Krigagem Funcional por Campo , com o objetivo de compará-los usando métricas inspiradas no erro quadrático médio. Note que não é preciso estudar o vício, pois em todos métodos de krigagem para curvas apresentados nesse capítulo restringimos nossa busca em estimadores não viciados. 


\section{Capítulo 4}

\section{Estudo de Simulação}

Em todos modelos de krigagem para dados funcionais espaciais expostos no Capítulo 3, supomos que o processo espacial é pontualmente fracamente estacionário e isotrópico. Mais precisamente, para $s, s_{1}, s_{2}$ pertencente a um subconjunto $D$ limitado e de volume positivo contido na área de estudo, temos que

i. $\mathrm{E}\left[\boldsymbol{\chi}_{\boldsymbol{s}}(t)\right]=m(t), \forall t \in[0,1]$;

ii. $\operatorname{Cov}\left(\chi_{s_{1}}(t), \chi_{s_{2}}(t)\right)=C\left(\left\|s_{1}-s_{2}\right\| ; t\right), \forall t \in[0,1]$ em que $\|\cdot\|$ é a geodésica conforme descrita por Banerjee et al. (2014);

iii. $\frac{1}{2} \operatorname{Var}\left(\boldsymbol{\chi}_{\boldsymbol{s}_{1}}(t)-\boldsymbol{\chi}_{\boldsymbol{s}_{2}}(t)\right)=\gamma\left(\left\|\boldsymbol{s}_{1}-\boldsymbol{s}_{2}\right\| ; t\right), \forall t \in[0,1]$. Para $t \in[0,1]$ fixo, $\gamma\left(\left\|\boldsymbol{s}_{1}-\boldsymbol{s}_{2}\right\| ; t\right)$ é chamada de variograma.

Adicionalmente, em nosso estudo de simulação, supomos que para $t \in[0,1]$ fixo, temos que

$$
\left(\begin{array}{c}
\boldsymbol{\chi}_{\boldsymbol{s}_{1}}(t) \\
\vdots \\
\boldsymbol{\chi}_{\boldsymbol{s}_{n}}(t)
\end{array}\right) \sim N\left(\left(\begin{array}{c}
m(t) \\
\vdots \\
m(t)
\end{array}\right) ; \Sigma(t)\right)
$$

em que $\Sigma(t)$ é uma matriz cuja i-ésima linha e j-ésima coluna é dada por $\sigma_{i, j}(t)=C\left(\left\|\boldsymbol{s}_{i}-\boldsymbol{s}_{j}\right\| ; t\right)$ e $\boldsymbol{s}_{1}, \ldots, \boldsymbol{s}_{n} \in D$.

Usamos os seguintes modelos para a média e a covariância em nosso estudo de simulação: 
a) A função de covariância ( modelo exponencial (Banerjee et al., 2014)):

$$
C(h, t)=\sigma^{2} \exp (-\phi(t) \cdot h), \quad t \in[0,1], h \geq 0,
$$

com $\sigma^{2}=0,005$ e $\phi(t)=10 \sqrt{\frac{t+1}{2}}$. Na Figura 4.1, mostramos o gráfico da função de covariância $C(h, t)$.

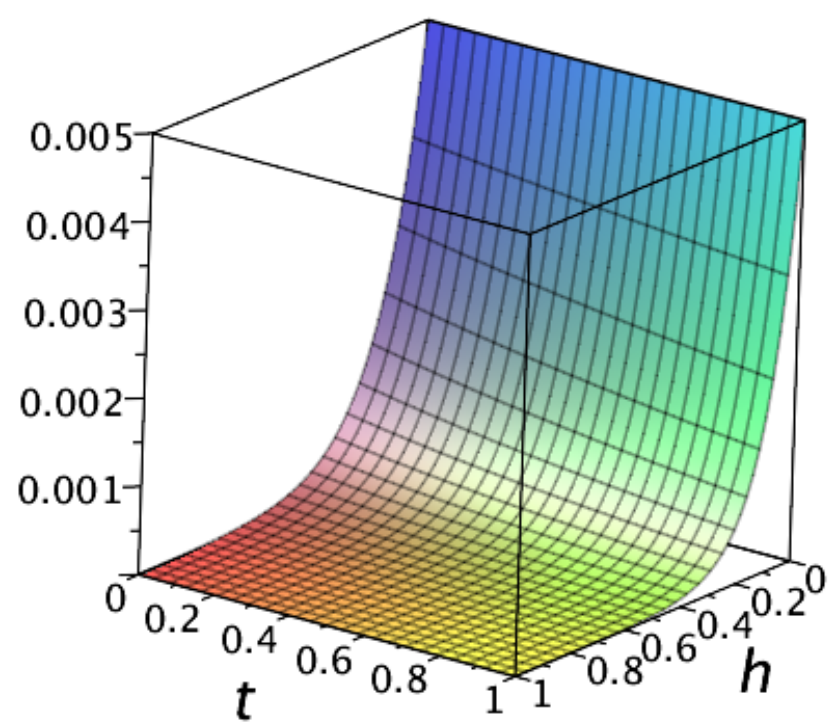

Figura 4.1: Gráfico da função de covariância.

b) Foram consideradas duas funções médias. Uma função média contínua dada por

$$
m_{1}(t)=\sin (2 \pi t)
$$

e outra função média constante por partes com descontinuidades em $\left\{\frac{1}{3}, \frac{2}{3}\right\}$ dada por

$$
m_{2}(t)=\left\{\begin{array}{ll}
0.5, & t \in\left[0, \frac{1}{3}\right) \cup\left[\frac{2}{3}, 1\right] . \\
1, & t \in\left[\frac{1}{3}, \frac{2}{3}\right)
\end{array} .\right.
$$

Na construção das amostras funcionais, primeiramente, geramos 50 pontos na região ilustrada na Figura 4.2. Estes pontos são os mesmos para todas as amostras funcionais simuladas. 
Após estabelecidos os pontos $\boldsymbol{s}_{1}, \ldots, \boldsymbol{s}_{50}$, consideramos $t_{k}=\frac{k-1}{2^{9}}, k=1, \ldots, 2^{9}$ e para cada $t_{k}$ geramos 50 valores da distribuição normal multivariada com média $\left(m\left(t_{k}\right), \ldots, m\left(t_{k}\right)\right)^{\top}$ e matriz de covariância $\Sigma\left(t_{k}\right)$.

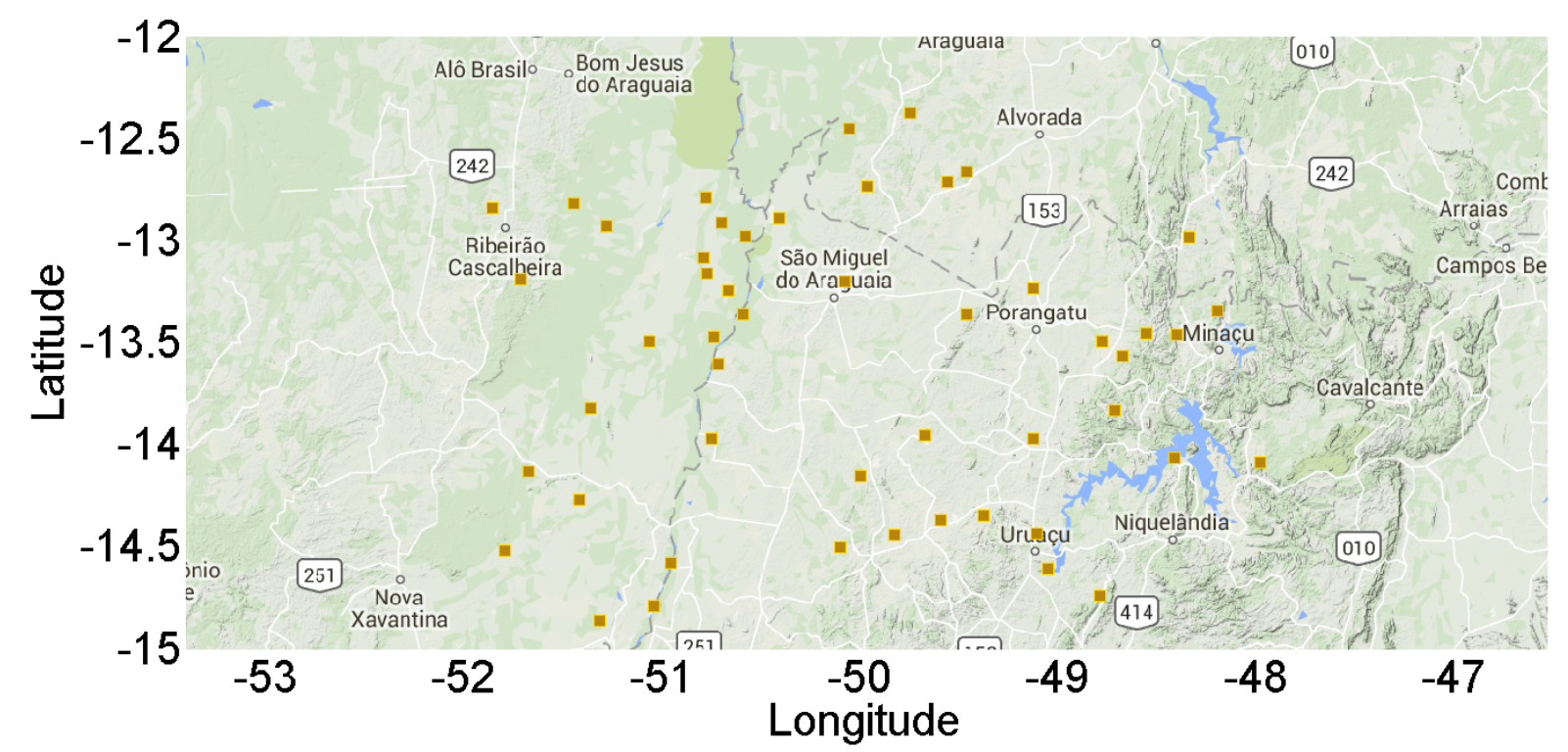

Figura 4.2: Região onde os dados foram simulados. Cada ponto amarelo é uma localidade selecionada em nosso estudo de simulação.

\subsection{Medidas de Qualidade de Ajuste}

Considere um contexto em que temos $M=100$ amostras funcionais com 50 curvas cada. Então, obtemos $M$ estimativas $\chi_{\boldsymbol{s}_{0}}^{1}(t), \ldots, \chi_{\boldsymbol{s}_{0}}^{M}(t)$ para a curva $\chi_{\boldsymbol{s}_{0}}(t)$ no ponto não monitorado $\boldsymbol{s}_{0}$. Em ordem para avaliar o desempenho dos três métodos de Krigagem introduzidos no Capítulo 3, Krigagem Ordinária Funcional, Krigagem Tempo-Variante Funcional e Krigagem Funcional por Campo, usamos duas medidas de comparação: erro quadrático médio e erro quadrático por amostra, descritas a seguir.

\subsubsection{Erro Quadrático Médio}

Podemos estimar o erro quadrático médio dos interpoladores espaciais estudados nesse trabalho usando uma aproximação para integral inspirada na integração de Riemann. Mais precisamente, 


$$
\begin{aligned}
\mathrm{E}\left\|\hat{\chi}_{\boldsymbol{s}_{0}}(t)-\chi_{\boldsymbol{s}_{0}}(t)\right\|^{2} & =\mathrm{E} \int_{0}^{1}\left|\hat{\chi}_{\boldsymbol{s}_{0}}(t)-\chi_{\boldsymbol{s}_{0}}(t)\right|^{2} d t \\
& =\int_{0}^{1} \mathrm{E}\left[\hat{\chi}_{\boldsymbol{s}_{0}}(t)-\chi_{\boldsymbol{s}_{0}}(t)\right]^{2} d t \\
& \approx \int_{0}^{1} \frac{1}{M} \sum_{k=1}^{M}\left|\hat{\chi}_{\boldsymbol{s}_{0}}^{k}(t)-\chi_{\boldsymbol{s}_{0}}(t)\right|^{2} \\
& \approx \sum_{l=2}^{2^{9}}\left(t_{l}-t_{l-1}\right) \frac{1}{M} \sum_{k=1}^{M}\left|\hat{\chi}_{\boldsymbol{s}_{0}}^{k}\left(t_{l}\right)-\chi_{\boldsymbol{s}_{0}}\left(t_{l}\right)\right|^{2} \\
& =\frac{1}{2^{9} M} \sum_{l=2}^{2^{9}} \sum_{k=1}^{M}\left|\hat{\chi}_{\boldsymbol{s}_{0}}^{k}\left(t_{l}\right)-\chi_{\boldsymbol{s}_{0}}\left(t_{l}\right)\right|^{2},
\end{aligned}
$$

em que $t_{l} \in[0,1], l=1, \ldots, 2^{9}$ são pontos igualmente espaçados com $t_{l}-t_{l-1}=\frac{1}{2^{9}}$. Usamos a notação $E Q M$ para essa medida.

\subsubsection{Erro Quadrático Médio por Amostra}

Para avaliar a performance dos métodos de krigagem em cada amostra, calculamos a norma em $L^{2}(\mathbb{R})$ da diferença entre a curva estimada $\chi_{\boldsymbol{s}_{0}}^{k}(t)$ e a verdadeira curva observada $\chi_{\boldsymbol{s}_{0}}(t)$ e usamos a notação $E Q M_{k}$ para essa medida. Mais precisamente,

$$
E Q M_{k}=\int_{0}^{1}\left|\chi_{\boldsymbol{s}_{0}}^{k}(t)-\chi_{\boldsymbol{s}_{0}}(t)\right|^{2} d t
$$

A integral na equação (4.2) é aproximada por retângulos de uma maneira análoga a Seção 4.1.1, isto é

$$
\begin{aligned}
E Q M_{k} & =\int_{0}^{1}\left|\chi_{\boldsymbol{s}_{0}}^{k}(t)-\chi_{\boldsymbol{s}_{0}}(t)\right|^{2} d t \\
& \approx \sum_{l=2}^{2^{9}}\left(t_{l}-t_{l-1}\right)\left|\chi_{\boldsymbol{s}_{0}}^{k}\left(t_{l}\right)-\chi_{\boldsymbol{s}_{0}}\left(t_{l}\right)\right|^{2} \\
& =\frac{1}{2^{9}} \sum_{l=2}^{2^{9}}\left|\chi_{\boldsymbol{s}_{0}}^{k}\left(t_{l}\right)-\chi_{\boldsymbol{s}_{0}}\left(t_{l}\right)\right|^{2}
\end{aligned}
$$


em que $t_{l} \in[0,1], l=1, \ldots, 2^{9}$ são pontos igualmente espaçados com $t_{l}-t_{l-1}=\frac{1}{2^{9}}$.

\subsection{Resultado - Simulação}

Nessa seção, analisamos empiricamente os métodos de Krigagem para o contexto de Dados Funcionais propostos no Capítulo 3: Krigagem Ordinária Funcional, Krigagem Tempo-Variante Funcional e Krigagem Funcional por Campo. Dividimos nosso estudo de simulação em dois contextos: quando o ponto não monitorado $s_{0}$ está no centro da região sob estudo e quando o ponto não monitorado $s_{1}$ está afastado e / ou no canto da região. Na Figura 4.3, exibimos os 50 pontos selecionados espaciais para simular as amostras funcionais e os pontos $s_{0}$ e $s_{1}$ onde desejamos aplicar os métodos de krigagem para estimar uma curva. Desejamos com isso avaliar o desempenho dos métodos propostos quando existem várias curvas próximas e em volta do ponto não monitorado e quando há poucas curvas ao redor. Ademais, usamos três bases nesse estudo de simulação: ondaletas Haar, ondaletas Daubechies com $N=3$ ou brevemente $d b 3$ e B-Splines que foi a base usada nos trabalhos originais de Henao et al. (2010) e Henao et al. (2011). Para a média constante usamos Haar cujas ondaletas pai e mãe são constantes por parte e para o média contínua usamos a ondaleta $d b 3$ cujas ondaletas pai e mãe são também contínuas. Os resultados obtidos são comparados com BSplines que foi empregado nos trabalhos originais de Henao (2009); Henao et al. (2010, 2011). Organizamos essa seção em duas subseções: na primeira seção analisamos o comportamento dos modelos para média contínua $m_{1}(t)$ para o ponto centralizado $s_{0}$ e para o ponto no canto $s_{1}$ e na segunda seção verificamos a performance dos métodos de krigagem para a média constante por partes $m_{2}(t)$ para os pontos $s_{0}$ e $s_{1}$.

Para facilitar a elaboração de gráficos e tabelas, usamos as seguintes notações:

i. Krigagem Ordinária Funcional abreviamos por KOF,

ii. Krigagem Tempo-Variante Funcional abreviamos por KTVF,

iii. Krigagem Funcional por Campo abreviamos por KFC. 


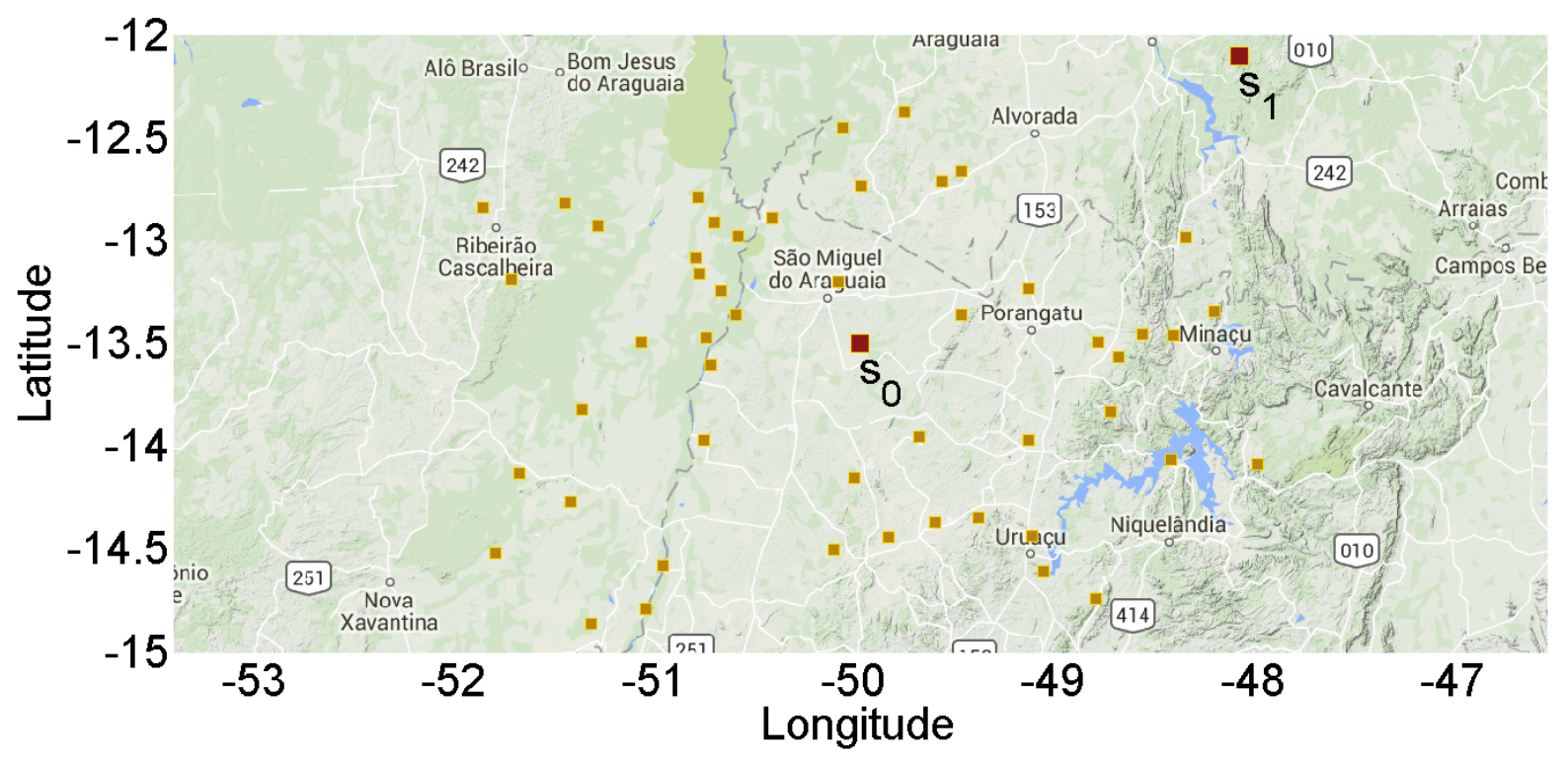

Figura 4.3: Os pontos amarelos indicam as localidades das curvas nas amostras funcionais simuladas. Os pontos vermelhos $s_{0}$ e $s_{1}$ são os pontos não monitorados onde desejamos interpolar usando krigagem.

\subsubsection{Simulação 1: Média Contínua}

Erro Quadrático Médio para o ponto centralizado $s_{0}$ e para o ponto no canto $s_{1}$ é mostrado nas tabelas 4.1 e 4.2, respectivamente. Primeiramente, ressaltamos que o uso de BSplines teve o maior $E Q M$ entre todos os métodos de Krigagem analisados. Além disso, o método Krigagem Funcional por Campo obteve o menor valor de EQM, chegando a ser entre $10 \%$ e $20 \%$ menor do que os métodos estabelecidos na literatura que usam BSplines para Krigagem Ordinária Funcional e Krigagem Tempo-Variante Funcional. Ressaltamos ainda que a performance do método Krigagem Tempo-Variante Funcional apresentou $E Q M$ menor a Krigagem Ordinária Funcional (modelo mais básico), mas um pouco maior que a Krigagem Funcional por Campo (nova proposta de interpolação espacial).

Por outro, analisando o Erro Quadrático Médio por Amostra, notamos que a Krigagem Funcional por Campo é o estimador com menor variabilidade e a menor média segundo as tabelas 4.3 e 4.4 e as implementações com BSPlines apresentaram maior variabilidade e média.

Nas figuras 4.4 e 4.5, apresentamos os diagramas em caixa para o Erro Quadrático Médio por Amostras. Nestes diagramas retiramos os outliers para facilitar a visualização. Esses gráficos reforçam as afirmações sobre a Krigagem Funcional por Campo e ilustram o desempenho intermediário da Krigagem Tempo-Variante Funcional. Além disso, nas tabelas 4.11 e 4.12 observamos que o 
vício ${ }^{1}$ é pequeno em concordância com os teoremas 3.1.1, 3.2.1, 3.2.2 e 3.3.1 que estabelecem os métodos de Krigagem deste trabalho são não viciados.

Tabela 4.1: Erro Quadrático Médio para $s_{0}$.

\begin{tabular}{l|cc|cc}
\hline Método & Base & EQM & Base & EQM \\
\hline KOF & & 0.0049 & Bsplines & 0.0061 \\
KTVF & db3 & 0.0051 & & 0.0058 \\
KFC & & $\mathbf{0 . 0 0 4 9}$ & - & - \\
\hline
\end{tabular}

Abreviações: KOF - Krigagem Ordinária Funcional; KTVF - Krigagem Tempo-Variante Funcional; KFC - Krigagem Funcional por Campo; db3 - ondaleta Daubechies com $N=3$.

Tabela 4.2: Erro Quadrático Médio para $s_{1}$.

\begin{tabular}{l|cc|cc}
\hline Método & Base & EQM & Base & EQM \\
\hline KOF & & 0.0054 & Bsplines & 0.0063 \\
KTVF & db3 & 0.0050 & Bspline & 0.0060 \\
KFC & & $\mathbf{0 . 0 0 4 8}$ & - & - \\
\hline
\end{tabular}

Abreviações: KOF - Krigagem Ordinária Funcional; KTVF - Krigagem Tempo-Variante Funcional; KFC - Krigagem Funcional por Campo; db3 - ondaleta Daubechies com $N=3$.

Tabela 4.3: Sumário para o Erro Quadrático Médio por Amostra para o ponto centralizado $s_{0}$.

\begin{tabular}{l|ccccccc}
\hline Método - Base & Mínimo & 1-Qua & Média & Mediana & 3-Qua & Máximo & DP \\
\hline KOF - db3 & 0.004817 & 0.004846 & 0.004872 & 0.004859 & 0.004882 & 0.005203 & 0.000050 \\
KOF - Bsplines & 0.005320 & 0.005777 & 0.006094 & 0.005823 & 0.005870 & 0.019033 & 0.001507 \\
KTVF - db3 & 0.005048 & 0.005105 & 0.005133 & 0.005120 & 0.005141 & 0.005682 & 0.000073 \\
KTVF - Bsplines & 0.005717 & 0.005780 & 0.005804 & 0.005804 & 0.005830 & 0.005894 & 0.000039 \\
KFC - db3 & 0.004796 & 0.004850 & $\mathbf{0 . 0 0 4 8 6 9}$ & $\mathbf{0 . 0 0 4 8 7 0}$ & 0.004885 & 0.004954 & 0.000029 \\
\hline
\end{tabular}

Abreviações: KOF - Krigagem Ordinária Funcional; KTVF - Krigagem Tempo-Variante Funcional; KFC - Krigagem Funcional por Campo; db3 - ondaleta Daubechies com $N=3$.

\footnotetext{
${ }^{1} \mathrm{O}$ vício por amostra é calculado pela equação $\frac{1}{2^{J}} \sum_{k=1}^{2^{J}}\left(\chi_{s_{0}}\left(\frac{k}{2^{J}}\right)-\hat{\chi}_{s_{0}}\left(\frac{k}{2^{J}}\right)\right)$.
} 
Tabela 4.4: Sumário para o Erro Quadrático Médio por Amostra para o ponto no canto $s_{1}$.

\begin{tabular}{l|ccccccc}
\hline Método - Base & Mínimo & 1-Qua & Média & Mediana & 3-Qua & Máximo & DP \\
\hline KOF - db3 & 0.00466 & 0.00476 & 0.00539 & 0.00481 & 0.00493 & 0.02128 & 0.00255 \\
KOF - Bsplines & 0.00539 & 0.00600 & 0.00629 & 0.00605 & 0.00610 & 0.01851 & 0.00141 \\
KTVF - db3 & 0.00481 & 0.00486 & 0.00501 & 0.00488 & 0.00491 & 0.01436 & 0.00097 \\
KTVF - Bsplines & 0.00590 & 0.00599 & 0.00604 & 0.00603 & 0.00608 & 0.00627 & 0.00007 \\
KFC - db3 & 0.00466 & 0.00475 & $\mathbf{0 . 0 0 4 7 9}$ & $\mathbf{0 . 0 0 4 7 8}$ & 0.00483 & 0.00505 & 0.00007
\end{tabular}

Abreviações: KOF - Krigagem Ordinária Funcional; KTVF - Krigagem Tempo-Variante Funcional; KFC - Krigagem Funcional por Campo; db3 - ondaleta Daubechies com $N=3$.

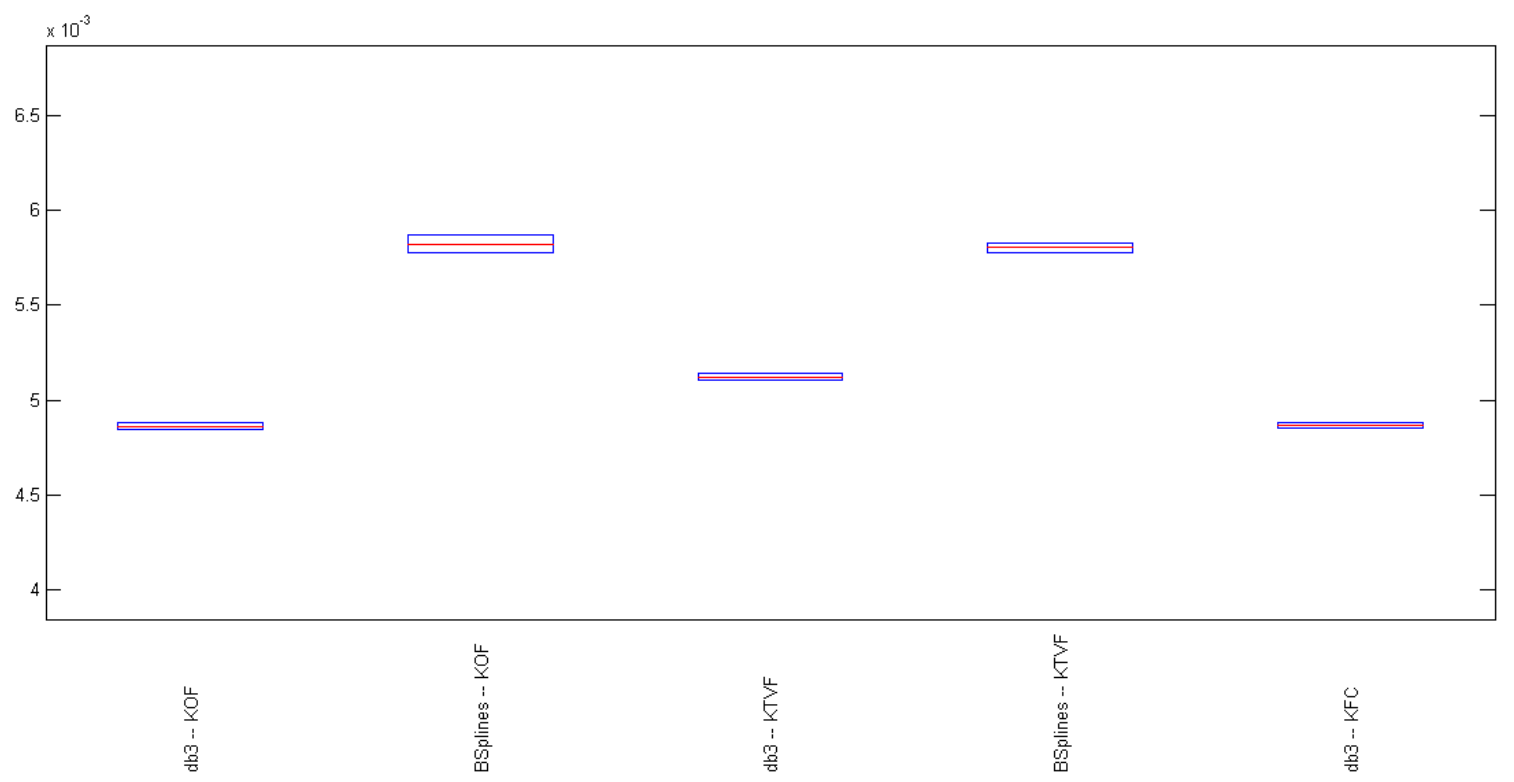

Figura 4.4: Boxplot do Erro Quadrático Médio por Amostra para o ponto no centro da região $s_{0}$ para cada método de krigagem e para cada base no contexto de média contínua.

Tabela 4.5: Sumário para o Vício por Amostra para o ponto centralizado $s_{0}$

\begin{tabular}{l|ccccccc}
\hline Método - Base & Mínimo & 1-Qua & Média & Mediana & 3-Qua & Máximo & DP \\
\hline KOF - db3 & -0.00332 & 0.00057 & 0.00126 & 0.00130 & 0.00212 & 0.00535 & 0.00132 \\
KOF - Bsplines & -0.01253 & -0.00007 & 0.00198 & 0.00091 & 0.00203 & 0.04498 & 0.00692 \\
KTVF - db3 & -0.00325 & 0.00064 & 0.00137 & 0.00141 & 0.00222 & 0.00548 & 0.00132 \\
KTVF - Bsplines & -0.00218 & 0.00035 & 0.00072 & 0.00073 & 0.00128 & 0.00256 & 0.00080 \\
KFC - db3 & -0.00209 & 0.00017 & 0.00090 & 0.00089 & 0.00175 & 0.00346 & 0.00116 \\
\hline
\end{tabular}

Abreviações: KOF - Krigagem Ordinária Funcional; KTVF - Krigagem Tempo-Variante Funcional; KFC - Krigagem Funcional por Campo; db3 - ondaleta Daubechies com $N=3$. 


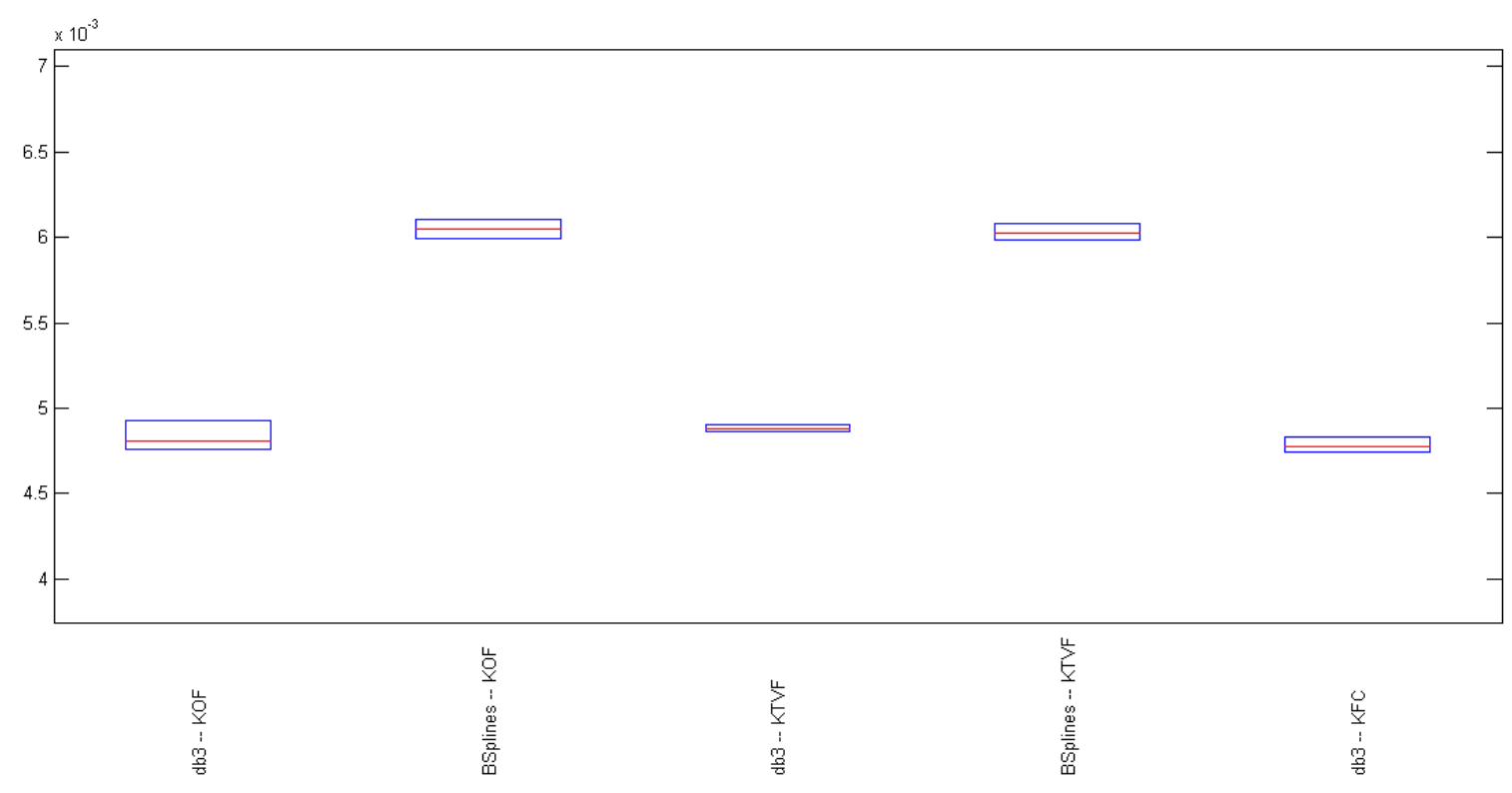

Figura 4.5: Boxplot do Erro Quadrático Médio por Amostra para o ponto no canto da região si para cada método de krigagem e para cada base no contexto de média contínua.

Tabela 4.6: Sumário para o Vício por Amostra para o ponto no canto $s_{1}$

\begin{tabular}{l|ccccccc}
\hline Método - Base & Mínimo & 1-Qua & Média & Mediana & 3-Qua & Máximo & DP \\
\hline KOF - db3 & -0.0277 & -0.0046 & -0.0033 & -0.0024 & -0.0009 & 0.0030 & 0.0047 \\
KOF - Bsplines & -0.0159 & -0.0035 & -0.0014 & -0.0025 & -0.0014 & 0.0416 & 0.0069 \\
KTVF - db3 & -0.0255 & -0.0031 & -0.0024 & -0.0024 & -0.0013 & 0.0031 & 0.0027 \\
KTVF - Bsplines & -0.0057 & -0.0030 & -0.0025 & -0.0026 & -0.0018 & -0.0002 & 0.0011 \\
KFC - db3 & -0.0086 & -0.0040 & -0.0025 & -0.0024 & -0.0010 & 0.0030 & 0.0023 \\
\hline
\end{tabular}

Abreviações: KOF - Krigagem Ordinária Funcional; KTVF - Krigagem Tempo-Variante Funcional; KFC - Krigagem Funcional por Campo; db3 - ondaleta Daubechies com $N=3$. 


\subsubsection{Simulação 2: Média Não Contínua}

Análogo ao caso em que tínhamos média contínua, o método Krigagem Funcional por Campo teve o menor $E Q M$ tanto para o ponto $s_{0}$ e $s_{1}$ conforme tabelas 4.9 e 4.10 . Além disso, quando analisamos também o Erro Quadrático Médio por amostra nos diagramas de caixa ${ }^{2}$ nas figuras 4.4 e 4.7 e nas estatísticas descritivas nas tabelas 4.9 e 4.10 , notamos novamente que interpolação espacial usando Krigagem Funcional por Campo tem desempenho satisfatório e os métodos usando BSplines apresentam a maior mediana e média dessa medida de erro quadrático. Ressaltamos ainda que os melhores métodos usando ondaletas Haar (Krigagem Tempo-Variante Funcional e Krigagem Funcional por Campo) têm Erro Quadrático Médio $16 \%$ menor que o método usando BSplines (Krigagem Tempo-Variante Funcional). Além disso, nas tabelas 4.11 e 4.12 observamos que o vício é pequeno em concordância com os teoremas 3.1.1, 3.2.1, 3.2.2 e 3.3.1 que estabelecem os métodos de Krigagem deste trabalho são não viciados.

Tabela 4.7: Erro Quadrático Médio para $s_{0}$.

\begin{tabular}{l|cc|cc}
\hline Método & Base & EQM & Base & EQM \\
\hline KOF & & 0.01252 & & 0.01463 \\
KTVF & Haar & 0.01212 & Bsplines & 0.01435 \\
KFC & & $\mathbf{0 . 0 1 2 0 7}$ & & - \\
\hline
\end{tabular}

Abreviações: KOF - Krigagem Ordinária Funcional; KTVF - Krigagem Tempo-Variante Funcional; KFC - Krigagem Funcional por Campo.

Tabela 4.8: Erro Quadrático Médio para $s_{1}$.

\begin{tabular}{l|cc|cc}
\hline Método & Base & EQM & Base & EQM \\
\hline KOF & & 0.02640 & & 0.01528 \\
KTVF & Haar & 0.01578 & Bsplines & 0.01502 \\
KFC & & $\mathbf{0 . 0 1 1 9 7}$ & & - \\
\hline
\end{tabular}

Abreviações: KOF - Krigagem Ordinária Funcional; KTVF - Krigagem Tempo-Variante Funcional; KFC - Krigagem Funcional por Campo.

\footnotetext{
${ }^{2}$ Nesses diagramas, retiramos os outliers para facilitar a visualização.
} 
Tabela 4.9: Sumário para o Erro Quadrático Médio por Amostra para o ponto centralizado $s_{0}$.

\begin{tabular}{l|ccccccc}
\hline Método - Base & Mínimo & 1-Qua & Média & Mediana & 3-Qua & Máximo & DP \\
\hline KOF - Haar & 0.012002 & 0.012051 & 0.012514 & 0.012073 & 0.012095 & 0.039457 & 0.002977 \\
KOF - Bsplines & 0.013947 & 0.014315 & 0.014633 & 0.014363 & 0.014432 & 0.029682 & 0.001652 \\
KTVF - Haar & 0.012002 & 0.012050 & 0.012119 & 0.012070 & 0.012090 & 0.013630 & 0.000231 \\
KTVF - Bsplines & 0.014239 & 0.014328 & 0.014351 & 0.014346 & 0.014370 & 0.014465 & 0.000035 \\
KFC - Haar & 0.012015 & 0.012049 & $\mathbf{0 . 0 1 2 0 6 9}$ & $\mathbf{0 . 0 1 2 0 6 6}$ & 0.012088 & 0.012153 & 0.000027 \\
\hline
\end{tabular}

Abreviações: KOF - Krigagem Ordinária Funcional; KTVF - Krigagem Tempo-Variante Funcional; KFC

- Krigagem Funcional por Campo.

Tabela 4.10: Sumário para o Erro Quadrático Médio por Amostra para o ponto centralizado $s_{1}$.

\begin{tabular}{l|ccccccc}
\hline Método - Base & Mínimo & 1-Qua & Média & Mediana & 3-Qua & Máximo & DP \\
\hline KOF - Haar & 0.011848 & 0.011920 & 0.026226 & 0.011983 & 0.012115 & 0.724572 & 0.081861 \\
KOF - Bsplines & 0.014500 & 0.014976 & 0.015275 & 0.015035 & 0.015097 & 0.029605 & 0.001559 \\
KTVF - Haar & 0.011850 & 0.011926 & 0.015780 & 0.011984 & 0.012107 & 0.243905 & 0.025245 \\
KTVF - Bsplines & 0.014827 & 0.014991 & 0.015020 & 0.015025 & 0.015049 & 0.015189 & 0.000059 \\
KFC - Haar & 0.011840 & 0.011926 & $\mathbf{0 . 0 1 1 9 6 5}$ & $\mathbf{0 . 0 1 1 9 6 3}$ & 0.012001 & 0.012115 & 0.000064 \\
\hline
\end{tabular}

Abreviações: KOF - Krigagem Ordinária Funcional; KTVF - Krigagem Tempo-Variante Funcional; KFC

- Krigagem Funcional por Campo.

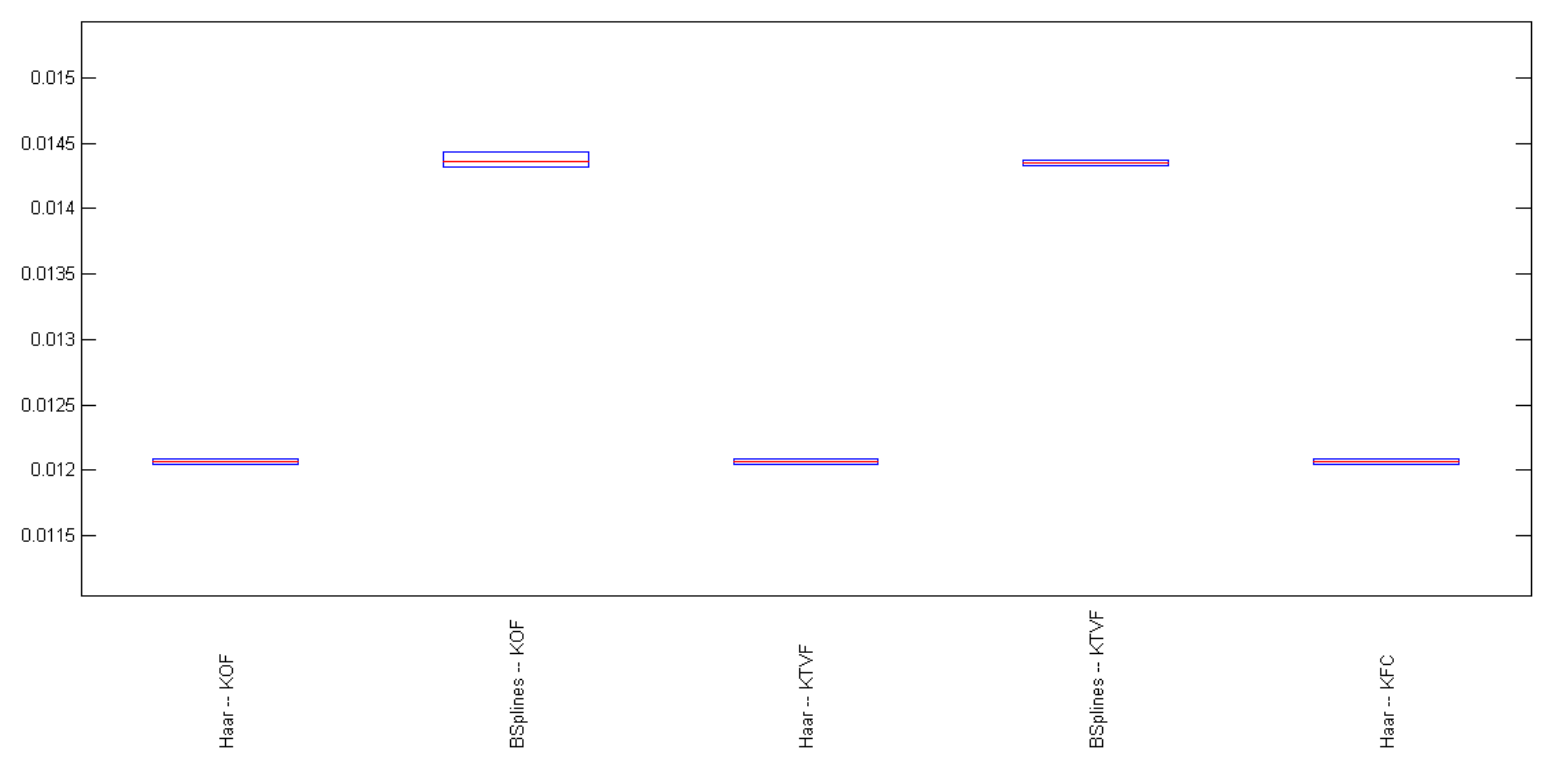

Figura 4.6: Boxplot do Erro Quadrático Médio por Amostra para o ponto no centro da região $s_{0}$ para cada método de krigagem e para cada base no contexto de média não contínua. 


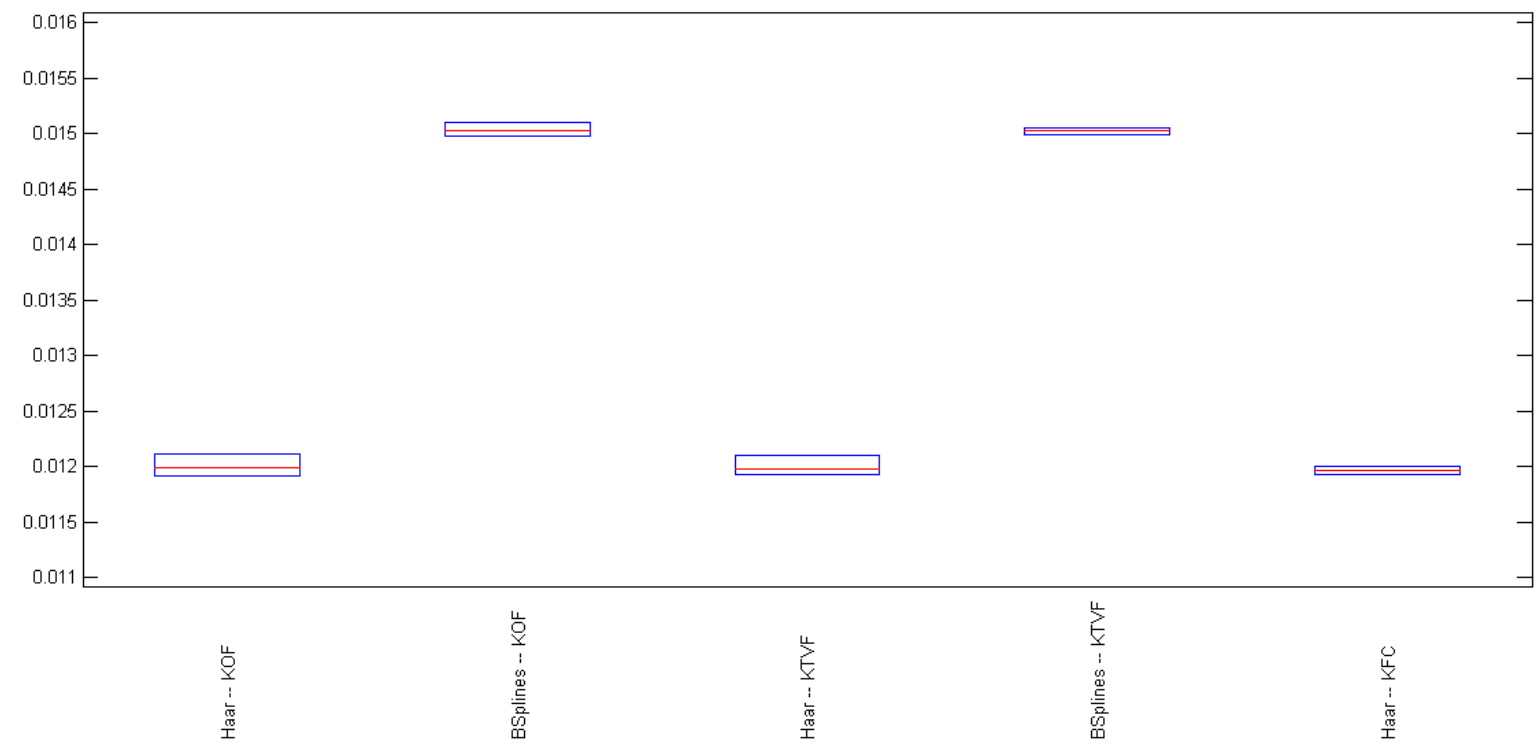

Figura 4.7: Boxplot do Erro Quadrático Médio por Amostra para o ponto no canto da região $s_{1}$ para cada método de krigagem e para cada base no contexto de média não contínua.

Tabela 4.11: Sumário para o Erro Quadrático Médio por Amostra para o ponto no canto $s_{0}$.

\begin{tabular}{l|ccccccc}
\hline Método - Base & Mínimo & 1-Qua & Média & Mediana & 3-Qua & Máximo & DP \\
\hline KOF - Haar & -0.00475 & 0.00070 & 0.00164 & 0.00180 & 0.00267 & 0.01491 & 0.00215 \\
KOF - Bsplines & -0.01592 & -0.00346 & -0.00141 & -0.00248 & -0.00136 & 0.04159 & 0.00692 \\
KTVF - Haar & -0.00467 & 0.00017 & 0.00109 & 0.00121 & 0.00203 & 0.01426 & 0.00205 \\
KTVF - Bsplines & -0.00573 & -0.00303 & -0.00251 & -0.00259 & -0.00181 & -0.00018 & 0.00106 \\
KFC - Haar & -0.00145 & 0.00077 & 0.00142 & 0.00142 & 0.00217 & 0.00482 & 0.00115 \\
\hline
\end{tabular}

Abreviações: KOF - Krigagem Ordinária Funcional; KTVF - Krigagem Tempo-Variante Funcional; KFC - Krigagem Funcional por Campo.

Tabela 4.12: Sumário para o Vício por Amostra para o ponto centralizado $s_{1}$.

\begin{tabular}{l|ccccccc}
\hline Método - Base & Mínimo & 1-Qua & Média & Mediana & 3-Qua & Máximo & DP \\
\hline KOF - db3 & -0.01814 & 0.00499 & 0.00621 & 0.00720 & 0.00867 & 0.01259 & 0.00466 \\
KOF - Bsplines & -0.01592 & -0.00346 & -0.00141 & -0.00248 & -0.00136 & 0.04159 & 0.00692 \\
KTVF - db3 & -0.01819 & 0.00931 & 0.01054 & 0.01145 & 0.01290 & 0.01682 & 0.00470 \\
KTVF - Bsplines & -0.00573 & -0.00303 & -0.00251 & -0.00259 & -0.00181 & -0.00018 & 0.00106 \\
KFC - db3 & 0.00092 & 0.00554 & 0.00705 & 0.00719 & 0.00852 & 0.01256 & 0.00229 \\
\hline
\end{tabular}

Abreviações: KOF - Krigagem Ordinária Funcional; KTVF - Krigagem Tempo-Variante Funcional; KFC

- Krigagem Funcional por Campo. 
Além do novo método proposto nessa tese, denominado Krigagem Funcional por Campo, ter menor Erro Quadrático Médio, ele é computacionalmente mais eficiente que a Krigagem TempoVariante Funcional, conforme gráficos das Figura 4.8. Observamos que as aproximação numéricas na Krigagem Tempo-Variante Funcional tornam esse método consideravelmente menos eficiente que a Krigagem Funcional por Campo. Nas tabelas 4.13(a) e 4.13(b), percebemos que o tempo de execução em segundos da Krigagem Tempo-Variável Funcional é quase três vezes o tempo de execução da Krigagem Funcional por Campo para $n=200$, além do método KTVF ter crescimento ser exponencial. Ressaltamos que melhorias nas aproximações numéricas das integrais podem amenizar este problema, contudo com a Krigagem Funcional por Campo essa questão é contornada.

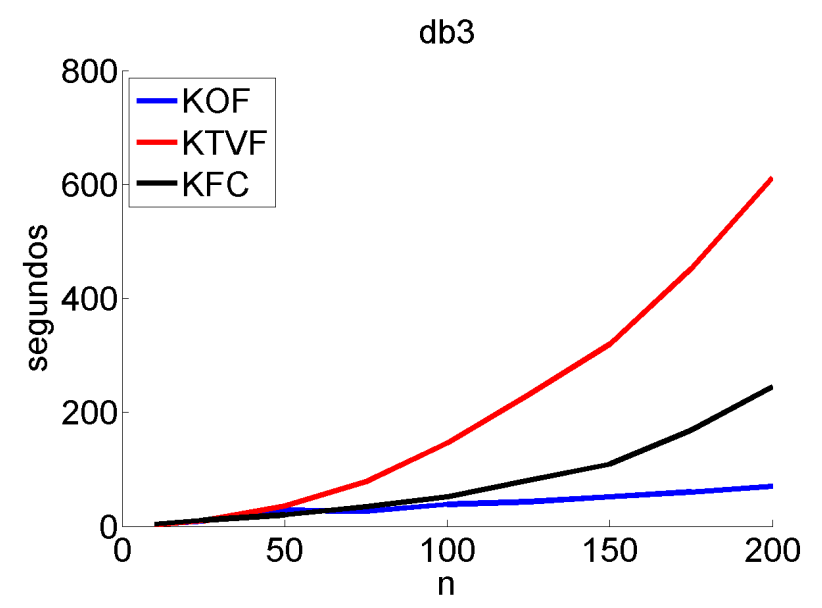

(a) Tempo de execução para ondaleta $d b 3$ em segundos.

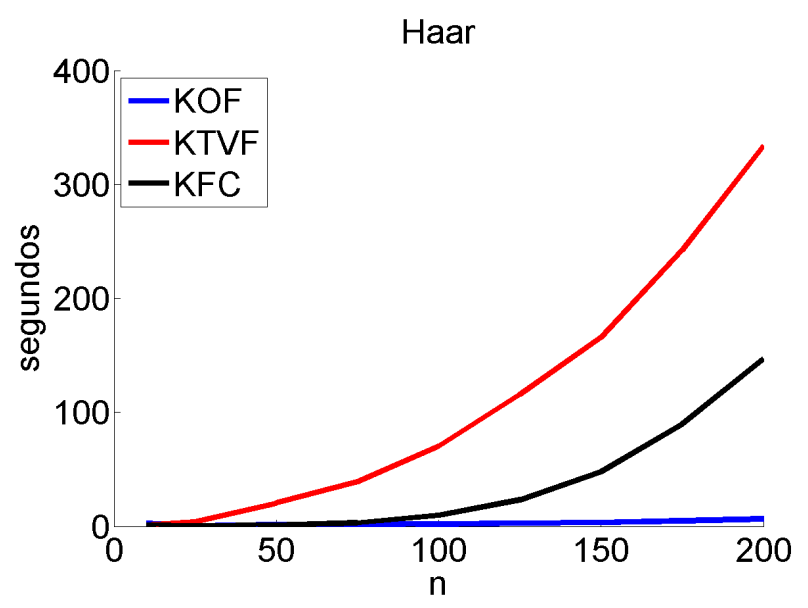

(b) Tempo de execução para ondaleta Haar em segundos.

Abreviações: KOF - Krigagem Ordinária Funcional; KTVF - Krigagem Tempo-Variante Funcional; KFC - Krigagem Funcional por Campo.

Figura 4.8: Comparação do tempo de execução em segundos do métodos de interpolação espacial de curvas, Krigagem Ordinária Funcional, Krigagem Tempo-Variante Funcional e Krigagem Funcional por Campo, usando ondaletas Haar e db3. 
Tabela 4.13: Tempo de execução para os métodos de krigagem, Krigagem Ordinária Funcional (KOF), Krigagem Tempo-Variante Funcional (KTVF) e Krigagem Funcional por Campo (KFC), para vários tamanhos amostrais $n$.

(a) Tempo de execução para ondaletas $d b 3$ em segundos.

\begin{tabular}{l|ccc}
\hline $\mathrm{n}$ & KOF & KTVF & KFC \\
\hline 10 & 3.44 & 2.09 & 3.59 \\
25 & 9.29 & 9.42 & 10.64 \\
50 & 27.97 & 35.62 & 20.08 \\
75 & 26.12 & 78.03 & 34.10 \\
100 & 38.46 & 145.46 & 51.84 \\
125 & 42.87 & 230.73 & 80.29 \\
150 & 51.39 & 319.54 & 109.05 \\
175 & 60.40 & 451.61 & 168.09 \\
200 & 70.43 & 611.44 & 244.60 \\
\hline
\end{tabular}

(b) Tempo de execução para ondaletas Haar em segundos.

\begin{tabular}{l|ccc}
\hline $\mathrm{n}$ & KOF & KTVF & KFC \\
\hline 10 & 2.69 & 1.11 & 1.04 \\
25 & 0.45 & 4.01 & 0.12 \\
50 & 1.22 & 20.31 & 1.04 \\
75 & 1.34 & 39.16 & 3.16 \\
100 & 1.89 & 70.20 & 9.71 \\
125 & 2.78 & 115.76 & 23.23 \\
150 & 3.39 & 165.75 & 47.88 \\
175 & 4.70 & 242.38 & 89.58 \\
200 & 6.63 & 333.89 & 146.70 \\
\hline
\end{tabular}

Abreviações: KOF - Krigagem Ordinária Funcional; KTVF - Krigagem Tempo-Variante Funcional; KFC - Krigagem Funcional por Campo.

Após verificarmos que tanto o uso de ondaletas como o método de Krigagem Funcional por Campo melhoram a interpolação espacial de curvas, apresentamos no Capítulo 5 duas aplicações a dados reais: dados de temperatura média das províncias marítimas canadenses e dados do índice MP10 da região metropolitana de São Paulo. 


\section{Capítulo 5}

\section{Aplicação}

Neste capítulo, ilustramos as três abordagens de krigagem para curvas apresentadas neste trabalho, Krigagem Ordinária Funcional, Krigagem Tempo-Variante Funcional e Krigagem Funcional por Campo, usando dois conjuntos de dados reais: um conjunto de dados de temperatura média no ano de 2000 em 82 estações meteorológicas localizadas nas províncias marítimas canadenses (Nova Scotia, New Brunswick e Prince Edward Island) e dados do índice MP10 da CETESB (Companhia Ambiental do Estado de São Paulo) no ano de 2014 em 14 estações meteorológicas na região metropolitana de São Paulo.

Em cada um dos conjuntos de dados escolhemos uma localidade $s_{i}$, estimamos a curva $\chi_{s_{i}}(t)$ usando as curvas $\chi_{\boldsymbol{s}_{1}}(t), \ldots, \chi_{\boldsymbol{s}_{i-1}}(t), \chi_{\boldsymbol{s}_{i+1}}(t), \ldots, \chi_{\boldsymbol{s}_{n}}(t)$ e criamos um gráfico com a curva interpolada pela krigagem e os valores observados $\chi_{\boldsymbol{s}_{i}}\left(t_{j}\right), j=1, \ldots, 2^{J}$ da curva em $\boldsymbol{s}_{i}$, com o objetivo de ilustrar visualmente a eficácia dos métodos. Além disso, calculamos uma medida de Erro Quadrático Médio dada por

$$
E Q M=\sum_{j=1}^{2^{J}}\left(\hat{\chi}_{\boldsymbol{s}_{i}}\left(t_{j}\right)-\chi_{\boldsymbol{s}_{i}}\left(t_{j}\right)\right)^{2}
$$

em que $\hat{\chi}_{\boldsymbol{s}_{i}}\left(t_{j}\right)$ é a estimativa da curva estimada no instante $t_{j},=1, \ldots, 2^{J}$ usando as curvas $\chi_{\boldsymbol{s}_{1}}(t), \ldots, \chi_{\boldsymbol{s}_{i-1}}(t), \chi_{\boldsymbol{s}_{i+1}}(t), \ldots, \chi_{\boldsymbol{s}_{n}}(t)$. 


\subsection{Temperatura Média Diária das Províncias Marítimas Ca-}

\section{nadenses}

Nessa seção, aplicamos os métodos de Krigagem aos dados climáticos das províncias marítimas do Canadá: New Brunswick, New Scotia e Prince Edward. Em nosso conjunto de dados, consideramos a temperatura média diária registrada em 82 estações meteorológicas das províncias marítimas ao longo do ano de 2000. Na Figura 5.1, mostramos as estações selecionadas em nosso conjunto de dados e na Tabela B.1 do Apêndice B, apresentamos informações tais como ID, nome da estação, latitude e longitude das estações consideradas. Esses dados são bem difundidos na literatura e estão presentes no livro clássico de Ramsay (2006). Conforme Henao et al. (2010), as províncias marítimas cobrem uma área pequena e homogênea que satisfaz as condições de estacionaridade e isotropia descritas na introdução do Capítulo 3. Os dados utilizados nessa seção podem ser facilmente obtidos pelo sítio http://climate.weather.gc.ca/.

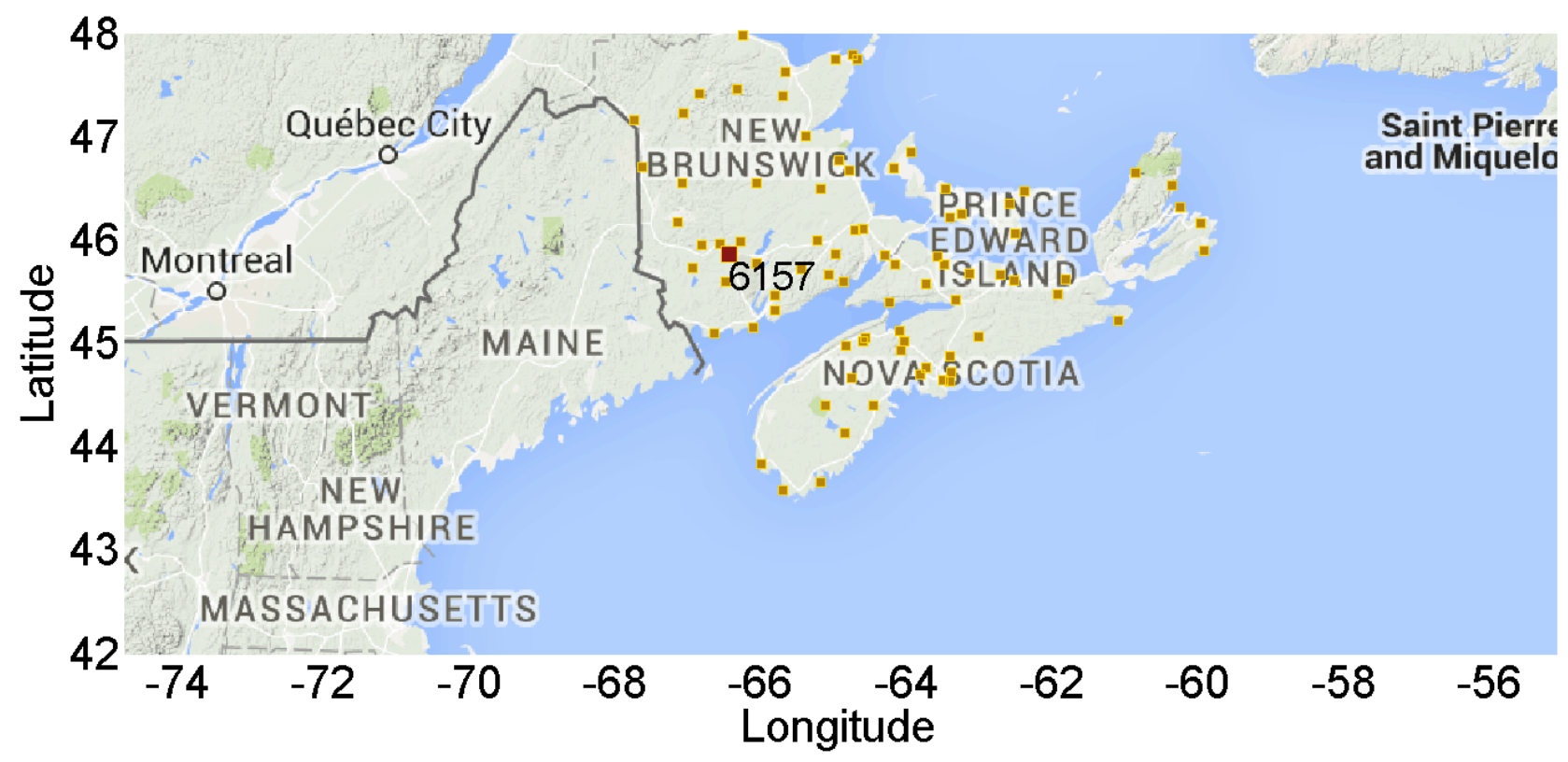

Figura 5.1: Estações meteorológicas selecionadas das províncias marítimas.

Para avaliar a performance dos métodos de Krigagem para a temperatura média diária, escolhemos a estação Fredericon A indicada pelo ID 6157 na Figura 5.1 (quadrado vermelho) e estimamos a temperatura média usando todas as outras estações destacadas no mapa (quadrados amarelos). Temos 82 estação em nosso conjunto de dados e, consequentemente, usamos 81 estações para estimar a curva de temperatura média na estação Fredericon A no ano 2000. Nas 
Figuras 5.2(a) e 5.2(b), mostramos o ajuste da curva pelo modelo de Krigagem Ordinária, Krigagem Tempo-Variante Funcional e Krigagem Funcional por Campo usando ondaletas Haar e db3. Visualmente a Krigagem Tempo-Variante Funcional e a Krigagem Funcional por Campo interpolam melhor os dados do que o método de Krigagem Ordinário Funcional. Este comportamento é confirmado pelo Erro Quadrático Médio apresentado na Tabela 5.1. Repetimos esse processo de estimar a curva $\chi_{\boldsymbol{s}_{i}}(t)$ usando $\chi_{\boldsymbol{s}_{1}}(t), \ldots, \chi_{\boldsymbol{s}_{i-1}}(t), \chi_{\boldsymbol{s}_{i+1}}(t), \ldots, \chi_{\boldsymbol{s}_{n}}(t)$ e calcular $E Q M=\sum_{j=1}^{2^{J}}\left(\hat{\chi}_{\boldsymbol{s}_{i}}\left(t_{j}\right)-\chi_{\boldsymbol{s}_{i}}\left(t_{j}\right)\right)^{2}$ para todas as localidades $\boldsymbol{s}_{i}, i=1, \ldots, n$ e podemos verificar na Tabela 5.2 e na Figura 5.3 que, em média, a Krigagem Tempo-Variante Funcional e a Krigagem Funcional por Campo tem Erro Quadrático Médio menor que a Krigagem Ordinária Funcional.

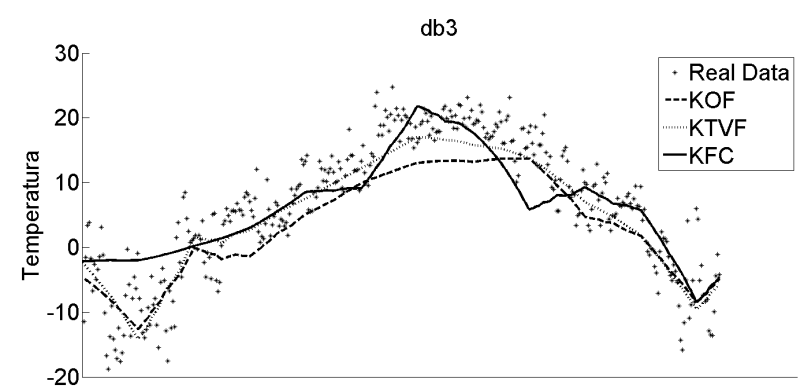

(a) $d b 4$

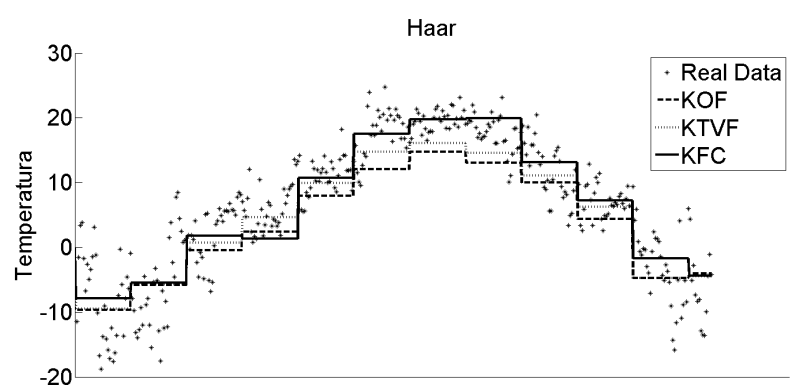

(b) Haar

Abreviações: KOF - Krigagem Ordinária Funcional; KTVF - Krigagem Tempo-Variante Funcional; KFC - Krigagem Funcional por Campo; db3 - ondaleta Daubechies com $N=3$.

Figura 5.2: Curvas ajustadas pelos métodos de interpolação espacial para curvas, Krigagem Ordinária Funcional (KOF), Krigagem Tempo-Variante Funcional e Krigagem Funcional por Campo, usando ondaletas Haar e Daubechies com $N=3$.

Tabela 5.1: Erro Quadrático Médio para a estação Fredericon A.

\begin{tabular}{l|cc|cc}
\hline Método & Ondaleta & EQM & Ondaleta & EQM \\
\hline KOF & & 28.26228 & & 26.02128 \\
KTVF & Haar & $\mathbf{2 2 . 8 6 8 8 2}$ & db3 & $\mathbf{1 8 . 9 3 9 0 3}$ \\
KFC & & 25.312483 & & 22.62712 \\
\hline
\end{tabular}

Abreviações: KOF - Krigagem Ordinária Funcional; KTVF - Krigagem Tempo-Variante Funcional; KFC - Krigagem Funcional por Campo; db3 - ondaleta Daubechies com $N=3$. 
Tabela 5.2: Sumário de EQM para todas estações.

\begin{tabular}{l|cccccccc}
\hline Método - Base & Mínimo & 1-Qua & Média & Mediana & 3-Qua & Máximo & DP & Soma \\
\hline KTVF - db3 & 13.73 & 19.74 & $\mathbf{1 7 5 . 2 4}$ & $\mathbf{3 0 . 4 7}$ & 80.91 & 6844.12 & 768.21 & 14369.42 \\
KTVF - Haar & 15.13 & 22.55 & 188.49 & 34.21 & 79.47 & 3910.21 & 566.19 & 15456.07 \\
KFC - Haar & 20.76 & 36.00 & 266.34 & 95.50 & 231.20 & 2715.57 & 502.78 & 21839.80 \\
KFC - db3 & 19.29 & 28.36 & 279.80 & 48.66 & 213.75 & 4773.36 & 653.29 & 22943.74 \\
KOF - Haar & 15.88 & 24.69 & 312.99 & 42.29 & 109.68 & 6315.45 & 961.14 & 25665.56 \\
KOF - db3 & 14.58 & 22.34 & $8.08 \mathrm{E}+06$ & 41.50 & 120.31 & $6.61 \mathrm{E}+08$ & $7.30 \mathrm{E}+07$ & $6.62 \mathrm{E}+08$ \\
\hline
\end{tabular}

Abreviações: KOF - Krigagem Ordinária Funcional; KTVF - Krigagem Tempo-Variante Funcional; KFC

- Krigagem Funcional por Campo; db3 - ondaleta Daubechies com $N=3$.

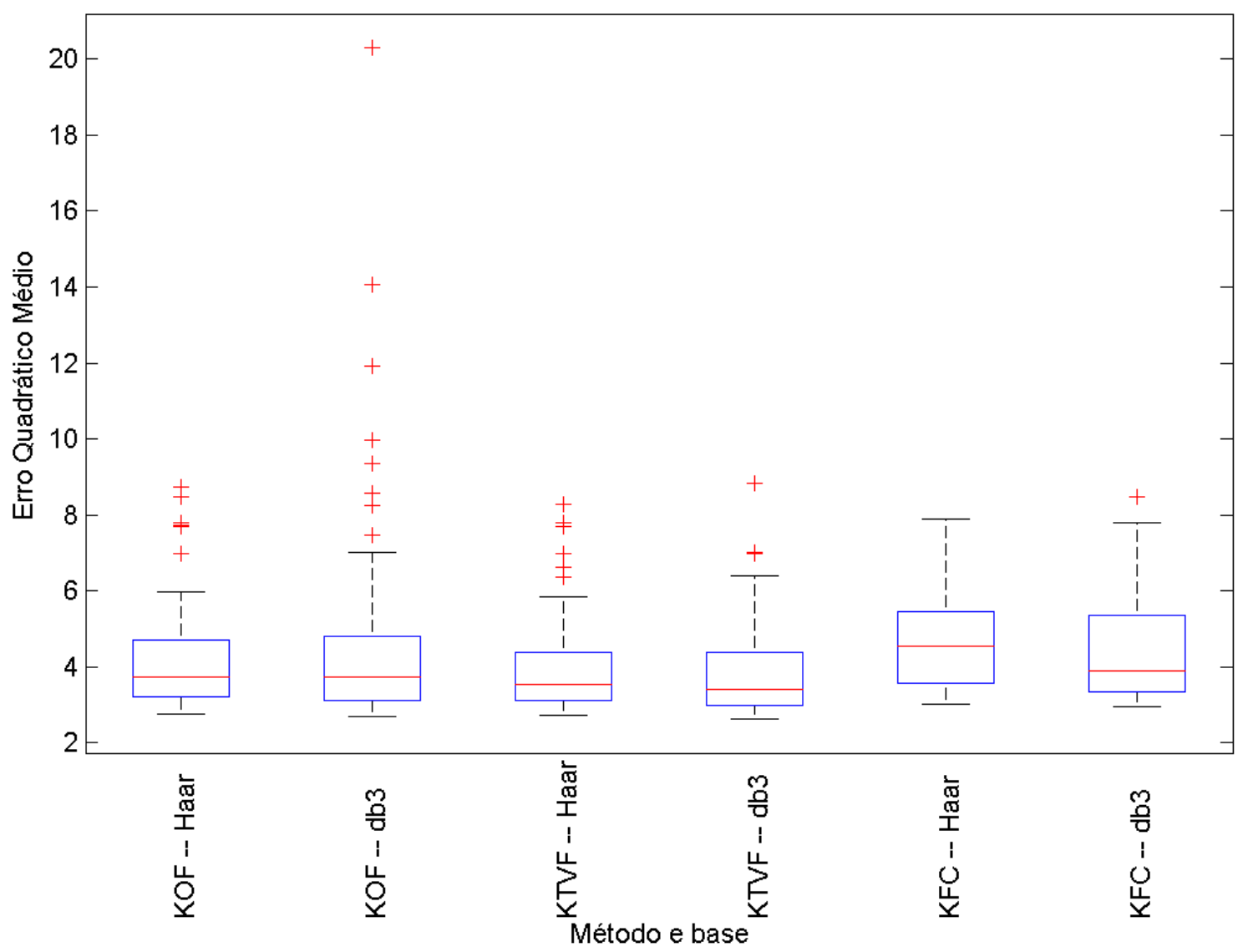

Abreviações: KOF - Krigagem Ordinária Funcional; KTVF - Krigagem Tempo-Variante Funcional; KFC - Krigagem Funcional por Campo; db3 - ondaleta Daubechies com $N=3$.

Figura 5.3: Boxplot de EQM para todas as 82 estações em escala logarítmica. 


\subsection{Material Particulado Inalável - MP10}

Poluição por partículas (também chamada de material particulado ou, brevemente, MP) é o termo para uma mistura de partículas sólidas e gotículas encontradas no ar. Algumas partículas, tais como poeira, sujeira, fuligem ou fumaça, são grandes e escuras o suficiente para serem vistas a olho nu. Outras são tão pequenas que apenas podem ser detectadas usando um microscópio eletrônico. Poluição por partículas é composta por "partículas grossas inaláveis” com diâmetro maior que $2.5 \mu \mathrm{m}$ e menor que $10 \mu \mathrm{m}^{1} \mathrm{e}$ "partículas finas" com diâmetro menor que $2.5 \mu \mathrm{m}$. Algumas partículas, conhecidas como partículas primárias, são emitidas diretamente de uma fonte, como construções civis, estradas não pavimentadas, chaminés de fábricas ou incêndios. Outras são resultados de reações complexas de químicos na atmosfera tais como dióxido sulfúrico e óxido de nitrogênio que são emitidos de usinas térmicas movidas a combustíveis fósseis, indústrias e automóveis ${ }^{2}$. A concentração de microgramas "Material Particulado Grosso" por $m^{3}$, referenciada como MP10, é um índice fortemente relacionado com doenças respiratórias, problemas cardiovasculares e taxa de mortalidade (vide, por exemplo, Ostro et al., 1999; Pope III e Dockery, 2006; Pope III et al., 1991; Schwartz et al., 1993; Seaton et al., 1995, para estudos sobre os efeitos da concentração de material particulado na saúde) e, consequentemente, seu monitoramento e análise são indispensáveis. Nessa Seção, usamos o conjunto de dados composto por medições de MP10 realizadas pela CETESB (Companhia Ambiental do Estado de São Paulo) em 14 localidades listadas na Tabela C.1 do Apêndice C no ano 2014 para interpolar a concentração de microgramas de material particulado grosso por metro cúbico em um local não monitorado pela CETESB usando os três métodos de Krigagem para curvas introduzidos no Capítulo 3: Krigagem Ordinária Funcional, Krigagem Tempo-Variante Funcional e Krigagem Funcional por Campo. Esses dados podem ser obtidos pelo endereço http://www.cetesb.sp.gov.br/.

Para avaliar a performance dos métodos de Krigagem para o índice MP10, escolhemos a estação Parque Dom Pedro II da CETESB identificada pelo ID 72 na Figura 5.4 (quadrado vermelho) e estimamos o índice MP10 usando todas as outras estações destacadas no mapa (quadrados amarelos). Temos 14 estação em nosso conjunto de dados e, consequentemente, usamos 13 estações

\footnotetext{
${ }^{1}$ A sigla $\mu m$ se refere a micrômetro.

${ }^{2}$ Para maiores informações sobre poluição por partícula, visite a página oficial da agência americana United States Environmental Protection Agency http://www3.epa.gov/airquality/particlepollution/basic.html.
} 


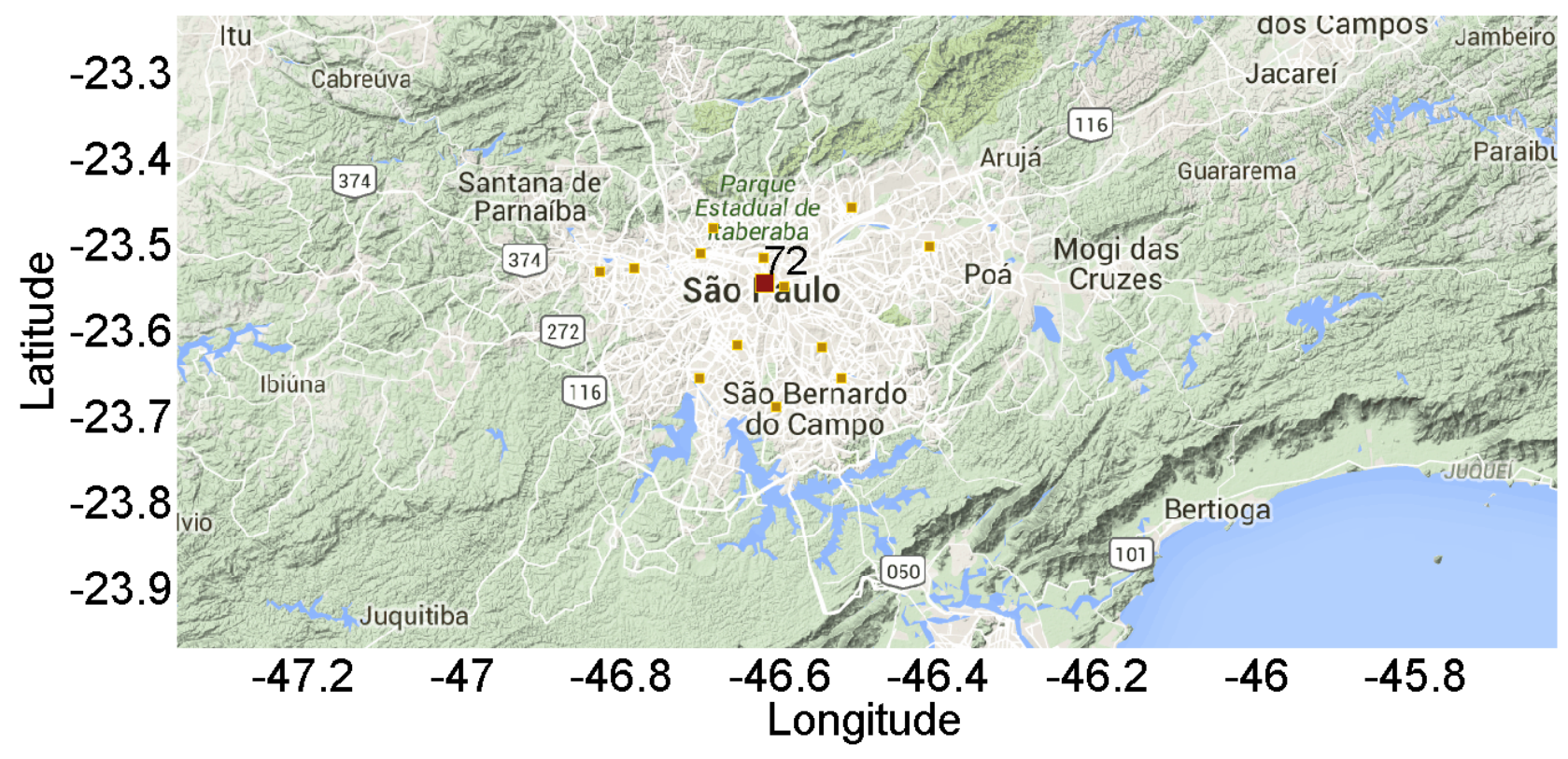

Figura 5.4: Estações meteorológicas na Região Metropolitana de São Paulo.

para estimar a curva de MP10 no ano 2014 na estação Parque Dom Pedro II. Nas Figuras 5.5(a) e 5.5(b), mostramos o ajuste da curva pelo modelo de Krigagem Ordinária Funcional, Krigagem Tempo-Variante Funcional e Krigagem Funcional por Campo. Diferentemente dos dados de temperatura média do Canadá, visualmente os três métodos aparentam ter um desempenho parecido. Contudo, ao analisarmos a Tabela 5.4 e a Figura 5.6, em que apresentamos estatísticas descritivas e o Boxplot em escala logarítmica do $E Q M$ calculado para todas estação, notamos que a Krigagem Funcional por Campo é o método de interpolação espacial de curvas com menor Erro Quadrático Médio, em concordância com o estudo de simulação co Capítulo 4.

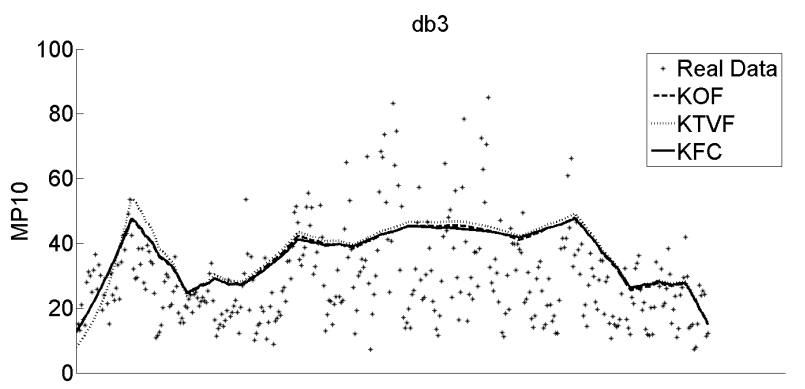

(a) $d b 3$

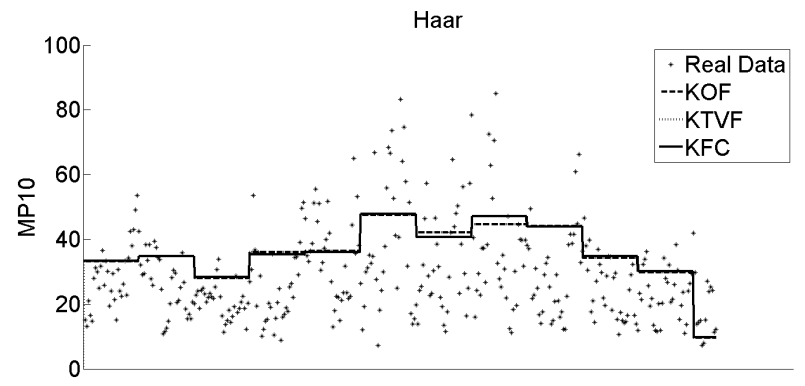

(b) Haar

Abreviações: KOF - Krigagem Ordinária Funcional; KTVF - Krigagem Tempo-Variante Funcional; KFC - Krigagem Funcional por Campo; db3 - ondaleta Daubechies com $N=3$.

Figura 5.5: Curvas ajustadas pelos métodos de interpolação espacial para curvas, Krigagem Ordinária Funcional (KOF), Krigagem Tempo-Variante Funcional e Krigagem Funcional por Campo, usando ondaletas Haar e Daubechies com $N=3$. 


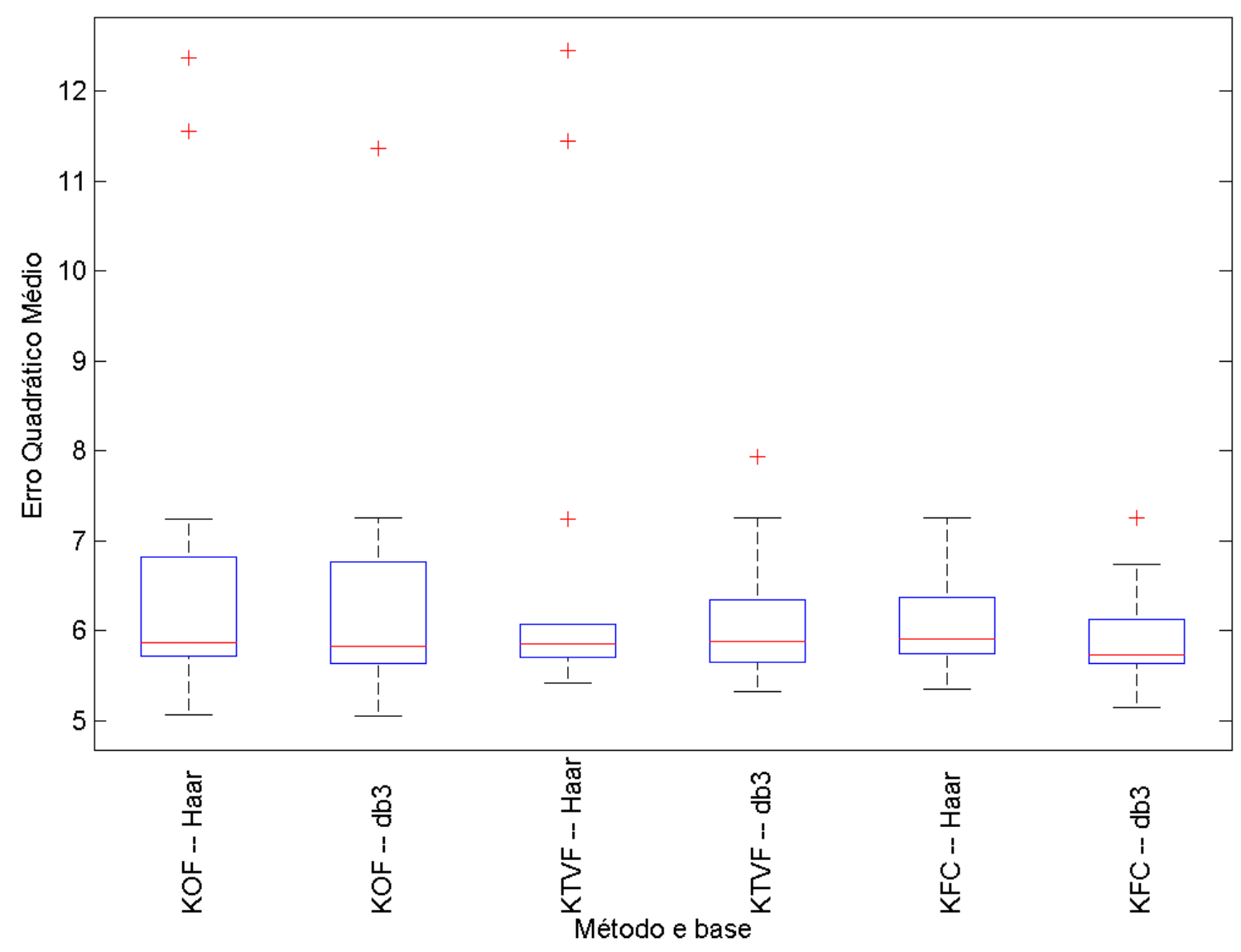

Abreviações: KOF - Krigagem Ordinária Funcional; KTVF - Krigagem Tempo-Variante Funcional; KFC - Krigagem Funcional por Campo; db3 - ondaleta Daubechies com $N=3$.

Figura 5.6: Boxplot de EQM para todas as 14 estações em escala logarítmica. 
Tabela 5.3: Erro Quadrático Médio para a estação Parque Dom Pedro II.

\begin{tabular}{l|cc|cc}
\hline Método & Ondaleta & EQM & Ondaleta & EQM \\
\hline KOF & & 236.11 & & $\mathbf{2 2 4 . 4 8}$ \\
KTVF & Haar & $\mathbf{2 3 5 . 6 6}$ & db3 & 249.75 \\
KFC & & 238.04 & & 225.19 \\
\hline
\end{tabular}

Abreviações: KOF - Krigagem Ordinária Funcional; KTVF - Krigagem Tempo-Variante Funcional; KFC - Krigagem Funcional por Campo; db3 - ondaleta Daubechies com $N=3$.

Tabela 5.4: Sumário de EQM para todas estações.

\begin{tabular}{l|cccccccc}
\hline Método & Mínimo & 1-Qua & Média & Mediana & 3-Qua & Máximo & DP & Soma \\
\hline KFC - db3 & 172.17 & 279.04 & $\mathbf{4 3 0 . 4 5}$ & $\mathbf{3 1 0 . 3 2}$ & 454.55 & 1406.20 & 323.20 & 6026.35 \\
KFC - Haar & 211.56 & 312.56 & 525.06 & 369.69 & 584.09 & 1423.64 & 386.25 & 7350.78 \\
KTVF - db3 & 204.59 & 283.30 & 647.73 & 356.03 & 564.63 & 2796.38 & 704.11 & 9068.28 \\
KOF - db3 & 155.46 & 280.56 & 6631.50 & 340.32 & 870.12 & $8.63 \mathrm{E}+04$ & $2.29 \mathrm{E}+04$ & $9.28 \mathrm{E}+04$ \\
KOF - Haar & 158.35 & 303.48 & $2.47 \mathrm{E}+04$ & 352.88 & 918.39 & $2.36 \mathrm{E}+05$ & $6.67 \mathrm{E}+04$ & $3.45 \mathrm{E}+05$ \\
KTVF - Haar & 224.35 & 300.57 & $2.54 \mathrm{E}+04$ & 349.98 & 430.48 & $2.57 \mathrm{E}+05$ & $7.11 \mathrm{E}+04$ & $3.55 \mathrm{E}+05$ \\
\hline Abreviaçoes: KOF - Krigagem Ordinária Funcional; KTVF - Krigagem Tempo-Variante Funcional; KFC \\
- Krigagem Funcional por Campo; db3 - ondaleta Daubechies com $N=3$.
\end{tabular}




\section{Capítulo 6}

\section{Estudos Futuros}

Desde os trabalhos iniciais Henao (2009), Henao et al. (2010) e Henao et al. (2011), várias melhorias em métodos de krigagem para Dados Funcionais foram sugeridas, contudo ainda existem lacunas que acreditamos que precisam ser superadas e que são descritas a seguir.

\section{Propriedades Assintóticas}

Devido ao caráter recente e inovador dos métodos de krigagem para Dados Funcionais descritos no Capítulo 3 há apenas uma checagem via simulação das propriedades assintóticas, principalmente a consistência. Ou seja, é necessário demonstrar que, sob a condição de estacionaridade fraca pontual e isotropia ${ }^{1}$, as três abordagens de krigagem propostas nesse trabalho são, por exemplo, consistentes.

\section{Abordagem Bayesiana}

Uma possibilidade não explorada nessa tese é o uso da abordagem Bayesiana. Em Geoestatística, há alguns trabalhos usando abordagem Bayesiana. Omre e Halvorsen (1989) apresentaram os métodos de Krigagem Ordinária e Krigagem Universal no contexto de uma abordagem Bayesiana. Pilz e Spöck (2008) propuseram um método de Krigagem denominado Krigagem Trans-Gaussiana Bayesiana para quando a suposição de que temos um campo aleatório Gaussiano e estrutura de covariância conhecida não é razoável. Diggle e Ribeiro Jr (2002) adotaram o paradigma Bayesiano

\footnotetext{
${ }^{1}$ Vide a introdução do Capítulo 3 para a definição de estacionaridade fraca pontual e isotropia para uma amostra funcional georeferenciada.
} 
de estimação para derivar distribuições preditivas condicionais nos dados e apresentaram um algoritmo para amostrar dessas distribuições preditivas. Tais amostras podem ser usadas para predizer valores da variável sob estudo ou de qualquer função linear ou não linear como, por exemplo, a probabilidade dessa variável exceder um limite. De Oliveira et al. (1997) estenderam a teoria de krigagem do contexto de campo aleatório Gaussiano para campo aleatório transformado, em que uma transformação nos dados é realizada para moderar o afastamento da normalidade e a transformação pertence a uma família paramétrica de transformações. Fuentes (2001) introduziu um novo método de interpolação espacial para processos não estacionários. Nesse método, o campo é representado localmente como um campo aleatório isotrópico e estacionário, mas os parâmetros podem variar no espaço e uma abordagem Bayesiana é proposta para incorporar a incerteza na estimação dos parâmetros da covariância. Na ausência de um amplo estudo comparando a performance dos métodos de Krigagem, Moyeed e Papritz (2002) estudaram várias abordagens de comparação, incluindo abordagens Bayesianas, em um conjunto de dados de concentração de cobalto (Co) e cobre (Cu) em 2649 localidades em Scottish Border, região administrativa da Escócia - Reino Unido. Brown et al. (1994) desenvolveram um método de Krigagem para campos aleatórios multivariados usando abordagem Bayesiana, em que obtiveram a distribuição a posteriori para vetores não observados e para os parâmetros do modelo. A ideia seria adaptar esses métodos de Krigagem para Dados Funcionais, de maneira análoga aos trabalhos de Ramón G. Heano no final da década de 2000 em Henao (2009); Henao et al. (2010, 2011).

\section{Outros Modelos}

Um terceiro modelo de Krigagem para Dados Funcionais foi proposto no final da década 2000 em Henao (2009) e em Nerini et al. (2010) e ele não foi estudado nem implementado usando ondaletas nem sua performance foi analisada. Mais precisamente, dado uma amostra funcional $\chi_{\boldsymbol{s}_{1}}(t), \ldots, \chi_{\boldsymbol{s}_{n}}(t)$ isotrópica e pontualmente fracamente estacionária conforme descrito na introdução do Capítulo 3, uma estimativa para a curva $\chi_{s_{0}}(t)$ em um ponto não monitorado $\boldsymbol{s}_{0}$ é a solução do problema de otimização não linear descrito por 


$$
\hat{\chi}_{\boldsymbol{s}_{0}}(t)=\sum_{i=1}^{n} \int_{0}^{1} \lambda_{i}(t, u) \chi_{\boldsymbol{s}_{i}}(u) d u, \quad \forall t \in[0,1] \text { sujeito a } \mathrm{E}\left\{\hat{\chi}_{\boldsymbol{s}_{0}}(t)\right\}=\chi_{\boldsymbol{s}_{0}}(t), \quad \forall t \in[0,1]
$$

em que $\lambda(.,):.[0,1] \rightarrow \mathbb{R}$ e $\chi_{\boldsymbol{s}_{i}}:[0,1] \rightarrow \mathbb{R}$ precisam ser aproximados usando uma base de funções e B-Splines foram usadas. Além disso, notamos que nesse projeto optamos pelo uso de estimadores não paramétricos para o semivariograma, mas uma alternativa possível é usar estimadores paramétricos conforme descrito em Banerjee et al. (2014); Genton e Kleiber (2015). Adicionalmente, podemos substituir a aproximação numérica de integrais por retângulos descrita na Seção 3.2 por métodos mais eficientes e precisos, como, por exemplo, uma aproximação por trapézios.

\section{Implementação}

Um dos subprodutos desse projeto de doutorado é a Toolbox do MATLAB denominada krigingFDA. Contudo, para o seu uso além de ter uma licença válida do MATLAB, é preciso ter as seguintes Toolbox instaladas

\section{- Mapping Toolbox}

\section{- Optimization Toolbox}

- Statistics and Machine Learning Toolbox

\section{- Econometrics Toolbox}

o que pode invializar o uso das implementações descritas no Apêndice A para pequenas empresas ou centros de pesquisa emergentes. Neste contexto, uma "tradução" da Toolbox krigingFDA para plataformas livres como Octave (John W. Eaton e Wehbring, 2015) e R R Core Team (2015) é desejável. O Octave tem uma sintaxe próxima do MATLAB, sendo considerada uma versão livre do mesmo, mas seu uso é pouco disseminado entre a comunidade estatística. O R, por outro lado, tem uma sintaxe bastante distante do MATLAB, mas seu uso é muito mais disseminado para análises estatísticas. 


\section{Amostras Funcionais de Grande Dimensão}

Um ponto que devemos destacar é que todos métodos de Krigagem para Dados Fucionais descritos no Capítulo 3 são combinações lineares de curvas ou de coeficientes da projeção das curvas em um espaço de aproximação. E quando o número de elementos da amostra funcional cresce, o número de parâmetros que precisamos estimar também aumenta. Na verdade, métodos de krigagem envolvem inversão de matrizes cuja complexidade numérica é da ordem de pelo menos $O\left(n^{3}\right)$. Não fomos capazes de encontrar soluções na literatura para esse tema no contexto de Dados Funcionais, mas em Geoestatística existem algumas propostas e Sun et al. (2012) faz uma revisão dos métodos de Geoestatística quando a amostra é grande. Merecem destaque o trabalho de Banerjee et al. (2008), denominado processo preditivo espacial, em que o processo espacial original é projetado em um subespaço gerado pelas realizações do processo original em conjunto pré-especificado de localidades e a proposta de Cressie e Johannesson (2008) em que os autores consideraram uma classe de matrizes de variância-covariância $\Sigma$ de ordem $n \times n$ tal que a inversa $\Sigma^{-1}$ pode ser obtida invertendo matrizes de ordem $r \times r \operatorname{com} r$ fixo e $r<n$, e, consequentemente, a complexidade numérica dos métodos de Krigagem decai de $O\left(n^{3}\right)$ para $O\left(n r^{2}\right)$, além disso essa abordagem é capaz de tratar simultanemanente com o tamanho da amostra e a heterogeneidade espacial. 


\section{Apêndice A}

\section{Documentação da Toolbox krigingFDA do MATLAB}

Neste apêndice, documentamos a Toolbox do MATLAB desenvolvida durante este projeto e denominada krigingFDA.

A Toolbox krigingFDA tem quatorze funções:

FieldKriging_Haar Esta função implementa o método Krigagem Funcional por Campo usando ondaleta Haar.

chi_Haar Esta função calcula a curva estimada usando ondaleta Haar.

FieldHatchi_Haar Esta função calcula a curva $\chi_{\boldsymbol{s}_{0}}(t)$ no instante $t$ no ponto não monitorado $\boldsymbol{s}_{0}$ para a ondaleta Haar no modelo de Krigagem Funcioanl por Campo.

FieldKriging_dbN Esta função implementa o médodo de Krigagem Funcional por Campo usando ondaleta da família Daubechies.

chi_dbN Esta função calcula curva estimada usando ondaleta Daubechies

FieldHatchi_dbN Esta função calcula a curva $\chi_{\boldsymbol{s}_{0}}(t)$ no instante $t$ no ponto não monitorado $s_{0}$ para a família Daubechies no modelo de Krigagem Funcional por Campo.

FunctionalKriging Esta função é uma implementação para o método Krigagem Ordinária Funcional usando ondaletas Haar.

PointwiseFuncKriging Implementação do método de Krigagem Tempo-Variante Funcional usando ondaletas Haar.

coef_Haar Calcula os coeficientes de uma curva suavizada usando ondaletas Haar

coef_dbN Calcula os coeficientes de uma curva suavizada usando ondaletas Daubechies

FuncKriging_dbN Implementação do método de Krigagem Ordinária Funcional usando ondaletas Daubechies.

hatchi_s0 Calcula a curva estimada $\chi_{\boldsymbol{s}_{0}}(t)$ no instante $t$ para o modelo de Krigagem Ordinária Funcional para ondaletas Daubechies.

PointwiseFuncKriging_dbN Implementação do método de Krigagem Tempo-Variante Funcional para ondaletas Daubechies. 
hatchi Calcula a curva estimada $\chi_{\boldsymbol{s}_{0}}(t)$ no instante $t$ para o modelo de Krigagem TempoVariante Funcional para ondaletas Daubechies.

A descrição de cada uma dessas funções é descrita em uma seção organizada em quatro partes: objetivo, sintaxe, descrição e código fonte.

\section{A.1 FieldKriging_Haar}

\section{Objetivo}

Esta função implementa o método descrito na Seção 3.3 em que desejamos encontrar o vetor de coeficientes $\boldsymbol{a}_{0}$ da projeção da curva $\chi_{\boldsymbol{s}_{0}}(t)$ em um ponto não monitorado $\boldsymbol{s}_{0}$ usando ondaletas Haar.

\section{Sintaxe}

[a0, mL, mcoef]=FieldKriging_Haar(mdata, lat, long, latData, longData, J, tol);

\section{Descrição}

A função FieldKriging_Haar tem sete argumentos de entrada:

i. mdata é um matriz em que a i-ésima coluna é a série temporal $\chi_{\boldsymbol{s}_{i}}\left(t_{1}\right), \ldots, \chi_{\boldsymbol{s}_{i}}\left(t_{n}\right)$ observada da curva $\chi_{s_{i}}(t)$

ii. lat é a latitude do ponto não monitorado $\boldsymbol{s}_{0}$

iii. long é a longitude do ponto não monitorado $s_{0}$

iv. LatData é um vetor coluna contendo as latitudes dos pontos $\boldsymbol{s}_{1}, \ldots, \boldsymbol{s}_{n}$ das curvas em nossa amostra funcional

v. longData é um vetor coluna contendo as longitudes dos pontos $\boldsymbol{s}_{1}, \ldots, \boldsymbol{s}_{n}$ das curvas em nossa amostra funcional

vi. J é o nível de aproximação na análise de multirresolução

vii. tol é o nível de tolerância para o cálculo do semivariograma conforme explicado no Capítulo 3. Esse argumento é opcional e seu valor padrão é 0.1

e tem três saídas

a. $\quad$ a 0 é um vetor coluna de coeficientes da projeção da curva $\chi_{\boldsymbol{s}_{0}}(t)$ no ponto não monitorado $\boldsymbol{s}_{0}$ usando método de krigagem introduzido na Seção 3.3

b. mL é um matriz em que a i-ésima coluna contém a diagonal da matriz $\Lambda_{i}$

c. mcoef é uma matriz em que a i-ésima coluna armazena os coeficientes da curva $\chi_{\boldsymbol{s}_{i}}(t)$ no ponto $s_{i}$ 


\section{Código Fonte}

Listing A.1: Arquivo FieldKriging_Haar.m.

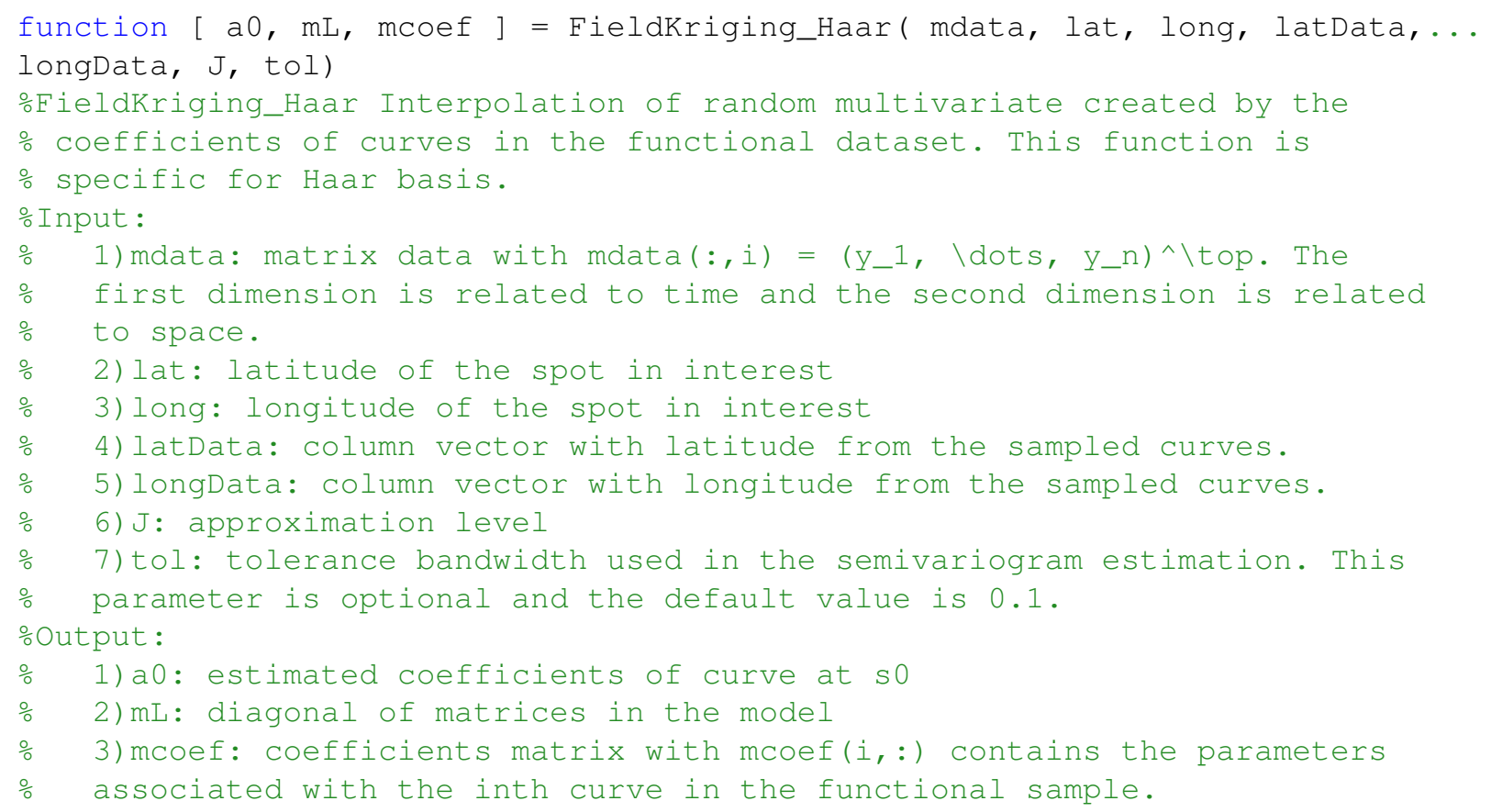




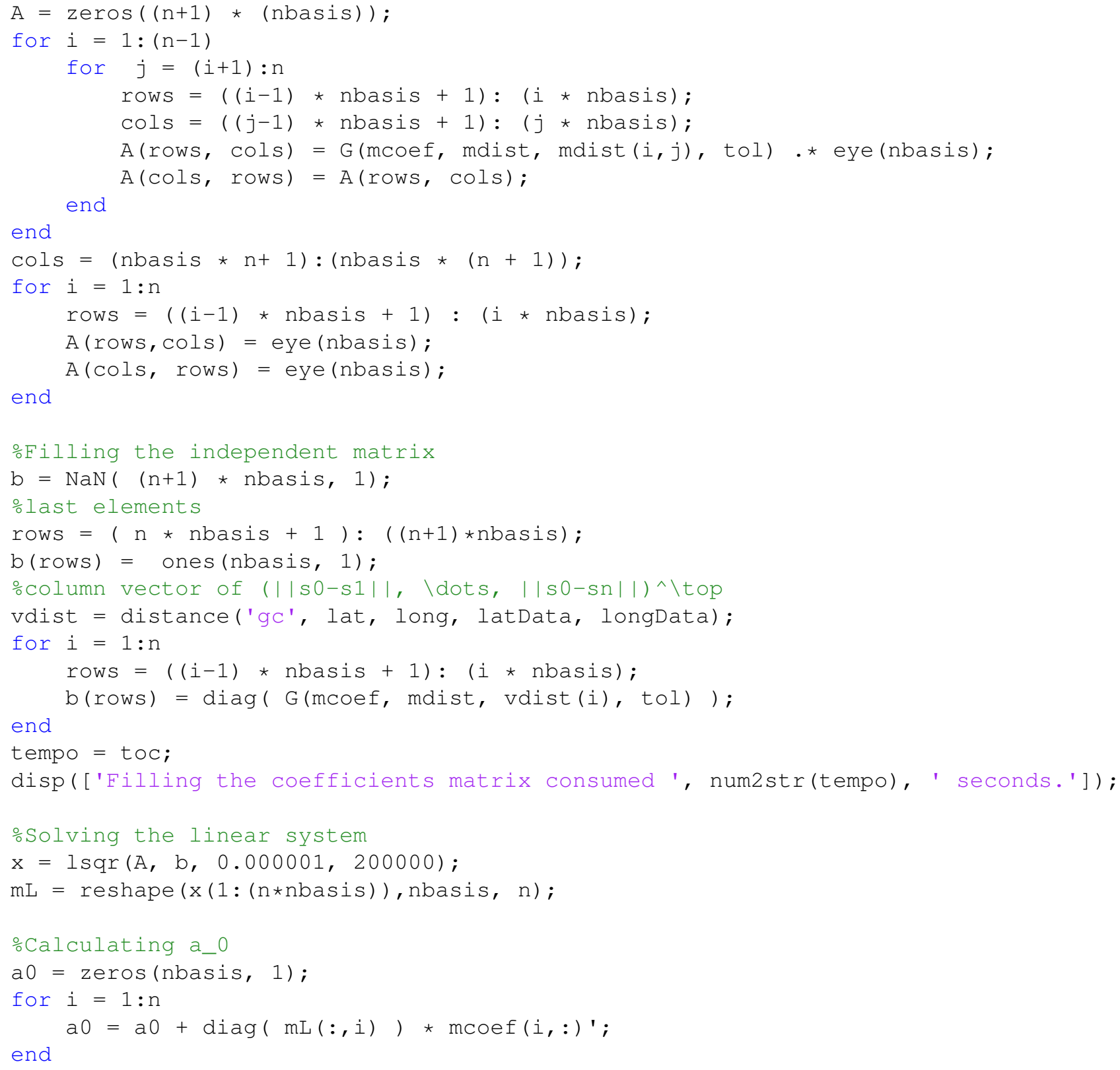

\% 1) mG: estiamted semivariogram at $h$

optional argument. Tolerance bandwidth.

if nargin $==3$

tol $=0.1$;

end 


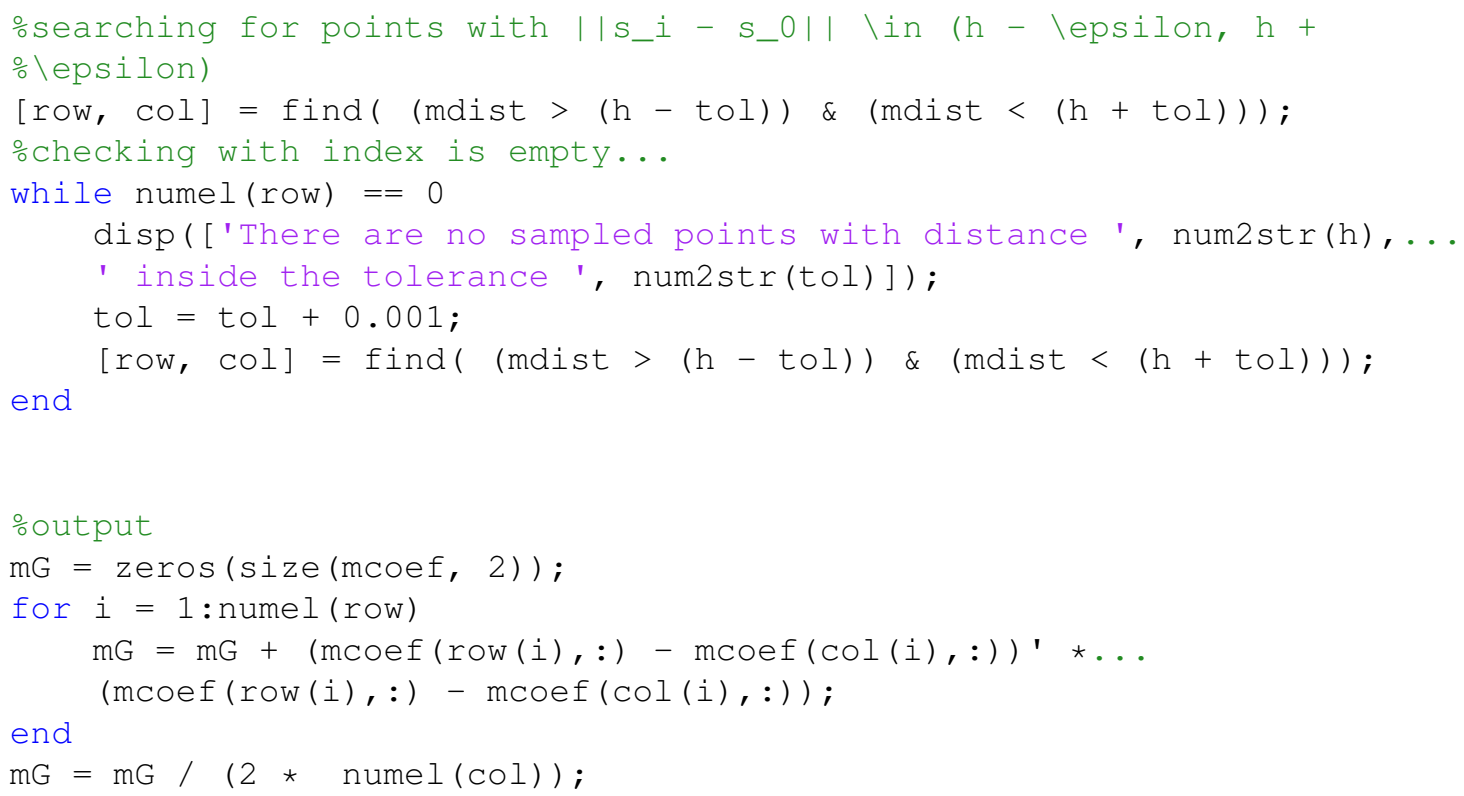

\section{A.2 chi_Haar}

\section{Objetivo}

Esta função calcula o valor da curva estimada usando ondaletas Haar instante $t$ dado os coeficientes calculados pela função coef_Haar.

\section{Sintaxe}

$f=\operatorname{chiHaar}(t, J, c)$

\section{Descrição}

A função chi_Haar tem três argumentos:

i. t é um vetor coluna de instantes para calcularmos o valor da curva

ii. J é o nível de aproximação

iii. c é um vetor coluna contendo os coeficientes estimados usando a função coef_Haar e retorna apenas um vetor coluna contendo os valores de curva $\chi(t)$ nos elementos do vetor coluna t.

\section{Código fonte}




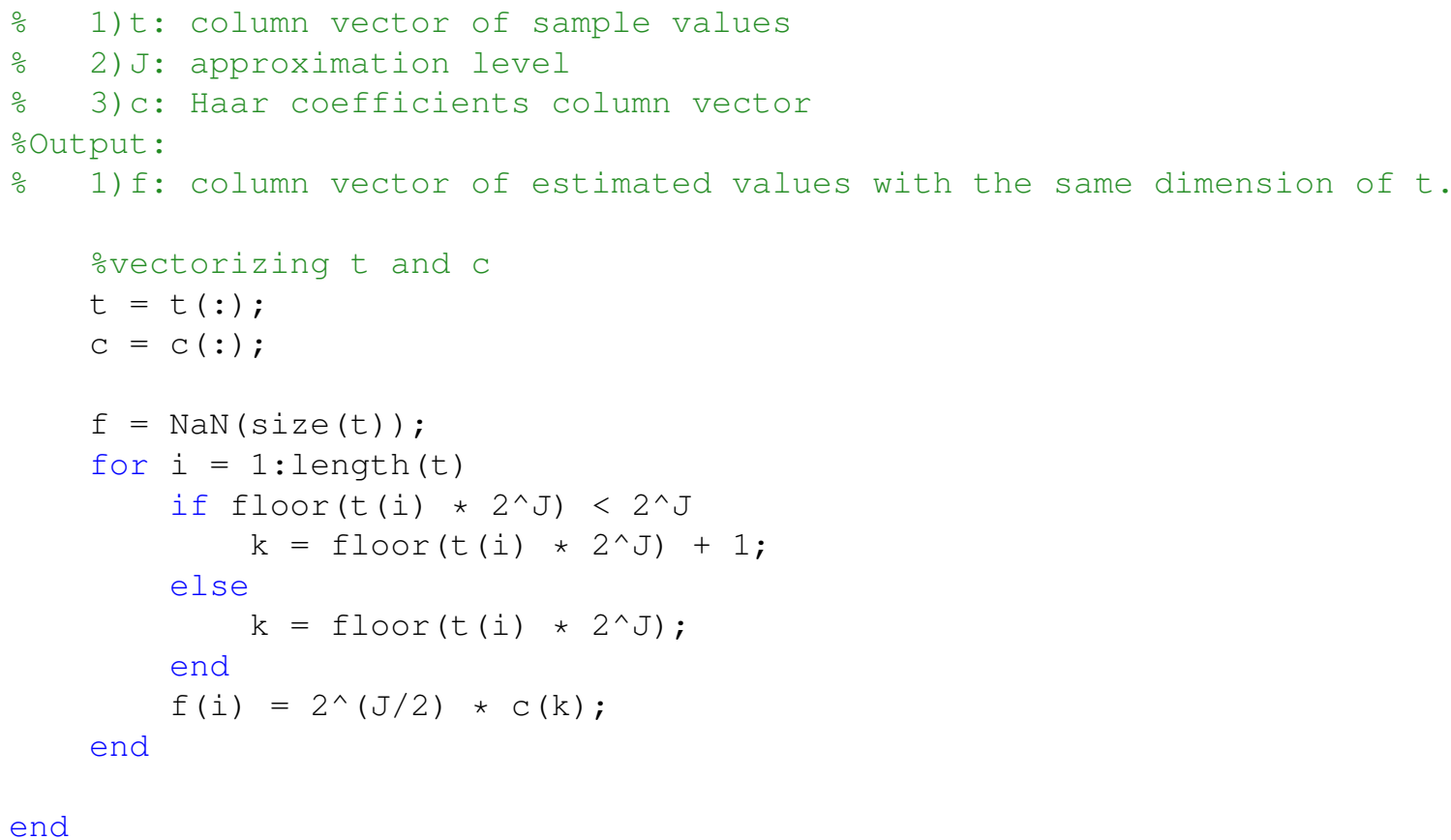

\section{A.3 FieldHatchi_Haar}

\section{Objetivo}

Esta função calcula a curva $\chi_{s_{0}}(t)$ em $t$ no ponto não monitorado $s_{0}$ dado a diagonal das matrizes $\Lambda_{i}, i=1, \ldots, n$ e o coeficientes das curvas $\chi_{\boldsymbol{s}_{i}}(t), i=1, \ldots, n$ usando ondaletas Haar conforme descrito na Seção 3.3.

\section{Sintaxe}

[ $\mathrm{f}]=$ FieldHatchi_Haar $(\mathrm{t}, \mathrm{mL}, \operatorname{mcoef})$;

\section{Descrição}

A função FieldHatchi_Haar tem três argumentos de entrada

i. $t$ é um vetor coluna de pontos em que desejamos calcular o valor estimado da curva $\chi_{s_{0}}(t)$ no ponto $s_{0}$

ii. mL é uma matriz em que a i-ésima coluna contém a diagonal da matriz $\Lambda_{i}$ do modelo de krigagem descrito na Seção 3.3

iii. mcoef é uma matriz em que a i-ésima coluna é preenchida com os coeficientes da curva $\chi_{s_{i}}(t)$

e retorna um vetor coluna $\mathrm{f}=\left(\chi_{\boldsymbol{s}_{0}}\left(t_{1}\right), \ldots, \chi_{\boldsymbol{s}_{0}}\left(t_{m}\right)\right)^{\top}$ com o mesmo número linhas de $\mathrm{t}$.

\section{Código Fonte}


Listing A.2: Arquivo FieldHatchi_Haar.m.

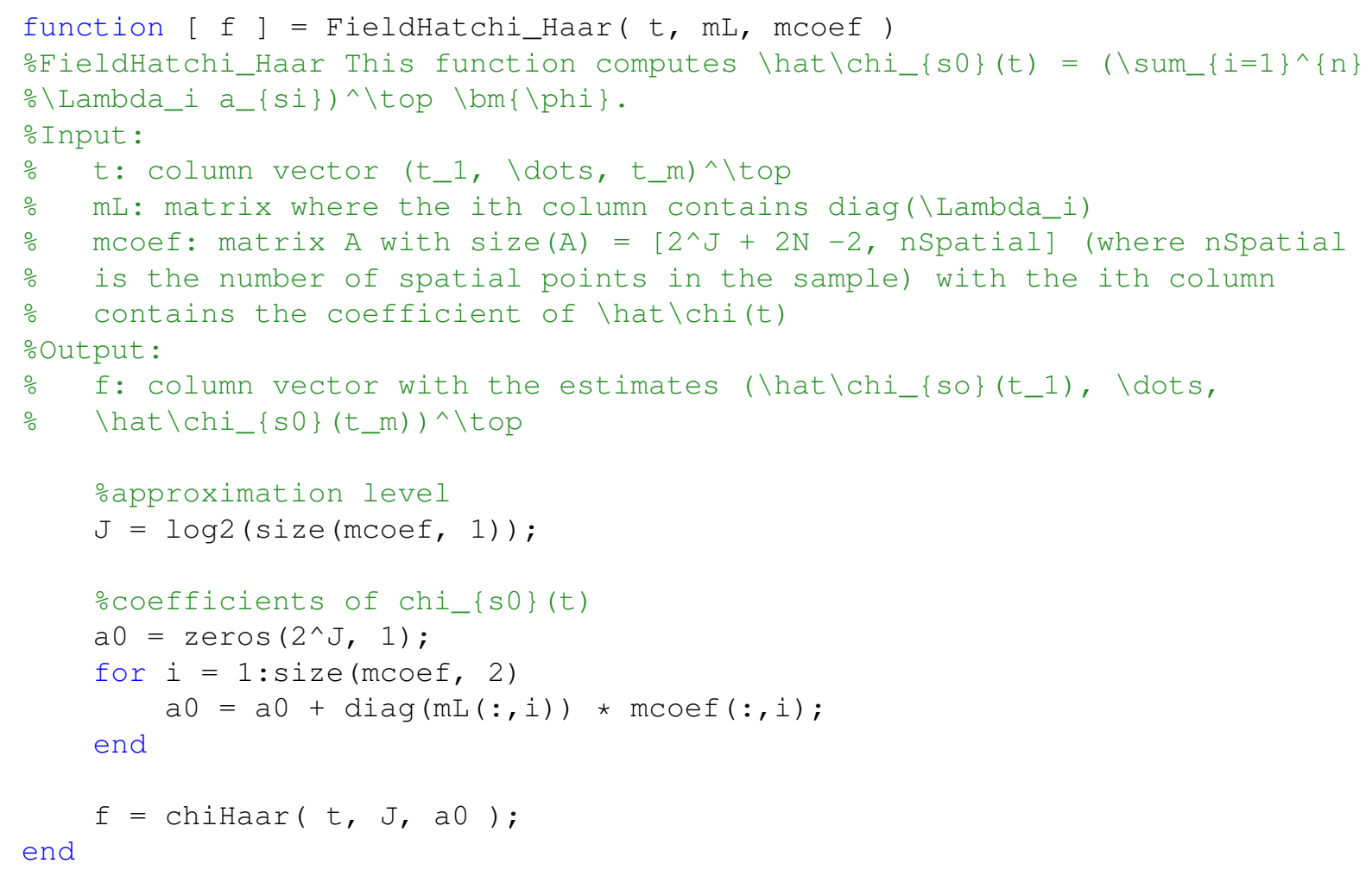

\section{A.4 FieldKriging_dbN}

\section{Objetivo}

Esta função calcula a curva $\chi_{\boldsymbol{s}_{0}}(t)$ em $t$ no ponto não monitorado $\boldsymbol{s}_{0}$ dado a diagonal das matrizes $\Lambda_{i}, i=1, \ldots, n$ e o coeficientes das curvas $\chi_{\boldsymbol{s}_{i}}(t), i=1, \ldots, n$ usando ondaletas Daubechies conforme descrito na Seção 3.3.

\section{Sintaxe}

[a0, mL, mcoef]=FieldKriging_dbN(mdata, lat, long, latData, longData, Iter, J, N, tol);

\section{Descrição}

A função FieldKriging_dbN tem nove argumentos de entrada:

i. mdata é um matriz em que a i-ésima coluna é a série temporal $\chi_{\boldsymbol{s}_{i}}\left(t_{1}\right), \ldots, \chi_{\boldsymbol{s}_{i}}\left(t_{n}\right)$ observada da curva $\chi_{\boldsymbol{s}_{i}}(t)$

ii. lat é a latitude do ponto não monitorado $s_{0}$

iii. long é a longitude do ponto não monitorado $s_{0}$

iv. LatData é um vetor coluna contendo as latitudes dos pontos $\boldsymbol{s}_{1}, \ldots, \boldsymbol{s}_{n}$ das curvas em nossa amostra funcional

v. longData é um vetor coluna contendo as longitudes dos pontos $\boldsymbol{s}_{1}, \ldots, \boldsymbol{s}_{n}$ das curvas em nossa amostra funcional 
vi. Iter é uma número inteiro positivo tal que $\frac{1}{2^{\text {Iter }}}$ precisão numérica usada nas aproximações de integrais envolvendo a função escala

vii. J é o nível de aproximação na análise de multirresolução

viii. N é um número inteiro positivo que indexa a ordem na família Daubechies

ix. tol é o nível de tolerância para o cálculo do semivariograma conforme explicado no Capítulo 3. Esse argumento é opcional e seu valor padrão é 0.1

e retorna três argumentos

a. $a_{0}$ é o vetor colunas contendo os coeficientes estimados da curva $\chi_{s_{0}}(t)$

b. mL é uma matriz em que a i-ésima coluna contém a diagonal principal do matriz $\Theta_{i}$ do modelo Krigagem Funcional por Campo

c. mcoef é uma matriz cuja i-ésima coluna contém os coeficientes da curva $\chi_{\boldsymbol{s}_{i}}(t)$

\section{Código Fonte}

Listing A.3: Arquivo FieldKriging_dbN.m.

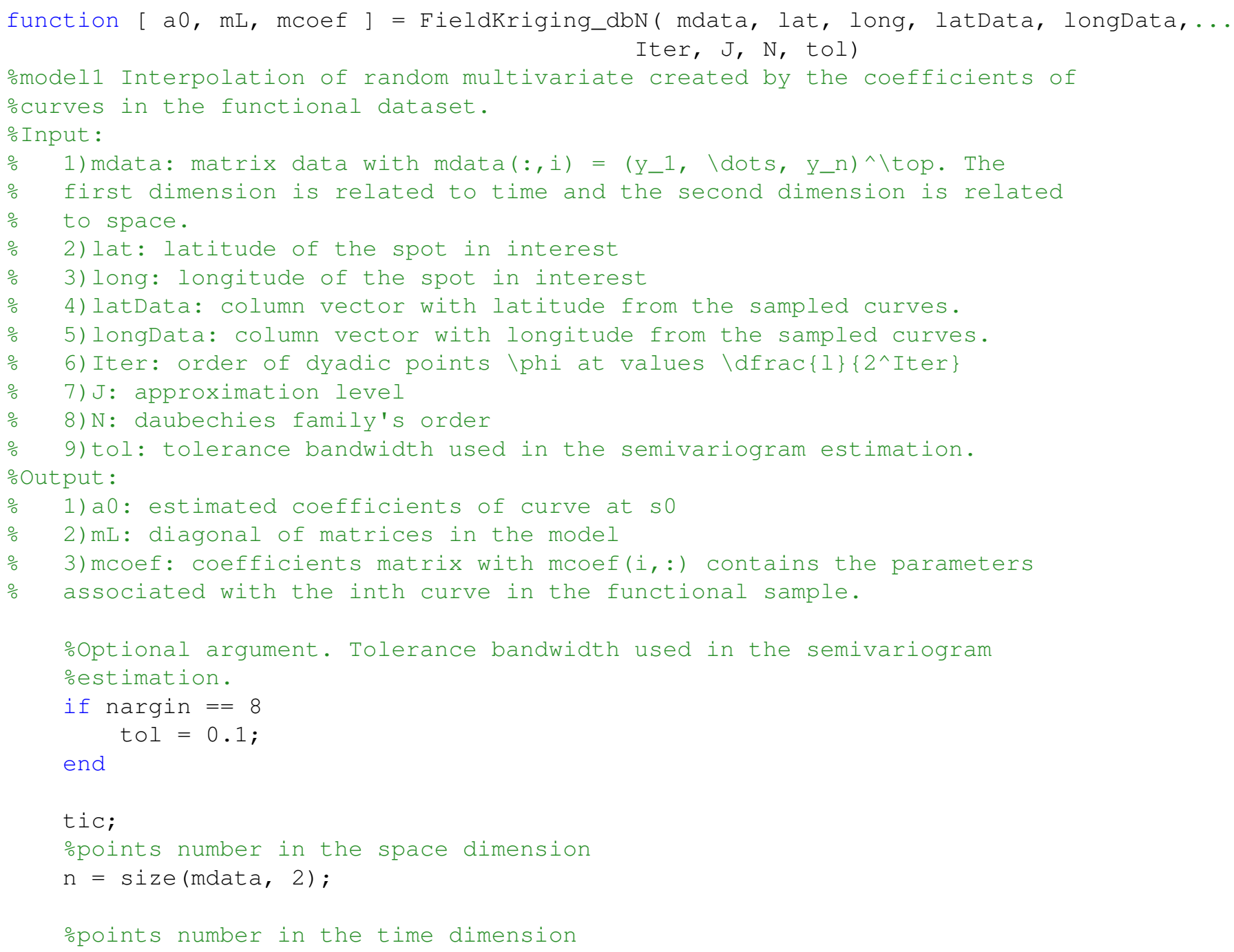




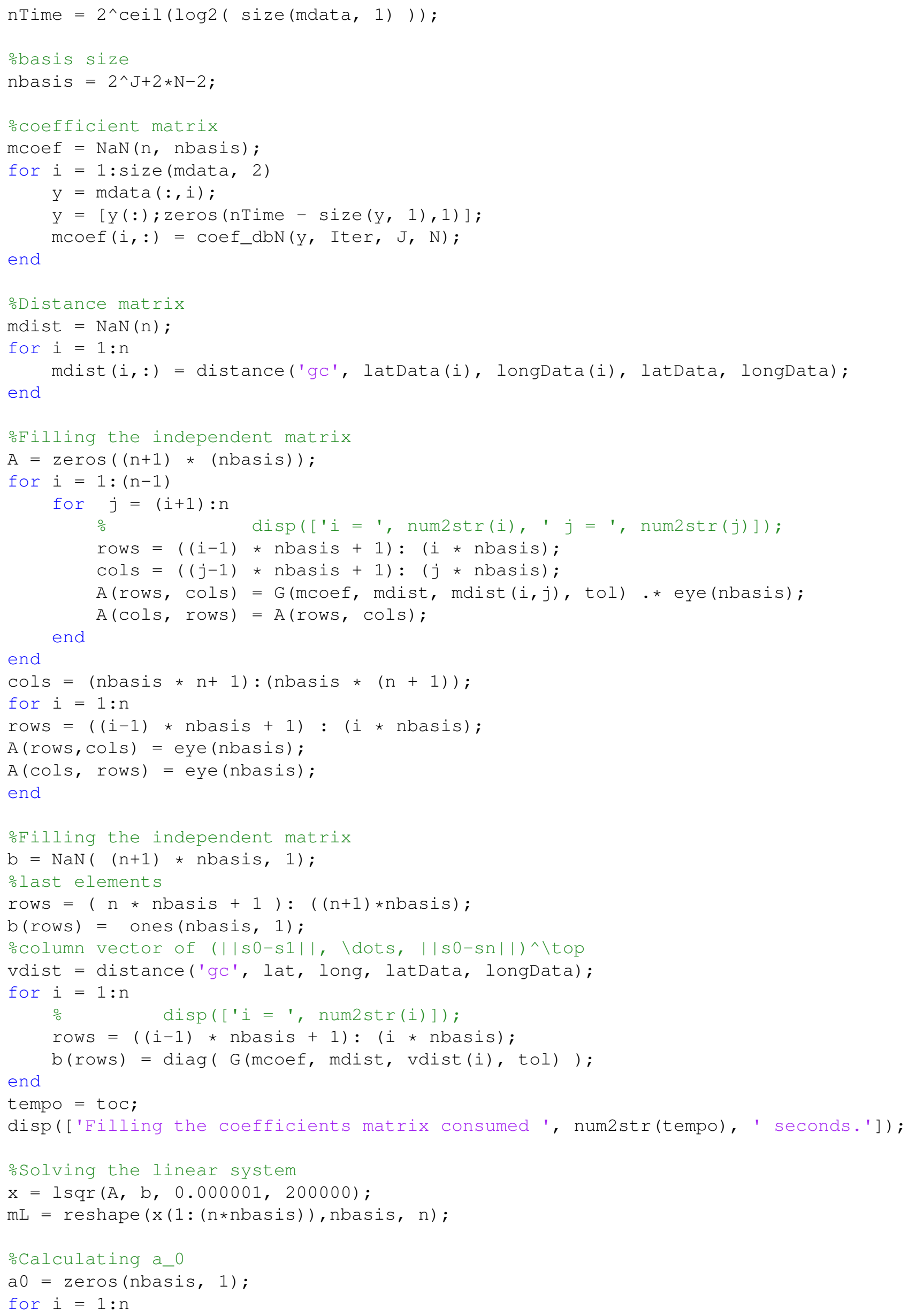




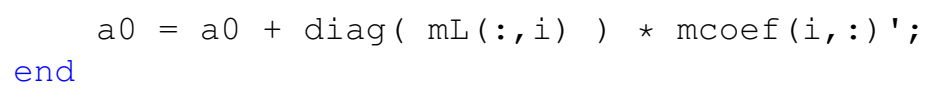

end

function $[\mathrm{mG}]=\mathrm{G}($ mcoef, mdist, $\mathrm{h}$, tol)

oG This function computes the semivariogram for a multivariate random ㄷield.

○Input

\% 1)mcoef: mcoef: coefficients matrix with mcoef(i,:) contains the parameters

o associated with the inth curve in the functional sample.

\% 2) mdist: distance matris with $\operatorname{moist}(i, j)=|| s_{-} i-s_{-} j||$

\% 3) h: distance target

\% 4)tol: tolerance bandwidth. Optional argument and default value is $\circ 0.01$.

Output:

\% 1)mG: estiamted semivariogram at $h$

optional argument. Tolerance bandwidth.

if nargin $==3$

tol $=0.1$;

end

osearching for points with ||s_i - s_0|| \in (h - lepsilon, $\mathrm{h}+$

ㄴopilon)

$[$ row, col $]=$ find $($ mdist $>(h-t o l)) \&(\operatorname{mdist}<(h+$ tol $)))$;

ochecking with index is empty...

while numel(row) $==0$

disp(['There are no sampled points with distance ', num2str(h),...

' inside the tolerance ', num2str(tol)]);

tol $=$ tol +0.001 ;

$[$ row, col $]=$ find $($ mdist $>(h-t o l)) \&($ mdist $<(h+$ tol $)))$;

end

\%output

$\mathrm{mG}=\operatorname{zeros}($ size (mcoef, 2)) ;

for $i=1$ :numel (row)

$\mathrm{mG}=\mathrm{mG}+(\operatorname{mcoef}(\operatorname{row}(i),:)-\operatorname{mcoef}(\operatorname{col}(i),:))^{\prime} \star \ldots$

(mcoef(row $(i),:)-\operatorname{mcoef}(\operatorname{col}(i),:))$;

end

$\mathrm{mG}=\mathrm{mG} /(2 \star$ numel $(\operatorname{col})) ;$

end

\section{A.5 chi_dbN}

\section{Objetivo}

Esta função calcula o valor da curva estimada em um vetor coluna de pontos em $[0,1]$ dado os coeficientes calculados pela função coef_dbN.

\section{Sintaxe}

$\mathrm{f}=$ chi_dbN $(\mathrm{t}$, Iter, J, N, c ) ; 


\section{Descrição -}

A função chi_dbN tem cinco argumentos de entrada

i. $t$ é um vetor coluna de pontos $\left(t_{1}, \ldots, t_{m}\right)^{\top} \operatorname{com} t_{j} \in[0,1]$

ii. Iter é um número inteiro positivo em $\frac{1}{2^{\text {Iter }}}$ é a precisão da aproximação numérica de integrais envolvendo a função escala

iii. J é o nível de aproximação

iv. N é um número inteiro indicando a ordem na família Daubechies

v. c é um vetor coluna contendo os coeficientes estimados pela função coef_dbN

e retorna um vetor coluna $f=\left(\chi\left(t_{1}\right), \ldots, \chi\left(t_{m}\right)\right)^{\top}$ de mesma dimensão do vetor coluna $t$.

\section{Código Fonte}

Listing A.4: Arquivo chi_dbN.m

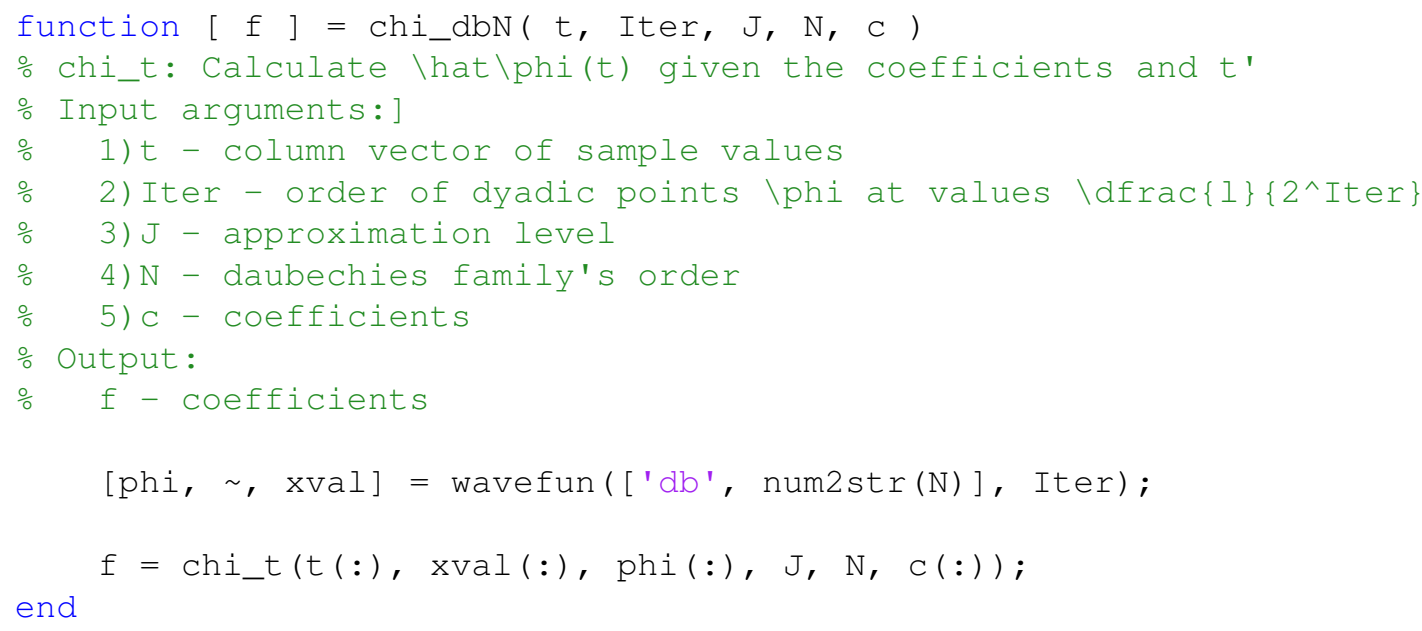

Observe que a função chi_dbN descrita em Listing A.4 usa o MEX file imprimido em Listing A.5. Vide o site oficial do MATLAB para informação sobre como compilar MEX file em cada sistema operacional.

Listing A.5: Arquivo chi_t.c

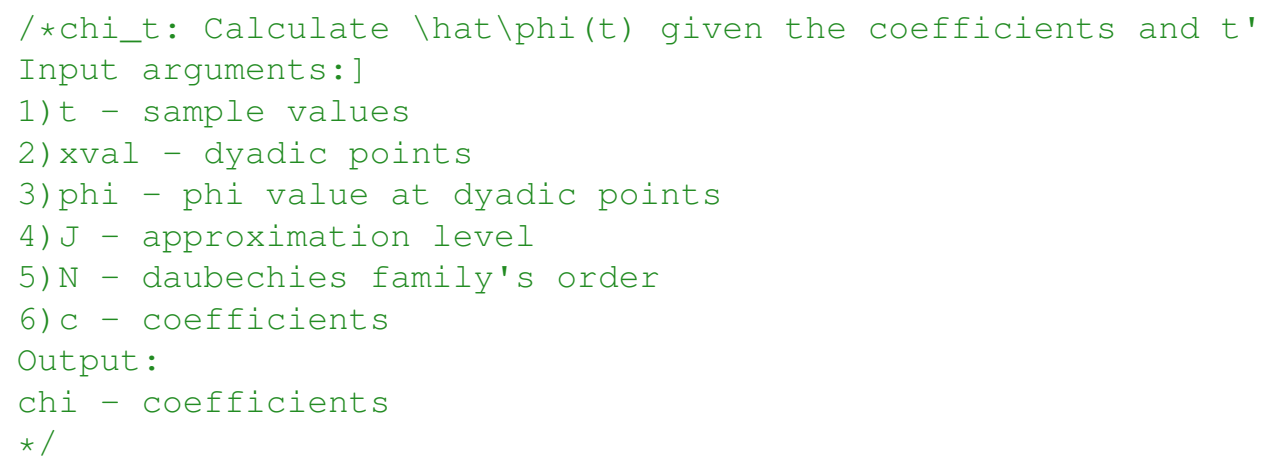




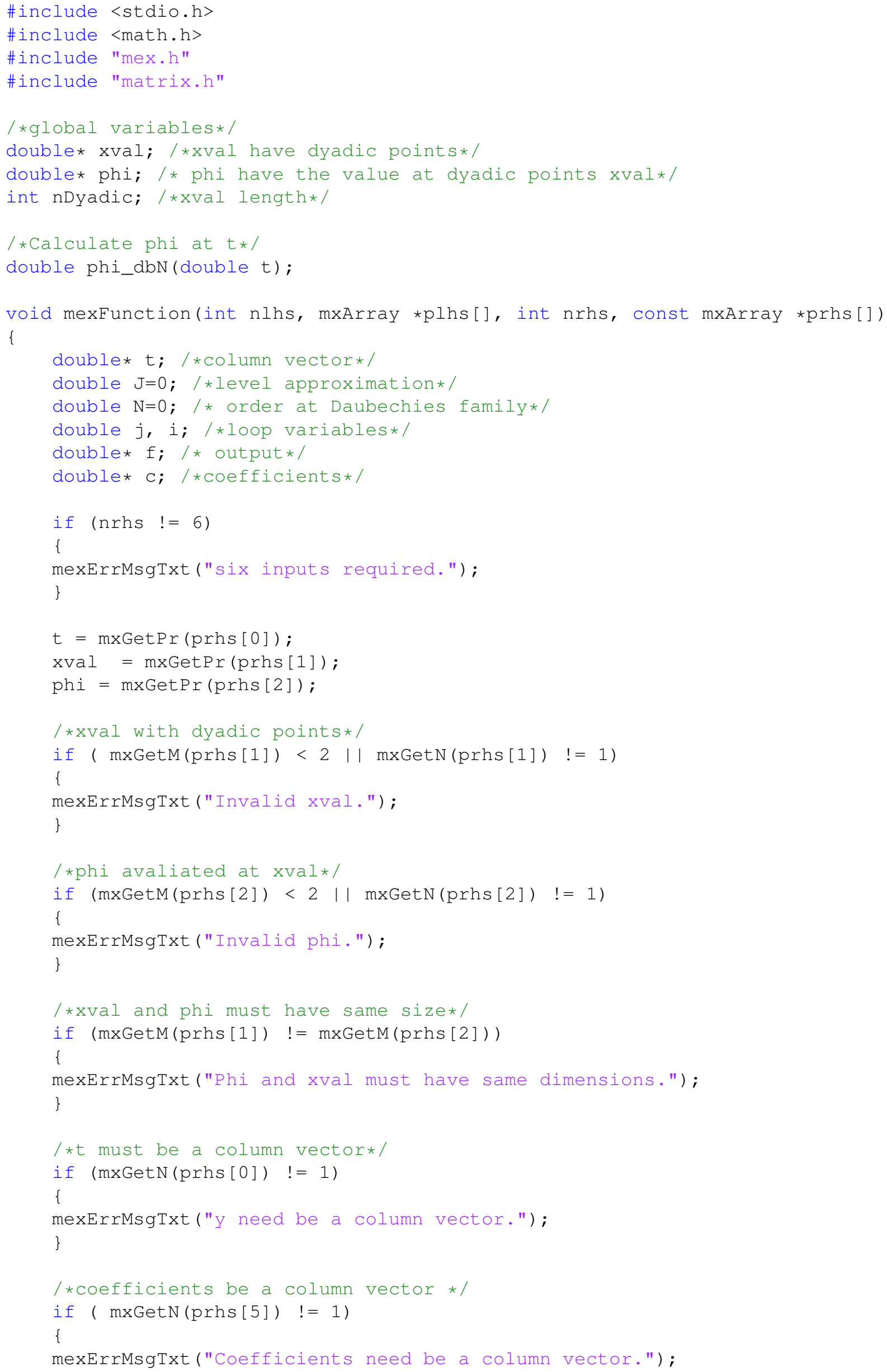




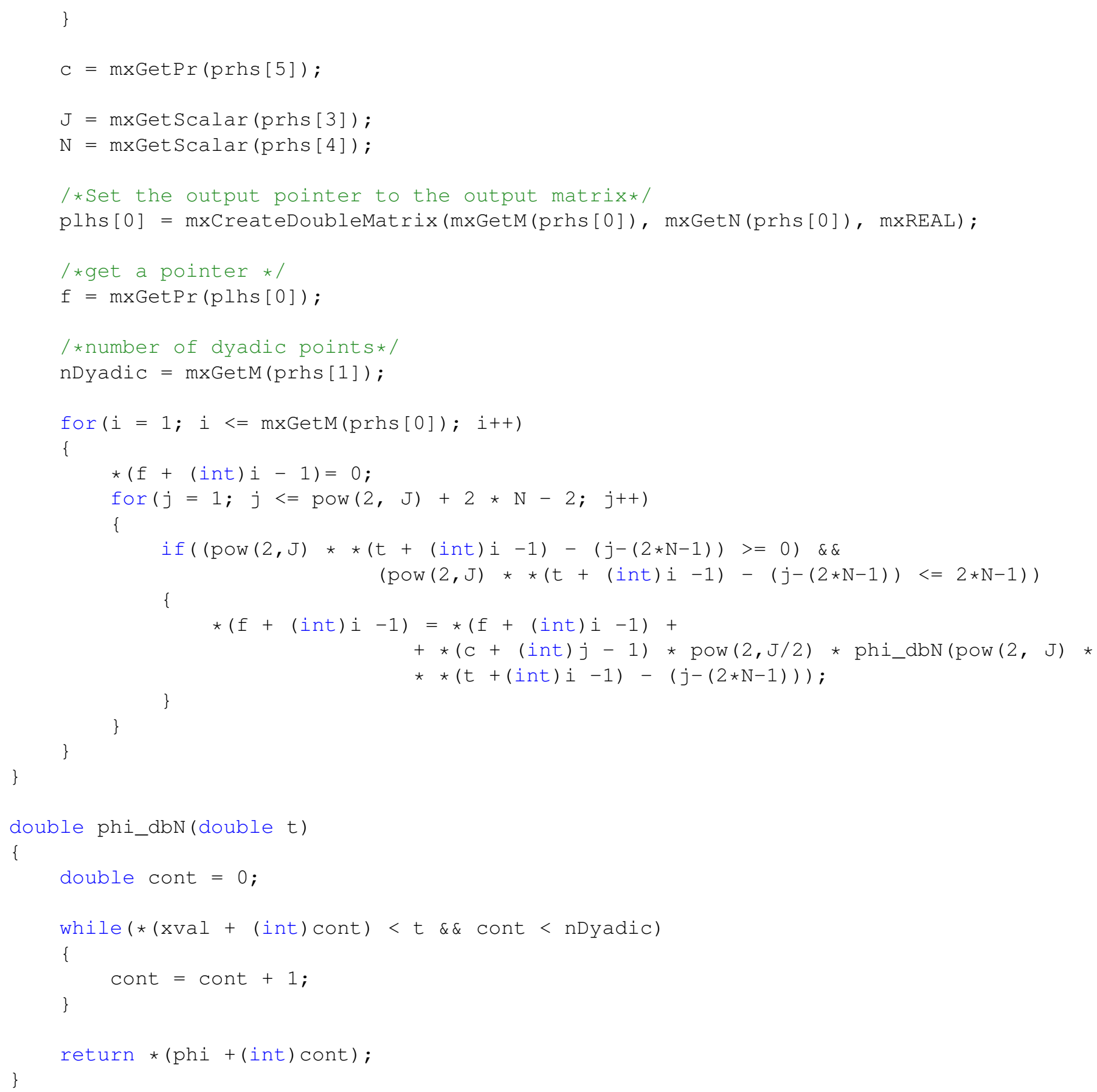

A.6 FieldHatchi_dbN

\section{Objetivo}

Esta função computa o valor da curva $\chi_{\boldsymbol{s}_{0}}(t)$ em uma localização não monitorada no vetor coluna de pontos $t$ dado a diagonal das matrizes $\Lambda_{i}$ e os coeficientes das curvas da amostra funcional $\chi_{\boldsymbol{s}_{i}}(t), i=1, \ldots, n$.

\section{Sintaxe}




\section{Descrição}

A função FieldHatchi_dbN tem cinco argumentos

i. té um vetor coluna $\left(t_{1}, \ldots, t_{m}\right)^{\top} \operatorname{com} t_{j} \in[0,1], j=1, \ldots, m$

ii. mLamb da é uma matriz em que a i-ésima coluna é a diagonal da matriz $\Lambda_{i}$ descrita no modelo de krigagem da Seção 3.3

iii. mcoef é a matriz de coeficientes estimados das curvas $\chi_{\boldsymbol{s}_{i}}(t), \ldots, \chi_{\boldsymbol{s}_{n}}(t)$ da amostra funcional

iv. Iter é um número inteiro positivo em que $\frac{1}{2^{\text {Iter }}}$ é a precisão usada na aproximação numérica de integrais envolvendo a função escala $\phi(x)$

e retorna um vetor coluna output_s $0=\left(\chi_{\boldsymbol{s}_{0}}\left(t_{1}\right), \ldots, \chi_{\boldsymbol{s}_{0}}\left(t_{m}\right)\right)^{\top}$ de mesma dimensão do vetor t.

\section{Código Fonte}

Listing A.6: Arquivo FieldHatchi_dbN.

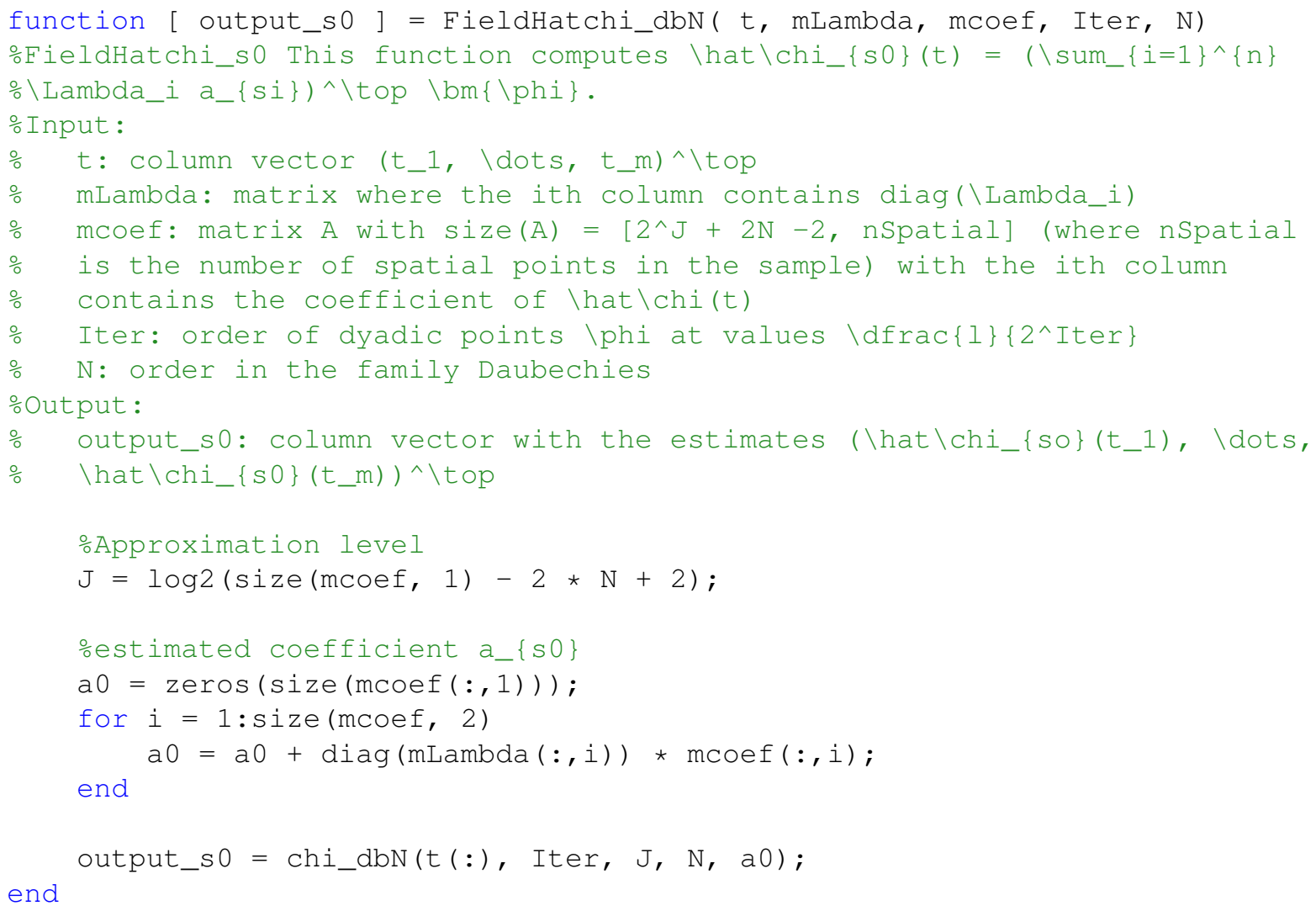

\section{A.7 FunctionalKriging}

\section{Objetivo}

Esta função é a implementação do método de Krigagem Ordinária Funcional (vide Seção 3.1 para maiores detalhes) usando ondaletas Haar. 


\section{Sintaxe}

[ lambda, mcoef, pontos, fEst] = Functionalkriging( lat, long, latData,...

longData, mdata, J, lplot, m) ;

\section{Descrição}

A função FunctionalKriging tem oito argumentos de entrada

i. lat é a latitude do ponto não monitorado $s_{0}$

ii. long é a longitude ponto não monitorado $s_{0}$

iii. latData é um vetor coluna contendo a latitude dos pontos $\boldsymbol{s}_{1}, \ldots, \boldsymbol{s}_{n}$ de nossa amostra funcional

iv. longData é um vetor coluna contendo a longitude dos pontos $\boldsymbol{s}_{1}, \ldots, \boldsymbol{s}_{n}$ de nossa amostra funcional

v. mdata é uma matriz em que a i-ésima coluna contém a série temporal observada no ponto $\boldsymbol{s}_{i}$ $\left(\chi_{\boldsymbol{s}_{i}}\left(t_{1}\right), \ldots, \chi_{\boldsymbol{s}_{i}}\left(t_{m}\right)\right)^{\top}$. Se $m$ não é potência de 2 , completamos com zeros a série até que $m^{\star}$ seja potência de 2 , isto é, a série temporal usada pelo programa é dada por $\left(\chi_{\boldsymbol{s}_{i}}\left(t_{1}\right), \ldots, \chi_{\boldsymbol{s}_{i}}\left(t_{m}\right), \chi_{\boldsymbol{s}_{i}}\left(t_{m+1}\right), \ldots\right.$ $\operatorname{com} m^{\star}=\left\lceil\log _{2}(n)\right\rceil$ e $\chi_{\boldsymbol{s}_{i}}\left(t_{j}\right)=0$ para $j=m+1, \ldots, m^{\star}$

vi. J é o nível de aproximação.

vii. Ipl ot é uma variável lógica. Se true, imprime dois gráficos: um contendo a curva estimada e um mostrando os valores estimados dos parâmetros $\lambda_{1}, \ldots, \lambda_{n}$. Esta variável é opcional e seu valor padrão é true.

viii. m é uma variável inteira positiva. Ela estabelece quantos pontos serão usado para fazer o gráfico da curva estimada, caso a variável lplot seja verdadeira. Esta variável é opcional e seu valor padrão é 1000.

e retorna quatro matrizes

a. lambda é um vetor coluna $\left(\lambda_{1}, \ldots, \lambda_{n}\right)^{\top}$ contendo os parâmetros estimados do modelo de Krigagem Ordinária Funcional.

b. mcoef é uma matriz contendo os coeficientes da curvas da amostra funcional usando ondaletas. A i-ésima linha contém os coeficientes da projeção da curva $\chi_{\boldsymbol{s}_{i}}(t)$ no ponto $\boldsymbol{s}_{i}$ no espaço de aproximação $V_{J}$.

c. pontos é um vetor coluna $\left(t_{1}, \ldots, t_{m}\right)^{\top}$ contendo as abscissas usadas para produzir o gráfico da curva estimada caso lplot seja true.

d. é um vetor coluna $\left(\chi_{\boldsymbol{s}_{0}}\left(t_{1}\right), \ldots, \chi_{\boldsymbol{s}_{0}}\left(t_{m}\right)\right)^{\top}$ contendo as ordenadas usadas para gerar o gráfico da curva suavizada caso lplot tenha valor true. 


\section{Código Fonte}

Listing A.7: Arquivo FunctionalKriging.m.

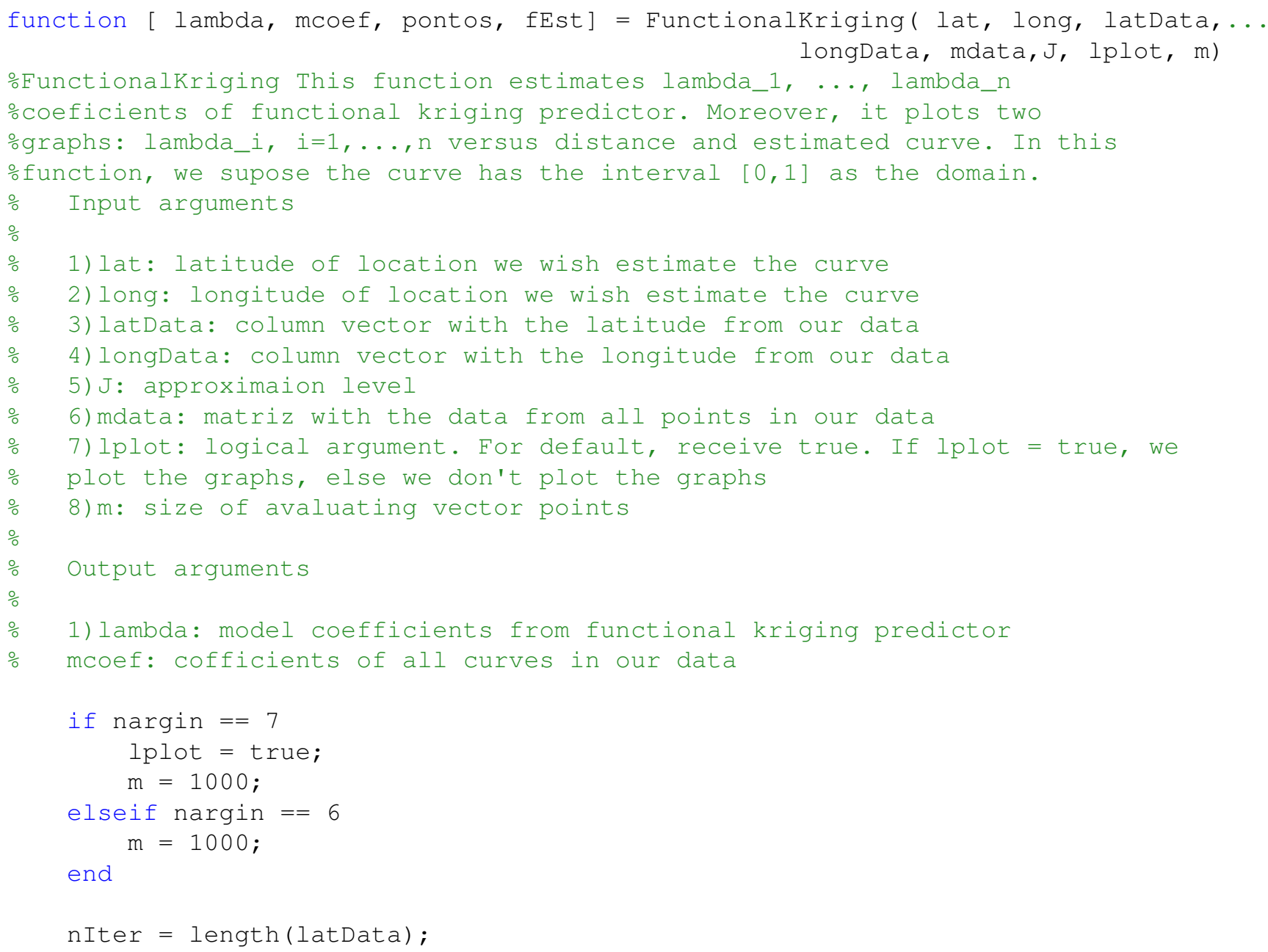




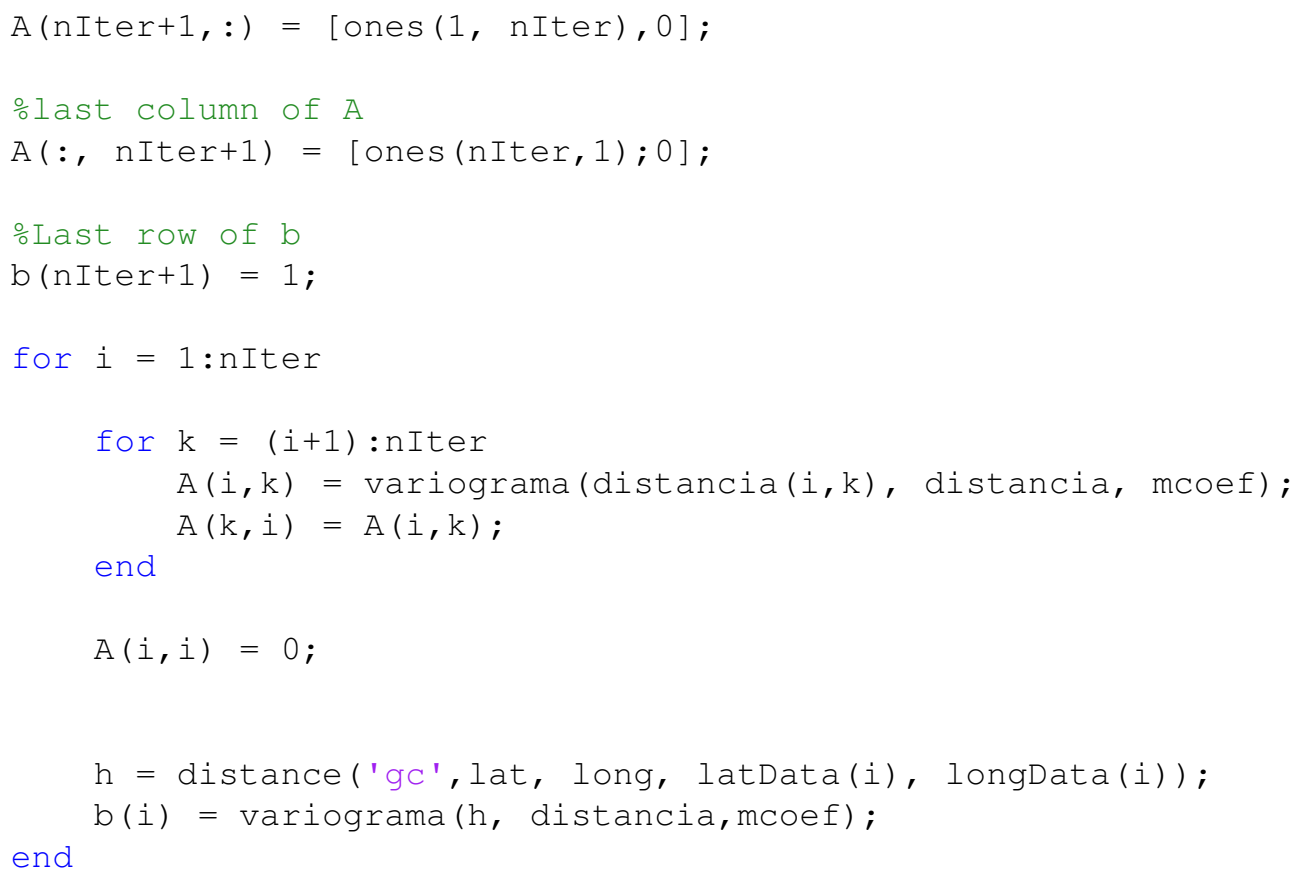


\%The second argument is optional and has default value 0.1

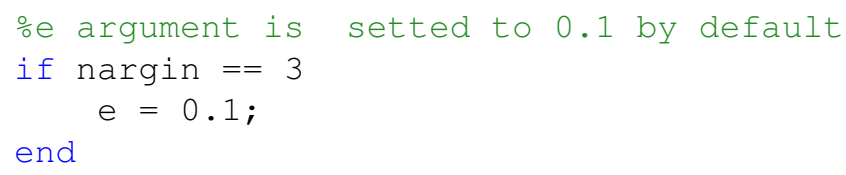


valor $=$ valor $* 2^{\wedge}(\mathrm{J} / 2) / 2^{\wedge} \mathrm{n}$;

$\mathrm{c}(\mathrm{k}+1)=$ valor;

end

$$
\text { end }
$$

\section{A.8 hatchi_so_haar}

\section{Objetivo}

Esta função tem o objetivo de calcular a estimativa da curva $\chi_{s_{0}}(t)$ em um ponto $t \in[0,1]$ usando os coeficientes $\lambda_{1}, \ldots, \lambda_{n}$ obtidos pelo médoto de interpolação espacial denominado Krigagem Ordinária Funcional e os coeficientes de aproximação das curvas $\chi_{\boldsymbol{s}_{i}}(t), i=1, \ldots, n$ de nossa amostra funcional.

\section{Sintaxe}

fEst $=$ hatchi_s0_haar (pontos, mcoef, lambda, J)

\section{Descrição}

Esta função tem quatro argumentos

i. pontos é um vetor coluna $\left(t_{1}, \ldots, t_{m}\right)^{\top} \operatorname{com} t_{j} \in[0,1], j=1, \ldots, m$ em que desejamos calcular $\chi_{\boldsymbol{s}_{0}}\left(t_{j}\right), j=1, \ldots, m$

ii. mcoef é uma matriz em que a i-ésima coluna contém os coeficientes de aproximação da curva $\chi_{\boldsymbol{s}_{i}}(t)$ de nossa amostra funcional

iii. lambda é um vetor coluna $\left(\lambda_{1}, \ldots, \lambda_{n}\right)^{\top}$ contendo os parâmetros estimados do modelo de Krigagem Ordinária Funcional

iv. J é o nível de aproximação

e retorna como saída um vetor coluna $\left(\chi_{\boldsymbol{s}_{0}}\left(t_{1}\right), \ldots, \chi_{\boldsymbol{s}_{0}}\left(t_{m}\right)\right)^{\top}$ de mesma dimensão do vetor $\mathrm{t}$.

\section{Código Fonte}

Listing A.8: Arquivo hatchi_s0_haar.m

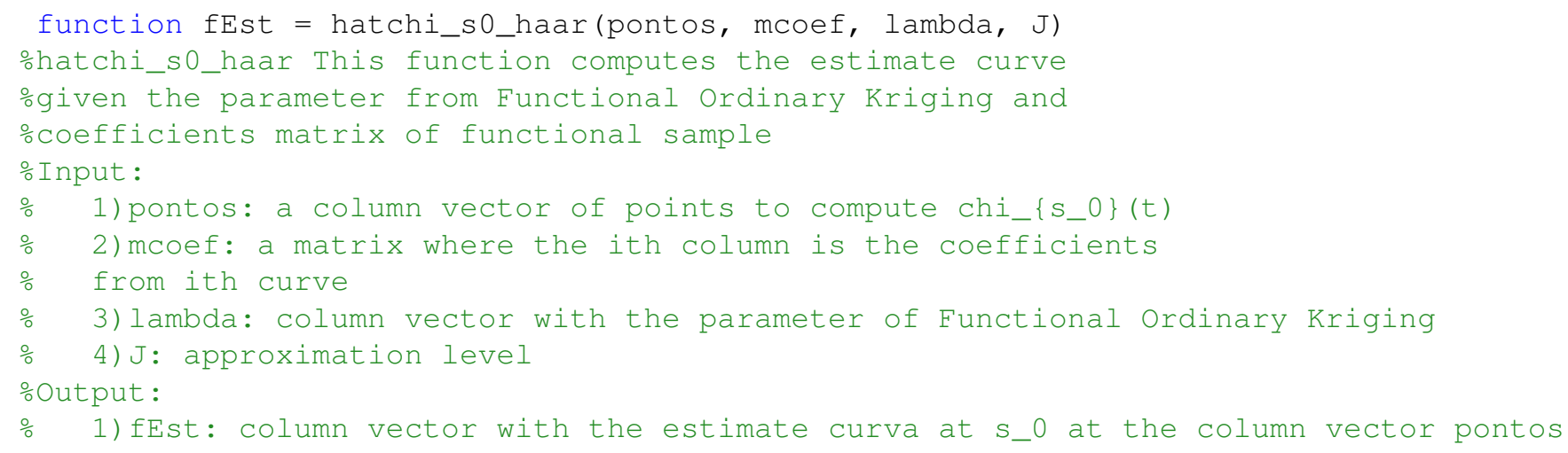




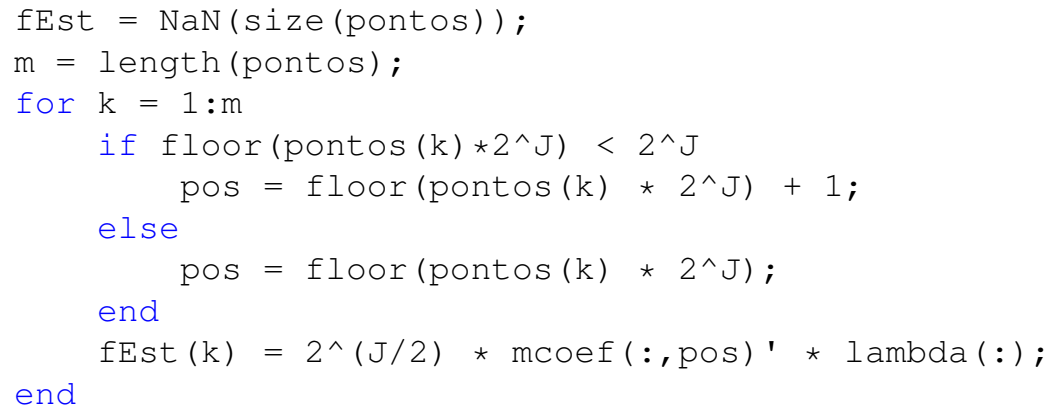

end

\section{A.9 PointwiseFuncKriging}

\section{Objetivo}

Esta função é uma implementação do método de Krigagem Tempo-Variante Funcional (vide Seção 3.2 para maiores detalhes deste modelo) para dados funcionais usando ondaletas Haar.

\section{Sintaxe}

[ mb, mcoef, pontos, f] = PointwiseFunckriging( lat, long, latData, longData,... mdata, J, lplot, m);

\section{Descrição}

A função PointwiseFunctKringing tem oito argumentos de entrada

i. lat é a latitude do ponto não monitorado $s_{0}$

ii. long é a longitude ponto não monitorado $s_{0}$

iii. latData é um vetor coluna contendo a latitude dos pontos $\boldsymbol{s}_{1}, \ldots, \boldsymbol{s}_{n}$ de nossa amostra funcional

iv. longData é um vetor coluna contendo a longitude dos pontos $\boldsymbol{s}_{1}, \ldots, \boldsymbol{s}_{n}$ de nossa amostra funcional

v. mdata é uma matriz em que a i-ésima coluna contém a série temporal observada no ponto $\boldsymbol{s}_{i}$ $\left(\chi_{\boldsymbol{s}_{i}}\left(t_{1}\right), \ldots, \chi_{\boldsymbol{s}_{i}}\left(t_{m}\right)\right)^{\top}$. Se $m$ não é potência de 2, completamos com zeros a série até que $m^{\star}$ seja potência de 2 , isto é, a série temporal usada pelo programa é dada por $\left(\chi_{\boldsymbol{s}_{i}}\left(t_{1}\right), \ldots, \chi_{\boldsymbol{s}_{i}}\left(t_{m}\right), \chi_{\boldsymbol{s}_{i}}\left(t_{m+1}\right)\right.$ com $m^{\star}=\left\lceil\log _{2}(n)\right\rceil$ e $\chi_{\boldsymbol{s}_{i}}\left(t_{j}\right)=0$ para $j=m+1, \ldots, m^{\star}$.

vi. J é o nível de aproximação.

vii. lpl ot é uma variável lógica. Se true, imprime dois gráficos: um contendo a curva estimada e um mostrando as funções estimadas $\lambda_{1}(t), \ldots, \lambda_{n}(t)$. Esta variável é opcional e seu valor padrão é true.

viii. m é uma variável inteira positiva. Ela estabelece quantos pontos serão usado para fazer o gráfico da curva estimada, caso a variável lplot seja verdadeira. Esta variável é opcional e seu valor padrão é 1000 . 
e retorno quatro saídas

a. mb é uma matriz mb em que a i-ésima coluna contém o parâmetro estimado $\boldsymbol{b}_{i}$

b. mcoef é uma matriz mcoef em que a i-ésima coluna contém os coeficientes da projeção da curva $\chi_{\boldsymbol{s}_{i}}(t)$ no espaço de aproximação $V_{J}$

c. pontos é um vetor coluna $\left(t_{1}, \ldots, t_{m}\right)^{\top}$ que é composto por $m$ pontos igualmente espaçados entre 0 e 1

d. f é um vetor coluna $\left(\chi_{\boldsymbol{s}_{0}}\left(t_{1}\right), \ldots, \chi_{\boldsymbol{s}_{0}}\left(t_{m}\right)\right)^{\top}$ compreendendo o valores estimados da curva $\chi_{s_{0}}(t)$ no ponto não monitorado $s_{0}$ para cada entrada do vetor coluna pontos

Listing A.9: Arquivo PointwiseFunckriging.m.

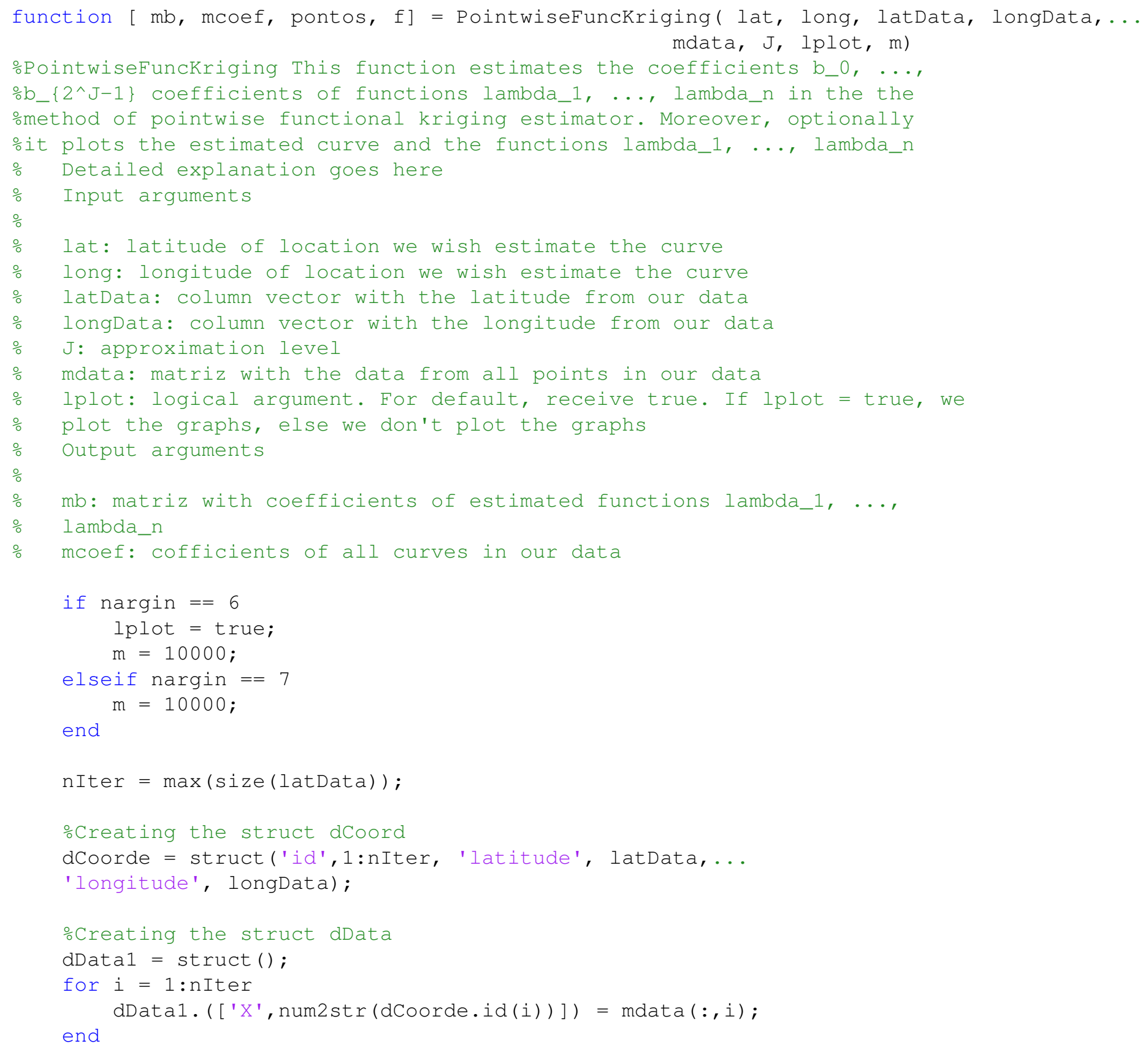




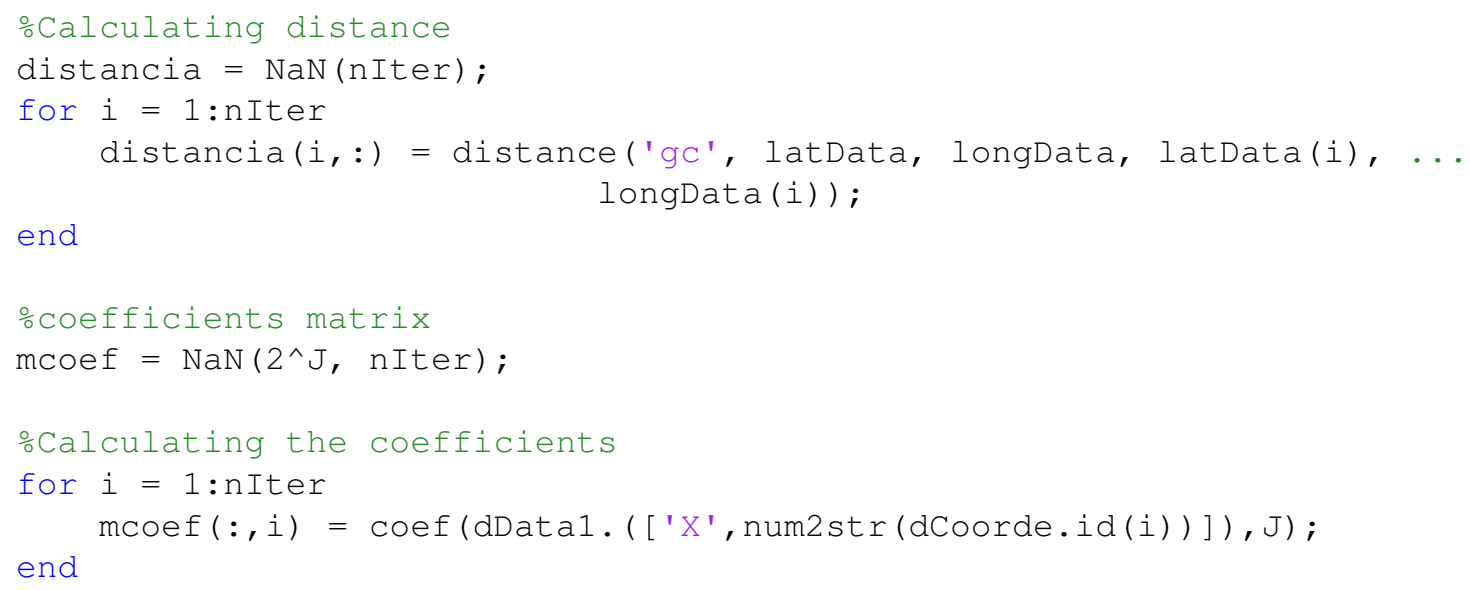




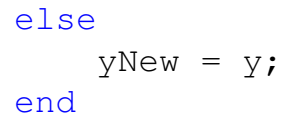




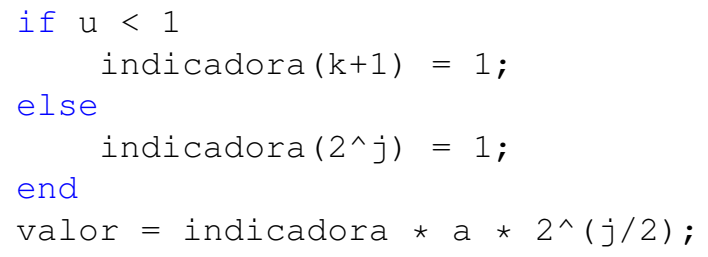

\section{A.10 FTVK_hatchi_s0_haar}

\section{Objetivo}

Objetivo dessa função é estimar o valor da curva $\chi_{s_{0}}(t)$ em um ponto não monitorado $\boldsymbol{s}_{0}$ para $t \in[0,1]$ usando o método Krigagem Tempo-Variante Funcional. Mais precisamente, estimamos $\chi_{s_{0}}(t)$ em $t \in[0,1]$ por

$$
\chi_{\boldsymbol{s}_{0}}(t)=\sum_{i=1}^{n} \hat{\lambda}_{i}(t) \hat{\chi}_{\boldsymbol{s}_{i}}(t)
$$

em que $\hat{\lambda}_{i}(t)=\sum_{k} b_{i, k}^{J} \phi_{J, k}(t)$ e $\hat{\chi}_{\boldsymbol{s}_{i}}(t)=\sum_{k} a_{i, k}^{J} \phi_{J, k}(t)$ (para maiores detalhes vide Seção 3.2).

\section{Sintaxe}

fEst $=$ FTVK_hatchi_s0_haar(pontos, mcoef, mb, J)

\section{Descrição}

Esta função tem quatro argumentos

i. pontos é um vetor coluna $\left(t_{1}, \ldots, t_{m}\right)^{\top} \operatorname{com} t_{j} \in[0,1], j=1, \ldots, m$ em que desejamos calcular $\chi_{s_{0}}\left(t_{j}\right), j=1, \ldots, m$

ii. mcoef é uma matriz em que a i-ésima coluna contém os coeficientes de aproximação da curva $\chi_{\boldsymbol{s}_{i}}(t)$ de nossa amostra funcional

iii. mb é uma matriz em que a i-ésima coluna contém os coeficientes estimados de $\lambda_{i}(t)$, isto é, a i-ésima é constituda por $b_{i,-M}, \ldots, b_{i, M}$

iv. J é o nível de aproximação

e retorna como saída um vetor coluna $\left(\chi_{\boldsymbol{s}_{0}}\left(t_{1}\right), \ldots, \chi_{\boldsymbol{s}_{0}}\left(t_{m}\right)\right)^{\top}$ de mesma dimensão do vetor $t$.

\section{Código Fonte}

Listing A.10: Arquivo FTVK_hatchi_s0_haar.m 


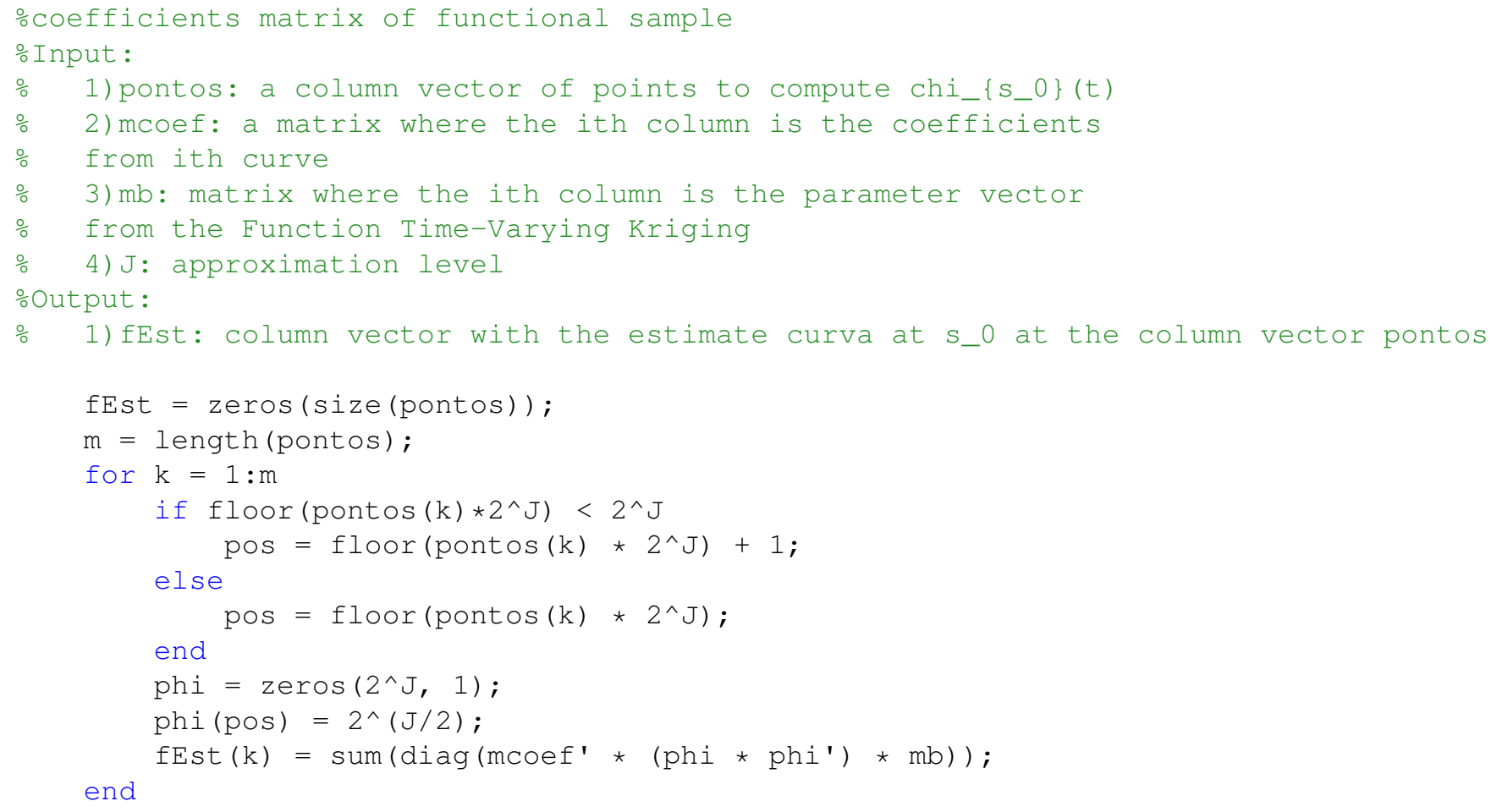

end

\section{A.11 coef_Haar}

\section{Objetivo}

Dado o vetor coluna $\mathrm{y}=\left(y_{1}, \ldots, y_{n}\right)^{\top}$, esta função retorna os coeficientes da aproximação $\hat{f}$ usando regressão não paramétrica e ondaletas Haar.

\section{Sintaxe}

$\mathrm{C}=\operatorname{coefHaar}(\mathrm{y}, \mathrm{J})$;

\section{Descrição}

A função coefHaar tem dois argumentos de entrada

i. y é um vetor coluna contendo a série temporal $y_{1}, \ldots, y_{n}$ em que desejamos suavizar usando regressão não paramétrica

ii. J é o nível de aproximação na análise de multirresolução

e retorna um vetor coluna $\mathrm{c}$ com $2^{\mathrm{J}}$ linhas que são os coeficientes estimados da função $\hat{f}$ obtidos usando regressão não paramétrica conforme Ogden (1997).

\section{Código Fonte}


Listing A.11: Arquivo coefHaar.m.

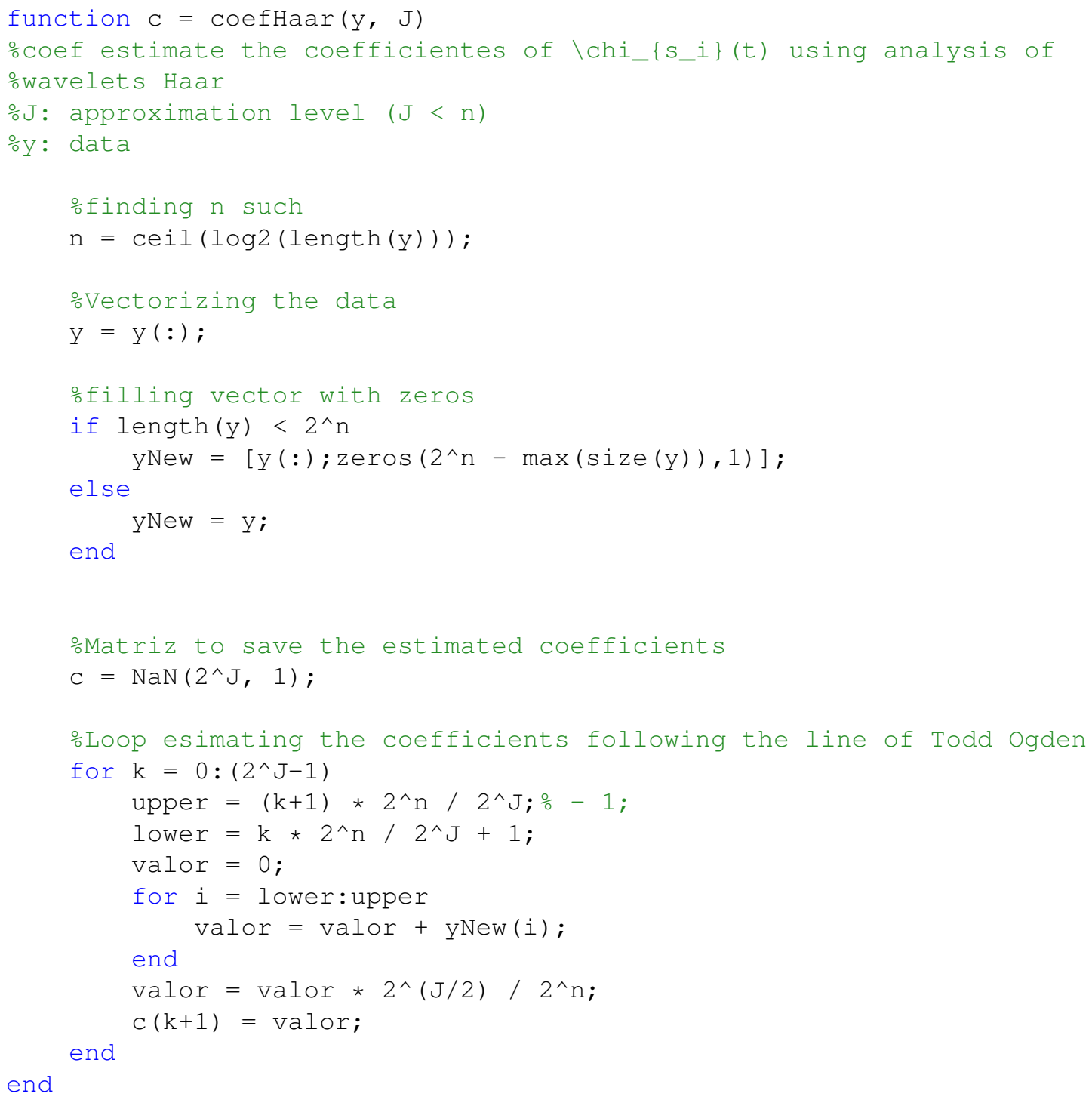

\section{A.12 coef_dbN}

\section{Objetivo}

Esta função calcula os coeficientes da função $\hat{f}(x)=\sum_{k} c_{k} \phi_{J, k}(t)$ obtida pela regressão paramétrica usando ondaletas Daubechies dada por

$$
y_{i}=f\left(\frac{i}{n}\right)+\epsilon_{i}, \quad i=1, \ldots, n .
$$

\section{Sintaxe}

$\mathrm{c}=$ coef_dbN( $\mathrm{y}$, Iter, J, $\mathrm{N})$

\section{Descrição}

A função coef_dbN tem quatro argumentos de entrada 
i. y é um vetor coluna composta da série temporal $y_{1}, \ldots, y_{n}$ que desejamos suavizar

ii. Iter é um número inteiro positivo inteiro em que $\frac{1}{2^{\text {Iter }}}$ é a precisão usada nas integrações numéricas

iii. J é o nível de aproximação

iv. N é um número inteiro positivo indexando a família Daubechies

e retorna um vetor coluna $\mathrm{c}=\left(c_{-2 N+2}, \ldots, c_{2^{J}-1}\right)^{\top} \operatorname{com} 2^{\mathrm{J}}+2 N-2$ elementos tal que $\hat{f}(x)=$ $\sum_{k=-2 N+2}^{2^{J}-1} c_{k} 2^{\frac{J}{2}} \phi\left(2^{J} x-k\right)$ em que $\phi(\cdot)$ é função escala para ondaleta Daubechies de ordem $N$.

\section{Código Fonte}

Listing A.12: Arquivo coef_dbN

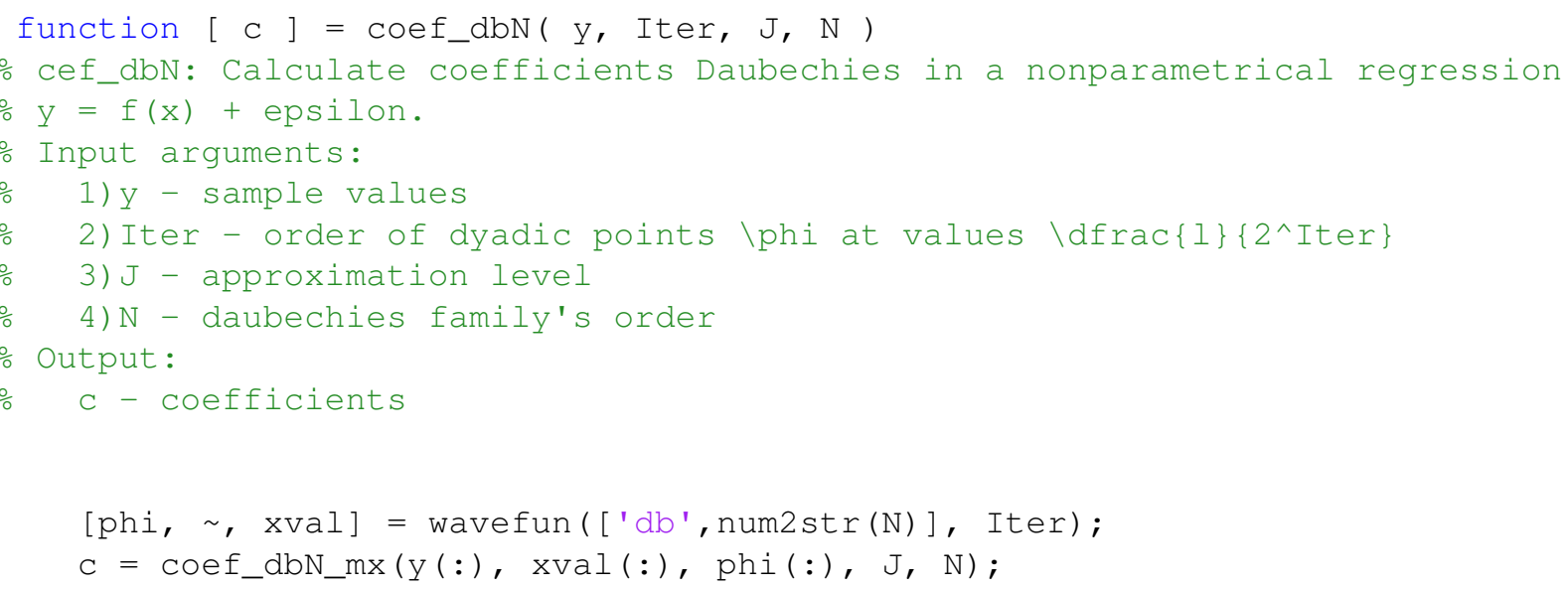

Note que a função coef_dbN cujo código está descrito em Listing A.12 usa o MEX file descrito em Listing A.13.

Listing A.13: Arquivo coef_dbN_mx.c.

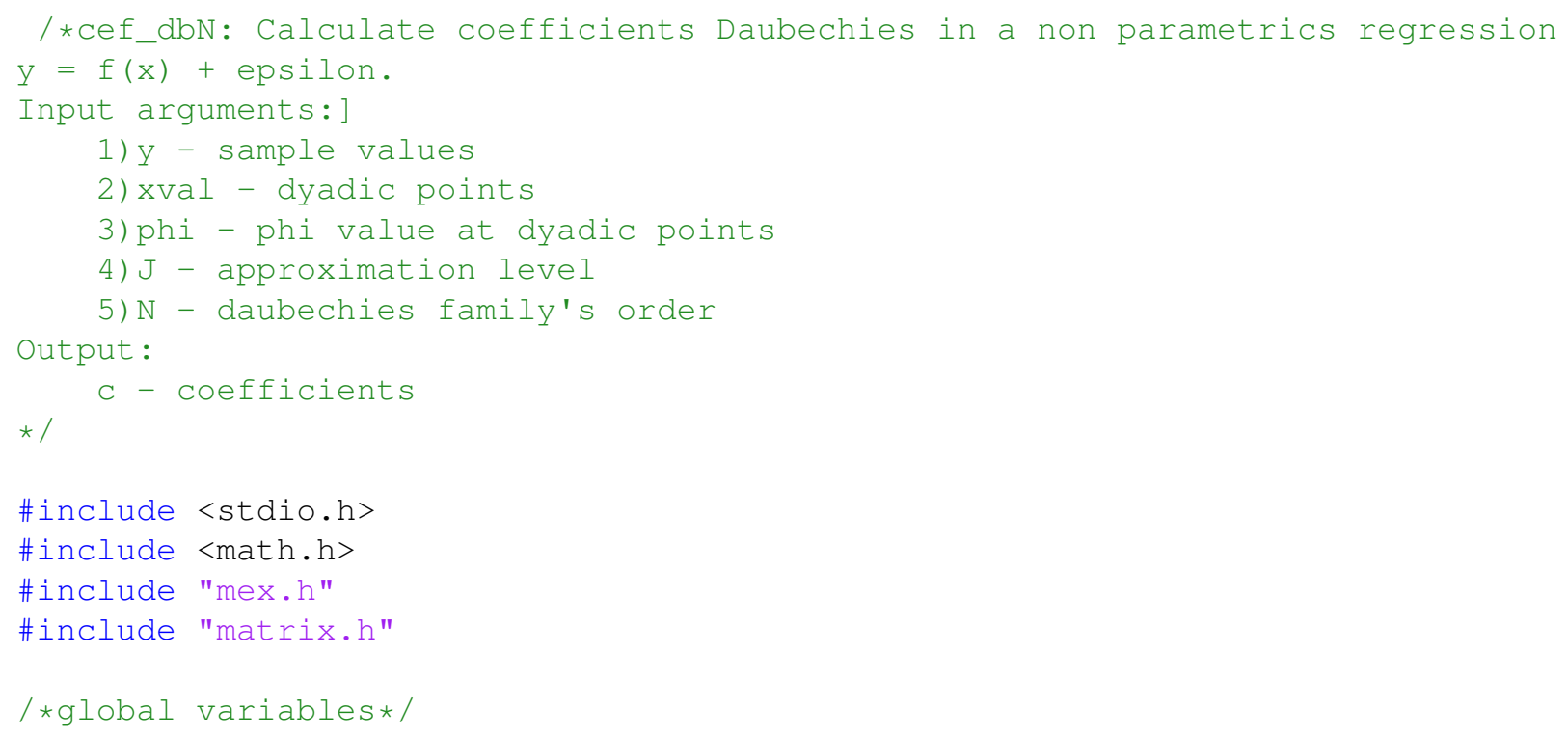




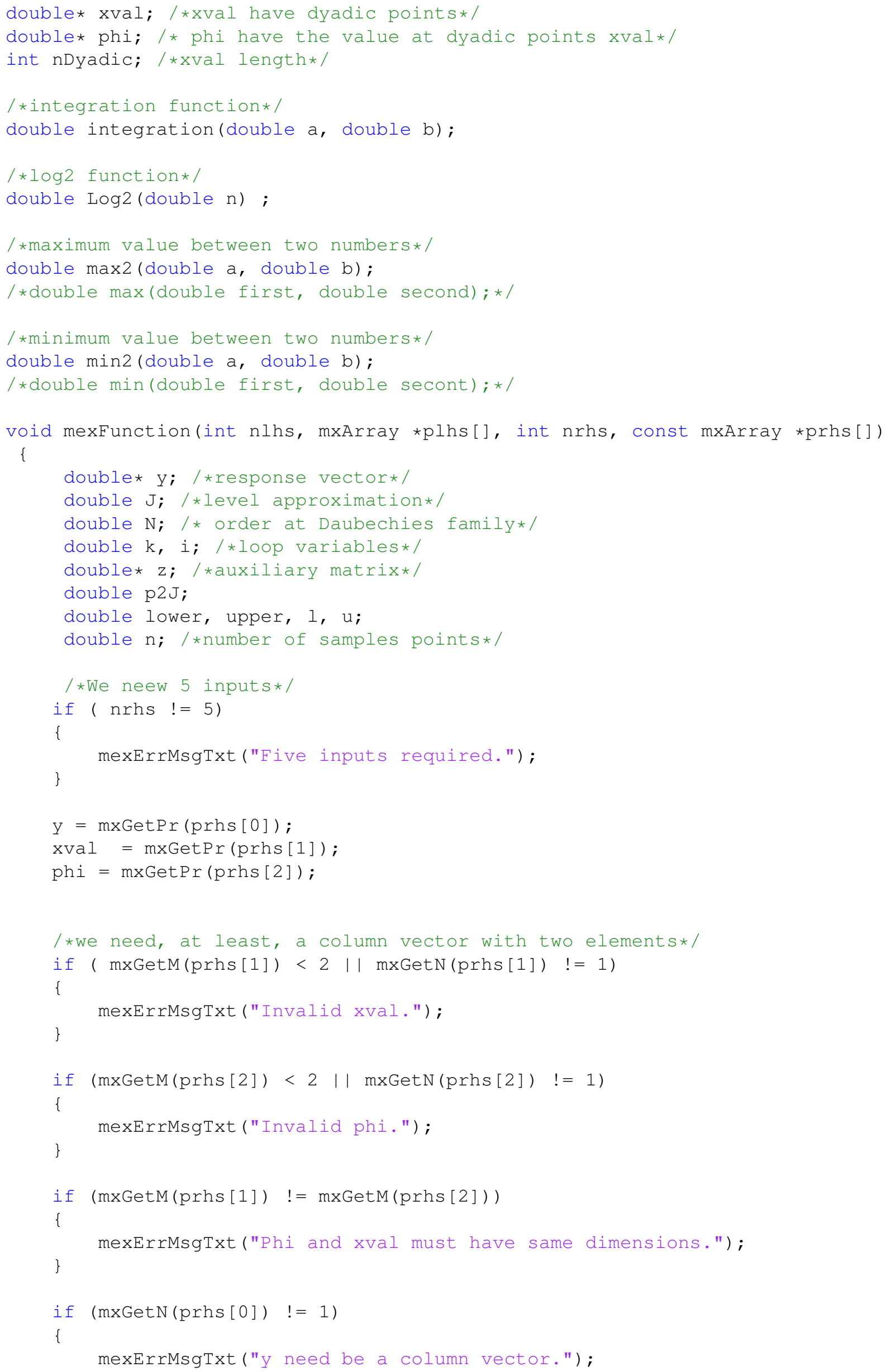




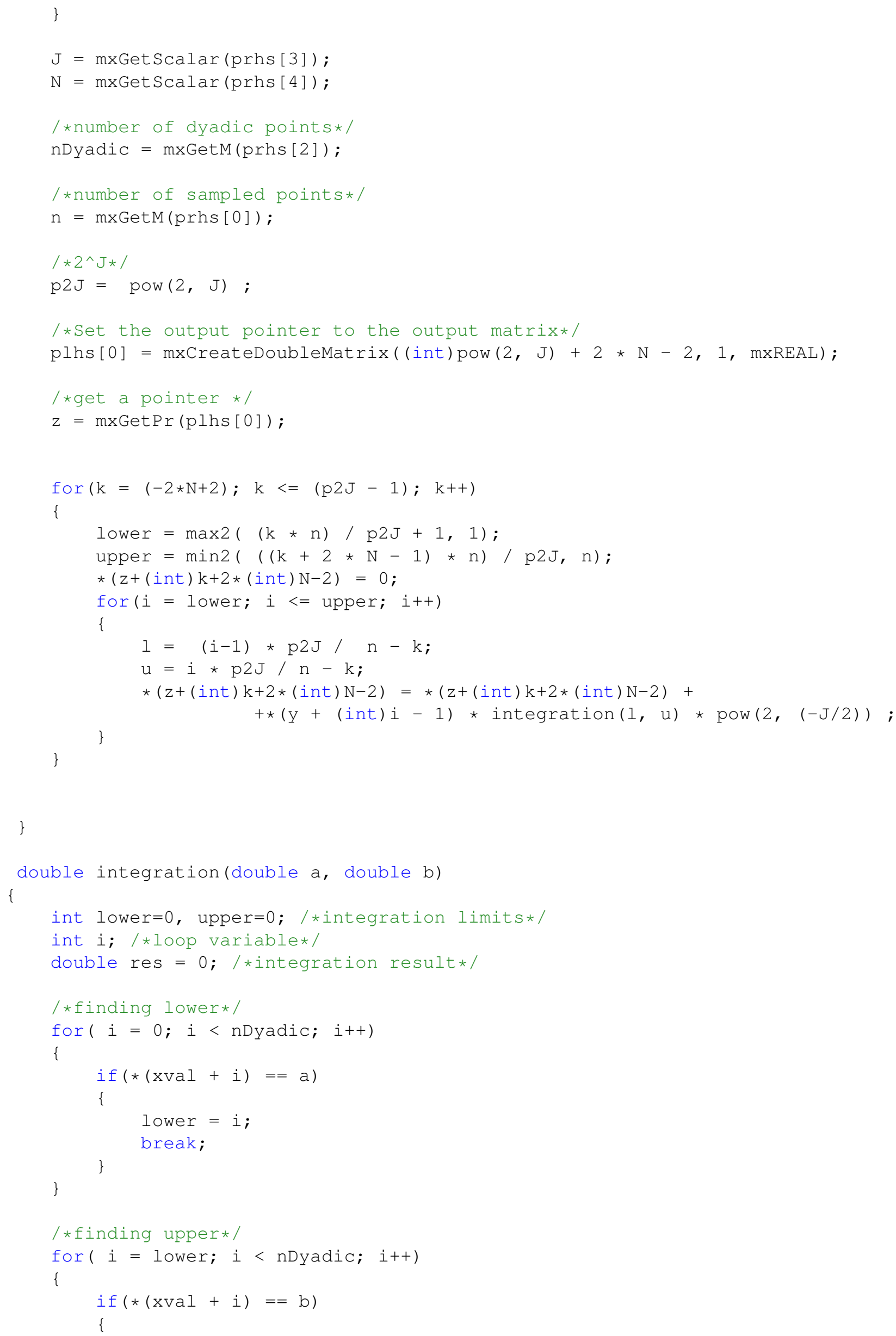




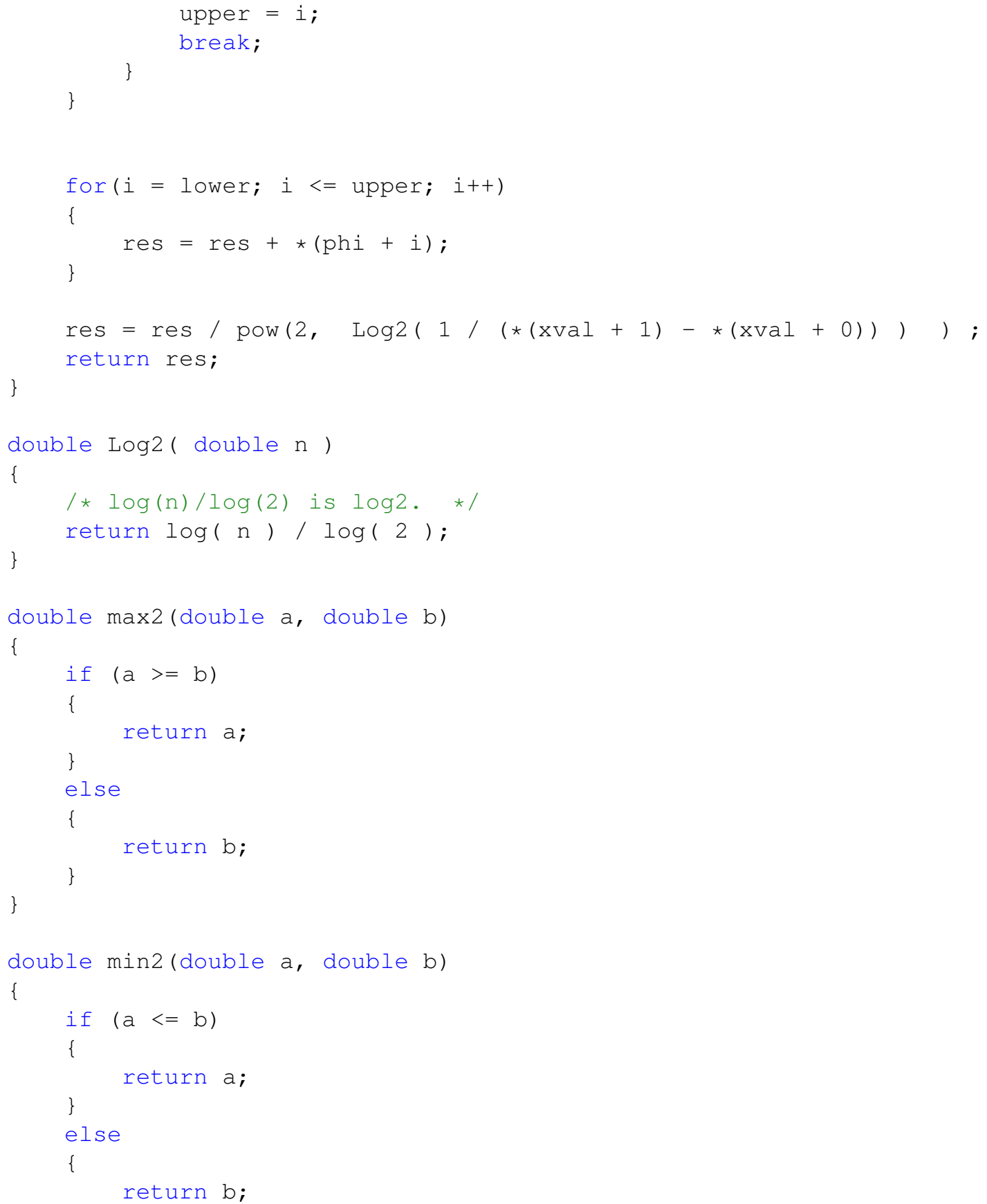

\section{A.13 FuncKriging_dbN}

\section{Objetivo}

Esta função implmenenta o método de Krigagem Tempo-Variante Funcional usando ondaletas da família Daubechies.

\section{Sintaxe}

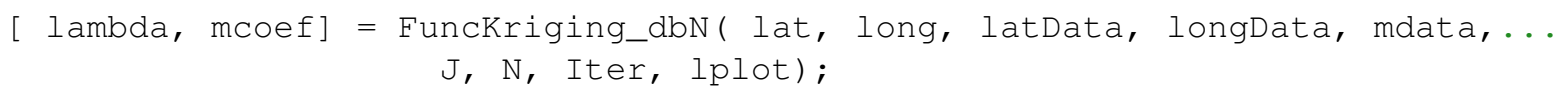




\section{Descrição}

Esta função tem nove argumentos de entrada

i. lat é a latitude do ponto não monitorado $s_{0}$

ii. long é a longitude do ponto não monitorado $s_{0}$

iii. latData é um vetor coluna contendo as latitudes dos pontos $\boldsymbol{s}_{1}, \ldots, \boldsymbol{s}_{n}$ das curvas em nossa amostra funcional

iv. longData é um vetor coluna contendo as longitudes dos pontos $s_{1}, \ldots, \boldsymbol{s}_{n}$ das curvas em nossa amostra funcional

v. mdata é um matriz em que a i-ésima coluna é a série temporal $\chi_{\boldsymbol{s}_{i}}\left(t_{1}\right), \ldots, \chi_{\boldsymbol{s}_{i}}\left(t_{n}\right)$ observada da curva $\chi_{\boldsymbol{s}_{i}}(t)$

vi. J é o nível de aproximação na análise de multirresolução

vii. $\mathrm{N}$ é um número inteiro positivo que indexa a ordem na família Daubechies

viii. Iter é uma número inteiro positivo tal que $\frac{1}{2^{\text {Iter }}}$ precisão numérica de integrais envolvendo a função escala $\phi(x)$. Este argumento é opcional e seu valor padrão é 10.

ix. lplot uma variável lógica. Se true, a função produz um gráfico com a curva estimada. Esta argumento é opcional e seu valor padrão é true.

e retorna duas saídas

a. lamb da é um vetor coluna composto dos parâmetros estimados $\lambda_{1}, \ldots, \lambda_{n}$ no modelo de Krigagem Ordinária Funcional

b. mcoef é uma matriz em que a i-ésima coluna contém os coeficientes estimados da curva $\chi_{\boldsymbol{s}_{i}}(t)$

\section{Código Fonte}

Listing A.14: Arquivo FuncKriging_dbn.m.

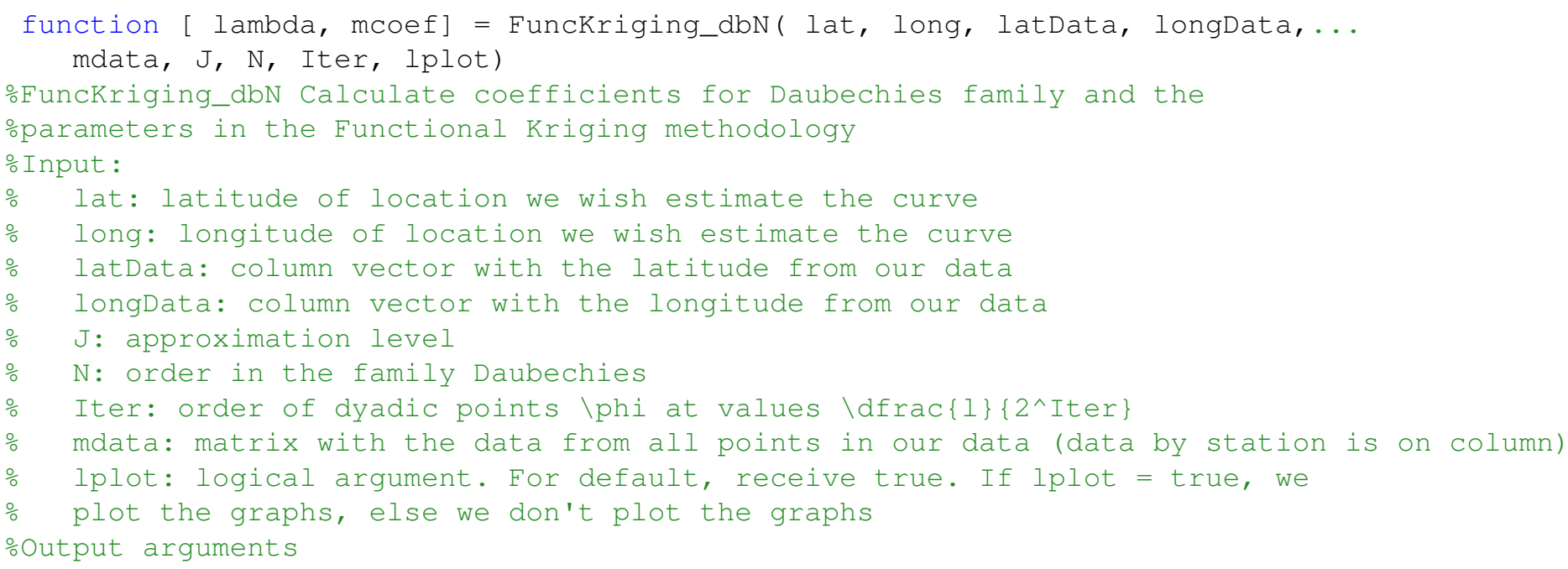




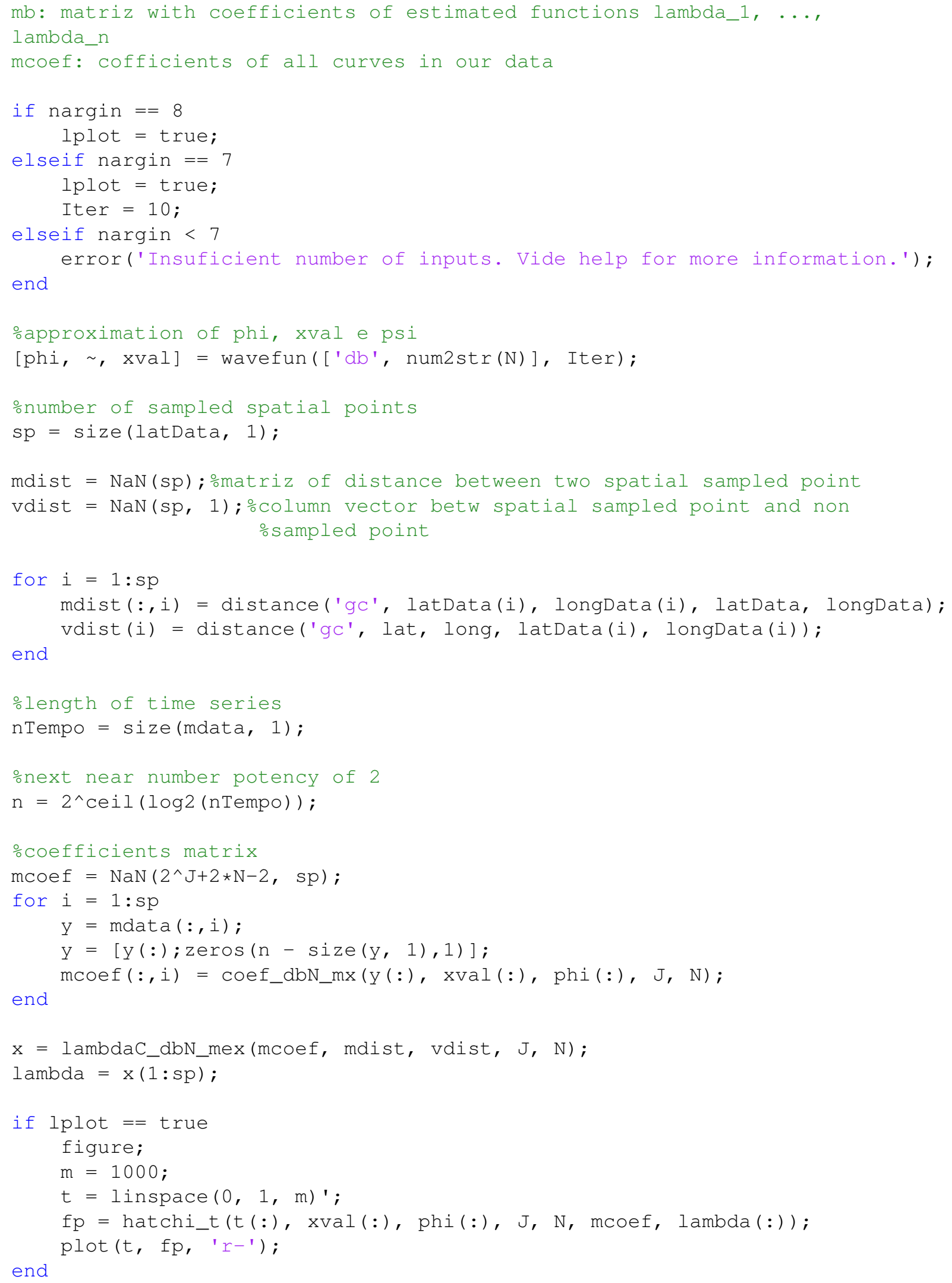

end

Note que a função FuncKriging_dbN, cujo código está descrito em Listing A.14, usa a função lambdaC_dbN_mex que calcula os parâmetros $\lambda_{1}, \ldots, \lambda_{n}$ no modelo de Krigagem Ordinária Funcional e foi implementa usando MEX file cujo código fonte está transcrito em Listing A.15. Ademais, para produzir o gráfico da curva ajustada usamos a função hatchi_t_mx 
que foi programada usando MEX file e seu código fonte está impresso no Listing A.17.

Listing A.15: Arquivo lambdaC_dbN_mex.c.

/*

lambdac: calculate \lambda_1, \dots, \lambda_n using the functional kriging predictor Inputs:

1) mcoef: matrix with rows representing coefficients of sampled spots

2) mdist: matrix $A=\left(a_{-}\{i j\}\right)$ with $a_{-}\{i j\}=\operatorname{distance}\left(s_{-} i, s_{-} j\right)$ where

s_i, s_j are sampled spots

3) vdist: column vector $V=\left(v_{-} 1, \quad \backslash \text { dots, } V \_n\right)^{\wedge} T$ with $V_{-j}=$

distance (s_0, $s_{-} j$ ) where $s_{-} j$ is a sampled spot

4) J: approximation level

5) N: order in the daubechies family output:

1) lambda: estimated coefficients of functional kriging predictor * 1

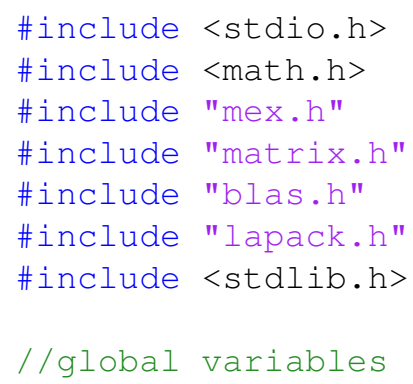




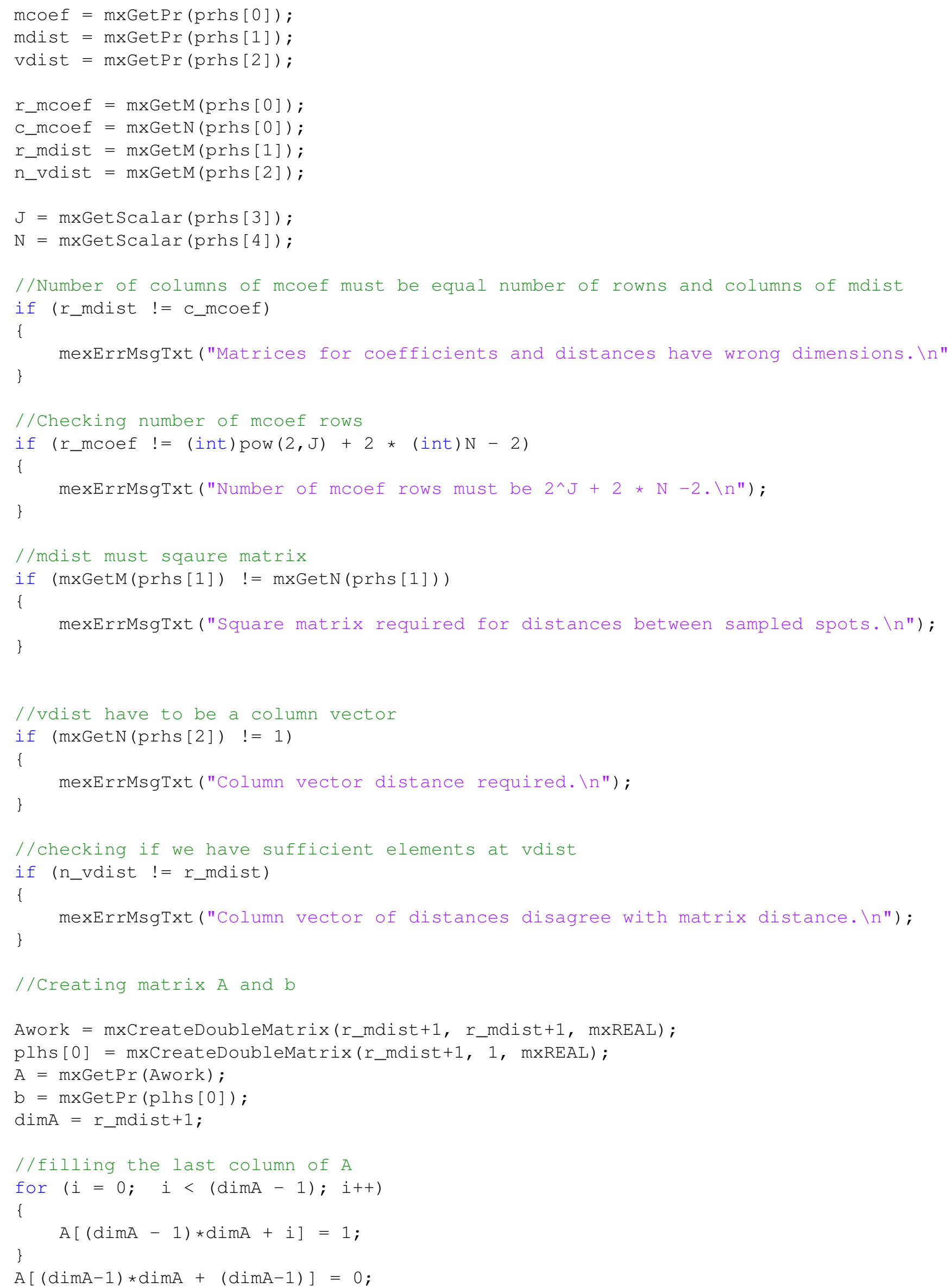




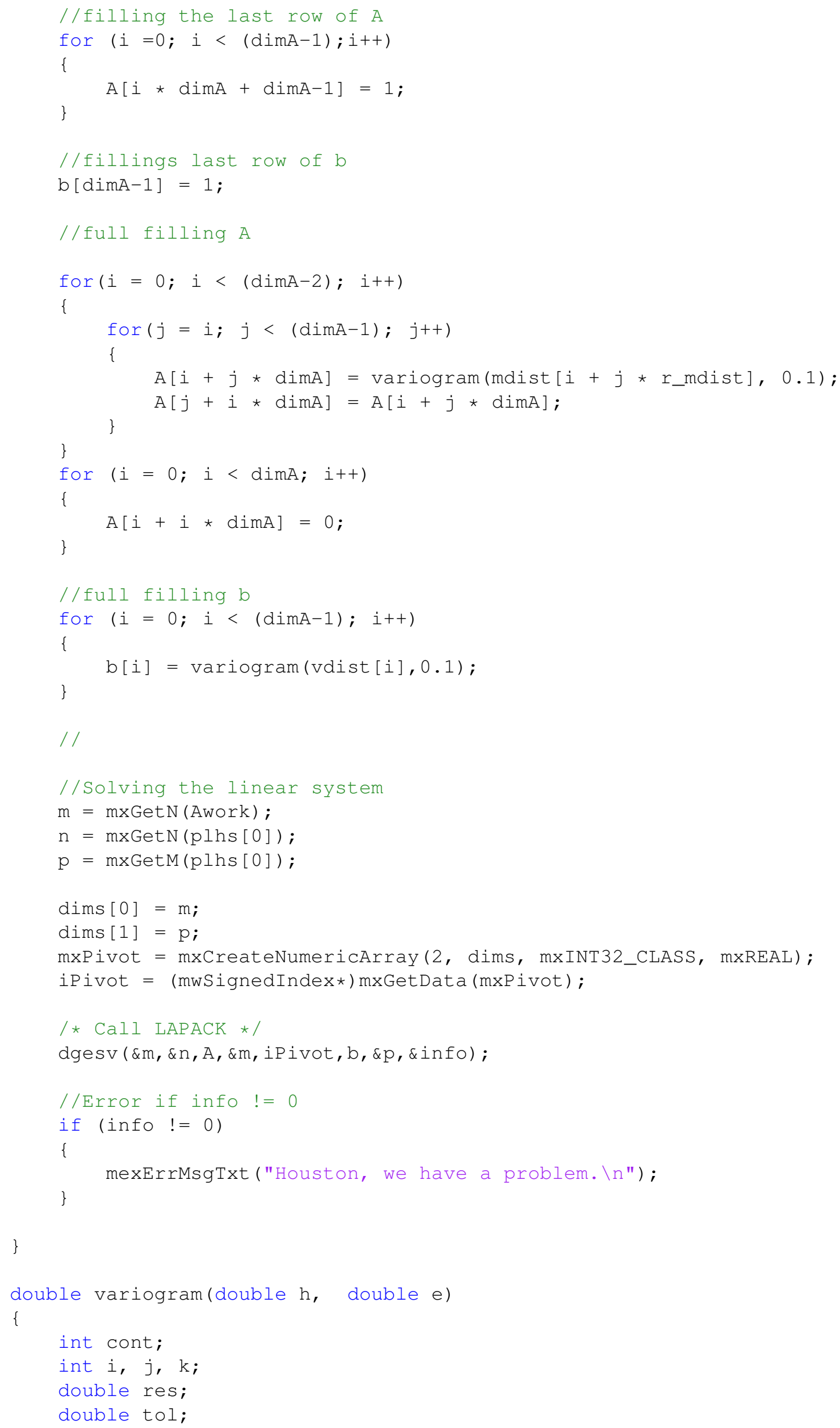




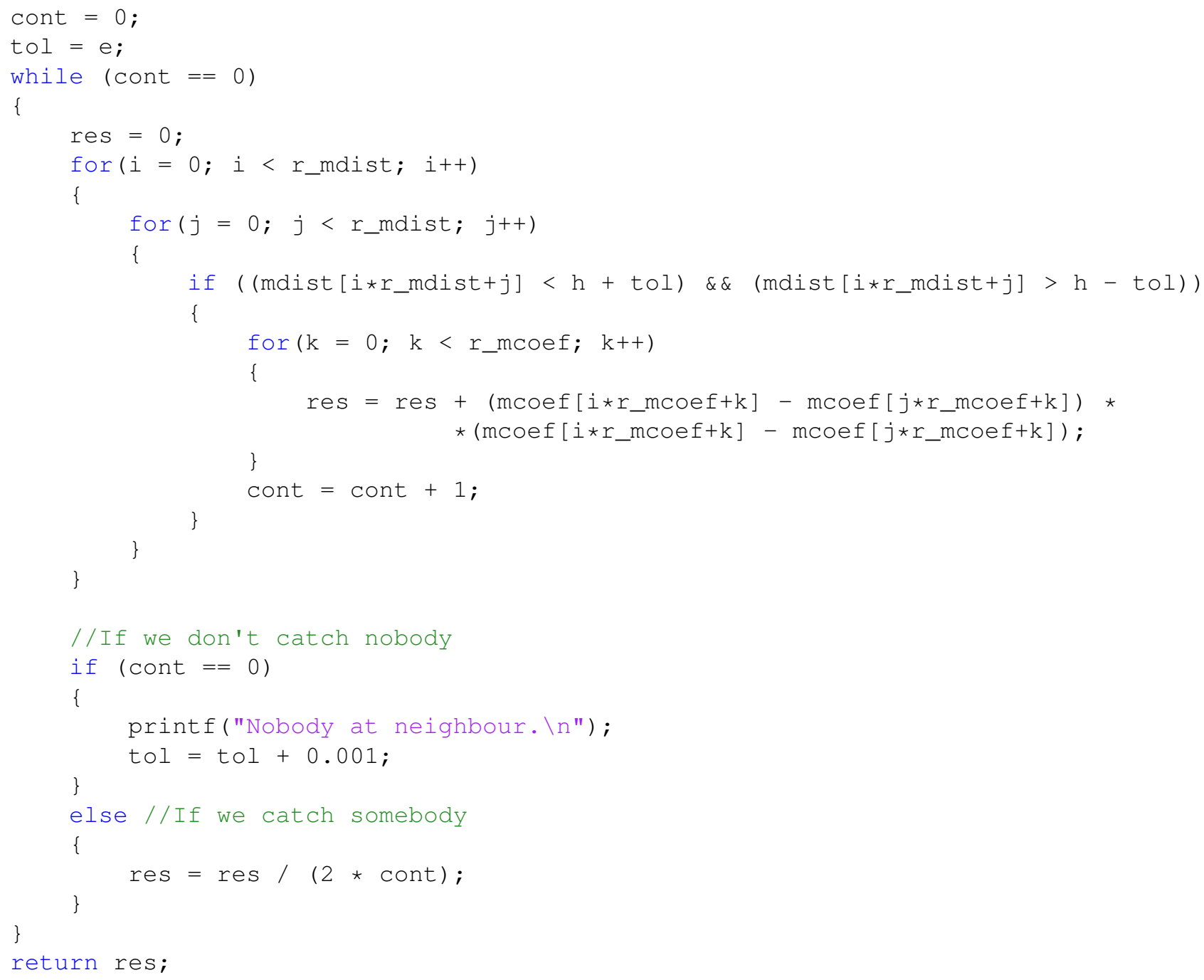

\section{A.14 hatchi_s0}

\section{Objetivo}

Esta função calcula o valor da curva $\chi_{\boldsymbol{s}_{0}}(t)$ no ponto $t$ dado os parâmetros $\lambda_{1}, \ldots, \lambda_{n}$ no modelo de Krigagem Ordinária Funcinal.

\section{Sintaxe}

$\mathrm{f}=$ hatchi_s0( $t$, Iter, J, N, mcoef, lambda );

\section{Descrição}

A função hatchi_s 0 tem seis argumentos de entrada

i. t é um vetor coluna $\left(t_{1}, \ldots, t_{m}\right)^{\top}$ que desejamos calcular $\chi_{s_{0}}\left(t_{j}\right), j=1, \ldots, m$

ii. Iter é um número inteiro positivo tal que $\frac{1}{2^{\text {Iter }}}$ é a precisão utilizada nos métodos de aproximação para integrais envolvendo a função escala $\phi(x)$ 
iii. J é o nível de aproximação

iv. N é um número inteiro positivo indexando a ordem na família Daubechies

v. mcoef é uma matriz cuja i-ésima coluna é composta pelos coeficientes da aproximação da curva $\chi_{\boldsymbol{s}_{i}}(t)$

vi. lamb da é um vetor coluna constituido pelos parâmetros $\lambda_{1}, \ldots, \lambda_{n}$ no modelo de Krigagem Ordinária Funcional

e retorna um vetor coluna $f=\left(\chi_{\boldsymbol{s}_{0}}\left(t_{1}\right), \ldots, \chi_{\boldsymbol{s}_{0}}\left(t_{m}\right)\right)^{\top}$ com o mesmo número de colunas de $t$.

\section{Código Fonte}

Listing A.16: Arquivo hatchi_s0.m.

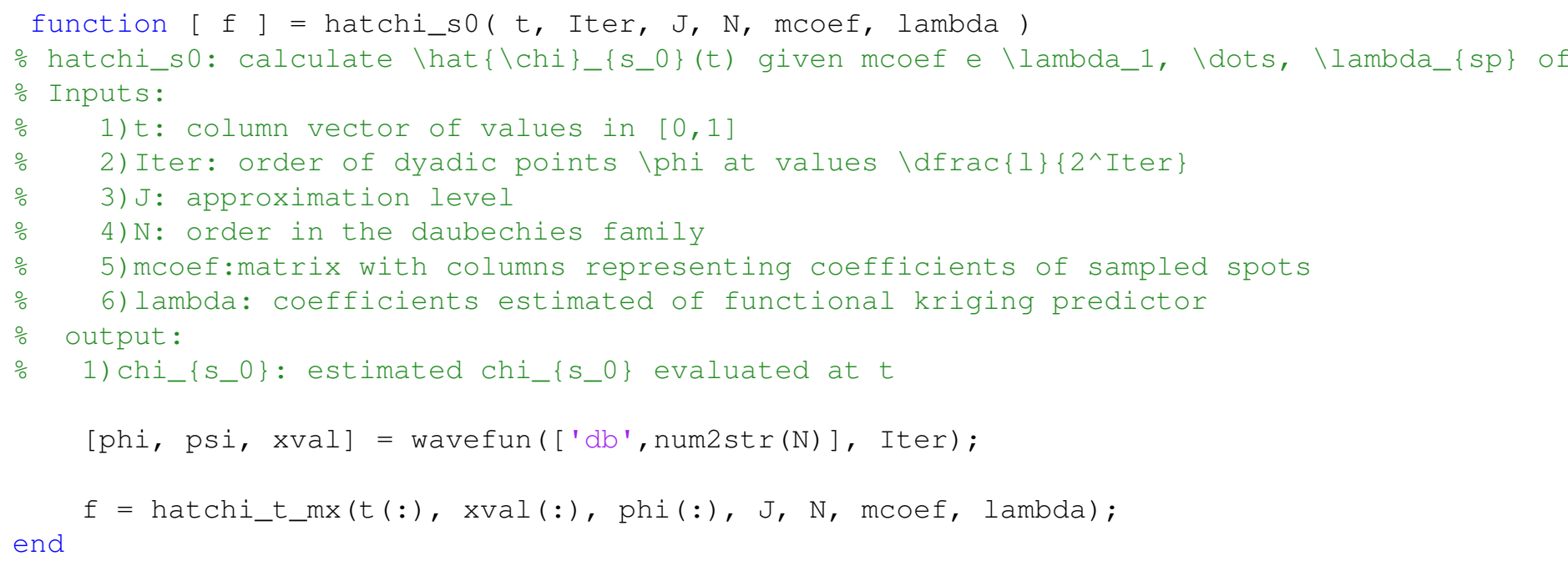

Note que a função hatchi_so usa a função hatchi_t_mx que foi implementada usando MEX file cujo código hatchi_t_mx.c fonte é transcrito em Listing A.17.

Listing A.17: Arquivo hatchi_t_mx.c.

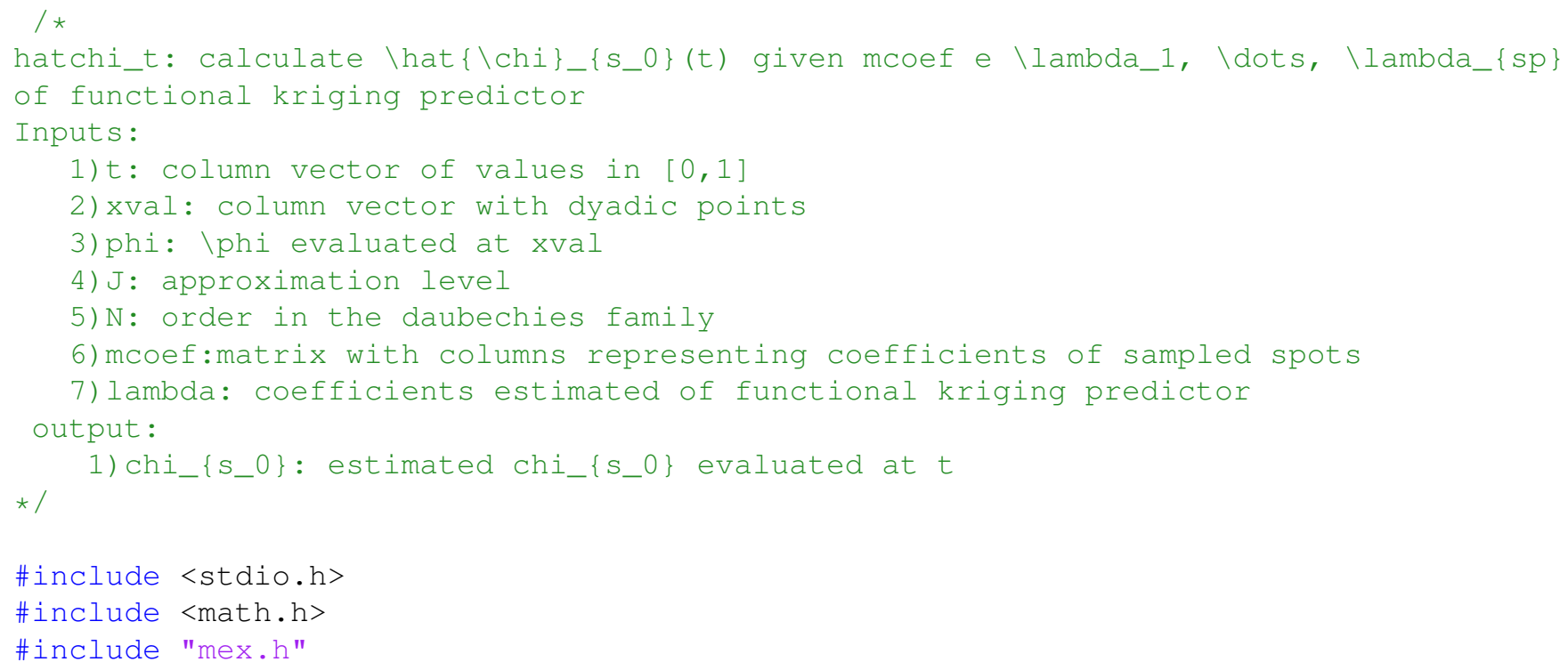




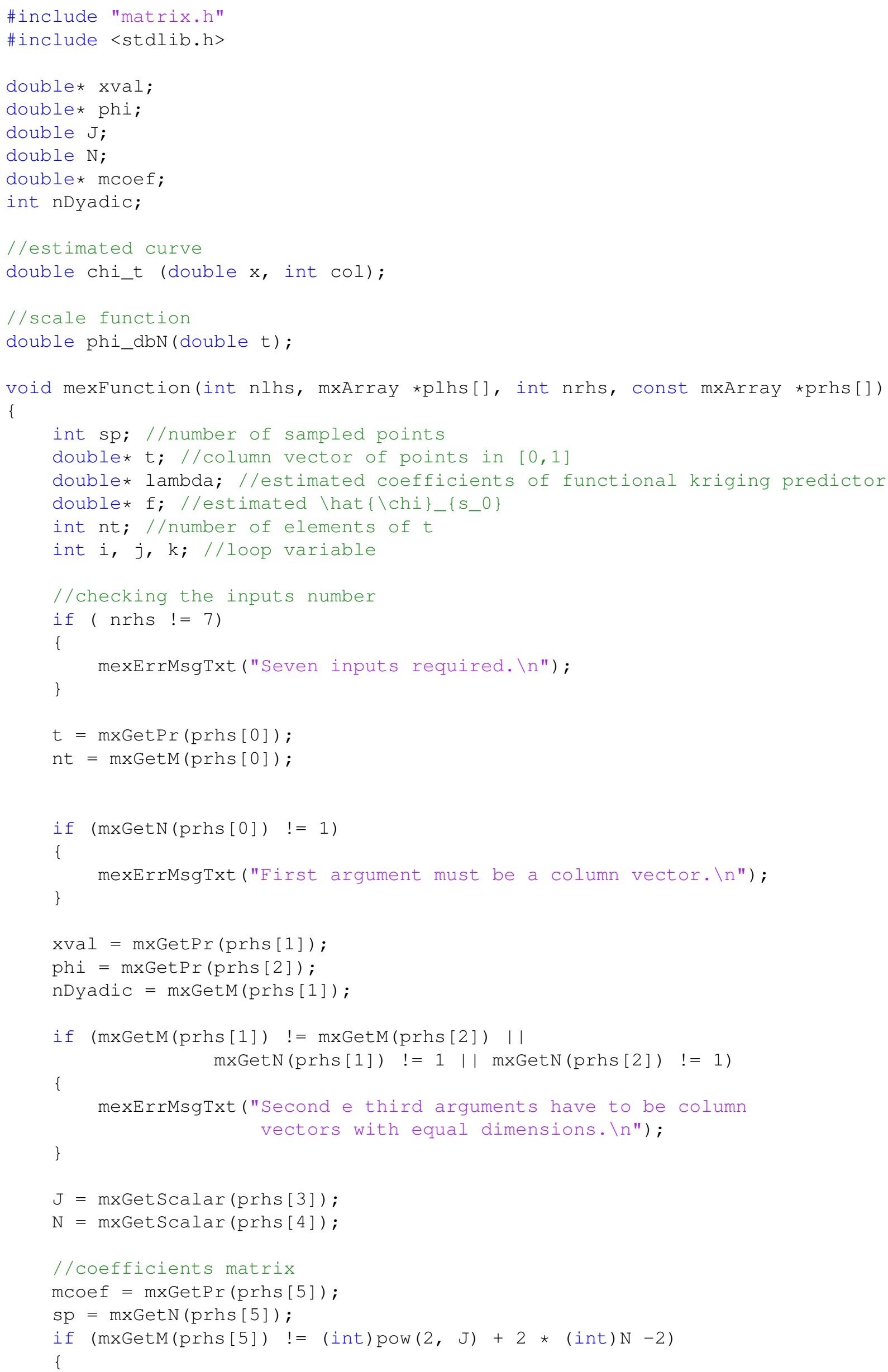




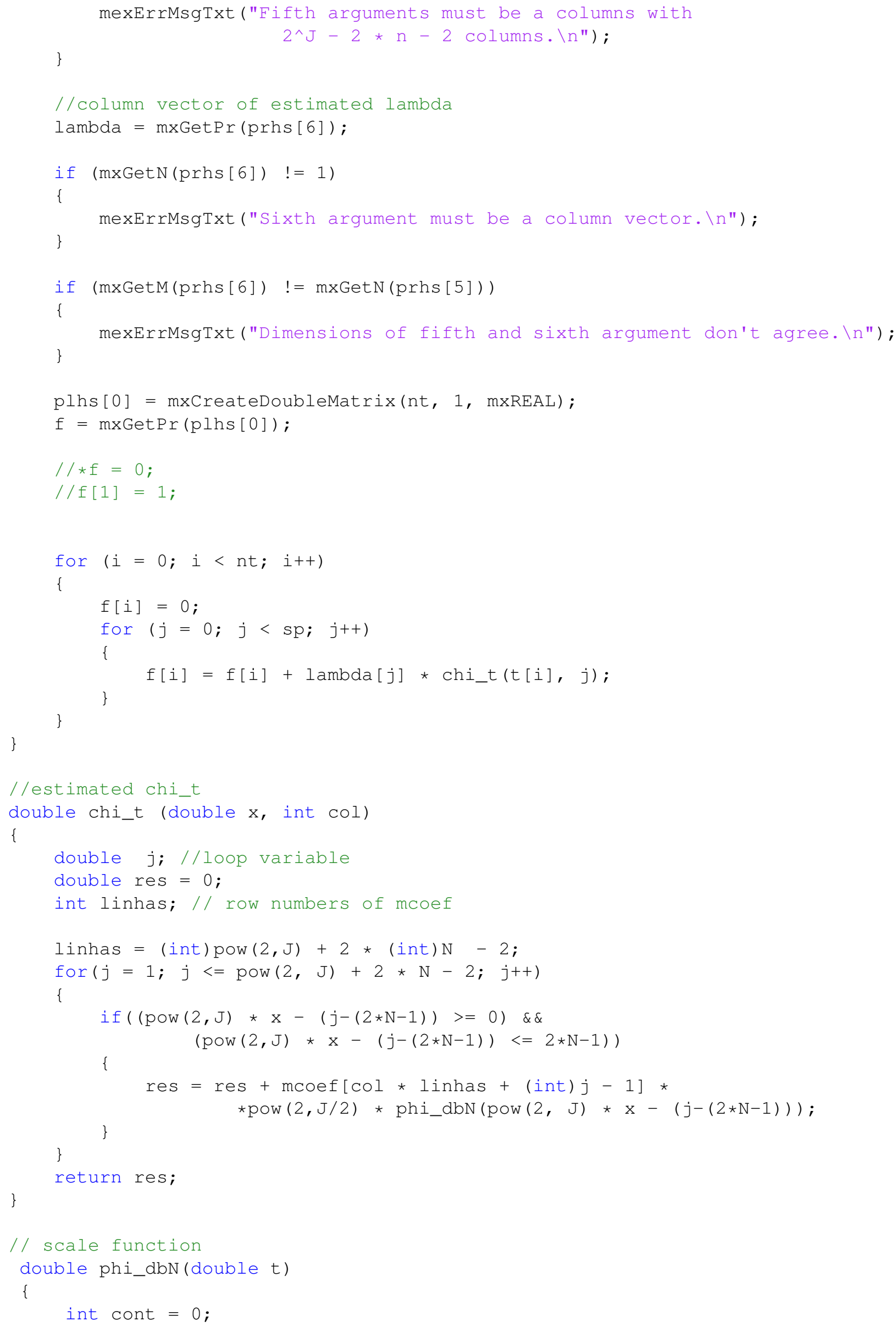




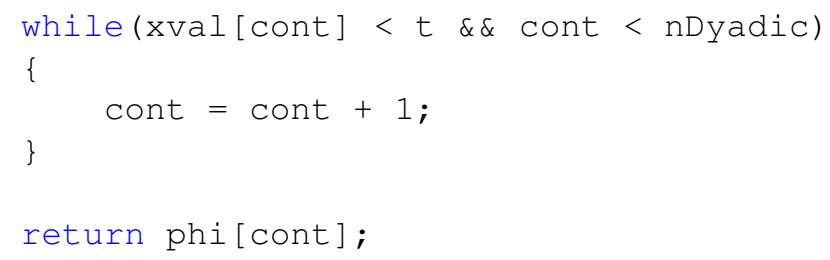

\section{A.15 PointwiseFuncKriging_dbN}

\section{Objetivo}

Dada uma amostra funcional $\chi_{\boldsymbol{s}_{1}}(t), \ldots, \chi_{\boldsymbol{s}_{n}}(t)$, esta função estima uma curva $\chi_{\boldsymbol{s}_{0}}(t)$ em um ponto $\boldsymbol{s}_{0} \notin\left\{\boldsymbol{s}_{1}, \ldots, \boldsymbol{s}_{n}\right\}$ usando o modelo de Krigagem Tempo-Variante Funcional conforme descrito na Seção 3.2 para ondaletas da família Daubechies.

\section{Sintaxe}

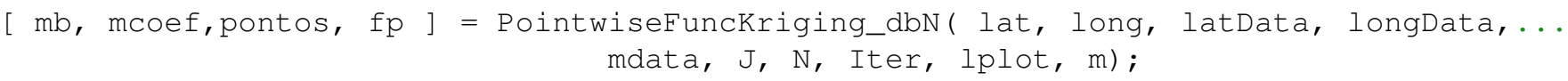

\section{Descrição}

A função PointwiseFuncKriging_dbN tem dez argumentos de entrada

i. lat é a latitude do ponto não monitorado $s_{0}$

ii. long é a longitude do ponto não monitorado $s_{0}$

iii. LatData é um vetor coluna contendo as latitudes dos pontos $\boldsymbol{s}_{1}, \ldots, \boldsymbol{s}_{n}$ das curvas em nossa amostra funcional

iv. longData é um vetor coluna contendo as longitudes dos pontos $s_{1}, \ldots, s_{n}$ das curvas em nossa amostra funcional

v. mdata é um matriz em que a i-ésima coluna é a série temporal $\chi_{\boldsymbol{s}_{i}}\left(t_{1}\right), \ldots, \chi_{\boldsymbol{s}_{i}}\left(t_{n}\right)$ observada da curva $\chi_{\boldsymbol{s}_{i}}(t)$

vi. J é o nível de aproximação na análise de multirresolução

vii. N é um número inteiro positivo que indexa a ordem na família Daubechies

viii. Iter é uma número inteiro positivo tal que $\frac{1}{2^{\text {Iter }}}$ precisão numéricas para aproximação de integrais envolvendo a função escala $\phi(x)$. Este argumento é opcional e seu valor padrão é 10.

ix. lplot é uma variável lógica. Se true, a função produz um gráfico com a curva estimada. Esta argumento é opcional e seu valor padrão é true.

x. m é um número inteiro positivo usado para determinar o número de pontos usados nas abscissas para construir o gráfico da curva estimada caso lplot seja true

e retorna quatro saídas 
a. mb é uma matriz cuja i-ésima coluna contém o vetor de parâmetros $\boldsymbol{b}_{i}$ do modelo de Krigagem Tempo-Variante Funcional descrito na Seção 3.2

b. mcoef é uma matriz cuja i-ésima coluna contém os coeficientes da curva $\chi_{\boldsymbol{s}_{i}}(t)$

c. pontos é um vetor coluna $\left(t_{1}, \ldots, t_{m}\right)^{\top}$ com m pontos entre 0 e 1

d. fp é vetor coluna $\left(\chi_{\boldsymbol{s}_{0}}\left(t_{1}\right), \ldots, \chi_{\boldsymbol{s}_{0}}(t)\right)^{\top}$ com o mesmo número de linhas de $t$ contendo as estimativas da curva $\chi_{\boldsymbol{s}_{0}}(t)$ no ponto não monitorado $\boldsymbol{s}_{0}$ pelo método de Krigagem TempoVariante Funcional

\section{Código Fonte}

Listing A.18: Arquivo PointwiseFuncKriging_dbN.

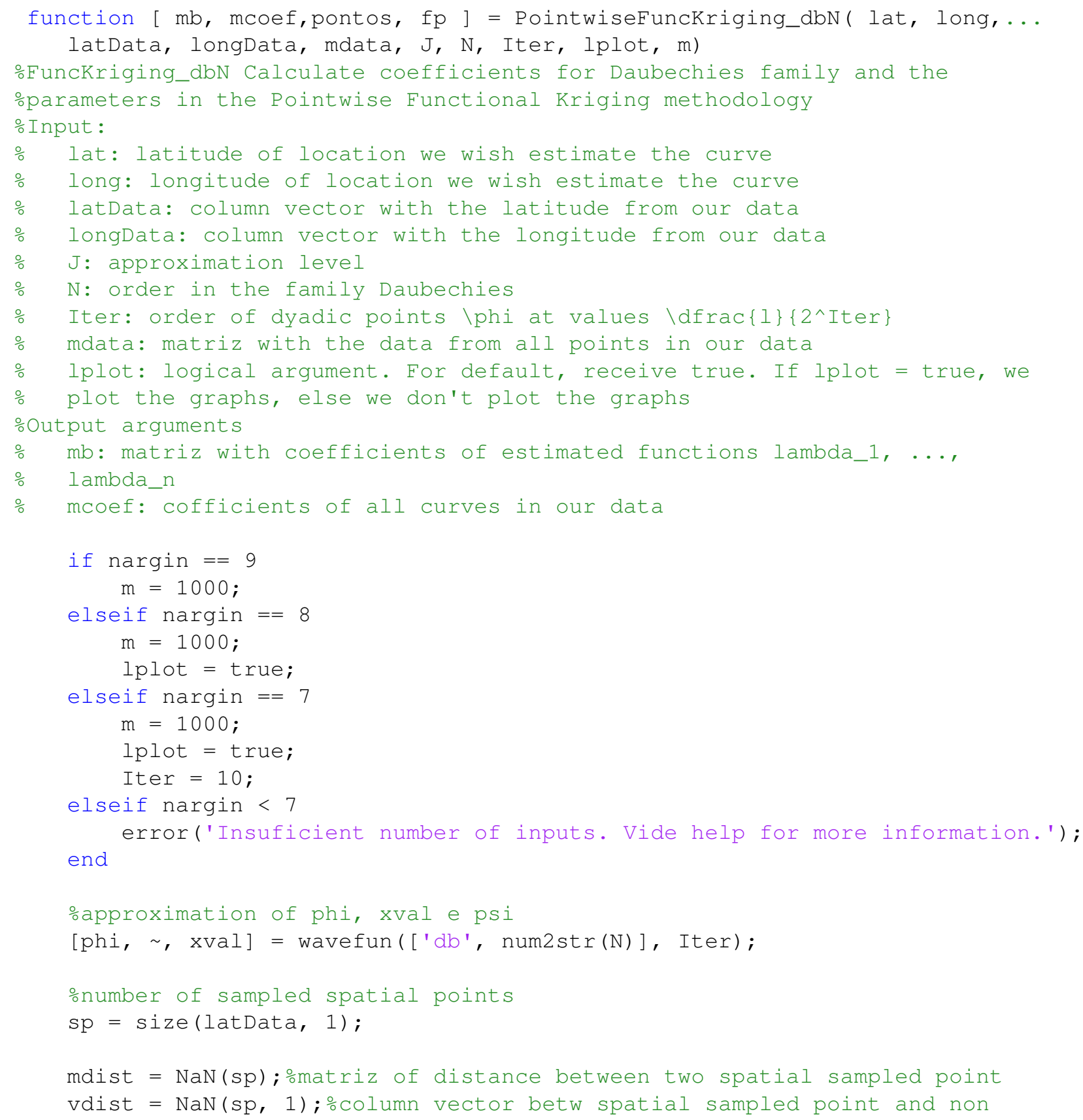


\% sampled point

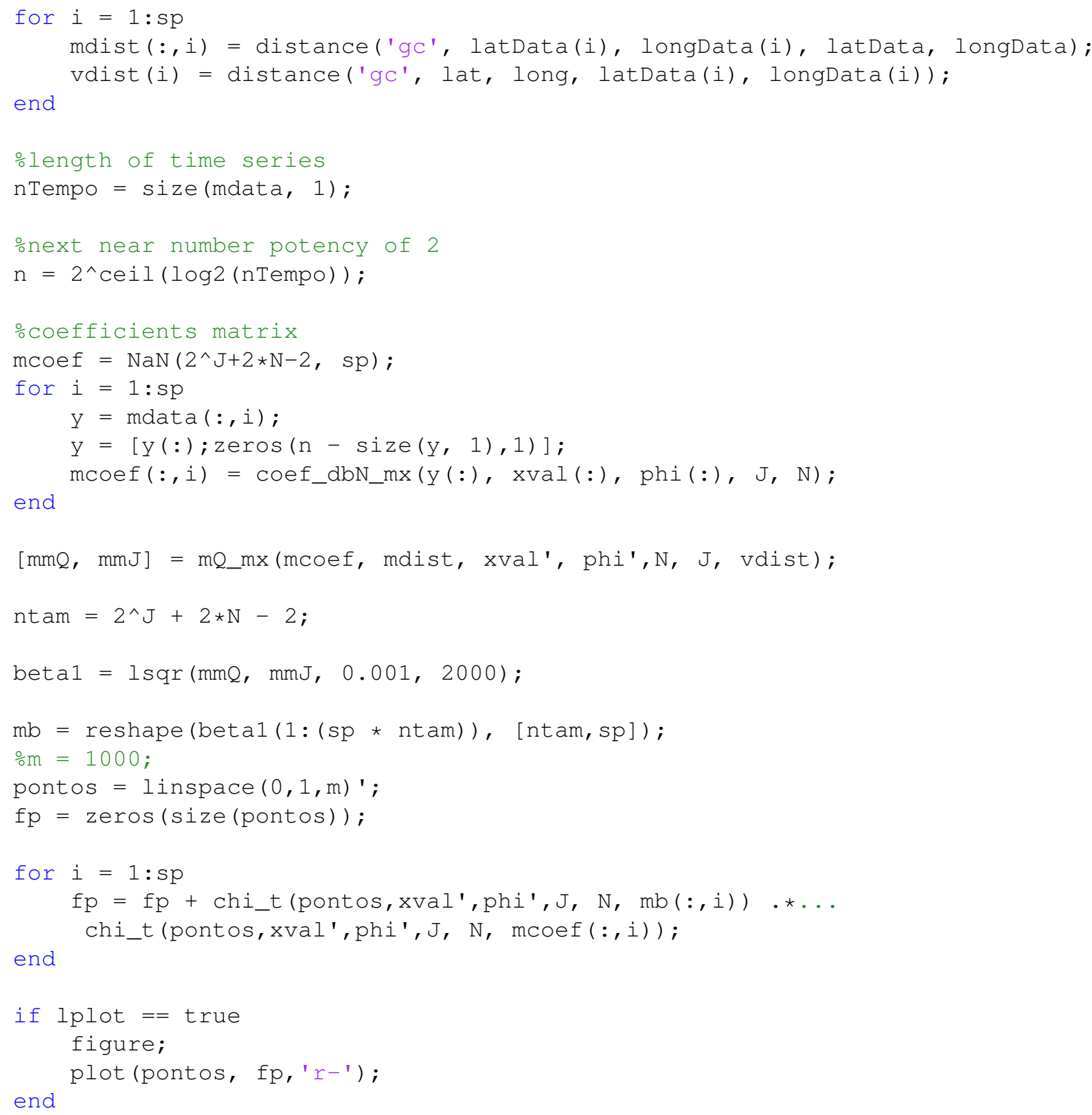

A função PointwiseFuncKriging_dbN usa a função mQ_mx que foi implementada usando MEX file cujo código fonte é transcrito a seguir.

Listing A.19: Arquivo $m Q \_m x . c$.

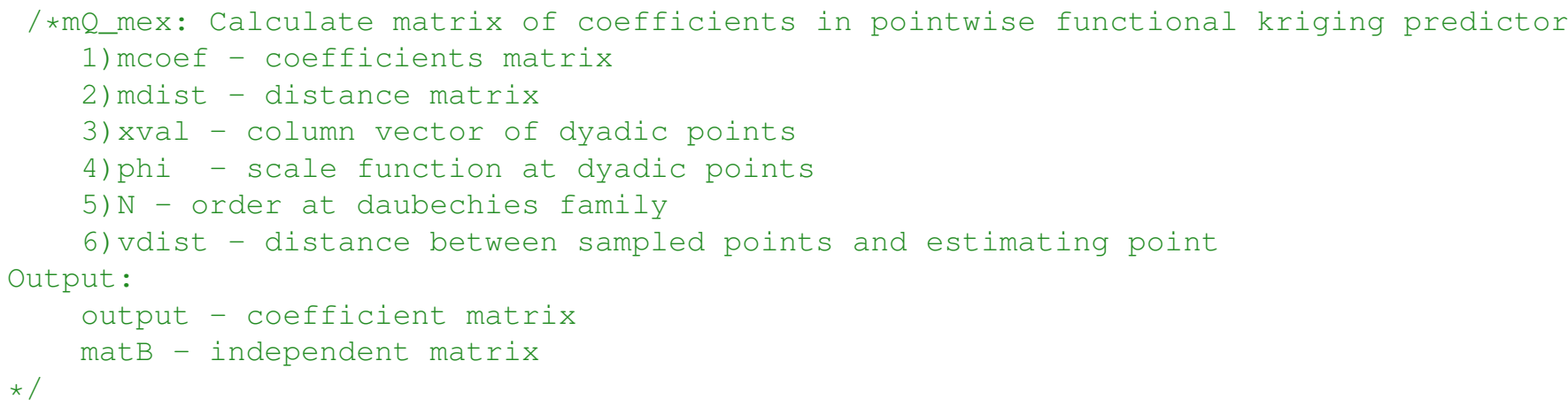




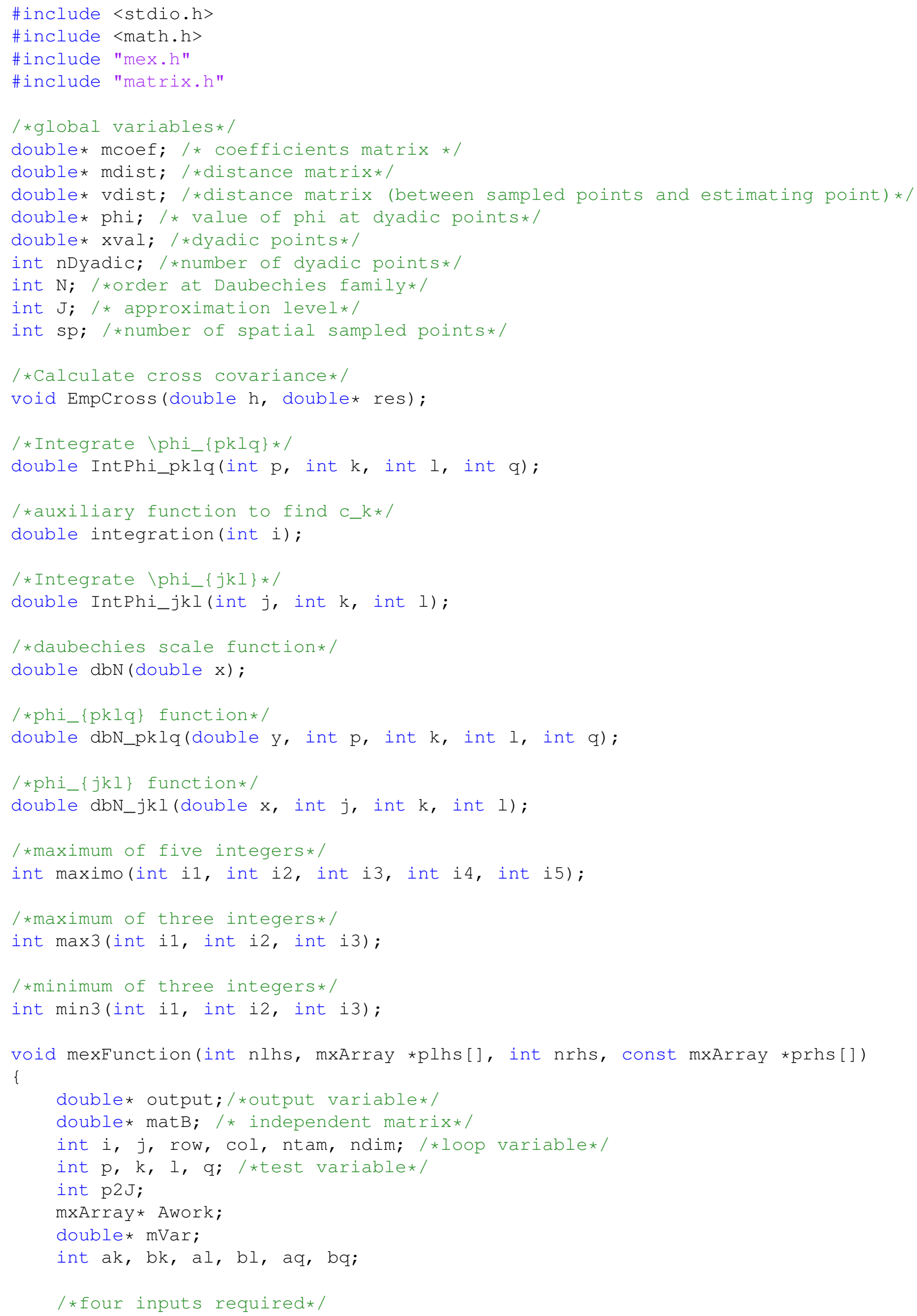




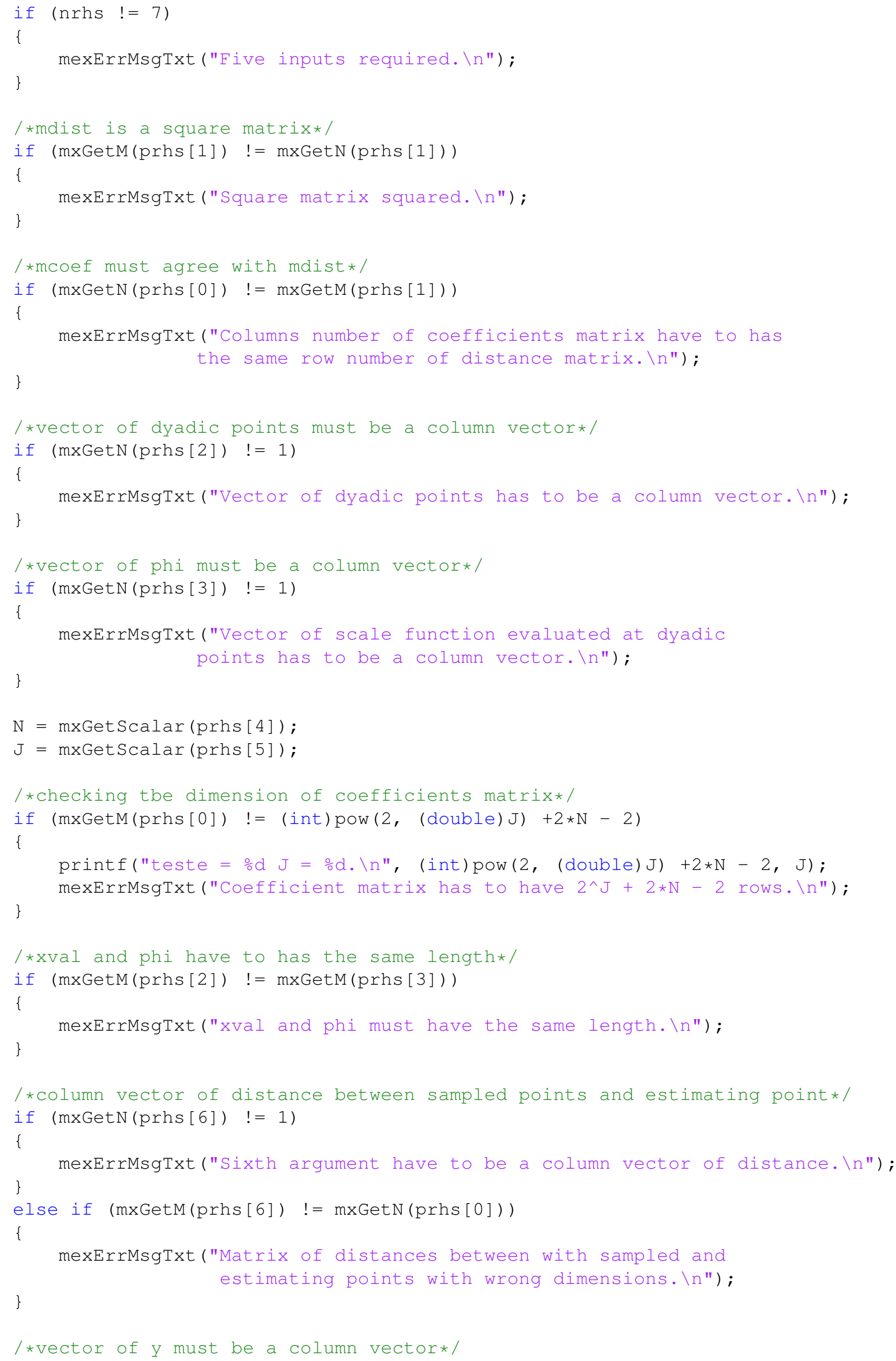




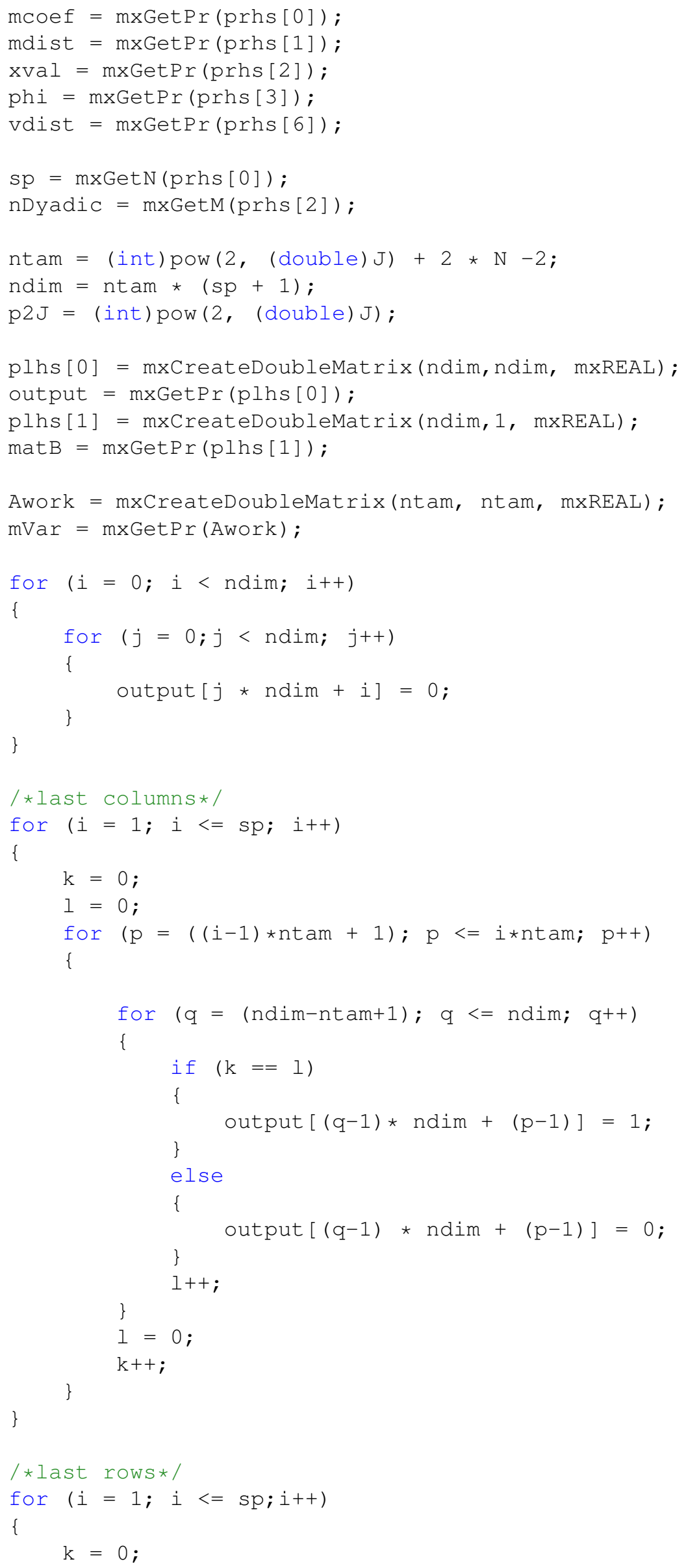




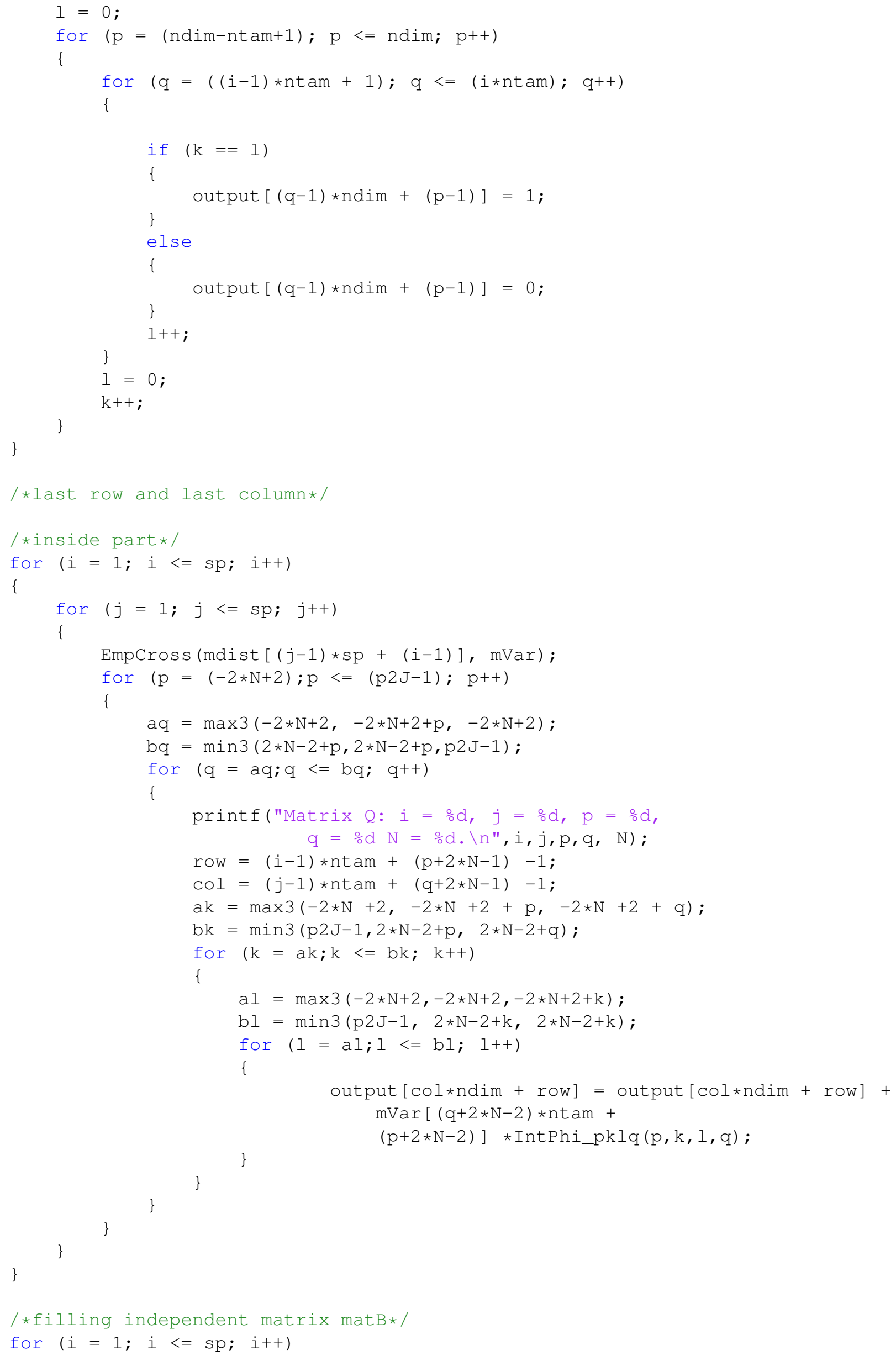


\{

EmpCross (voist $[i-1], \operatorname{mVar})$;

for $(j=-2 \star N+2 ; j<=p 2 J-1 ; j++)$

\{

printf("matrix $\left.b: i=\frac{o}{d} j=\frac{\circ}{d} \mathrm{~N}=\frac{\circ}{\mathrm{d}} . \backslash \mathrm{n} n, \boldsymbol{i}, j, \mathrm{~N}\right)$;

$\operatorname{mat} B[(\mathrm{i}-1) \star \mathrm{ntam}+(\mathrm{j}+2 * \mathrm{~N}-2)]=0$;

$\mathrm{ak}=\max 3(-2 \star \mathrm{N}+2+j,-2 \star \mathrm{N}+2,-2 \star \mathrm{N}+2)$;

$\mathrm{bk}=\min 3(\mathrm{p} 2 \mathrm{~J}-1,2 \star \mathrm{N}-2+\mathrm{j}, 2 \star \mathrm{N}-2+\mathrm{j}) ;$

for $(\mathrm{k}=\mathrm{ak} ; \mathrm{k}<=\mathrm{bk} ; \mathrm{k}++)$

\{

al $=\max 3(-2 * \mathrm{~N}+2,-2 * \mathrm{~N}+2+\mathrm{k},-2 * \mathrm{~N}+2+\mathrm{j}) ;$

$\mathrm{bl}=\min 3(\mathrm{p} 2 \mathrm{~J}-1,2 * \mathrm{~N}-2+\mathrm{j}, 2 * \mathrm{~N}-2+\mathrm{k})$;

for $(l=a l ; l<=b l ; l++)$

\{

$\operatorname{mat} B[(i-1) * n \operatorname{tam}+(j+2 * \mathrm{~N}-2)]=\operatorname{matB}[(i-1) * \mathrm{ntam}+(j+2 * \mathrm{~N}-2)]+$ $\operatorname{mVar}[(\mathrm{k}+2 * \mathrm{~N}-2) * \mathrm{ntam}+(1+2 * \mathrm{~N}-2)] * \operatorname{IntPhi} j \mathrm{kl} l(j, \mathrm{k}, 1)$;

\}

\}

\}

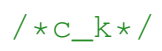




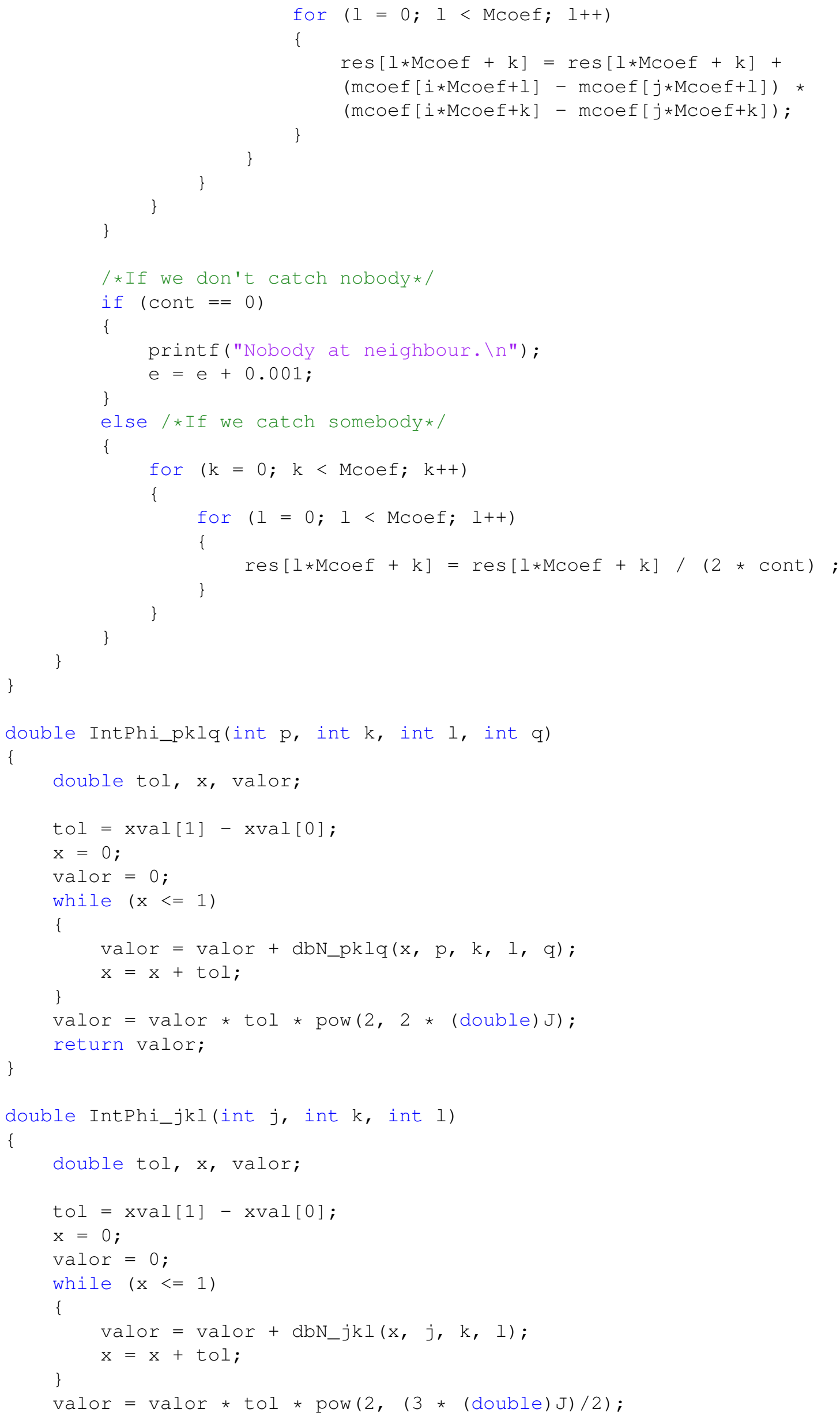




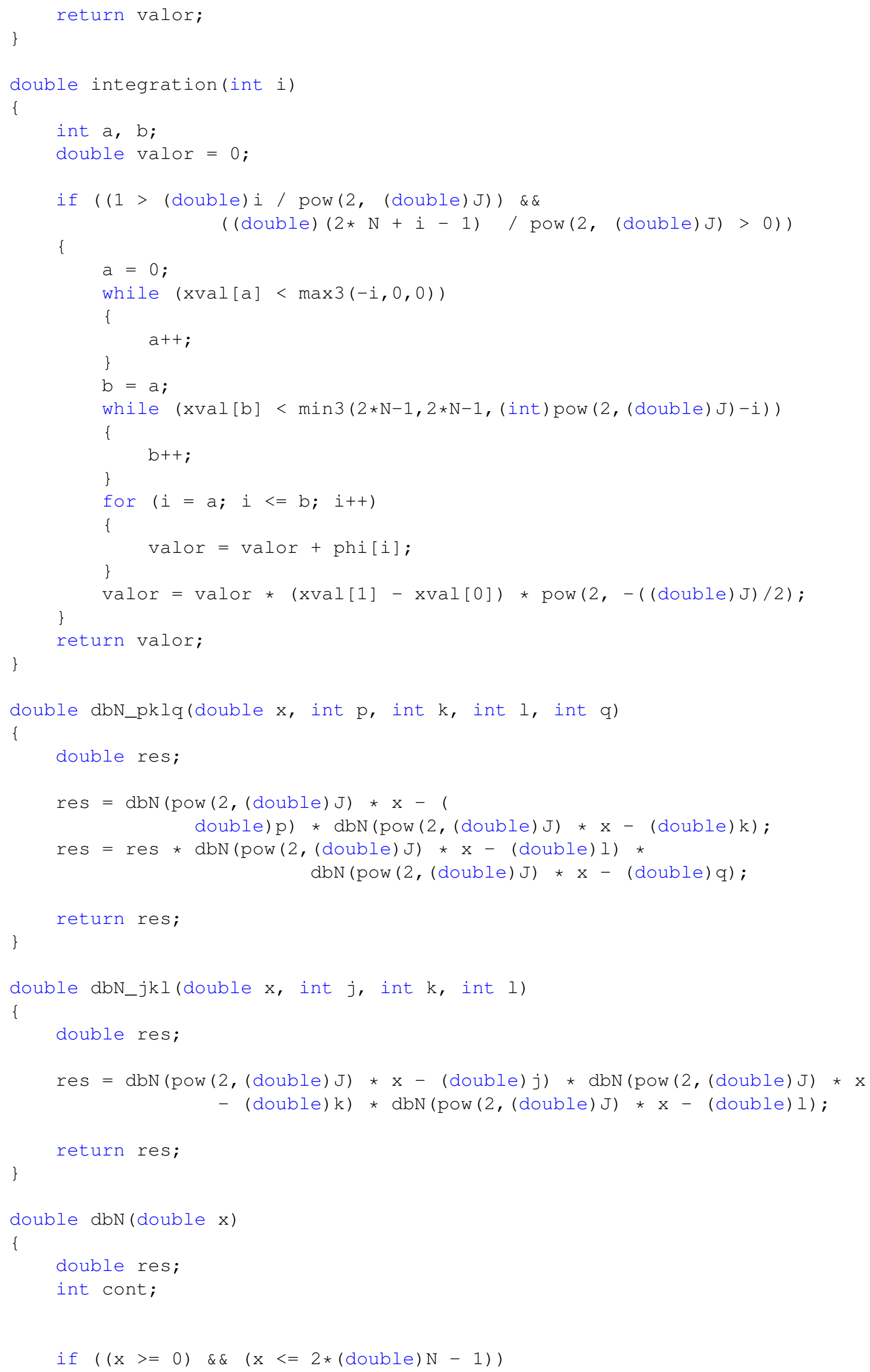




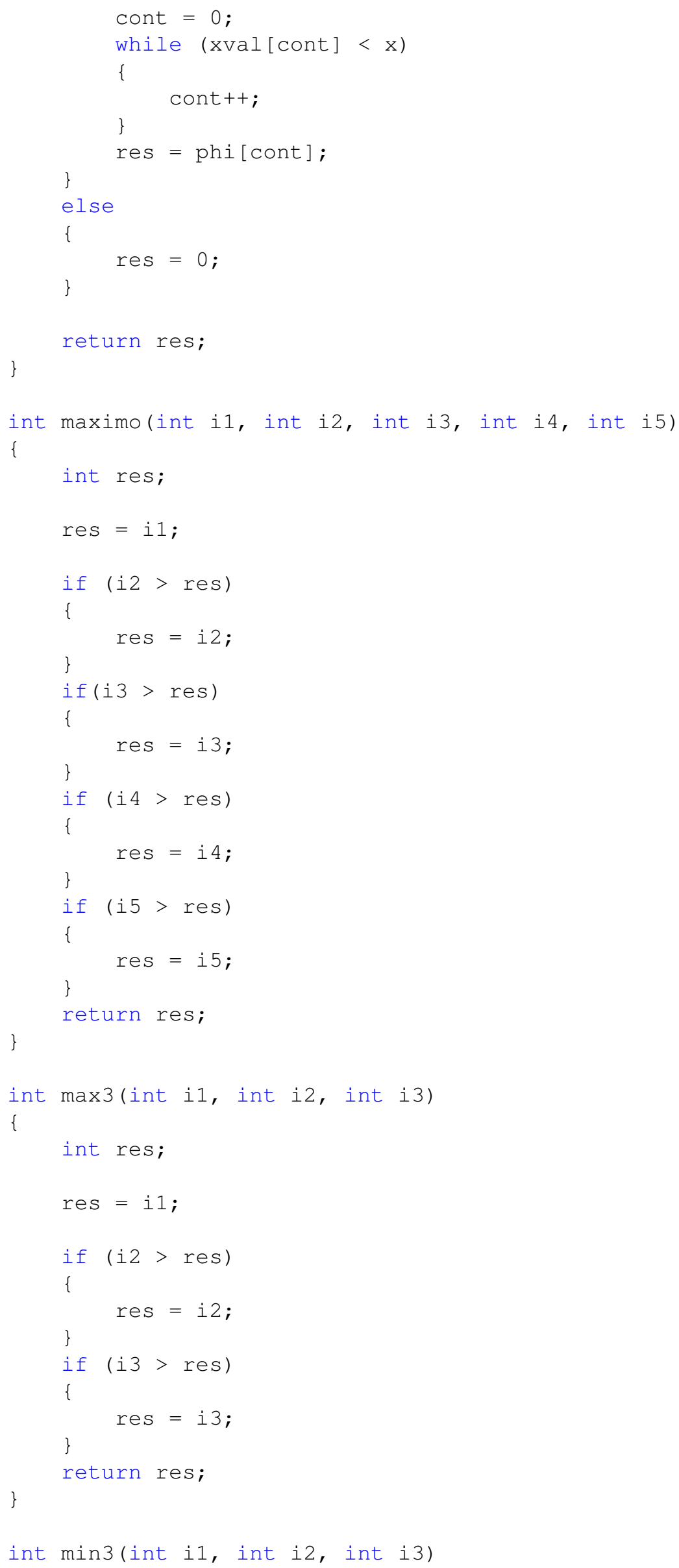


\{

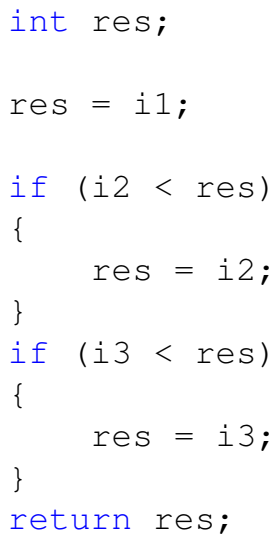

\section{A.16 hatchi}

\section{Objetivo}

Esta função computa o valor da curva $\chi_{\boldsymbol{s}_{0}}(t)$ no ponto $t$, quando os vetores $\boldsymbol{b}_{i}$ do modelo de Krigagem Tempo-Variante Funcional são conhecido ou estimados e base de funções utilizadas são as ondaletas da família Daubechies.

\section{Sintaxe}

$\mathrm{fp}=\operatorname{hatchi}(t$, Iter, $\mathrm{J}, \mathrm{N}, \operatorname{mcoef}, \mathrm{mb})$

\section{Descrição}

A função hatchi tem seis argumentos de entrada

i. t é um vetor coluna $\left(t_{1}, \ldots, t_{m}\right)^{\top} \operatorname{com} t_{j} \in[0,1]$

ii. Iter é um número inteiro positivo em que $\frac{1}{2^{\text {Iter }}}$ é a precisão utilizada nas aproximações numéricas de integrais envolvendo a função escala $\phi(x)$

iii. J é o nível de aproximação

iv. N é um número inteiro positivo indexando a família Daubechies

v. mcoef é uma matriz em que a i-ésima coluna contém os coeficientes da curva $\chi_{\boldsymbol{s}_{i}}(t)$

vi. mb é uma matriz em que a i-ésima coluna consiste do vetor $\boldsymbol{b}_{i}$ que é o vetor de coeficientes da função $\lambda_{i}(t)$ no modelod de Krigagem Tempo-Variante Funcional

e retorna um vetor coluna $\left(\chi_{s_{0}}\left(t_{1}\right), \ldots, \chi_{s_{0}}\left(t_{m}\right)\right)^{\top}$ com o mesmo número de linhas do vetor $t$.

\section{Código Fonte}


Listing A.20: Arquivo hatchi . c.

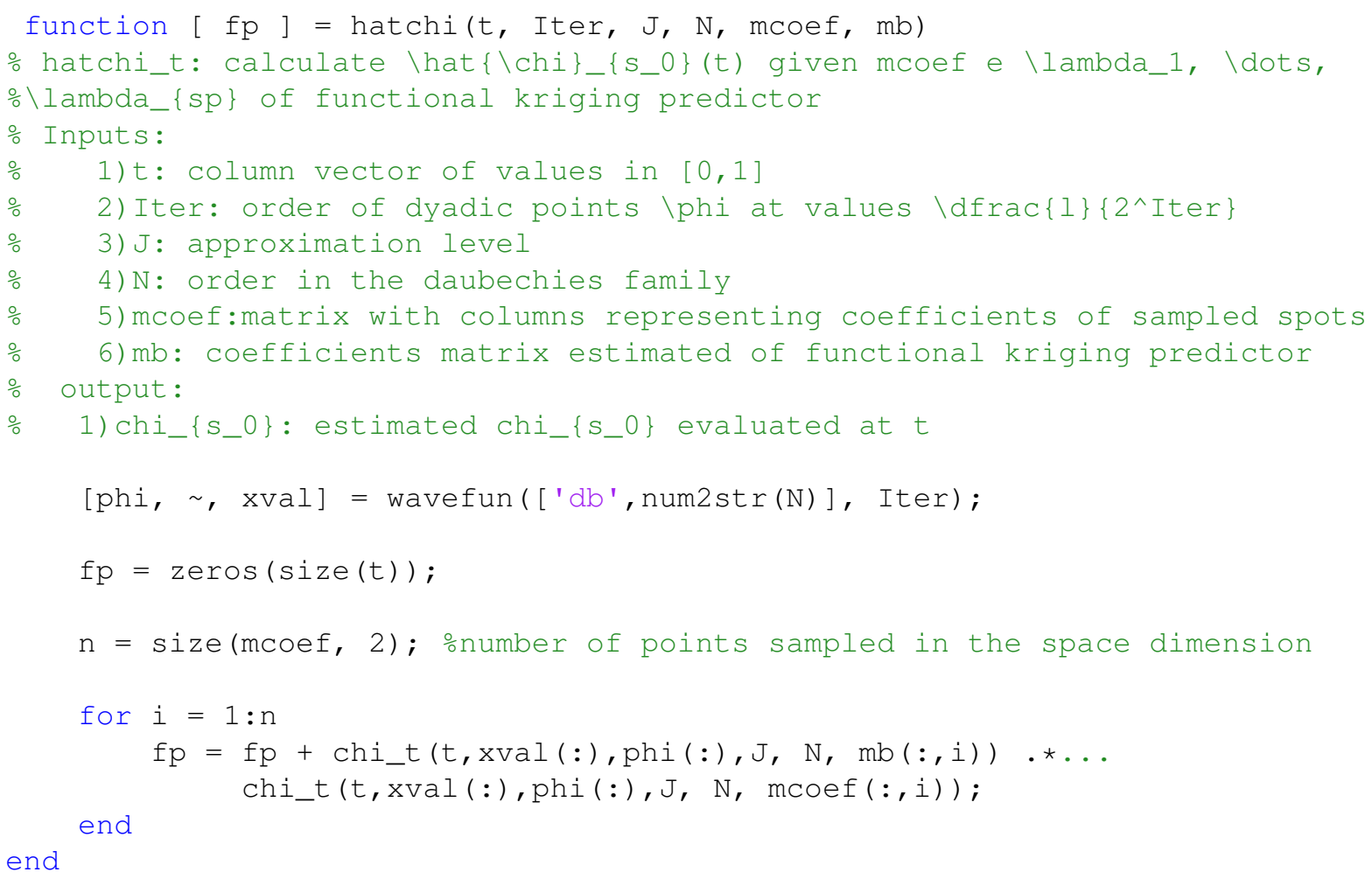




\section{Apêndice B}

\section{Estações Meteorológicas - Províncias Marítimas Canadenses}

Na Tabela B.1, mostramos as informações referentes as 82 estaçoes meteorológicas selecionadas nas províncias marítimas. Incluímos nessa tabela quatro informações

ID Número usado pela agência canadense Environment and Climate Change Canada (ECCC) para identificar as estações meteorológicas;

Nome Nome da estação;

Latitude Latitude da localização da estação;

Longitude Longitude da localização da estação.

\begin{tabular}{clcc}
\hline ID & Nome & latitude & longitude \\
\hline 6106 & ACADIA FOREST EXP ST & 45.99 & -66.36 \\
6108 & ALMA & 45.60 & -64.95 \\
6109 & AROOSTOOK & 46.71 & -67.72 \\
6915 & BAS CARAQUET & 47.80 & -64.83 \\
6916 & BATHURST A & 47.63 & -65.75 \\
6119 & BERTRAND & 47.75 & -65.07 \\
6137 & CHARLO A & 47.98 & -66.33 \\
6146 & COLESON COVE & 45.15 & -66.20 \\
6150 & DOAKTOWN & 46.55 & -66.14 \\
6157 & FREDERICTON A & 45.87 & -66.53 \\
10981 & FREDERICTON AQUATIC CENTRE C/S & 45.96 & -66.65 \\
6160 & GAGETOWN 2 & 45.78 & -66.15 \\
6170 & HARCOURT & 46.50 & -65.27 \\
27482 & HARVEY STATION & 45.73 & -67.02 \\
6175 & HAUT SHIPPAGAN & 47.75 & -64.77 \\
26970 & HAVELOCK & 46.00 & -65.32 \\
6180 & HOYT BLISSVILLE & 45.60 & -66.57 \\
6181 & JUNIPER & 46.55 & -67.17 \\
26968 & KOUCHIBOUGUAC CS & 46.77 & -65.01 \\
6194 & MACTAQUAC PROV PARK & 45.95 & -66.90 \\
6197 & MAPLETON & 46.18 & -67.23 \\
6140 & MIRAMICHI A & 47.01 & -65.47
\end{tabular}




\begin{tabular}{|c|c|c|c|}
\hline 6206 & MONCTON & 46.10 & -64.79 \\
\hline 6207 & MONCTON A & 46.11 & -64.68 \\
\hline 6208 & MOUNT CARLETON & 47.42 & -66.93 \\
\hline 6212 & NAUWIGEWAUK & 45.47 & -65.90 \\
\hline 6213 & NEPISIGUIT FALLS & 47.40 & -65.78 \\
\hline 6215 & NICTAU & 47.23 & -67.15 \\
\hline 6219 & PARKINDALE & 45.87 & -65.07 \\
\hline 6220 & PENNFIELD & 45.10 & -66.73 \\
\hline 6232 & REXTON & 46.67 & -64.87 \\
\hline 6241 & SACKVILLE & 45.85 & -64.38 \\
\hline 6250 & SAINT JOHN A & 45.32 & -65.89 \\
\hline 6256 & ST LEONARD A & 47.16 & -67.83 \\
\hline 6268 & SUSSEX & 45.72 & -65.53 \\
\hline 6280 & UPSALQUITCH LAKE & 47.46 & -66.42 \\
\hline 6920 & WOLFE LAKE CS & 45.67 & -65.15 \\
\hline 6921 & AVONDALE & 45.02 & -64.12 \\
\hline 27602 & BERRYS BAY & 43.66 & -65.26 \\
\hline 6308 & BRIDGEWATER & 44.40 & -64.55 \\
\hline 6318 & CHARLESVILLE & 43.58 & -65.78 \\
\hline 27600 & CHETICAMP CS & 46.65 & -60.95 \\
\hline 6329 & COLLEGEVILLE & 45.48 & -62.02 \\
\hline 6334 & DEBERT & 45.42 & -63.42 \\
\hline 6336 & DEMING & 45.22 & -61.18 \\
\hline 6354 & GREENWOOD A & 44.98 & -64.92 \\
\hline 6357 & HALIFAX CITADEL & 44.65 & -63.58 \\
\hline 6358 & HALIFAX STANFIELD INT'L A & 44.88 & -63.50 \\
\hline 6370 & JACKSON & 45.58 & -63.83 \\
\hline 6923 & KEJIMKUJIK 1 & 44.40 & -65.20 \\
\hline 27742 & LAKE MAJOR & 44.72 & -63.48 \\
\hline 6383 & LIVERPOOL BIG FALLS & 44.13 & -64.93 \\
\hline 6388 & LOUISBOURG & 45.90 & -60.00 \\
\hline 6393 & LYONS BROOK & 45.66 & -62.80 \\
\hline 6409 & MIDDLE MUSQUODOBOIT & 45.07 & -63.10 \\
\hline 6407 & MIDDLEBORO & 45.77 & -63.57 \\
\hline 6414 & NAPPAN CDA & 45.77 & -64.25 \\
\hline 6428 & PARRSBORO & 45.40 & -64.33 \\
\hline 6435 & POCKWOCK LAKE & 44.77 & -63.83 \\
\hline 6437 & POINT ACONI & 46.32 & -60.33 \\
\hline 6447 & PUGWASH & 45.84 & -63.66 \\
\hline 6465 & SHEARWATER A & 44.63 & -63.50 \\
\hline 26969 & SOUTH MOUNTAIN & 45.02 & -64.68 \\
\hline 27282 & SOUTH SIDE HARBOUR & 45.62 & -61.90 \\
\hline 6473 & SPRINGFIELD & 44.67 & -64.85 \\
\hline 6456 & ST MARGARET'S BAY & 44.70 & -63.90 \\
\hline 6484 & SUMMERVILLE & 45.12 & -64.18 \\
\hline 6486 & SYDNEY A & 46.17 & -60.05 \\
\hline 6927 & TATAMAGOUCHE & 45.68 & -63.23 \\
\hline 27868 & TRENTON MUNICIPAL A & 45.61 & -62.62 \\
\hline 6497 & WATERVILLE CAMBRIDGE & 45.05 & -64.65 \\
\hline
\end{tabular}




\begin{tabular}{ll}
6512 & WINDSOR MARTOCK \\
6514 & WRECK COVE BROOK \\
6516 & YARMOUTH A \\
6519 & ALBERTON \\
6520 & ALLISTON \\
6522 & BANGOR \\
6929 & ELMWOOD \\
6538 & LONG RIVER \\
6536 & MONTICELLO \\
6540 & O'LEARY \\
6931 & VICTORIA \\
\hline
\end{tabular}

$\begin{array}{ll}44.93 & -64.17 \\ 46.53 & -60.45 \\ 43.83 & -66.09 \\ 46.85 & -64.02 \\ 46.07 & -62.60 \\ 46.35 & -62.68 \\ 46.25 & -63.33 \\ 46.50 & -63.55 \\ 46.47 & -62.47 \\ 46.70 & -64.26 \\ 46.22 & -63.49\end{array}$

Tabela B.1: Tabela com as informações das estações meteorológicas selecionadas nas províncias marítimas canadenses. 
APÊNDICE B 


\section{Apêndice C}

\section{Estações Meteorológicas - Região Metropolitana de São Paulo}

Na Tabela C.1, mostramos as informações referentes as 14 estaçoes meteorológicas selecionadas na região metropolitana de São Paulo mantidas pela CETESB (Companhia Ambiental do Estado De São Paulo). Incluímos nessa tabela quatro informações

ID Número usado pela CETESB para identificar as estações meteorológicas;

Nome Nome da estação;

Latitude Latitude da localização da estação;

Longitude Longitude da localização da estação.

Tabela C.1: Tabela com as informações das estações meteorológicas da CETESB selecionadas na Região Metropolitana de São Paulo.

\begin{tabular}{lccc}
\hline Nome & ID & Latitude & Longitude \\
\hline Santana & 63 & -23.5151 & -46.6293 \\
Santo Amaro & 64 & -23.6546 & -46.7096 \\
Pq Dom Pedro II & 72 & -23.544 & -46.6277 \\
Congonhas & 73 & -23.6158 & -46.6633 \\
Mooca & 85 & -23.5477 & -46.6031 \\
São Caetano & 86 & -23.6182 & -46.5563 \\
Diadema & 92 & -23.6881 & -46.6133 \\
Nossa Senhora do Ó & 96 & -23.4798 & -46.6924 \\
Osasco & 120 & -23.5266 & -46.7923 \\
Sto André - Pço Municipal & 254 & -23.655 & -46.5316 \\
Carapicuiba & 263 & -23.5301 & -46.8357 \\
Guarulhos - Pço Municipal & 264 & -23.4559 & -46.5184 \\
Itaim Paulista & 266 & -23.5017 & -46.4204 \\
Pte Remédios & 270 & -23.5092 & -46.7095 \\
\hline
\end{tabular}


APÊNDICE C 


\section{Referências Bibliográficas}

Banerjee et al.(2008) Sudipto Banerjee, Alan E Gelfand, Andrew O Finley e Huiyan Sang. Gaussian predictive process models for large spatial data sets. Journal of the Royal Statistical Society: Series B (Statistical Methodology), 70(4):825-848. Citado na pág. 66

Banerjee et al.(2014) Sudipto Banerjee, Bradley P Carlin e Alan E Gelfand. Hierarchical modeling and analysis for spatial data. Crc Press. Citado na pág. 7, 22, 41, 42, 65

Boggess e Narcowich(2009) Albert Boggess e Francis J Narcowich. A first course in wavelets with Fourier analysis. John Wiley \& Sons. Citado na pág. 12, 35

Bohorquez et al.(2015) Martha Bohorquez, Ramón Giraldo e Jorge Mateu. Optimal sampling for spatial prediction of functional data. Statistical Methods \& Applications, páginas 1-16. Citado na pág. 2

Brown et al.(1994) Philip J Brown, Nhu D Le e James V Zidek. Multivariate spatial interpolation and exposure to air pollutants. Canadian Journal of Statistics, 22(4):489-509. Citado na pág. 64

Caballero et al.(2013) William Caballero, Ramón Giraldo e Jorge Mateu. A universal kriging approach for spatial functional data. Stochastic Environmental Research and Risk Assessment, 27(7):1553-1563. Citado na pág. 3

Chiles e Delfiner(2009) Jean-Paul Chiles e Pierre Delfiner. Geostatistics: modeling spatial uncertainty, volume 497. John Wiley \& Sons. Citado na pág. 7

Chui(2014) Charles K Chui. An introduction to wavelets, volume 1. Academic press. Citado na pág. 13

Cressie e Johannesson(2008) Noel Cressie e Gardar Johannesson. Fixed rank kriging for very large spatial data sets. Journal of the Royal Statistical Society: Series B (Statistical Methodology), 70(1):209-226. Citado na pág. 66

Cressie e Cassie(1993) Noel AC Cressie e Noel A Cassie. Statistics for spatial data, volume 900. Wiley New York. Citado na pág. 7

Daubechies et al.(1992) Ingrid Daubechies et al. Ten lectures on wavelets, volume 61. SIAM. Citado na pág. 12,16 
De Oliveira et al.(1997) Victor De Oliveira, Benjamin Kedem e David A Short. Bayesian prediction of transformed gaussian random fields. Journal of the American Statistical Association, 92 (440):1422-1433. Citado na pág. 64

de Souza e Dias(2010) Camila P. Estevam de Souza e Ronaldo Dias. Introdução à análise de dados funcionais. Associação Brasileira de Estatística, São Paulo - SP. Citado na pág. 5

Delicado et al.(2010) Pedro Delicado, Ramón Giraldo, C Comas e Jorge Mateu. Statistics for spatial functional data: Some recent contributions. Environmetrics, 21(3-4):224-239. Citado na pág. 2

Diggle e Ribeiro(2007) Peter Diggle e Paulo Justiniano Ribeiro. Model-based geostatistics. Springer Science \& Business Media. Citado na pág. 7

Diggle e Ribeiro Jr(2002) Peter J Diggle e Paulo J Ribeiro Jr. Bayesian inference in gaussian model-based geostatistics. Geographical and Environmental Modelling, 6(2):129-146. Citado na pág. 63

Ecker(2003) Mark D Ecker. Geostatistics: past, present and future. Environmetrics. Developed under the auspices of UNESCO. Encyclopedia of Life Support Systems (EOLSS). Ed. by AH El-Shaarawi and J. Jureckova, Eolss Publishers, Oxford, UK. http://www. eolss. net. Citado na pág. 1

Ferraty e Vieu(2006) Frédéric Ferraty e Philippe Vieu. Nonparametric functional data analysis: theory and practice. Springer Science \& Business Media. Citado na pág. 2, 5

Frederick Pearson(1990) II Frederick Pearson. Map ProjectionsTheory and Applications. CRC press. Citado na pág. 7

Fuentes(2001) Montserrat Fuentes. A high frequency kriging approach for non-stationary environmental processes. Environmetrics, 12(5):469-483. Citado na pág. 64

Genton e Kleiber(2015) Marc G. Genton e William Kleiber. Cross-covariance functions for multivariate geostatistics. Statistical Science, 30(2):147-163. Citado na pág. 31, 65

Goovaerts(1997) Pierre Goovaerts. Geostatistics for Natural Resources Evaluation. Oxford University Press. Citado na pág. 1

Goulard e Voltz(1992) Michel Goulard e Marc Voltz. Linear coregionalization model: tools for estimation and choice of cross-variogram matrix. Mathematical Geology, 24(3):269-286. Citado na pág. 31,35

Haar(1909) Kármán Th. v. Haar, A. Zur theorie der spannungszustände in plastischen und sandartigen medien. Nachrichten von der Gesellschaft der Wissenschaften zu Göttingen, Mathematisch-Physikalische Klasse, 1909:204-218. Citado na pág. 15 
Haining(2003) Robert Patrick Haining. Spatial data analysis. Cambridge University Press Cambridge. Citado na pág. 7

Härdle et al.(1998) Wolfgang Härdle, Gerard Kerkyacharian, Alexander Tsybakov e Dominique Picard. Wavelets, approximation, and statistical applications. Springer. Citado na pág. 14, 25

Henao(2009) Ramon Giraldo Henao. Geostatistical analysis of functional data. Tese de Doutorado, Universitat Politècnica de Catalunya, Barcelona. Citado na pág. 2, 45, 63, 64

Henao et al.(2010) Ramon Giraldo Henao, Pedro Delicado e Jorge Mateu. Continuous timevarying kriging for spatial prediction of functional data: An environmental application. Journal of agricultural, biological, and environmental statistics, 15(1):66-82. Citado na pág. 2, 3, 27, 28, $35,45,56,63,64$

Henao et al.(2011) Ramon Giraldo Henao, Pedro Delicado e Jorge Mateu. Ordinary kriging for function-valued spatial data. Environmental and Ecological Statistics, 18(3):411-426. Citado na pág. $2,3,22,25,45,63,64$

Hengl et al.(2007) Tomislav Hengl, Gerard BM Heuvelink e David G Rossiter. About regressionkriging: from equations to case studies. Computers \& Geosciences, 33(10):1301-1315. Citado na pág. 2

Hevesi et al.(1992) Joseph A. Hevesi, Jonathan D. Istok e Alan L. Flint. Precipitation estimation in mountainous terrain using multivariate geostatistics. part i: Structural analysis. Journal of Applied Meteorology, 31(7):661-676. Citado na pág. 1

Horváth e Kokoszka(2012) Lajos Horváth e Piotr Kokoszka. Inference for functional data with applications, volume 200. Springer Science \& Business Media. Citado na pág. 2, 5

Ignaccolo et al.(2014) Rosaria Ignaccolo, Jorge Mateu e Ramon Giraldo. Kriging with external drift for functional data for air quality monitoring. Stochastic Environmental Research and Risk Assessment, 28(5):1171-1186. Citado na pág. 2

Jerrett et al.(2005) Michael Jerrett, Altaf Arain, Pavlos Kanaroglou, Bernardo Beckerman, Dimitri Potoglou, Talar Sahsuvaroglu, Jason Morrison e Chris Giovis. A review and evaluation of intraurban air pollution exposure models. Journal of Exposure Science and Environmental Epidemiology, 15(2):185-204. Citado na pág. 1

John W. Eaton e Wehbring(2015) Sören Hauberg John W. Eaton, David Bateman e Rik Wehbring. GNU Octave version 4.0.0 manual: a high-level interactive language for numerical computations. URL http://www.gnu.org/software/octave/doc/interpreter. Citado na pág. 65

Journel e Huijbregts(1978) A. G. Journel e Ch. J. Huijbregts. Mining Geostatistics. Academic Press, New York. Citado na pág. 1 
Krige(1951) Danie Gerhardus Krige. A statistical aproach to some mine valuation and allied problems in the witwartersland. Dissertação de Mestrado, University of the Witwatersland, Johannesburg. Citado na pág. 2

Mallat(1989a) Stephane G Mallat. Multiresolution approximations and wavelet orthonormal bases of $L^{2}(\mathbb{R})$. Transactions of the American Mathematical Society, 315(1):69-87. Citado na pág. 18

Mallat(1989b) Stephane G Mallat. A theory for multiresolution signal decomposition: the wavelet representation. IEEE Transactions on Pattern Analysis and Machine Intelligence, 11(7):674693. Citado na pág. 18

Matheron(1963) Georges Matheron. Principles of geostatistics. Economic geology, 58(8):12461266. Citado na pág. 2

Meyer e Ryan(1993) Y Meyer e RD Ryan. Wavelets: Algorithms and applications. SIAM, Philadelphia, PA. Citado na pág. 12

Meyer(1985) Yves Meyer. Principe d'incertitude, bases hilbertiennes et algebres d'operateurs. Séminaire Bourbaki, 28:209-223. Citado na pág. 13

Meyer e Salinger(1995) Yves Meyer e David H Salinger. Wavelets and operators, volume 1. Cambridge University Press. Citado na pág. 13

Minnitt e Assibey-Bonsu(2014) R.C.A. Minnitt e W. Assibey-Bonsu. Professor Danie Gerhardus Krige. Journal of the Southern African Institute of Mining and Metallurgy, 114:vii-xi. Citado na pág. 1

Morettin(2014) Pedro Alberto Morettin. Ondas e Ondaletas: Da Análise de Fourier à Análise de Ondaletas de Séries Temporais. Edusp - Editora da Universidade de São Paulo, São Paulo. Citado na pág. 13,16

Moyeed e Papritz(2002) Rana A Moyeed e Andreas Papritz. An empirical comparison of kriging methods for nonlinear spatial point prediction. Mathematical Geology, 34(4):365-386. Citado na pág. 64

Nerini et al.(2010) David Nerini, Pascal Monestiez e Claude Manté. Cokriging for spatial functional data. Journal of Multivariate Analysis, 101(2):409-418. Citado na pág. 2, 64

Ogden(1997) Todd Ogden. Essential wavelets for statistical applications and data analysis. Springer. Citado na pág. 25, 93

Omre e Halvorsen(1989) Henning Omre e Kjetil B Halvorsen. The bayesian bridge between simple and universal kriging. Mathematical Geology, 21(7):767-786. Citado na pág. 63 
Ostro et al.(1999) Bart D Ostro, Susan Hurley e Michael J Lipsett. Air pollution and daily mortality in the coachella valley, california: a study of pm10 dominated by coarse particles. Environmental research, 81(3):231-238. Citado na pág. 59

Pilz e Spöck(2008) Jürgen Pilz e Gunter Spöck. Why do we need and how should we implement bayesian kriging methods. Stochastic Environmental Research and Risk Assessment, 22(5): 621-632. Citado na pág. 63

Pope III e Dockery(2006) C Arden Pope III e Douglas W Dockery. Health effects of fine particulate air pollution: lines that connect. Journal of the air \& waste management association, 56 (6):709-742. Citado na pág. 59

Pope III et al.(1991) C Arden Pope III, Douglas W Dockery, John D Spengler e Mark E Raizenne. Respiratory health and pm10 pollution: a daily time series analysis. American Review of Respiratory Disease, 144(3_pt_1):668-674. Citado na pág. 59

R Core Team(2015) R Core Team. R: A Language and Environment for Statistical Computing. R Foundation for Statistical Computing, Vienna, Austria, 2015. URL https://www.R-project.org/. Citado na pág. 65

Ramsay e Dalzell(1991) J. O. Ramsay e C. J. Dalzell. Some tools for functional data analysis. Journal of the Royal Statistical Society. Series B (Methodological), 53(3):539-572. Citado na pág. 2

Ramsay(2006) James O Ramsay. Functional data analysis. Wiley Online Library. Citado na pág. 2, 5,56

Ramsay e Silverman(2002) James O Ramsay e Bernard W Silverman. Applied functional data analysis: methods and case studies, volume 77. Springer New York. Citado na pág. 2, 5, 6

Ramsay et al.(2009) James O. Ramsay, Giles Hooker e Spencer Graves. Functional Data Analysis with $R$ and MATLAB. Springer. Citado na pág. 2, 5

Reyes et al.(2015) Adriana Reyes, Ramón Giraldo e Jorge Mateu. Residual kriging for functional spatial prediction of salinity curves. Communications in Statistics-Theory and Methods, 44(4): 798-809. Citado na pág. 2

Salazar et al.(2015) Elías Salazar, Ramón Giraldo e Emilio Porcu. Spatial prediction for infinitedimensional compositional data. Stochastic Environmental Research and Risk Assessment, 29 (7). Citado na pág. 2

Schwartz et al.(1993) Joel Schwartz, Daniel Slater, Timothy V Larson, William E Pierson e Jane Q Koenig. Particulate air pollution and hospital emergency room visits for asthma in seattle. American Review of Respiratory Disease, 147(4):826-831. Citado na pág. 59

Seaton et al.(1995) Anthony Seaton, D Godden, W MacNee e K Donaldson. Particulate air pollution and acute health effects. The Lancet, 345(8943):176-178. Citado na pág. 59 
Stein e Corsten(1991) A Stein e LCA Corsten. Universal kriging and cokriging as a regression procedure. Biometrics, 47(2):575-587. Citado na pág. 3

Stein(2012) Michael L Stein. Interpolation of spatial data: some theory for kriging. Springer Science \& Business Media. Citado na pág. 7

Styan(1973) George PH Styan. Hadamard products and multivariate statistical analysis, volume 6. Elsevier. Citado na pág. 38, 39

Sun et al.(2012) Ying Sun, Bo Li e Marc G Genton. Geostatistics for large datasets. Em Advances and challenges in space-time modelling of natural events, páginas 55-77. Springer. Citado na pág. 66

Vidakovic(2009) Brani Vidakovic. Statistical modeling by wavelets, volume 503. John Wiley \& Sons. Citado na pág. 25

Wackernagel(2003) Hans Wackernagel. Multivariate geostatistics. Springer. Citado na pág. 35 